\title{
Theory of neutrinoless double beta decay
}

\author{
J D Vergados ${ }^{1,2}, \mathbf{H}$ Ejiri $^{3,4}$ and F Šimkovic ${ }^{5,6}$ \\ ${ }^{1}$ Theoretical Physics Division, University of Ioannina, GR-451 10, Ioannina, Greece. \\ ${ }^{2}$ CERN, Theory Division, Geneva, Switzerland. \\ E-mail: vergados@uoi.gr \\ ${ }^{3}$ RCNP, Osaka University, Osaka, 567-0047, Japan. \\ ${ }^{4}$ Nuclear Science, Czech Technical University, Brehova, Prague, Czech Republic. \\ E-mail: ejiri@rcnp.osaka-u.ac.jp \\ ${ }^{5}$ Laboratory of Theoretical Physics, JINR, 141980 Dubna, Moscow region, Russia. \\ ${ }^{6}$ Department of Nuclear Physics and Biophysics, Comenius University, Mlynská \\ dolina F1, SK-842 15 Bratislava, Slovakia.
}

E-mail: Fedor.Simkovic@fmph.uniba.sk

\begin{abstract}
Neutrinoless double beta decay, which is a very old and yet elusive process, is reviewed. Its observation will signal that lepton number is not conserved and the neutrinos are Majorana particles. More importantly it is our best hope for determining the absolute neutrino mass scale at the level of a few tens of meV. To achieve the last goal certain hurdles have to be overcome involving particle, nuclear and experimental physics.

Nuclear physics is important for extracting the useful information from the data. One must accurately evaluate the relevant nuclear matrix elements, a formidable task. To this end, we review the sophisticated nuclear structure approaches recently been developed, which give confidence that the needed nuclear matrix elements can be reliably calculated employing different methods: a) the various versions of the Quasiparticle Random Phase Approximations, b) the interacting boson model, c) the energy density functional method and d) the large basis Interacting Shell Model. It is encouraging that, for the light neutrino mass term at least, these vastly different approaches now give comparable results.

From an experimental point of view it is challenging, since the life times are long and one has to fight against formidable backgrounds. One needs large isotopically enriched sources and detectors with high energy resolution, low thresholds and very low background.

If a signal is found, it will be a tremendous accomplishment. Then, of course, the real task is going to be the extraction of the neutrino mass from the observations. This is not trivial, since current particle models predict the presence of many mechanisms other than the neutrino mass, which may contribute or even dominate this process. We will, in particular, consider the following processes:
\end{abstract}

(i) The neutrino induced, but neutrino mass independent contribution.

(ii)Heavy left and/or right handed neutrino mass contributions.

(iii)Intermediate scalars (doubly charged etc).

(iv)Supersymmetric (SUSY) contributions.

We will show that it is possible to disentangle the various mechanisms and unambiguously extract the important neutrino mass scale, if all the signatures of the reaction are searched in a sufficient number of nuclear isotopes. 
PACS numbers: 14.60.Pq 13.15.+g 23.40.Bw 29.40.-n 29.40.Cs

Submitted to: Reports on Progress in Physics 


\section{Contents}

1 A brief history of double beta decay 5

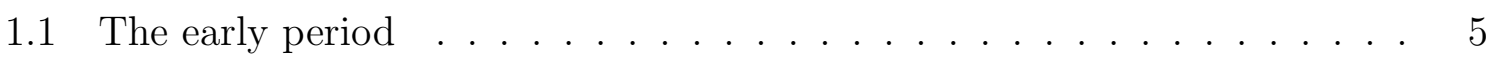

1.2 The period of scepticism . . . . . . . . . . . . . . . 6

1.3 The period of Grand Unified Theories . . . . . . . . . . . . . . . . 7

1.4 The period of massive neutrinos - the current period . . . . . . . . . 8

1.5 The period of Majorana neutrinos? . . . . . . . . . . . . . . . . . . 9

2 An overview

3 The neutrino mass matrix $\quad \underline{16}$

3.1 Neutrino masses at tree level . . . . . . . . . . . . . . . . . . . 16

3.2 Neutrino masses at the loop level . . . . . . . . . . . . . . . . . 19

3.3 SUSY, GUT's and Family symmetries . . . . . . . . . . . . . . . . . . . . . . . . . . . . .

3.4 Neutrino mixing . . . . . . . . . . . . . . . . . . 20

4 The absolute scale of the neutrino mass

5 The Majorana neutrino mechanism 29

5.1 The Majorana neutrino mass mechanism . . . . . . . . . . . . . . . 30

5.2 The neutrino mass independent mechanism (leptonic left-right interference, $\lambda$ and $\eta$ terms). . . . . . . . . . . . . . . . . . . . 32

5.3 Another neutrino mass independent mechanism (majoron emission) . . . 35

6 Mechanisms without intermediate neutrinos $\quad 36$

6.1 The direct decay of doubly charged particles to leptons . . . . . . . . . . 36

6.2 The R-parity violating contribution to $0 \nu \beta \beta$ decay. . . . . . . . . . . . 37

6.2.1 The contribution arising from the bilinears in the superpotential. 38

6.2.2 The contribution arising from the cubic terms in the superpotential. 38

6.2.3 The lepton number violating parameters from the cubic terms without intermediate neutrinos. . . . . . . . . . . . . 39

6.2.4 The case of light intermediate neutrinos . . . . . . . . . 40

7 Handling the short range transition operators 42

7.1 The mode involving only nucleons . . . . . . . . . . . . . . . . . 43

7.2 The pion mode in R-parity induced $0 \nu \beta \beta$ decay. . . . . . . . . . . . . 43

8 Experimental aspects of double beta decays

8.1 Progress of DBD experiments . . . . . . . . . . . . . 47

8.1.1 Experimental aspects of neutrinoless double beta decays . . . . 47

8.1.2 Progresses of DBD experiments. . . . . . . . . . . . . . 48

8.2 Methods and detectors for DBD experiments . . . . . . . . . . . . 49

8.2.1 Methods for DBD experiments. . . . . . . . . . . . 49 


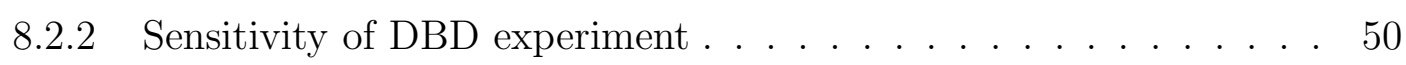

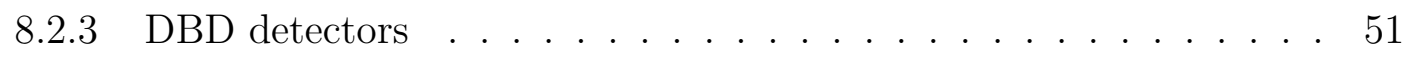

8.3 Present status and future projects of DBD experiments . . . . . . . . 52

8.3.1 Neutrinoless double beta decays . . . . . . . . . . . . . . . . 52

8.3.2 Two neutrino double beta decays . . . . . . . . . . . . . 54

8.3.3 High sensitivity experiments ................ 54

8.4 Experimental studies of DBD matrix elements . . . . . . . . . . . 58

8.4.1 Experimental probes for DBD matrix elements . . . . . . . . 58

8.4.2 DBD matrix elements via low lying intermediate states . . . . . 62

8.5 Two-neutrino double beta decay and bosonic neutrino . . . . . . . . . 65

9 Effective transition operators 65

9.1 Transition operators resulting from light neutrino exchange . . . . . . . 67

9.2 Transition operators resulting the heavy neutrino exchange mechanism . 68

9.3 Transition operators resulting from the R-parity breaking SUSY mechanism 68

9.4 Transition operators resulting from the squark-neutrino mechanism . . . 69

10 Nuclear matrix elements $\quad \mathbf{7 0}$

10.1 Uncertainties in calculated NMEs . . . . . . . . . . . . . . . . . . . . . . . . . . . . . . . .

10.2 Anatomy of NMEs . . . . . . . . . . . . . . . . . . 79

11 Distinguishing the various mechanisms $\quad 80$

11.1 Dominance of a single mechanism . . . . . . . . . . . . . . . . . . . . . . . . . . .

11.2 Nuclear matrix elements of exotic mechanisms . . . . . . . . . . . . . 83

11.3 Two or more competing mechanisms . . . . . . . . . . . . 87

12 Resonant neutrinoless double electron capture $\quad 90$

13 Concluding remarks $\quad 94$ 


\section{A brief history of double beta decay}

A brief history of the double-beta decay is presented.

\subsection{The early period}

Double beta decay (DBD), namely the two-neutrino double-beta decay ( $2 \nu \beta \beta$-decay)

$$
(A, Z) \rightarrow(A, Z+2)+e^{-}+e^{-}+\bar{\nu}_{e}+\bar{\nu}_{e},
$$

was first considered in publication [1] of Maria Goeppert-Mayer in 1935. It was Eugene Wigner, who suggested this problem to the author of [1] about one year after the Fermi weak interaction theory appeared. In the work of Maria Goeppert-Mayer [1] an expression for the $2 \nu \beta \beta$-decay rate was derived and a half-life of $10^{17}$ years was estimated by assuming a Q-value of about $10 \mathrm{MeV}$.

Two years later (1937) Ettore Majorana formulated a new theory of neutrinos, whereby the neutrino $\nu$ and the antineutrino $\bar{\nu}$ are indistinguishable, and suggested antineutrino induced $\beta^{-}$-decay for experimental verification of this hypothesis [2]. Giulio Racah was the first, who proposed testing Majorana's theory with real neutrinos by chain of reactions

$$
(A, Z) \rightarrow(A, Z+1)+e^{-}+\nu, \quad \nu+\left(A^{\prime}, Z^{\prime}\right) \rightarrow\left(A^{\prime}, Z^{\prime}+1\right)+e^{-},
$$

which is allowed in the case of the Majorana neutrino and forbidden in the case of Dirac neutrino [3. In 1939, Wolfgang Furry considered for the first time neutrinoless double beta decay $(0 \nu \beta \beta$-decay),

$$
(A, Z) \rightarrow(A, Z+2)+e^{-}+e^{-},
$$

a Racah chain of reactions with virtual neutrinos $\left((A, Z+1) \equiv\left(A^{\prime}, Z^{\prime}\right)\right)$ [4]. Here $A, A^{\prime}$ are the nuclear mass numbers and $Z, Z^{\prime}$ the charges of the nuclei involved. The available energy $\Delta$ is equal to the $Q$-value of the reaction, i.e. the mass difference of the ground states of the two atoms involved.

In 1952 Henry Primakoff [5] calculated the electron-electron angular correlations and electron energy spectra for both the $2 \nu \beta \beta$-decay and the $0 \nu \beta \beta$-decay, producing a useful tool for distinguishing between the two processes.

At that time nothing was known about the chirality suppression of the $0 \nu \beta \beta$ decay. It was believed that, due to a considerable phase-space advantage, the $0 \nu \beta \beta$ decay mode dominates the double beta decay rate. Starting 1950 this phenomenon was exploited in early geochemical, radiochemical and counter experimentst. It was found that the measured lower limit on the $\beta \beta$-decay half-life far exceeds the values expected for this process, $T_{1 / 2}^{0 \nu} \sim 10^{12}-10^{15}$ years. In 1955 the Raymond Davis experiment [7], which searched for the antineutrinos from reactor via nuclear reaction $\bar{\nu}_{e}+{ }^{37} \mathrm{Cl} \rightarrow{ }^{37} \mathrm{Ar}+e^{-}$, produced a zero result. The above experiments were interpreted

$\ddagger$ For more detailed historical review of the experimental activities in the field of double-beta decay we recommend reader a recent review on this subject by A. Barabash [6]. 
as proof that the neutrino was not a Majorana particle, but a Dirac particle. This prompted the introduction of the lepton number to distinguish the neutrino from its antiparticle. The assumption of lepton number conservation allows the $2 \nu \beta \beta$-decay but forbids the $0 \nu \beta \beta$-decay, in which lepton number is changed by two units.

In 1949 Fireman reported the first observation of the $\beta \beta$-decay of ${ }^{124} \mathrm{Sn}$ in a laboratory experiment [8], but he disclaimed it later [9]. The first geochemical observation of the $\beta \beta$-decay, with an estimated half-life $T_{1 / 2}\left({ }^{130} \mathrm{Te}\right)=1.4 \times 10^{21}$ years, was announced by Ingram and Reynolds in 1950 [10]. Extensive studies have been made by Gentner and Kirsten [11, 12] and others [13, 14] on such rare-gass isotopes as ${ }^{82} \mathrm{Kr}$, ${ }^{128} \mathrm{Xe}$, and ${ }^{130} \mathrm{Xe}$, which are $\beta \beta$-decay products of ${ }^{82} \mathrm{Se}{ }^{128} \mathrm{Te}$, and ${ }^{130} \mathrm{Te}$, respectively, obtaining half lives around $10^{21} \mathrm{y}$ for ${ }^{130} \mathrm{Te}$.

\subsection{The period of scepticism}

Shortly after its theoretical formulation by Lee and Yang, parity violation in the weak interaction was established by two epochal experiments. In $1957 \mathrm{Wu}$ et al. discovered the asymmetry in the angular distribution of the $\beta$-particles emitted relative to the spin orientation of the parent nucleus ${ }^{60}$ Co. A year later Goldhaber et al [15] discovered that the neutrinos are polarized and left handed by measuring the polarization of a photon, moving back to back with the neutrino, produced by the de-excitation of a ${ }^{152} E u^{*}$ nucleus after K-capture. In 1958 the seemingly confused situation was simplified in the form of the vector-axial vector ( $\mathrm{V}-\mathrm{A}$ ) theory of weak interactions describing maximal parity violation in agreement with available data. In order to account for the chiral symmetry breaking of the weak interaction only left handed fermions participate and the mediating particles must be vectors of spin 1, which are left handed in the sense that they couple only to left handed fermions.

The maximal parity violation is easily realized in the lepton sector by using the two-component theory of a massless neutrino, proposed in 1957 by L. Landau, T.D. Lee, C.N. Yang and A. Salam (This idea was first developed by H. Weyl in 1929, but it was rejected by Pauli in 1933 on the grounds that it violates parity.). In this theory, neutrinos are left handed and antineutrinos are right-handed, leading automatically to the $\mathrm{V}$ - A couplings.

With the discovery of parity violation, it became apparent that the Majorana/Dirac character of the electron neutrino was still in question. The particles that participate in the $0 \nu \beta \beta$-decay reaction at nucleon level are right-handed antineutrino $\bar{\nu}_{e}$ and left handed neutrino $\nu_{e}$ :

$$
n \rightarrow p+e^{-}+\bar{\nu}_{e}^{R}, \quad \nu_{e}^{L}+n \rightarrow p+e^{-} .
$$

Thus even if the neutrino is a (massless) Majorana particle, since the first neutrino has the wrong helicity for absorption by a neutron, the absence of the $0 \nu \beta \beta$-decay implies neither a Dirac electron neutrino nor a conserved lepton number.

The requirement that both lepton number conservation and the $\gamma_{5}$ invariance of the weak current had to be violated, in order the $0 \nu \beta \beta$-decay to occur, discouraged 
experimental searches.

\subsection{The period of Grand Unified Theories}

The maximal violation of parity (and of charge-conjugation) symmetry is accommodated in the Standard Model (SM), which describes jointly weak and electromagnetic interactions. This model was developed largely upon the empirical observations of nuclear beta decay during the latter half of the past century. Despite the phenomenological success of the SM, the fundamental origin of parity violation has not been understood. In spite of the fact that the SM represents the simplest and the most economical theory, it has not been considered as the ultimate theory of nature. It was assumed that, most likely, it describes a low energy approximation to a more fundamental theory.

With the development of modern gauge theories during the last quarter of the previous century, perceptions began to change. In the SM it became apparent that the assumption of lepton number conservation led to the neutrino being strictly massless, thus preserving the $\gamma_{5}$-invariance of the weak current. With the development of Grand Unified Theories (GUT's) of the electroweak and strong interactions, the prejudice has grown that lepton number conservation was the result of a global symmetry not of a gauge symmetry and had to be broken at some level. In other words modern GUT's and supersymmetric (SUSY) extensions of the SM suppose that such conservation laws of the SM may be violated to some small degree. The lepton number may only appear to be conserved at low energies because of the large grand unified mass scale $\Lambda_{G U T}$ governing its breaking. Within the proposed see-saw mechanism one expects the neutrino to acquire a small Majorana mass of a size $\sim(\text { light mass })^{2} / \Lambda_{G U T}$, where "light mass" is typically that of a quark or charged lepton. The considerations of a sensitivity of the $0 \nu \beta \beta$-decay experiments to a neutrino mass $m_{\nu} \sim 1 \mathrm{eV}$ became the genesis of a new interest to double beta decay. Thus the interest in $0 \nu \beta \beta$-decay was resurrected through the pioneering work of Kotani and his group [16], which brought it again to the attention of the nuclear physics community.

Neutrino masses require either the existence of right-handed neutrinos or require violation of the lepton number $(\mathrm{LN})$ so that Majorana masses are possible. So, one is forced to go beyond the minimal models again, whereby LF and/or LN violation can be allowed in the theory. A good candidate for such a theory is the left-right symmetric model of Grand Unification inaugurated by Salam, Pati, Mohapatra and Senjanović [17, 18, 19] and especially models based on $\mathrm{SO}(10)$, which have first been proposed by Fritzsch and Minkowski [20], with their supersymmetric versions [21, 22, 23]. The leftright symmetric models, representing generalization of the $S U(2)_{L} \otimes U(1) \mathrm{SM}$, predict not only that the neutrino is a Majorana particle, that means it is up to a phase identical with its antiparticle, but automatically predict the neutrino has a mass and a weak right-handed interaction.

In the left-right symmetric models the LN conservation is broken by the presence of 
the Majorana neutrino mass. The LN violation is also inherent in those SUSY theories whereby R-parity, defined as $R_{p}=(-1)^{3 B+L+2 S}$, with $S, B$, and $L$ being the spin, baryon and lepton number, respectively is not a conserved quantity anymore.

The $0 \nu \beta \beta$-decay, which involves the emission of two electrons and no neutrinos, has been found as a powerful tool to study the LN conservation. Schechter and Valle proved that, if the $0 \nu \beta \beta$-decay takes place, regardless of the mechanism causing it, the neutrinos are Majorana particles with non-zero mass [24, 25]. It was recognized that the GUT's and R-parity violating SUSY models offer a plethora of the $0 \nu \beta \beta$-decay mechanisms triggered by exchange of neutrinos, neutralinos, gluinos, leptoquarks, etc. [26, 27, 28].

The experimental effort concentrated on high $Q_{\beta \beta}$ isotopes, in particular on ${ }^{48} \mathrm{Ca}$, ${ }^{76} \mathrm{Ge},{ }^{82} \mathrm{Se},{ }^{96} \mathrm{Zr},{ }^{100} \mathrm{Mo},{ }^{116} \mathrm{Cd},{ }^{130} \mathrm{Te},{ }^{136} \mathrm{Xe}$ and ${ }^{150} \mathrm{Nd}$ [29, 30, 31]. In 1987 the first actual laboratory observation of the two neutrino double beta decay ( $2 \nu \beta \beta$-decay) was done for ${ }^{82}$ Se by M. Moe and collaborators [32], who used a time projection chamber. Within the next few years, experiments employing counters were able to detect $2 \nu \beta \beta$-decay of many nuclei. In addition, the experiments searching for the signal of the $0 \nu \beta \beta$-decay pushed by many orders of magnitude the experimental lower limits for the $0 \nu \beta \beta$-decay half-life of different nuclei.

\subsection{The period of massive neutrinos - the current period}

Various early measurements of neutrinos produced in the sun, in the atmosphere, and by accelerators suggested that neutrinos might oscillate from one "flavor" (electron, muon, and tau) to another, expected as a consequence of non-zero neutrino mass. Non-zero neutrino mass can be accommodated by fairly straightforward extensions of the SM of particle physics. Starting 1998 we have a convincing evidence about the existence of neutrino masses due to SuperKamiokande [33], SNO, [34] KamLAND [35] and other experiments.

Thus neutrino oscillations have supplied additional information in constructing Grand Unified Theories of physics. It also has provided additional input for cosmologists and opened new perspectives for observation of the $0 \nu \beta \beta$-decay.

So far the $2 \nu \beta \beta$-decay has been recorded for eleven nuclei $\left({ }^{48} \mathrm{Ca},{ }^{76} \mathrm{Ge},{ }^{82} \mathrm{Se}\right.$, ${ }^{96} \mathrm{Zr},{ }^{100} \mathrm{Mo},{ }^{116} \mathrm{Cd},{ }^{128} \mathrm{Te},{ }^{130} \mathrm{Te},{ }^{150} \mathrm{Nd},{ }^{136} \mathrm{Xe},{ }^{238} \mathrm{U}$ ) [29, 30, 31]. In addition, the $2 \nu \beta \beta$-decay of ${ }^{100} \mathrm{Mo}$ and ${ }^{150} \mathrm{Nd}$ to $0^{+}$excited state of the daughter nucleus has been observed and the two-neutrino double electron capture process in ${ }^{130} \mathrm{Ba}$ has been recorded. Experiments studying $2 \nu \beta \beta$-decay are presently approaching a qualitatively new level, when high-precision measurements are performed not only for half-lives but also for all other observables of the process. As a result, a trend is emerging towards thorough investigation of all aspects of $2 \nu \beta \beta$-decay, and this will furnish very important information about the values of nuclear matrix elements, the parameters of various theoretical models, and so on. In this connection, one may expect advances in the calculation of nuclear matrix elements and in the understanding of the nuclear-physics 
aspects of double beta decay.

Neutrinoless double beta decay has not yet been confirmed. The strongest limits on the half-life of the $0 \nu \beta \beta$-decay were set in Heidelberg-Moscow [36], NEMO3 [37, 38], CUORICINO [39] and KamLAND-Zen [40] experiments:

$$
\begin{aligned}
& T_{1 / 2}^{0 \nu}\left({ }^{76} \mathrm{Ge}\right) \geq 1.9 \times 10^{25} \mathrm{y}, \quad T_{1 / 2}^{0 \nu}\left({ }^{100} \mathrm{Mo}\right) \geq 1.0 \times 10^{24} \mathrm{y}, \\
& T_{1 / 2}^{0 \nu}\left({ }^{130} \mathrm{Te}\right) \geq 3.0 \times 10^{24} \mathrm{y}, \quad T_{1 / 2}^{0 \nu}\left({ }^{136} \mathrm{Xe}\right) \geq 5.7 \times 10^{24} \mathrm{y} .
\end{aligned}
$$

There is also a claim for an observation of the $0 \nu \beta \beta$-decay of ${ }^{76} \mathrm{Ge}$ with half-life $T_{1 / 2}^{0 \nu}=2.23_{-0.31}^{+0.44} \times 10^{25}$ years [41, 42]. One of the the goals of the upcoming GERDA experiment [43] is to put this claim to a test by improving the sensitivity limit of the detection by more than an order of magnitude. The next generation experiments, which will be using several other candidate nuclei, will eventually be able to achieve this goal as well [31.

\subsection{The period of Majorana neutrinos?}

There is a hope that the period of Majorana neutrinos is not far. This period should start by a direct and undoubtable observation of the $0 \nu \beta \beta$-decay. It would establish that neutrinos are Majorana particles, and a measurement of the decay rate, when combined with neutrino oscillation data and a reliable calculation of nuclear matrix elements, would yield insight into all three neutrino mass eigenstates.

\section{An overview}

The question of neutrino masses and mixing is one of the most important issues of modern particle physics. It has already been discussed in a number of excellent reviews [44, 45, 46, 47, 48] and its relevance to the $0 \nu \beta \beta$-decay will also be briefly discussed in this report.

Today, seventy five years later, $0 \nu \beta \beta$-decay (3), continues to be one of the most interesting processes. The experimental status and prospects regarding this process will be reviewed in section 8 . The corresponding non exotic $2 \nu \beta \beta$ (1) has been observed in many systems, see section 8 ,

If the neutrinos are Majorana particles other related processes in which the charge of the nucleus is decreased by two units may also occur, if they happen to be allowed by energy and angular momentum conservation laws, e.g.

$$
(A, Z) \rightarrow(A, Z-2)+e^{+}+e^{+} \quad(0 \nu \text { positron emission }) .
$$

Here the available energy is $\Delta=Q-4 m_{e} c^{2}$, i.e., everything else being equal, it is somewhat kinematically disfavored compared to the usual two electron emission for which the available energy $\Delta$ is equal to the $Q$ value.

Electron positron conversion:

$$
(A, Z)+e_{b}^{-} \rightarrow(A, Z-2)+e^{+} \quad(0 \nu \text { electron positron conversion }) .
$$




\section{Nuclear Femto Laboratory \\ for $\beta \beta$ experiments}
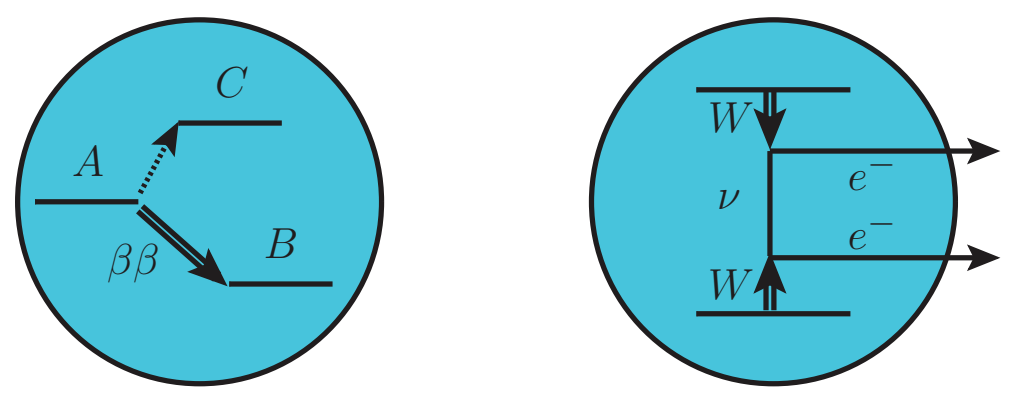

Figure 1: Schematic diagrams of $\beta \beta$ decays in nuclear femto $\left(10^{-15} \mathrm{~m}\right)$ laboratories, where single $\beta$-decay is not allowed and neutrinoless DBD is much enhanced. Left hand side: DBD decay scheme. Right hand side: Neutrinoless DBD with the Majorana $\nu$ exchange between two nucleons [49].

$\Delta=Q-2 m_{e} c^{2}-\varepsilon_{b}$, where $\varepsilon_{b}$ is a binding energy of the absorbed atomic electron.

The resonant neutrinoless double electron capture ( $0 \nu$ ECEC),

$$
\left.(A, Z)+e_{b}^{-}+e_{b}^{-} \rightarrow(A, Z-2)^{* *}, \text { (resonant } 0 \nu \text { double electron capture }\right),(8)
$$

was first considered very long time ago by Winter [50]. It is always allowed, whenever (77) is. This reaction was expounded in more detail later [51, 52] as a two step process: In the first step the two neutral atoms, (A,Z) and the excited (A,Z-2), get admixed via the lepton number violating interaction. In the second step the (A,Z-2) atom and, possibly, the nucleus de-excite. The available energy is $\Delta=Q-B_{2 h}, B_{2 h}$ being the energy of two electron holes in the atomic shells of daughter nucleus.

Decays to excited states are in some cases possible. Then in addition to x rays one has various decay modes with emission of a single $\gamma$, a pair of $\gamma$ 's, internal electron-positron pair formation and emission of electron by internal conversion [53], i.e.

$$
(A, Z)+e_{b}^{-}+e_{b}^{-} \rightarrow(A, Z-2)+X, \quad X=\gamma, 2 \gamma, e^{+} e^{-}, e_{i n t}^{-}
$$

The life time expected was very long, since the above mixing amplitude was tiny compared to the energy difference of the two atoms involved. It has recently, however, been gaining in importance [54, 55] after ion Penning traps [56] made it possible to accurately determine the $Q$ values, which gave rise to the the presence of resonances. This, in turn, could lead to an increase of the width by many orders of magnitude, see section 12 for details.

Another lepton number violating process, not hindered by energy conservation in 
any nuclear system, involves the neutrinoless bound muon capture [57, 58,

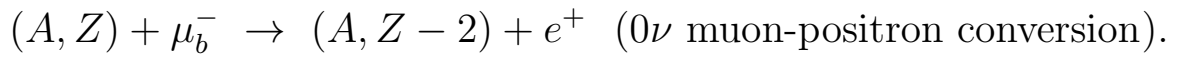

The best experimental limit on the muon to positron conversion branching ratio has been established at PSI [59] for the ${ }^{48} \mathrm{Ti}$ nuclear target. The muonic analogue of neutrinoless double beta decay [60, 61],

$$
(A, Z)+\mu_{b}^{-} \rightarrow(A, Z-2)+\mu^{+} \quad(0 \nu \text { muon-muon conversion })
$$

has never been searched for experimentally. For bound muon in atom energy conservation of the process is very restrictive. It is satisfied only for three isobars, namely ${ }^{44} \mathrm{Ti},{ }^{72} \mathrm{Se}$ and ${ }^{82} \mathrm{Sr}$.

The above processes are expected to occur whenever one has lepton number violating interactions. Lepton number, being a global quantity, is not sacred, but it is expected to be broken at some level. In short, these processes pop up almost everywhere, in every theory. On the other hand since, if there exist lepton number violating interactions, the neutrinos have to be Majorana particles, all the above processes can, in principle, decide whether or not the neutrino is a Majorana particle, i.e. it coincides with its own antiparticle. This is true even if these processes are induced not by intermediate neutrinos but by other mechanisms as we will see below.

Neutrinoless double beta decay (Eq. (3) ) seems to be the most likely to yield the information [44, 62, 63, 64, 65, 66, 67, 68] we are after. For this reason we will focus our discussion on this reaction, but we will pay some attention to resonant neutrinoless double electron capture, which has recently been revived [51, 52, 54, 55, 69, 70, 71], since its observation seems to be a realistic possibility [54, 55]. We will only peripherally discuss the other less interesting processes [51.

From a nuclear physics [65, 66, 72, 73, 74, 75, 76] point of view, calculating the relevant nuclear matrix elements is indeed a challenge. First almost all nuclei, which can undergo double beta decay, are far from closed shells and some of them are even deformed. One thus faces a formidable task. Second the nuclear matrix elements are small compared to a canonical value, like the one associated with the matrix element to the (energy non allowed) double Gamow-Teller resonance or a small fraction of some appropriate sum rule. Thus, effects which are normally negligible, become important here. Third in many models the dominant mechanism for $0 \nu \beta \beta$-decay does not involve intermediate light neutrinos, but very heavy particles. Thus one must be able to cope with the short distance behavior of the relevant operators and wave functions (see section 10 for details).

From the experimental point of view it is also very challenging to measure the slowest perhaps process accessible to observation. Especially, if one realizes that even, if one obtains only lower bounds on the life time for this $0 \nu \beta \beta$-decay, the extracted limits on the theoretical model parameters may be comparable, if not better, and complementary to those extracted from the most ambitious accelerator experiments.

The recent discovery of neutrino oscillations [77, 78, 79, 80] have given the first evidence of physics beyond the Standard Model (SM) and in particular they indicate 
that the neutrinos are massive particles. They were able to show that the neutrinos are admixed, determined two of the mixing angles and set a stringent limit on the third (for a global analysis see, e.g., [81]). Furthermore they determined one square mass difference and the absolute value of the other. Neutrino oscillations, however, cannot determine:

- Whether the neutrinos are Majorana or Dirac particles.

It is obviously important to proceed further and decide on this important issue. Neutrinoless double beta decay can achieve this, even if, as we have mentioned, there might be processes that dominate over the conventional intermediate neutrino mechanism of $0 \nu \beta \beta$-decay. It has been known that whatever the lepton number violating process is, which gives rise to $0 \nu \beta \beta$-decay, it can be used to generate a Majorana mass for the neutrino [24]. This mechanism, however, may not be the dominant mechanism for generating the neutrino mass [82].

- The scale of the neutrino masses.

These experiments can measure only mass squared differences.

This task can be accomplished by astrophysical observations or via other experiments involving low energy weak decays, like triton decay or electron capture, or the $0 \nu \beta \beta$-decay. It seems that for a neutrino mass in $\mathrm{meV},\left(10^{-3} \mathrm{eV}\right)$, region, the best process to achieve this is the $0 \nu \beta \beta$-decay. The extraction of neutrino masses from such observations will be discussed in detail and compared with each other later (see section 4).

- The neutrino hierarchy

They cannot at present decide which scenario is realized in nature, namely the degenerate, the normal hierarchy or the inverted hierarchy. They may be able to distinguish between the two hierarchies in the future by observing the wrong sign muons in neutrino factories [83, 84].

For details on such issues see a recent review [85].

The study of the $0 \nu \beta \beta$-decay is further stimulated by the development of GUT's and supersymmetric models (SUSY) representing extensions of the $S U(2)_{L} \otimes U(1)$ standard model. The GUT's and SUSY offer a variety of mechanisms which allow the $0 \nu \beta \beta$-decay to occur [86].

The best known mechanism leading to $0 \nu \beta \beta$-decay is via the exchange of a Majorana neutrino between the two decaying neutrons [44, 62, 63, 64, 65, 66, 87]. Nuclear physics allows us to study the light $\left(m_{\nu} \ll m_{e}\right)$ and heavy $\left(m_{\nu} \gg m_{p}\right)$ neutrino components separately. In the presence of only left handed currents and for the light intermediate neutrino components, the obtained amplitude is proportional to a suitable average neutrino mass, which vanishes in the limit in which the neutrinos become Dirac particles. On the other hand in the case of heavy Majorana neutrino components the amplitude is proportional to the average of the inverse of the neutrino mass, i.e. it is again suppressed. In the presence of right handed currents one can have a contribution similar to the one above for heavy neutrinos but involving a different (larger) average inverse mass with 
some additional suppression due to the the fact the right handed gauge boson, if it exists, is heavier than the usual left handed one.

In the presence of right handed currents it is also possible to have interference between the leptonic left and right currents, $j_{L}-j_{R}$ interference. In this case the amplitude in momentum space becomes proportional to the 4-momentum of the neutrino and, as a result, only the light neutrino components become important. One now has two possibilities. First the two hadronic currents have a chirality structure of the same kind, i.e. $J_{L}-J_{R}$. Then one can extract from the data a dimensionless parameter $\lambda$, which is proportional to the square of the ratio of the masses of the $\mathrm{L}$ and $\mathrm{R}$ gauge bosons, $\kappa=\left(m_{L} / m_{R}\right)^{2}$. Second the two hadronic currents are left handed, which can happen via the mixing $\epsilon$ of the two bosons. The relevant lepton number violating parameter $\eta$ is now proportional to this mixing $\epsilon$. Both of these parameters, however, also involve the neutrino mixing. They are, in a way, proportional to the mixing between the light and heavy neutrinos.

In gauge theories one has, of course, many more possibilities. Exotic intermediate scalars may mediate $0 \nu \beta \beta$-decay 44]. These are not favored in current gauge theories and are not going to be further discussed. In superstring inspired models one may have singlet fermions in addition to the usual right handed neutrinos. Not much progress has been made on the phenomenological side of these models and they are not going to be discussed further.

In recent years supersymmetric models are taken seriously and semirealistic calculations are taking place. In standard calculations one invokes universality at the GUT's scale, employing a set of five independent parameters, and uses the renormalization group equation to obtain all parameters (couplings and particle masses) at low energies. Hence, since such parameters are in principle calculable in terms of the five input parameters, one can use experimental data to constrain the input parameters. One, then, can use the $0 \nu \beta \beta$-decay experiments to constrain the R-parity violating couplings, which cannot be specified by the theory [26, 88, 89, 90, 91, 92, 93, 94, 95]. Recent review articles [31, 65, 66, 68] also give a detailed account of some of the latest developments in this field.

From the above discussion it is clear that one has to consider the case of heavy intermediate particles. One thus has to tackle problems related to the very short ranged operators in the presence of the nuclear repulsive core. If the interacting nucleons are point-like one gets negligible contributions. We know, however that the nucleons are not point like, but that they have structure described by a quark bag with a size that can be determined experimentally. It can also be accounted for by a form factor, which can be calculated in the quark model or parametrized in a dipole shape with a parameter determined by experiment. This approach, first considered by Vergados [96], has now been adopted by almost everybody. The resulting effective operator has a range somewhat less than the inverse of the proton mass (see sect. 4 below).

Another approach in handling this problem consists of considering particles other than the nucleons present in the nuclear soup. For $0^{+} \rightarrow 0^{+}$the most important such 
particles are the pions. One thus may consider the double beta decay of pions in flight between nucleons, like

$$
\pi^{-} \longrightarrow \pi^{+}+e^{-}+e^{-} \quad, \quad n \longrightarrow p+\pi^{+}+e^{-}+e^{-} .
$$

Recognition of such contribution first appeared as a remark by the genius of Pontecorvo [97] in the famous paper in which he suggested that the ratio of the lifetimes of the ${ }^{128} \mathrm{Te}$ and ${ }^{130} \mathrm{Te}$ isotopes, which merely differ by two neutrons, is essentially independent of nuclear physics. He did not perform any estimates of such a contribution. Such estimates and calculations were first performed by Vergados [98] in the case of heavy intermediate neutrinos, .i.e. vector and axial vector currents. It was found that it yields results of the same order as the nucleon mode with the above recipe for treating the short range behavior. It was revived by the Tuebingen group [26, 27, 28, 93] in the context of R-parity violating interactions, i.e. scalar, pseudoscalar and tensor currents arising out of neutralino and gluino exchange, and it was found to dominate.

In a yet another approach one may estimate the presence of six quark clusters in the nucleus. Then, since the change of charge takes place in the same hadron there is no suppression due to the short nature of the operator, even if it is a $\delta$-function. One only needs a reliable method for estimating the probability of finding these clusters in a nucleus [99].

All the above approaches seem reasonable and lead to quite similar results. The matrix elements obtained are not severely suppressed. This gives us a great degree of confidence that the resulting matrix elements are sufficiently reliable, allowing double beta decay to probe very important physics.

The other recent development is the better description of nucleon current by including momentum dependent terms, such as the modification of the axial current due to PCAC and the inclusion of the weak magnetism terms. These contributions have been considered previously [87, 100], but only in connection with the extraction of the $\eta$ parameter mentioned above. Indeed these terms were very important in this case since they compete with the p-wave lepton wave function, which, with the usual currents, provides the lowest non vanishing contribution. Since in the mass term only s-wave lepton wave functions are relevant such terms have hitherto been neglected.

It was recently found [101], however, that for light neutrinos the inclusion of these momentum dependent terms reduces the nuclear matrix element by about $25 \%$, independently of the nuclear model employed. On the other hand for heavy neutrinos the effect can be larger and it depends on the nuclear wave functions. The reason for expecting them to be relevant is that the average momentum $\langle q\rangle$ of the exchanged neutrino is expected to be large [102. In the case of a light intermediate neutrino the mean nucleon-nucleon separation is about $2 \mathrm{fm}$ which implies that the average momentum $\langle q\rangle$ is about $100 \mathrm{MeV} / \mathrm{c}$. In the case of a heavy neutrino exchange the mean inter nucleon distance is considerably smaller and the average momentum $\langle q\rangle$ is supposed to be considerably larger.

Since $0 \nu \beta \beta$ decay is a two step process, in principle, one needs to construct and 
sum over all the intermediate nuclear states, a formidable job indeed in the case of the shell model calculations. Since, however, the average neutrino momentum is much larger compared to the nuclear excitations, one can invoke closure using some average excitation energy (this does not apply in the case of $2 \nu \beta \beta$ decays). Thus one need construct only the initial and final $0^{+}$nuclear states. This is not useful in Quasiparticle Random Phase Approximation (QRPA), since one must construct the intermediate states anyway. In any case, it was explicitly shown, taking advantage of the momentum space formalism developed by Vergados [103], that this approximation is very good [104, 105]. The same conclusion was reached independently by others [106] via a more complicated technique relying on coordinate space.

Granted that one takes into account all the above ingredients in order to obtain quantitative answers for the lepton number violating parameters from the results of $0 \nu \beta \beta$-decay experiments, it is necessary to evaluate the relevant nuclear matrix elements with high reliability. The most extensively used methods are the large basis Interacting Shell Model (ISM) calculations, (for a recent review see [65]) and QRPA( for a recent review see [66, 65]). The ISM is forced to use few single particle orbitals, while this restriction does not apply in the case of QRPA. The latter suffers, of course, from the approximations inherent in the RPA method. So a direct comparison between them is not possible.

The shell model calculations have a long history [76, 175, 107, 108, 109, 110, 111] in double beta decay calculations. In recent years it has lead to large matrix calculations in traditional as well as Monte Carlo types of formulations [72, 73, 74, 112, 113, 114]. For a more complete set of references as well as a discussion of the appropriate effective interactions see Ref. [65]).

There have been a number of QRPA calculations covering almost all nuclear targets [115, 116, 117, 118, 119, 120, 121, 122, 123, 124, 125, 126]. These involve a number of collaborations, but the most extensive and complete calculations in one way or another include the Tuebingen group. We also have seen some refinements of QRPA, like proton neutron pairing and inclusion of renormalization effects due to Pauli principle corrections [127, 128. Other less conventional approaches, like operator expansion techniques have also been employed [66].

Recently, calculations based on the Projected Hartree-Fock-Bogoliubov (PHFB) method [129, the Interacting Boson Model (IBM) [130] and the Energy Density Functional (EDF) method [131] entered the field of such calculations. The above schemes, in conjunction with the other improvements mentioned above, offer some optimism in our efforts for obtaining nuclear matrix elements accurate enough to allow us to extract reliable values of the lepton violating parameters from the data.

As we have mentioned neutrinoless double beta decays (DBD) are concerned with fundamental properties of neutrinos. These properties arise out of interactions involving high energy scales, which are of great interests from view points of particle physics and cosmology. On the other hand, DBD processes are nuclear rare-decays in the low energy scale, which are studied experimentally by low-energy and low-background 
nuclear spectroscopy, as given in review articles [30, 31.

Double beta decays are low-energy second-order weak processes with $Q_{\beta \beta} \approx 2-3$ $\mathrm{MeV}$. Decay rates of $2 \nu \beta \beta$-decay within the SM are of the order of $10^{-20} / \mathrm{y}$, and the rates of $0 \nu \beta \beta$-decay beyond the SM are even many orders of magnitudes smaller than $2 \nu \beta \beta$-decay rates, depending on the $Q_{\beta \beta}$ value and the effective Majorana neutrino mass $\left\langle m_{\nu}\right\rangle$ (for definition see Eq. (33)) in case of the light neutrino mass process. Then the $0 \nu \beta \beta$-decay half lives are of the orders of $T_{1 / 2}^{0 \nu} \approx 10^{27} \mathrm{y}$ and $10^{29} \mathrm{y}$ in cases of the IH (inverted hierarchy) mass of $\left\langle m_{\nu}\right\rangle \approx 30 \mathrm{meV}$ and the $\mathrm{NH}$ (normal hierarchy) mass of $\left\langle m_{\nu}\right\rangle \approx 3 \mathrm{meV}$, respectively.

For experimental studies of such rare decays, large detectors with ton-scale DBD isotopes are needed to get $0 \nu \beta \beta$-decay signals in case of the IH $\nu$ mass. Here the signals are very rare and are as low as $E_{\beta \beta} \approx 2-3 \mathrm{MeV}$. Background (BG) signal rates, however, are huge in the energy region of $E_{B} \leq 3 \mathrm{MeV}$. Thus it is crucial to build ultra low BG detectors to find the rare and small $0 \nu \beta \beta$-decay signals among huge BGs in the low energy region. We are going to review this (see section 8 ) in the case of most of the nuclear targets of experimental interest [30, 31, 132] $\left({ }^{76} \mathrm{Ge},{ }^{82} \mathrm{Se},{ }^{96} \mathrm{Zr},{ }^{100} \mathrm{Mo},{ }^{116} \mathrm{Cd}\right.$, $\left.{ }^{128} \mathrm{Te},{ }^{130} \mathrm{Te},{ }^{136} \mathrm{Xe},{ }^{150} \mathrm{Nd}\right)$.

\section{The neutrino mass matrix in various models}

Within the SM of elementary particles, with the particle content of the gauge bosons $A_{\mu}, Z_{\mu}$ and $W_{\mu}^{ \pm, 0}$, the Higgs scalar isodoublet $\Phi=\left(\phi^{0}, \phi^{-}\right)$(and its conjugate $\Phi^{*}$ ) and the fermion fields arranged in:

- Isodoublets: $\left(u_{\alpha L}, d_{\alpha L}\right)$ and $\left(\nu_{\alpha L}, e_{\alpha L}\right)$ for quarks and leptons respectively and

- Isosinglets: $u_{\alpha R}, d_{\alpha R}$ and $e_{\alpha R}$

where $\alpha$ is a family index taking three values, the neutrinos are massless. They can not obtain mass after the symmetry breaking, like the quarks and the charged leptons, since the right handed neutrino is absent.

\subsection{Neutrino masses at tree level}

The minimal extension of the SM that would yield mass for the neutrino is to introduce an isosinglet right handed neutrino. Then one can have a Dirac mass term arising via coupling of the leptons and Higgs as follows:

$$
\begin{aligned}
& h_{\alpha, \beta}\left(\bar{\nu}_{\alpha_{L}} \bar{e}_{\alpha_{L}}\right)\left(\begin{array}{c}
\phi^{0} \\
\phi^{-}
\end{array}\right) \nu_{\beta R} \rightarrow \\
& h_{\alpha, \beta}\left(\bar{\nu}_{\alpha_{L}} \bar{e}_{\alpha_{L}}\right)\left(\begin{array}{c}
v / \sqrt{2} \\
0
\end{array}\right) \nu_{\beta R} \quad \text { or } \quad m_{\alpha, \beta}^{D}=h_{\alpha, \beta} \frac{v}{\sqrt{2}}
\end{aligned}
$$

Thus one can have:

$$
\mathcal{M}=\left(\bar{\nu}_{L}, \bar{\nu}_{L}^{c}\right)\left(\begin{array}{cc}
0 & m^{D} \\
\left(m^{D}\right)^{T} & 0
\end{array}\right)\left(\begin{array}{l}
\nu_{R} \\
\nu_{R}^{c}
\end{array}\right)
$$


In the above expression, as well as in analogous expressions below, we have explicitly indicated not only the $6 \times 6$ matrix, but the states on which this matrix acts and $m^{D}$ is the above $3 \times 3$ matrix. One then obtains 6 Majorana eigenvectors which pair wise can be associated to opposite eigenvalues. Their sum and their difference may equally well be selected as physical states which correspond to three Dirac neutrinos and their charged conjugate (antineutrinos). This is fine within the above minimal extension. In grand unified theories, however, one is faced with the problem that these neutrinos are going to be very heavy with a mass similar to that of up quarks, which is clearly unacceptable. So such a model is inadequate\&. Besides, such neutrinos viewed as Majorana with opposite $\mathrm{CP}$ eigenvalues or as Dirac particles cannot contribute to $0 \nu \beta \beta$ decay.

The next extension is to introduce a Majorana type mass involving the isosinglet neutrinos and an additional isosinglet Higgs field, which can acquire a large vacuum expectation value, an idea essentially put forward by Weinberg [134] long time ago. Thus the neutrino mass matrix becomes:

$$
\mathcal{M}=\left(\bar{\nu}_{L}, \bar{\nu}_{L}^{c}\right)\left(\begin{array}{cc}
0 & m^{D} \\
\left(m^{D}\right)^{T} & m_{R}
\end{array}\right)\left(\begin{array}{c}
\nu_{R} \\
\nu_{R}^{c}
\end{array}\right)
$$

Thus, provided that the Majorana mass matrix has only very large eigenvalues, one obtains an effective Majorana $3 \times 3$ matrix:

$$
\mathcal{M}_{\nu}=-\bar{\nu}_{L}\left(m^{D}\right)^{T} M_{R}^{-1} m_{D} \nu_{R}^{c}
$$

which can provide small neutrino masses provided that the eigenvalues of the matrix $M_{R}$ are sufficiently large. $M_{R}$ can be arbitrarily large, since the the new scale, associated with the vacuum expectation of the isosinglet, does not affect the low energy scale arising from the vacuum expectation value of the standard Higgs particles. This is the celebrated see-saw mechanism. More precisely the type I see-saw mechanism, since, as we will see below, there exist other see-saw types (for a summary see, e.g., Abada et al[135]).

Thus with the above mechanism the neutrino flavors get admixed, the resulting eigenstates are Majorana particles and lepton number violating interactions, like $0 \nu \beta \beta$ decay, become possible.

Other extensions of the SM are possible, which do not require the addition of the right handed neutrinos but additional exotic scalars or fermions [48, 47], e.g.

- An isotriplet $\Delta$ of Higgs scalars whose charge decomposition is $\delta^{--}, \delta^{-}, \delta^{0}$.

Then this leads to the coupling:

$$
\left(h_{T}\right)_{\alpha, \beta}\left(\bar{\nu}_{\alpha_{L}} \bar{e}_{\alpha_{L}}\right)\left(\begin{array}{cc}
\delta^{-} & -\delta^{0} \\
\delta^{--} & \delta^{-}
\end{array}\right)\left(\begin{array}{c}
e_{\beta R}^{c} \\
-\nu_{\beta R}^{c}
\end{array}\right)
$$

$\S$ There may exist light Dirac neutrinos in theories formulated in extra dimensions, see e.g. the recent review by Smirnov 133. If these neutrinos do not couple to the usual leptons they are of little interest to us. If they do and it so happens that the standard neutrinos are Majorana, they also become Majorana, except in the case of very fine tuning. 


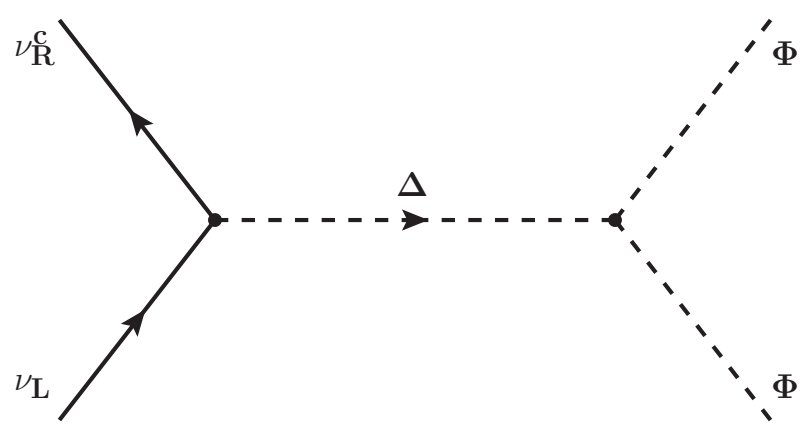

Figure 2: The tree level contribution to the neutrino mass mediated by an isotriplet scalar.

This after the isotriplet acquires a vacuum expectation value becomes

$$
\left(h_{T}\right)_{\alpha, \beta}\left(\bar{\nu}_{\alpha_{L}} \bar{e}_{\alpha_{L}}\right)\left(\begin{array}{rr}
0 & -v_{\Delta} / \sqrt{2} \\
0 & 0
\end{array}\right)\left(\begin{array}{c}
e_{\beta R}^{c} \\
-\nu_{\beta R}^{c}
\end{array}\right)
$$

yielding the neutrino Majorana mass matrix

$$
m_{\alpha, \beta}^{M}=\left(h_{T}\right)_{\alpha, \beta} \frac{v_{\Delta}}{\sqrt{2}} \bar{\nu}_{\alpha_{L}} \nu_{\beta R}^{c}
$$

If, for some reason, the introduction of such an isotriplet, acquiring a small vacuum expectation value, is not preferred, the Majorana mass matrix can be obtained assuming that the isotriplet $\Delta$ possesses a cubic coupling $\mu_{\Delta}$ with two standard Higgs doublets [44, 136, 137, 138] (see Fig. 2). Then one finds an effective Majorana neutrino mass from Eq. (17) via the substitution

$$
\frac{v_{\Delta}}{\sqrt{2}} \rightarrow \frac{v^{2}}{2} \frac{\mu_{\Delta}}{m_{\Delta}^{2}}
$$

where $v / \sqrt{2}$ is the vacuum expectation value of the standard Higgs doublet (see Eq. (13) ), $m_{\Delta}$ is the mass of $\delta^{0}$. This is sometimes refer to as see-saw mechanism II [47, 48].

As we will see later this mechanism may lead to a new contribution to neutrinoless double beta decay via the direct decay of $\delta^{--}$into two electrons.

- An isotriplet of fermions with hypercharge zero $\left(\Sigma^{+}, \Sigma^{0}, \Sigma^{-}\right)$.

In this case the leptons couple to the isotriplet via the higgs doublet( see [139, 140] and references therein):

$$
\begin{gathered}
\left(h_{\Sigma}\right)_{\alpha}\left(\bar{\nu}_{\alpha_{L}} \bar{e}_{\alpha_{L}}\right)\left(\begin{array}{cc}
\Sigma_{R}^{0} / \sqrt{2} & \Sigma_{R}^{+} \\
\Sigma_{R}^{-} & -\Sigma_{R}^{0} / \sqrt{2}
\end{array}\right)\left(\begin{array}{c}
\phi^{0} \\
\phi^{-}
\end{array}\right) \rightarrow \\
\left(h_{\Sigma}\right)_{\alpha}\left(\bar{\nu}_{\alpha_{L}} \bar{e}_{\alpha_{L}}\right)\left(\begin{array}{cc}
\Sigma_{R}^{0} / \sqrt{2} & \Sigma_{R}^{+} \\
\Sigma_{R}^{-} & -\Sigma_{R}^{0} / \sqrt{2}
\end{array}\right)\left(\begin{array}{c}
v / \sqrt{2} \\
0
\end{array}\right)=
\end{gathered}
$$




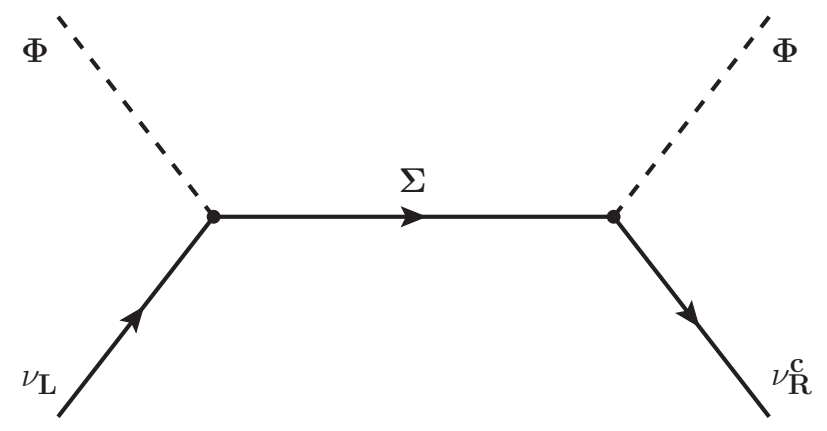

Figure 3: The tree level contribution to the neutrino mass mediated by a fermion isotriplet

$$
\left(h_{\Sigma}\right)_{\alpha} v\left(\frac{1}{2} \bar{\nu}_{\alpha L} \Sigma_{R}^{0}+\frac{1}{\sqrt{2}} \bar{e}_{\alpha L} \Sigma_{R}^{-}\right)
$$

Then a subsequent coupling of the isotriplet to the leptons yields an effective Majorana coupling of the form:

$$
m_{\alpha, \beta}^{M}=-\left(h_{\Sigma}\right)_{\alpha}\left(h_{\Sigma}\right)_{\beta} \frac{v^{2}}{2} \frac{1}{m_{\Sigma}}
$$

where $m_{\Sigma}$ is the mass of the neutral component of the isotriplet (see Fig. 3 and ref. [139]). It is sometimes referred as mechanism see-saw III. This mechanism, however, by itself cannot constitute a viable neutrino mass generator since it leads to two eigenstates with zero mass. This can be circumvented [139, 140], but then the model becomes more complicated.

\subsection{Neutrino masses at the loop level}

There many ways to obtain neutrino masses at the 1-loop level [141], which have nicely been summarized by Smirnov [133]. We will only discuss one such case, which arises in the presence of R-parity violating supersymmetry, which leads to a viable neutrino mass spectrum [142], in the sense that it can yield three massive neutrinos, if one includes not only the tree level contribution arising from the bilinear terms [143], but both type the 1-loop contributions [144, 145] shown in Fig. 4. This is interesting, since, in such models, as we will see below, one can have particles other than neutrinos contributing to $0 \nu \beta \beta$-decay. The above Majorana matrices are symmetric, in general complex, matrices.

\subsection{SUSY, GUT's and Family symmetries}

In many models, like the standard see-saw, the smallness of neutrino mass requires the existence of a heavy mass scale. The coexistence of two mass scales can naturally be accommodated in supersymmetry. In minimal supersymmetric extensions of the SM [146] one construct the see-saw matrix of Eq. (15), see e.g. the review [46]. Furthermore this can be extended to larger symmetries, e.g. two commuting symmetries, a Grand 


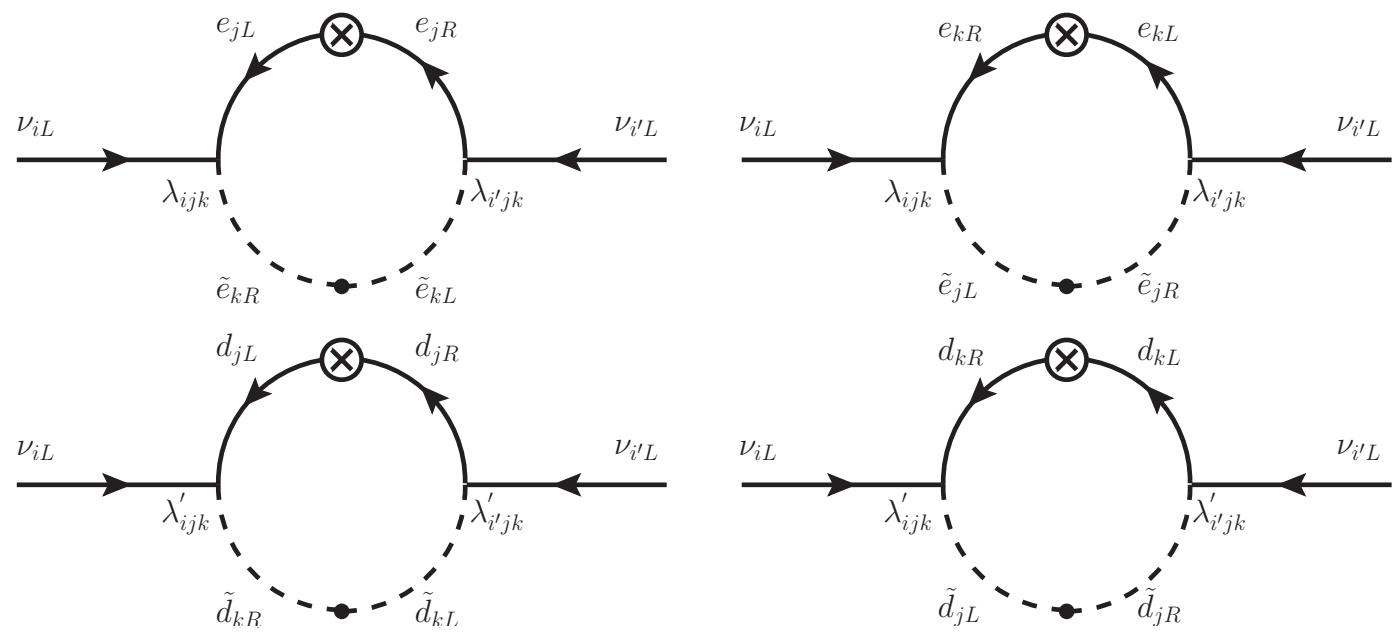

Figure 4: The squark-quark (lower panel) and slepton-lepton (upper panel) 1-loop diagrams contributing to the neutrino mass in the presence of $\mathrm{R}$-parity violating supersymmetric theories.

Unified Symmetry $G_{\mathrm{GUT}}$ and a family symmetry $G_{f}$. The family symmetry could be continuous, like $S U(3), S U(2)$ or $U(1)$ or discreet $Z_{N}, S_{3}$ or $S_{3} \times S_{3}$ etc. We are not going to further elaborate on such situations, which have been summarized in recent reviews [46, 147, 148] to which we direct the interested reader. We should also mention that $U(1)$ flavor symmetries arise naturally in superstring inspired models [149, 150, 151], in particular for the heterotic string and the 4-d fermionic constructions. D-brane models have also paved the way for completely new structures [152, 153, 154, 155] and in particular a very interesting formulation D-brane inspired mass textures [156].

All the models considered in this section lead to a light effective neutrino mass acting jointly or separately. Almost all of them involve parameters, which can be adjusted to fit phenomenology. It is not clear which one, if any, is going to ultimately be the theoretically preferred one.

\subsection{Neutrino mixing}

We have seen above that in general the neutrino mass matrix is a complex symmetric matrix. It can, however, be diagonalized by separate left and write unitary transformations, which can take the form [44]:

$$
\begin{aligned}
& S_{L} \leftrightarrow\left(\nu_{L}^{0}, \nu_{L}^{0 c}\right)=\left(\begin{array}{cc}
S^{(11)} & S^{(12)} \\
S^{(21)} & S^{(11)}
\end{array}\right)\left(\begin{array}{c}
\nu_{L}^{\prime} \\
N_{L}^{\prime}
\end{array}\right), \\
& S_{R} \leftrightarrow\left(\nu_{R}^{0 c}, \nu_{R}^{0}\right)=\left(\begin{array}{ll}
\left(S^{(11)}\right)^{*} & \left(S^{(12)}\right)^{*} \\
\left(S^{(21)}\right)^{*} & \left(S^{(22)}\right)^{*}
\end{array}\right)\left(\begin{array}{c}
\nu_{R}^{\prime} \\
N_{R}^{\prime}
\end{array}\right)
\end{aligned}
$$

where we have added the superscript 0 to stress that they are the states entering the weak interactions. $S^{(i j)}$ are $3 \times 3$ matrices with $S^{(11)}$ and $S^{(22)}$ approximately unitary, 
while $S^{(12)}$ and $S^{(22)}$ are very small. $\left(\nu_{L}^{\prime}, N_{L}^{\prime}\right)$ and $\left(\nu_{R}^{\prime}, N_{R}^{\prime}\right)$ are the eigenvectors from the left and the right respectively. Thus the neutrino mass in the new basis takes the form:

$$
\mathcal{M}_{\nu}=\sum_{j=1}^{3}\left(m_{j} \bar{\nu}_{j L}^{\prime} \nu_{j R}^{\prime}+M_{j} \bar{N}_{j L}^{\prime} N_{j R}^{\prime}\right)+\text { H.C. }
$$

This matrix can be brought into standard form by writing:

$$
m_{j}=\left|m_{i}\right| e^{-i \alpha_{j}}, \quad M_{j}=\left|M_{i}\right| e^{-i \Phi_{j}}
$$

and defining:

$$
\nu_{j}=\nu_{j L}^{\prime}+e^{-i \alpha_{j}} \nu_{j R}^{\prime} \quad N_{j}=\nu_{j L}^{\prime}+e^{-i \Phi_{j}} N_{j R}^{\prime}
$$

Then

$$
\mathcal{M}_{\nu}=\sum_{j=1}^{3}\left(\left|m_{j}\right| \bar{\nu}_{j} \nu_{j}+\left|M_{j}\right| \bar{N}_{j} N_{j}\right)
$$

Note, however, that:

$$
\begin{aligned}
& \nu^{c}=\nu_{j R}^{\prime}+e^{i \alpha_{j}} \nu_{j L}^{\prime}=e^{i \alpha_{j}}\left(\nu_{j L}^{\prime}+e^{-i \alpha_{j}} \nu_{j R}^{\prime}\right)=e^{i \alpha_{j}} \nu_{j} \\
& N^{c}=N_{j R}^{\prime}+e^{i \Phi_{j}} N_{j r}^{\prime}=e^{i \Phi_{j}}\left(\nu_{j R}^{\prime}+e^{-i \Phi_{j}} N_{j R}^{\prime}\right)=e^{i \Phi_{j}} N_{j}
\end{aligned}
$$

i.e. they are Majorana neutrinos with the given Majorana phases. Furthermore

$$
\nu_{i L}=\nu_{i L}^{\prime}, \quad \nu_{i R}=e^{-i \alpha_{j}} \nu_{i R}^{\prime}, \quad N_{i L}=N_{i L}^{\prime}, \quad N_{i R}=e^{-i \Phi_{j}} \nu_{i R}^{\prime}
$$

The second of Eqs. (19) can now be written as

$$
S_{R} \leftrightarrow\left(\nu_{R}^{0 c}, \nu_{R}^{0}\right)=\left(\begin{array}{ll}
\left(S^{(11)}\right)^{*} & \left(S^{(12)}\right)^{*} \\
\left(S^{(21)}\right)^{*} & \left(S^{(22)}\right)^{*}
\end{array}\right)\left(\begin{array}{c}
e^{i \alpha} \nu_{R} \\
e^{i \Phi} N_{R}
\end{array}\right)
$$

where $e^{i \alpha}$ and $e^{i \Phi}$ are diagonal matrices containing the above Majorana phases. The neutrinos interact with the charged leptons via the charged current (see below). So the effective coupling of the neutrinos to the charged leptons involves the mixing of the electrons $S^{e}$. Thus the standard mixing matrix appearing in the absence of right-handed neutrinos is:

$$
U_{P M N S}=U=U^{(11)}=\left(S^{(e)}\right)^{+} S^{(11)}
$$

The other entries are defined analogously:

$$
U^{(i j)}=\left(S^{(e)}\right)^{+} S^{(i j)}, \quad(i j)=(12),(21),(22)
$$

In particular the usual electronic neutrino is written as:

$$
\begin{aligned}
& \nu_{e L}=\sum_{j}\left(U_{e j}^{(11)} \nu_{j}+U_{e j}^{(12)} N_{j}\right) \\
& \nu_{e R}=\sum_{j}\left(U_{e j}^{(21)} \nu_{j}+U_{e j}^{(22)} N_{j}\right)
\end{aligned}
$$


In other words the left handed neutrino may have a small heavy component, while the right handed neutrino may have a small light component. Note also that the neutrinos appearing in weak interactions can be Majorana particles in the special case that all Majorana phases are the same.

The Pontecorvo-Maki-Nakagawa-Sakata neutrino mixing matrix $U_{P M N S}$ is parametrized by

$$
U_{P M N S}=R_{23} \tilde{R}_{13} R_{12}
$$

where the matrices $R_{i j}$ are rotations in $i j$ space, i.e.,

$$
\begin{aligned}
& R_{23}=\left(\begin{array}{lll}
1 & 0 & 0 \\
0 & c_{12} & s_{12} \\
0 & -s_{12} & c_{12}
\end{array}\right), R_{13}=\left(\begin{array}{lll}
c_{13} & 0 & s_{13} e^{-i \delta} \\
0 & 1 & 0 \\
-s_{13} e^{i \delta} & 0 & c_{13}
\end{array}\right) \\
& R_{12}=\left(\begin{array}{lll}
c_{12} & s_{12} & 0 \\
-s_{12} & c_{12} & 0 \\
0 & 0 & 1
\end{array}\right)
\end{aligned}
$$

where

$$
c_{i j} \equiv \cos \left(\theta_{i j}\right), \quad s_{i j} \equiv \sin \left(\theta_{i j}\right) .
$$

$\theta_{12}, \theta_{13}$ and $\theta_{23}$ and three mixing angles and $\delta$ is the CP-violating phase. Then, we get

$$
U_{P M N S}=\left(\begin{array}{lll}
c_{12} c_{13} & c_{13} s_{12} & e^{-i \delta} s_{13} \\
-c_{23} s_{12}-e^{i \delta} c_{12} s_{13} s_{23} & c_{12} c_{23}-e^{i \delta} s_{12} s_{13} s_{23} & c_{13} s_{23} \\
s_{12} s_{23}-e^{i \delta} c_{12} c_{23} s_{13} & -e^{i \delta} c_{23} s_{12} s_{13}-c_{12} s_{23} & c_{13} c_{23}
\end{array}\right),
$$

If neutrinos are Majorana particles $U_{P M N S}$ in Eq. (31) is multiplied by a diagonal phase matrix P, which contains two additional CP-violating Majorana phases $\alpha_{1}$ and $\alpha_{2}$ :

$$
P=\operatorname{diag}\left(e^{i \alpha_{1}}, e^{i \alpha_{2}}, e^{i \delta}\right) .
$$

\section{The elusive absolute scale of the neutrino mass}

With the discovery of neutrino oscillations quite a lot of information regarding the neutrino sector has become available (e.g., for recent reviews see [85, 157]). More specifically we know:

- The mixing angles $\theta_{12}$ and $\theta_{23}$ and we have both a lower and an upper bound on the small angle $\theta_{13}$

- we know the mass squared differences:

$$
\Delta_{\mathrm{SUN}}^{2}=\Delta_{12}^{2}=m_{2}^{2}-m_{1}^{2}, \quad \text { and } \Delta_{\mathrm{ATM}}^{2}=\left|\Delta_{23}^{2}\right|=\left|m_{3}^{2}-m_{2}^{2}\right|
$$

entering the solar and atmospheric neutrino oscillation experiments. Note that we do not know the absolute scale of the neutrino mass and the sign of $\Delta_{23}^{2}$ 
For determination of an absolute scale of the neutrino mass the relevant neutrino oscillation parameters are the MINOS value $\Delta m_{\mathrm{ATM}}^{2}=(2.43 \pm 0.13) \times 10^{-3} \mathrm{eV}^{2}$ [158], the global fit value $\Delta m_{\mathrm{SUN}}^{2}=\left(7.65_{-0.20}^{+0.13}\right) \times 10^{-5} \mathrm{eV}^{2}$ [81], the solar-KamLAND value $\tan ^{2} \theta_{12}=0.452_{-0.033}^{+0.035}$ [159] and the recent Daya Bay observation $\sin ^{2} 2 \theta_{13}=$ $0.092 \pm 0.016$ (stat) \pm 0.005 (syst) with a significance of 5.2 standard deviations [160]. We note that non-zero value of mixing angle $\theta_{13}$ was already observed also by the T2K $\left(0.04<\sin ^{2} 2 \theta_{13}<0.34\right)$ [161], the DOUBLE CHOOZ $\left(\sin ^{2} 2 \theta_{13}=\right.$ $0.085 \pm 0.029$ (stat) \pm 0.042 (syst) $(68 \% \mathrm{CL}))$ [162] and the RENO $\left(\sin ^{2} 2 \theta_{13}=\right.$ $0.103 \pm 0.013$ (stat) \pm 0.011 (syst)) [163] collaborations.

Based on the above we have the following scenarios:

- Normal Spectrum (NS), $m_{1}<m_{2}<m_{3}$ :

$$
\begin{gathered}
\Delta m_{\mathrm{SUN}}^{2}=m_{2}^{2}-m_{1}^{2}, \Delta m_{\mathrm{ATM}}^{2}=m_{3}^{2}-m_{1}^{2} \\
m_{0}=m_{1}, m_{2}=\sqrt{\Delta m_{\mathrm{SUN}}^{2}+m_{0}^{2}}, m_{3}=\sqrt{\Delta m_{\mathrm{ATM}}^{2}+m_{0}^{2}}
\end{gathered}
$$

- Inverted Spectrum (IS), $m_{3}<m_{1}<m_{2}$ :

$$
\begin{gathered}
\Delta m_{\mathrm{SUN}}^{2}=m_{2}^{2}-m_{1}^{2}, \Delta m_{\mathrm{ATM}}^{2}=m_{2}^{2}-m_{3}^{2} \\
m_{0}=m_{3}, m_{2}=\sqrt{\Delta m_{\mathrm{ATM}}^{2}+m_{0}^{2}}, \\
m_{1}=\sqrt{\Delta m_{\mathrm{ATM}}^{2}-\Delta m_{\mathrm{SUN}}^{2}+m_{0}^{2}}
\end{gathered}
$$

The absolute scale $m_{0}$ of neutrino mass can in principle be determined by the following observations:

- Neutrinoless double beta decay.

As we shall see later (section 50) the effective light neutrino mass $\left\langle m_{\nu}\right\rangle$ extracted in such experiments is given as follows [44, 164]:

$$
\begin{aligned}
\left\langle m_{\nu}\right\rangle & =\sum_{k}^{3}\left(U_{e k}^{(11)}\right)^{2} m_{k} \\
& =c_{12}^{2} c_{13}^{2} e^{2 i \alpha_{1}} m_{1}+c_{13}^{2} s_{12}^{2} e^{2 i \alpha_{2}} m_{2}+s_{13}^{2} m_{3} .
\end{aligned}
$$

- The neutrino mass extracted from ordinary beta decay, e.g. from triton decay [165, 166, 167].

$$
\begin{aligned}
\left\langle m_{\nu}\right\rangle_{\text {decay }} & =\sqrt{\sum_{k}^{3}\left|U_{e k}^{(11)}\right|{ }^{2} m_{k}^{2}} \\
& =\sqrt{c_{12}^{2} c_{13}^{2} m_{1}^{2}+c_{13}^{2} s_{12}^{2} m_{2}^{2}+s_{13}^{2} m_{3}^{2}} .
\end{aligned}
$$

assuming, of course, that the three neutrino states cannot be resolved. 
- From astrophysical and cosmological observations (see, e.g., the recent summary[168]).

$$
m_{\nu}=\sum_{k}^{3} m_{k} \leq m_{\text {astro }}
$$

The current limit $m_{\text {astro }}$ depends on the type of observation[168]. Thus CMB primordial gives $1.3 \mathrm{eV}, \mathrm{CMB}+$ distance $0.58 \mathrm{eV}$, galaxy distribution and and lensing of galaxies $0.6 \mathrm{eV}$. On the other hand the largest photometric red shift survey yields $0.28 \mathrm{eV}$ [169]. For purposes of illustration we will take a world average of $m_{\text {astro }}=0.71 \mathrm{eV}$.

The above mass combinations can be written as follows:

(i) Normal Hierarchy (NH), $m_{1} \ll m_{2} \ll m_{3}$.

In this case we have

$$
\Delta m_{\mathrm{SUN}}^{2}=m_{2}^{2}-m_{1}^{2}, \Delta m_{\mathrm{ATM}}^{2}=m_{3}^{2}-m_{1}^{2}
$$

Thus:

- Triton decay.

$$
\begin{aligned}
& \left\langle m_{\nu}\right\rangle_{\text {decay }}= \\
& \sqrt{c_{12}^{2} c_{13}^{2} m_{1}^{2}+s_{12}^{2} c_{13}^{2}\left(\Delta m_{\mathrm{SUN}}^{2}+m_{1}^{2}\right)+s_{13}^{2}\left(\Delta m_{\mathrm{ATM}}^{2}+m_{1}^{2}\right)}
\end{aligned}
$$

- Cosmological bound:

$$
m_{\nu}=m_{1}+\sqrt{\Delta m_{\mathrm{SUN}}^{2}+m_{1}^{2}}+\sqrt{\left(\Delta m_{\mathrm{ATM}}^{2}+m_{1}^{2}\right)}
$$

- $0 \nu \beta \beta$ decay:

$$
\begin{aligned}
\left\langle m_{\nu}\right\rangle=c_{12}^{2} c_{13}^{2} m_{1} e^{2 i \alpha_{1}} & +s_{12}^{2} c_{13}^{2} e^{2 i \alpha_{2}} \sqrt{\Delta m_{\mathrm{SUN}}^{2}+m_{1}^{2}} \\
& +s_{13}^{2} \sqrt{\left(\Delta m_{\mathrm{ATM}}^{2}+m_{1}^{2}\right)}
\end{aligned}
$$

where $\alpha_{1}$ and $\alpha_{2}\left(\alpha_{3}=0\right)$ are Majorana $\mathrm{CP}$ violating phases of the elements $U_{e 1}$ and $U_{e 2}$ with $U_{e j}=\left|U_{e j}\right| e^{i \alpha_{j}}(i=1,2)$.

By assuming $\mathrm{NH}$, i.e., that $m_{1}$ is negligibly small $\left(m_{1} \ll \sqrt{\Delta m_{\text {SUN }}^{2}}, m_{2} \simeq\right.$ $\sqrt{\Delta m_{\mathrm{SUN}}^{2}}$, and $\left.m_{3} \simeq \sqrt{\Delta m_{\mathrm{ATM}}^{2}}\right)$, we obtain

$$
\begin{aligned}
\left|\left\langle m_{\nu}\right\rangle\right| & \simeq\left|c_{13}^{2} s_{12}^{2} \sqrt{\Delta m_{\mathrm{SUN}}^{2}}+s_{13}^{2} \sqrt{\Delta m_{\mathrm{ATM}}^{2}} e^{-2 i \alpha_{2}}\right| \\
& \leq 4 \cdot 10^{-3} \mathrm{eV} .
\end{aligned}
$$

(ii) Inverted Hierarchy (IH), $m_{3} \ll m_{1}<m_{2}$ : In this case we have

$$
\Delta m_{\mathrm{SUN}}^{2}=m_{2}^{2}-m_{1}^{2}, \Delta m_{\mathrm{ATM}}^{2}=m_{2}^{2}-m_{3}^{2}
$$

- Triton decay.

$$
\begin{aligned}
\left\langle m_{\nu}\right\rangle_{\text {decay }}= & \left(s_{13}^{2} m_{3}^{2}+s_{12}^{2} c_{13}^{2}\left(\Delta m_{\mathrm{ATM}}^{2}+m_{3}^{2}\right)\right. \\
& \left.+c_{12}^{2} c_{13}^{2}\left(\Delta m_{\mathrm{ATM}}^{2}-\Delta m_{\mathrm{SUN}}^{2}+m_{3}^{2}\right)\right)^{1 / 2}
\end{aligned}
$$



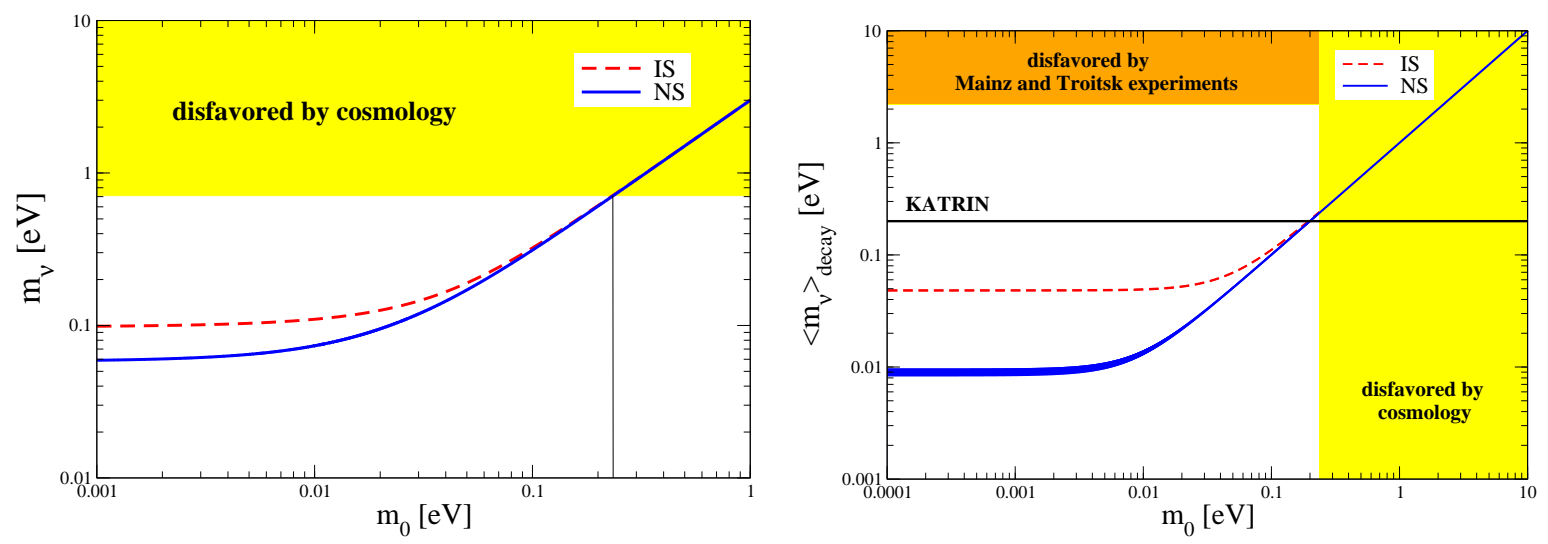

Figure 5: (Color online) The neutrino mass limits in $\mathrm{eV}$ as a function of mass of the lowest eigenstate $m_{0}$ also in $\mathrm{eV}$, extracted from cosmology (left panel) triton decay (right panel). From the current upper limit of $2.2 \mathrm{eV}$ of the Mainz and Troitsk experiments we deduce a lowest neutrino mass of $2.2 \mathrm{eV}$ both for the NS and IS. From the astrophysical limit value of $0.71 \mathrm{eV}$ the corresponding neutrino mass extracted is about $0.23 \mathrm{eV}$ for the NS and IS. It is assumed: $\Delta m_{\mathrm{ATM}}^{2}=(2.43 \pm 0.13) \times 10^{-3} \mathrm{eV}^{2}$ [158], $\Delta m_{\mathrm{SUN}}^{2}=$ $\left(7.65_{-0.20}^{+0.13}\right) \times 10^{-5} \mathrm{eV}^{2}$ [81], $\tan ^{2} \theta_{12}=0.452_{-0.033}^{+0.035}$ [159] and $\sin ^{2} 2 \theta_{13}=0.092 \pm 0.016$ [160].

- Cosmological bound:

$$
m_{\nu}=m_{3}+\sqrt{\left(\Delta m_{\mathrm{ATM}}^{2}+m_{3}^{2}\right)}+\sqrt{\left(\Delta m_{\mathrm{ATM}}^{2}-\Delta m_{\mathrm{SUN}}^{2}+m_{3}^{2}\right)}
$$

- $0 \nu \beta \beta$ decay:

$$
\begin{aligned}
\left\langle m_{\nu}\right\rangle=s_{13}^{2} m_{3} & +s_{12}^{2} c_{13}^{2} e^{2 i \alpha_{2}} \sqrt{\left(\Delta m_{\mathrm{ATM}}^{2}+m_{3}^{2}\right)} \\
& +c_{12}^{2} c_{13}^{2} e^{2 i \alpha_{1}} \sqrt{\left(\Delta m_{\mathrm{ATM}}^{2}-\Delta m_{\mathrm{SUN}}^{2}+m_{3}^{2}\right)}
\end{aligned}
$$

Since in IH scenario $m_{3}$ is negligibly small $\left(m_{1} \simeq m_{2} \simeq \sqrt{\Delta m_{\mathrm{Atm}}^{2}}\right.$ and $\left.m_{3} \ll \sqrt{\Delta m_{\text {ATM }}^{2}}\right)$, we find

$$
\left|\left\langle m_{\nu}\right\rangle\right| \simeq \sqrt{\Delta m_{\mathrm{Atm}}^{2}} c_{13}^{2}\left(1-\sin ^{2} 2 \theta_{12} \sin ^{2} \alpha_{12}\right)^{\frac{1}{2}}
$$

where $\alpha_{12}=\alpha_{2}-\alpha_{1}$. The phase difference $\alpha_{12}$ is the only unknown parameter in the expression for $\left|\left\langle m_{\nu}\right\rangle\right|$. From (43) we obtain the following inequality [170]

$$
1.5 \cdot 10^{-2} \mathrm{eV} \leq\left|\left\langle m_{\nu}\right\rangle\right| \leq 5.0 \cdot 10^{-2} \mathrm{eV} .
$$

(iii) Quasi-degenerate (QD) spectrum, $m_{0}=m_{1} \simeq m_{2} \simeq m_{3}$. Then

- Triton decay and Cosmology:

$$
\left\langle m_{\nu}\right\rangle_{\text {decay }}=m_{0}, \quad m_{\nu}=3 m_{0}
$$

- $0 \nu \beta \beta$ decay:

The effective Majorana mass is relatively large in this case and for both types of the neutrino mass spectrum is given by the expression

$$
m_{0}\left|1-2 c_{13}^{2} c_{12}^{2}\right| \leq\left|\left\langle m_{\nu}\right\rangle\right| \leq m_{0} .
$$


The above results are exhibited in Fig. 5 for the tritium $\beta$-decay and cosmological limits as a function of the lowest neutrino mass and in Fig. 6 for the case of the $0 \nu \beta \beta$ decay both for the NS and the IS scenarios. The allowed range values of $\left|\left\langle m_{\nu}\right\rangle\right|$ as a function of the lowest mass eigenstate $m_{0}$ is exhibited. For the values of the parameter $\sin ^{2} 2 \theta_{13}$ new Double Chooz data are used [162]. The IH allowed region for $\left|\left\langle m_{\nu}\right\rangle\right|$ is presented by the region between two parallel lines in the upper part of Fig. 6. The $\mathrm{NH}$ allowed region for $\left|\left\langle m_{\nu}\right\rangle\right| \approx$ few meV is compatible with $m_{0}$ smaller than $10 \mathrm{meV}$. The quasi-degenerate spectrum can be determined, if $m_{0}$ is known from future $\beta$-decay experiments KATRIN [165, 166] and MARE [167] or from cosmological observations. The lowest value for the sum of the neutrino masses, which can be reached in future cosmological measurements [171, 172, 173], is about (0.05-0.1) eV. The corresponding values of $m_{0}$ are in the region, where the IS and the NS predictions for $\left|\left\langle m_{\nu}\right\rangle\right|$ differ significantly from each other.

From the most precise experiments on the search for $0 \nu \beta \beta$-decay [36, 39, 40] by using of nuclear matrix elements of Ref. [174] the following stringent bounds were inferred (see Table 1)

$$
\begin{aligned}
\left|\left\langle m_{\nu}\right\rangle\right| & <(0.20-0.32) e V \quad\left({ }^{76} \mathrm{Ge}\right) \\
& <(0.33-0.46) e V \quad\left({ }^{130} \mathrm{Te}\right) \\
& <(0.17-0.30) e V \quad\left({ }^{136} \mathrm{Xe}\right)
\end{aligned}
$$

These bounds we obtained using the $0 \nu \beta \beta$-decay NMEs of [174] calculated with Brueckner two-nucleon short-range correlations. There exist, however, a claim of the observation of the $0 \nu \beta \beta$-decay of ${ }^{76} \mathrm{Ge}$ made by some participants of the HeidelbergMoscow collaboration [42]. Their estimated value of the effective Majorana mass (assuming a specific value for the NME) is $\left|\left\langle m_{\nu}\right\rangle\right| \simeq 0.4 \mathrm{eV}$. This result will be checked by an independent experiment relatively soon. In the new germanium experiment GERDA [43, 175], the Heidelberg-Moscow sensitivity will be reached in about one year of measuring time.

In future experiments, CUORE[176], EXO[177, 178], MAJORANA[179], SuperNEMO [180], SNO+ [181], KamLAND-Zen and others [6, 31, 182], a sensitivity

$$
\left|\left\langle m_{\nu}\right\rangle\right| \simeq \text { a few } 10^{-2} \mathrm{eV}
$$

is planned to be reached, what is the region of the IH of neutrino masses. In the case of the normal mass hierarchy $\left|\left\langle m_{\nu}\right\rangle\right|$ is too small in order to be probed in the $0 \nu \beta \beta$-decay experiments of the next generation.

Recently, however, for the explanation of the Reactor Antneutrino Anomaly [185], a light sterile neutrino has been introduced with mass squared difference:

$$
\Delta m_{24}^{2}=\left|m_{2}^{2}-m_{4}^{2}\right| \approx \Delta m_{14}^{2}=\left|m_{1}^{2}-m_{4}^{2}\right| \geq 1.5(\mathrm{eV})^{2}
$$

which couples to the electron neutrino with a mixing angle:

$$
\sin ^{2} 2 \theta_{14}=0.14 \pm 0.08 \text { (95\% C.L.). }
$$




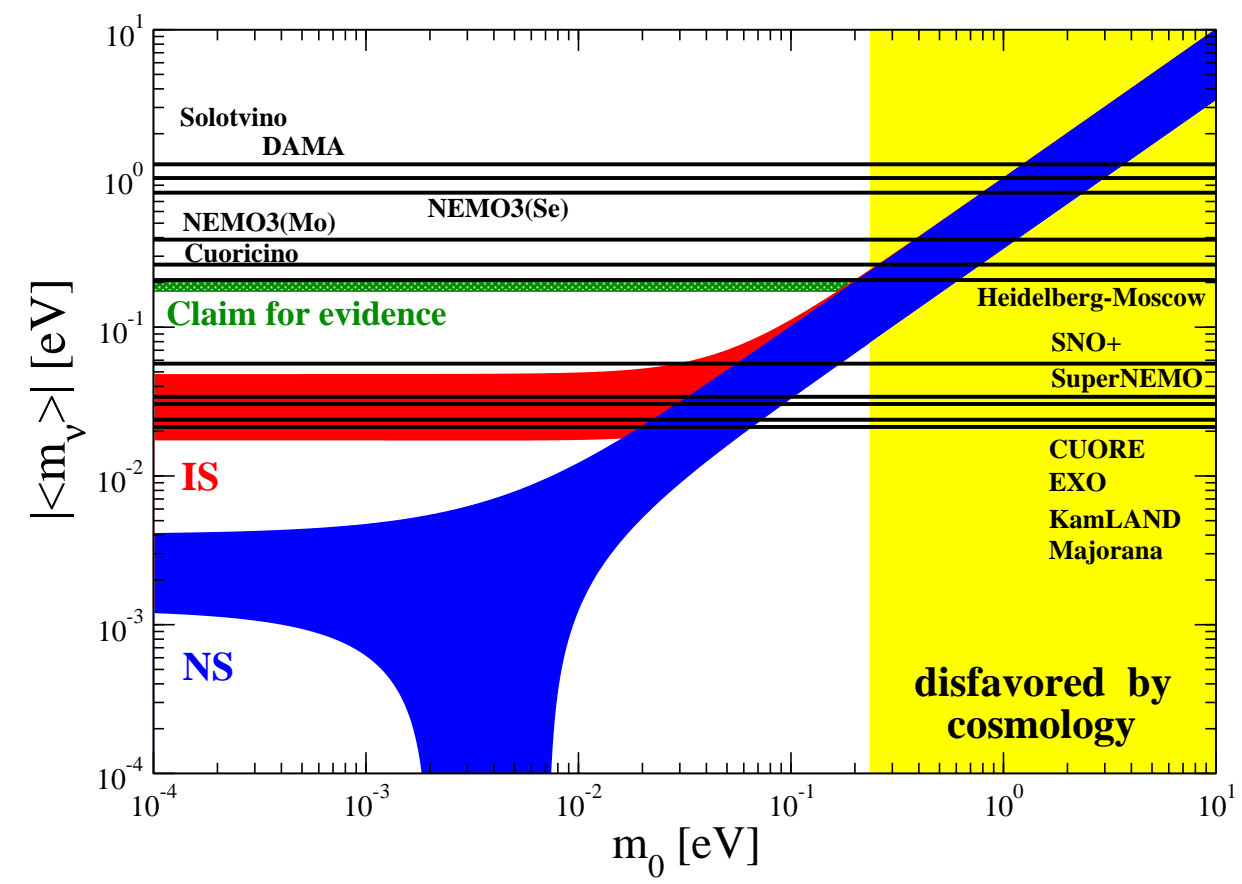

Figure 6: (Color online) We show the allowed range of values for $\left|\left\langle m_{\nu}\right\rangle\right|$ as a function of the lowest mass eigenstate $m_{0}$ using the three standard neutrinos for the cases of normal (NS, $m_{0}=m_{1}$ ) and inverted (IS, $m_{0}=m_{3}$ ) spectrum of neutrino masses. Also shown are the current experimental limits and the expected future results [183] (QRPA NMEs with CD-Bonn short-range correlations and $g_{A}^{e f f}=1.25$ are assumed [174, 184]). Note that in the inverted hierarchy there is a lower bound, which means that in such a scenario the $0 \nu \beta \beta$-decay should definitely be observed, if the experiments reach the required level. The same set of neutrino oscillation parameters as in Fig. 5 is considered.

On the other hand in a recent global analysis more than one sterile neutrino are needed [186], with somewhat smaller mass squared differences, but similar couplings. Due to such a mixing, even if their couplings are of the usual Dirac type, the resulting mass eigenstates are of the Majorana type due to their coupling to the usual neutrino.

The $U(4 \times 4)$ neutrino mixing matrix in the presence of one sterile neutrino with a small mixing becomes [187]:

$$
U=R_{34} \tilde{R}_{24} \tilde{R}_{14} R_{23} \tilde{R}_{13} R_{12} P .
$$

It depends on 6 mixing angles $\left(\theta_{14}, \theta_{24}, \theta_{34}, \theta_{12}, \theta_{13}, \theta_{23}, 3\right.$ Dirac $\left(\delta_{24}, \delta_{14}, \delta_{13}\right)$ and 3 Majorana $\left(\alpha_{1}, \alpha_{2}, \alpha_{3}\right)$ CP-violating phases entering the diagonal P matrix :

$$
P=\operatorname{diag}\left(e^{i \alpha_{1}}, e^{i \alpha_{2}}, e^{i\left(\alpha_{3}+\delta_{13}\right)}, e^{i \delta_{14}}\right) .
$$

Similarly, one can parametrized the $U(5 \times 5)$ mixing matrix for two sterile neutrinos as (10 mixing angles and $5+4 \mathrm{CP}$ violating phases)

$$
U=R_{45} \tilde{R}_{35} R_{34} \tilde{R}_{25} \tilde{R}_{24} R_{23} \tilde{R}_{15} \tilde{R}_{14} \tilde{R}_{13} R_{12} P
$$

where $P=\operatorname{diag}\left(e^{i \alpha_{1}}, e^{i \alpha_{2}}, e^{i\left(\alpha_{3}+\delta_{13}\right)}, e^{i\left(\alpha_{4}+\delta_{14}\right)}, e^{i \delta_{15}}\right)$. 


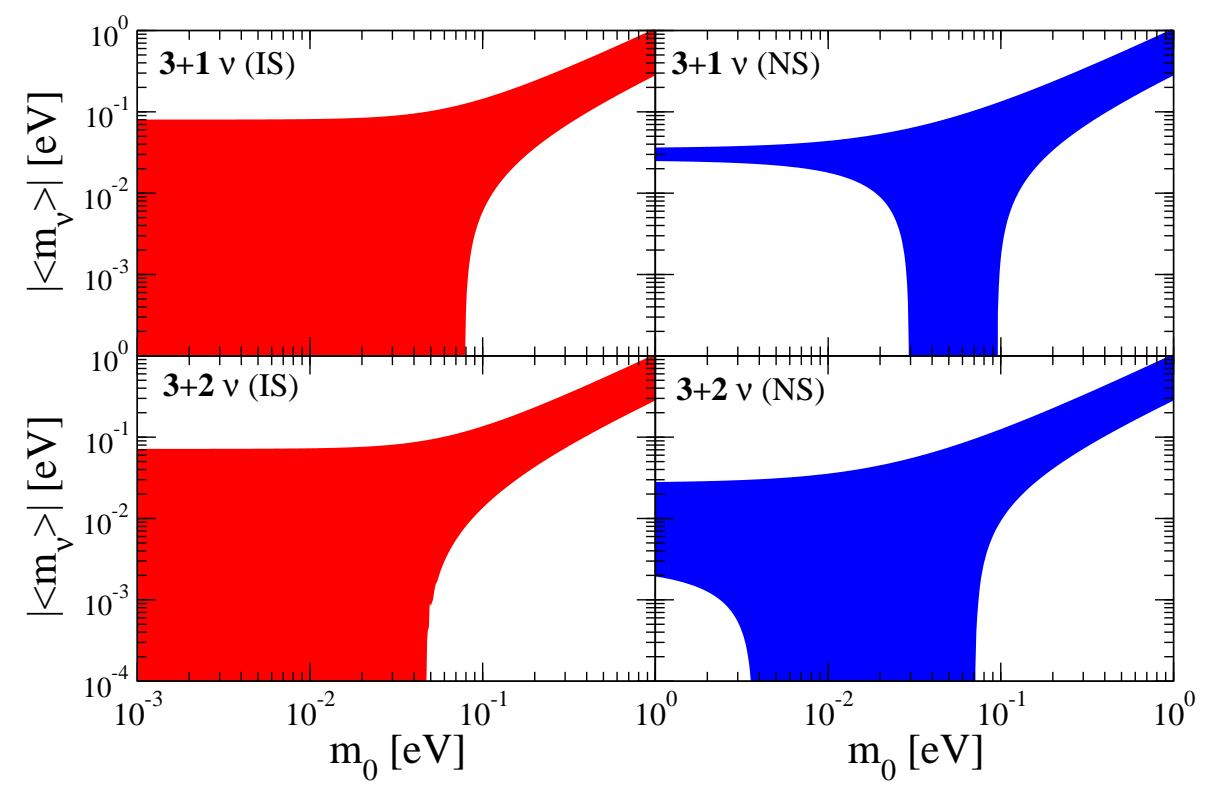

Figure 7: (Color online) The same as in Fig. 6, if one considers one (version 3+1) or two (version 3+2) sterile neutrinos, which are heavier than the standard neutrinos. Best fit points for the $3+1\left(\Delta m_{41}^{2}=1.78 \mathrm{eV}^{2}, U_{e 4}=0.151\right)$ and $3+2\left(\Delta m_{41}^{2}=0.46 \mathrm{eV}^{2}\right.$, $U_{e 4}=0.108$ and $\left.\Delta m_{51}^{2}=0.89 \mathrm{eV}^{2}, U_{e 5}=0.124\right)$ scenarios from reactor antineutrino data are taken into account [186]. In addition, best fit values $\Delta m_{\mathrm{ATM}}^{2}=2.43 \times 10^{-3} \mathrm{eV}^{2}$ [158], $\Delta m_{\mathrm{SUN}}^{2}=7.65 \times 10^{-5} \mathrm{eV}^{2}$ [81], $\tan ^{2} \theta_{12}=0.452$ [159] and $\sin ^{2} 2 \theta_{13}=0.092 \pm 0.016$ [160] are assumed.

If the presence of one sterile neutrino, the effective neutrino mass in the $0 \nu \beta \beta$-decay is given by [187]

$$
\begin{aligned}
\left|\left\langle m_{\nu}\right\rangle_{3+1}\right|= & \mid c_{12}^{2} c_{13}^{2} c_{14}^{2} e^{2 i \alpha_{1}} m_{1}+c_{13}^{2} c_{14}^{2} s_{12}^{2} e^{2 i \alpha_{2}} m_{2} \\
& +c_{14}^{2} s_{13}^{2} e^{2 i \alpha_{3}} m_{3}+s_{14}^{2} m_{4} \mid .
\end{aligned}
$$

We note that from three additional angles $\left\langle m_{\nu}\right\rangle \mid$ depends only one of them, namely $\theta_{14}$. If there are two sterile neutrinos we end up with

$$
\begin{aligned}
\left|\left\langle m_{\nu}\right\rangle_{3+2}\right|=\mid & \mid c_{12}^{2} c_{13}^{2} c_{14}^{2} c_{15}^{2} e^{2 i \alpha_{1}} m_{1}+c_{13}^{2} c_{14}^{2} c_{15}^{2} s_{12}^{2} e^{2 i \alpha_{2}} m_{2} \\
& +c_{14}^{2} c_{15}^{2} s_{13}^{2} e^{2 i \alpha_{3}} m_{3}+c_{15}^{2} s_{14}^{2} e^{2 i \alpha_{4}} m_{4}+s_{15}^{2} m_{5} \mid .
\end{aligned}
$$

Due to the extra terms in Eqs. (54) and (55) and their couplings, depending on the extra majorana phases, the sterile could dominate, increase or deplete $\left\langle m_{\nu}\right\rangle$. By assuming best fit values for $\left|U_{e 4}\right|, m_{41}^{2}$ and $\left|U_{e 5}\right|, m_{51}^{2}$ from reactor antineutrino data [186] the allowed range values of $\left\langle m_{\nu}\right\rangle$ as a function as a function of the lowest mass eigenstate $m_{0}$ in the presence of one and two sterile neutrinos is shown in Fig. 7. 


\section{The intermediate Majorana neutrino mechanism in $0 \nu \beta \beta$ decay}

To proceed further in our study of the neutrino mediated $0 \nu \beta \beta$-decay process it is necessary to study the structure the effective weak beta decay Hamiltonian. In general it has both left handed and right handed components. Within the $S U(2)_{L} \times S U(2)_{R} \times U(1)$ at low energies it takes the current-current form:

$$
\begin{aligned}
\mathcal{H}^{\beta}=\frac{G_{F}}{\sqrt{2}} 2 & {\left[\left(\bar{e}_{L} \gamma_{\mu} \nu_{e L}^{0}\right)\left(J_{L}^{\mu \dagger}+\epsilon J_{R}^{\mu \dagger}\right)\right.} \\
+ & \left.\left(\bar{e}_{R} \gamma_{\mu} \nu_{e R}^{0}\right)\left(\epsilon J_{L}^{\mu \dagger}+\kappa J_{R}^{\mu \dagger}\right)+\text { h.c. }\right],
\end{aligned}
$$

Here, $\epsilon$ is mixing of $W_{L}$ and $W_{R}$ gauge bosons

$$
W_{L}=\cos \epsilon W_{1}-\sin \epsilon W_{2}, \quad W_{R}=\sin \epsilon W_{1}+\cos \epsilon W_{2}
$$

where $W_{1}$ and $W_{2}$ are the mass eigenstates of the gauge bosons with masses $M_{W_{1}}$ and $M_{W_{2}}$, respectively. The mixing is assumed to be small, $\sin \epsilon \approx \epsilon, \cos \epsilon \approx 1$, and $m_{W_{1}} \approx m_{W_{L}}, m_{W_{2}} \approx m_{W_{R}} . \kappa$ is the mass squared ratio

$$
\kappa=\frac{m_{W_{1}}^{2}}{m_{W_{2}}^{2}} .
$$

$J_{L}^{\mu}$ is the standard hadronic current in V-A theory:

$$
J_{L}^{\mu \dagger}=\sum_{i} \bar{u}_{p}(i)\left[g_{V} \gamma^{\mu}+i g_{M} \frac{\sigma^{\mu \nu}}{2 m_{p}} q_{\nu}-g_{A} \gamma^{\mu} \gamma_{5}-g_{P} q^{\mu} \gamma_{5}\right] u_{n}(i),
$$

where $u_{p}(i)$ and $u_{n}(i)$ are the spinors describing the proton and neutron $i . m_{p}$ is the nucleon mass and $q^{\mu}$ is the momentum transfer. $g_{V} \equiv g_{V}\left(q^{2}\right), g_{M} \equiv g_{M}\left(q^{2}\right), g_{A} \equiv g_{A}\left(q^{2}\right)$ and $g_{P} \equiv g_{P}\left(q^{2}\right)$ are respectively the vector, weak-magnetism, axial-vector and induced pseudoscalar form-factors.

We will see below that it is necessary to also consider the right handed current $J_{R}^{\mu \dagger}$ of the form $\mathrm{V}+\mathrm{A}$,

$$
J_{R}^{\mu \dagger}=\sum_{i} \bar{u}_{p}(i)\left[g_{V} \gamma^{\mu}+i g_{M} \frac{\sigma^{\mu \nu}}{2 m_{p}} q_{\nu}+g_{A} \gamma^{\mu} \gamma_{5}+g_{P} q^{\mu} \gamma_{5}\right] u_{n}(i),
$$

even though normally one expects its contribution to ordinary beta decay to be suppressed by a factor $\kappa$ or $\epsilon$. Some relations among form factors in $J_{L}^{\mu \dagger}$ and $J_{R}^{\mu \dagger}$ are considered because the strong and electromagnetic interactions conserve parity.

$e_{L}\left(e_{R}\right)$ and $\nu_{e L}^{0}\left(\nu_{e R}^{0}\right)$ are field operators representing the left (right) handed electrons and electron neutrinos in a weak interaction basis in which the charged leptons are diagonal. We have seen above the the weak neutrino eigenstates can be expressed in terms of the propagating mass eigenstates [44] (see Eqs. (26) and (27)). The mass eigenstates $\nu_{k}, N_{k}$ satisfy the Majorana condition: $\nu_{k} \xi_{k}=C \bar{\nu}_{k}^{T}, N_{k} \Xi_{k}=C \bar{N}_{k}^{T}$, where $\mathrm{C}$ denotes the charge conjugation and $\xi, \Xi$ are phase factors, which guarantee that the eigenmasses are positive $\left(\xi_{k}=e^{i \alpha_{k}}, \Xi_{k}=e^{i \Phi_{k}}\right.$, see Eq. (221) $)$. 


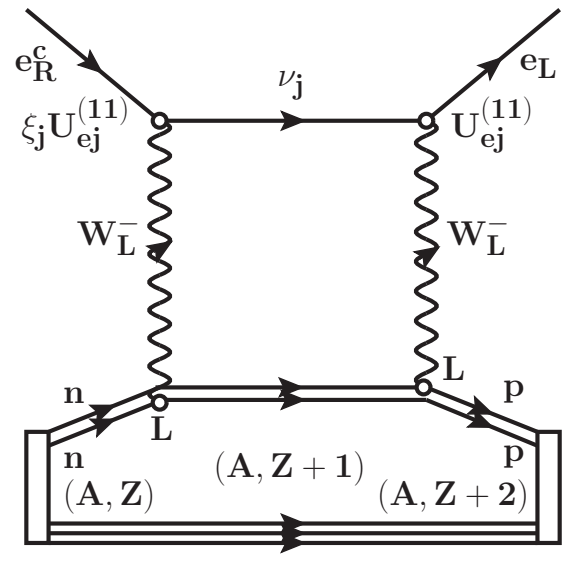

(a)

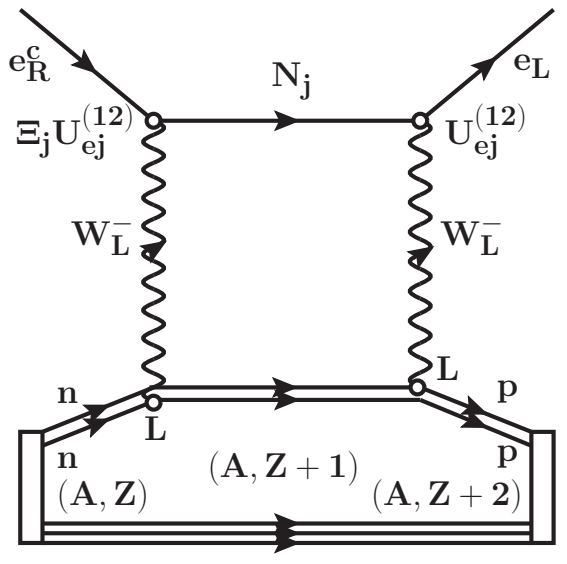

(b)

Figure 8: The neutrino mass contribution at the nuclear level in the presence of left handed currents for light intermediate neutrino (a) and heavy neutrino (b).

Before proceeding further we should mention that in the context of the above interaction neutrinoless double beta decay is a two step process. The neutrino is produced via the lepton current in one vertex and propagates in the other vertex. If the two current helicities are the same one picks out of the neutrino propagator the term:

$$
\frac{m_{j}}{q^{2}-m_{j}^{2}} \rightarrow\left\{\begin{array}{cc}
m_{j} / q^{2}, & m_{j}^{2} \ll q^{2} \\
-1 / m_{j}, & m_{j}^{2} \gg q^{2}
\end{array}\right.
$$

where $q$ is the momentum transferred by the neutrino. In other words the amplitude for light neutrino becomes proportional to its mass, but for a heavy neutrino inversely proportional to its mass.

If the leptonic currents have opposite chirality the one picks out of the neutrino propagator the term:

$$
\frac{q}{q^{2}-m_{j}^{2}} \rightarrow \frac{q}{q^{2}}, \quad m_{j}^{2} \ll q^{2}
$$

i.e. in the interesting case of light neutrino the amplitude does not explicitly depend on the neutrino mass. The kinematics becomes different than that for the mass term.

\subsection{The Majorana neutrino mass mechanism}

This mechanism is the most popular and most commonly discussed in the literature (see Fig. 8). From this figure we can see read off the couplings and the phases. We have also seen that for currents of the same chirality one picks out the mass of the propagating neutrino. Thus the lepton violating parameter is defined as $\left\langle m_{\nu}\right\rangle / m_{e}$ with $\left\langle m_{\nu}\right\rangle$ given by Eq. (33).

We will consider only $0_{i}^{+} \rightarrow 0_{f}^{+}$transitions. Then both outgoing electrons are in the $s_{1 / 2}$ state. Thus for the ground state transition, restricting ourselves to the usual 


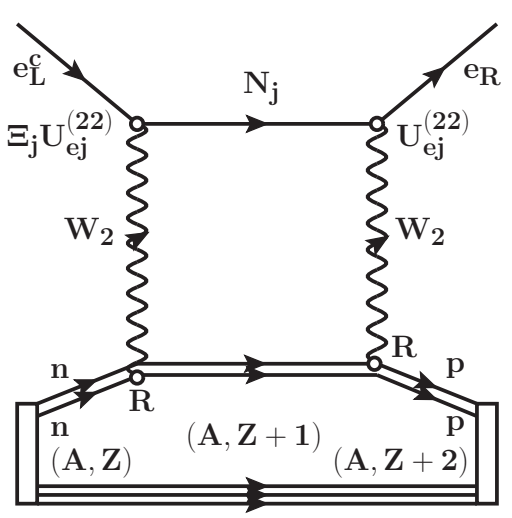

(a)

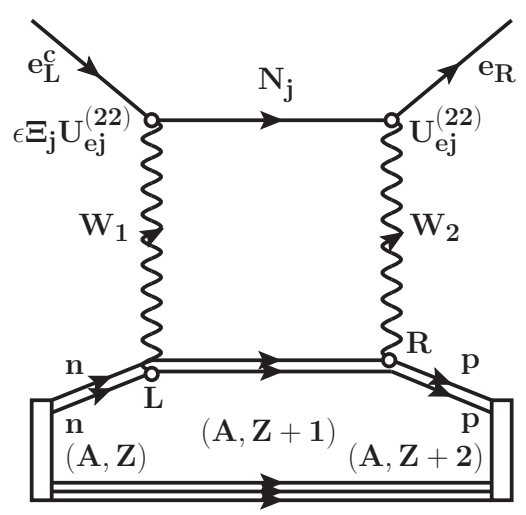

(c)

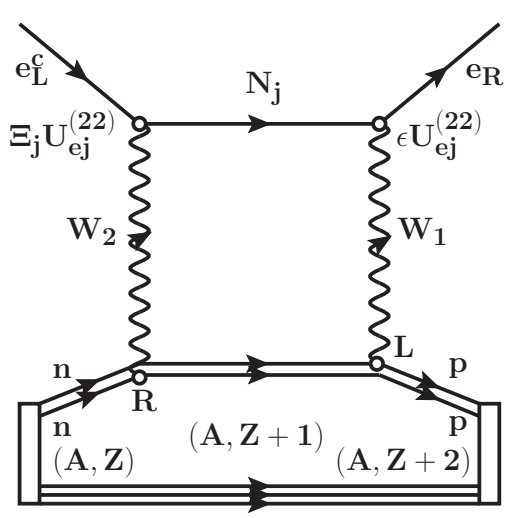

(b)

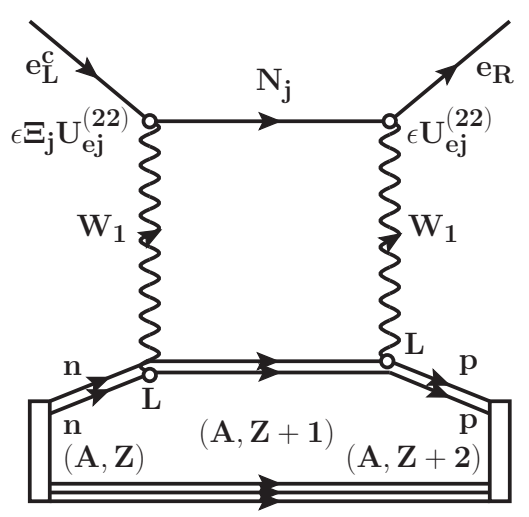

(d)

Figure 9: The neutrino mass contribution at the nuclear level in the presence of right handed currents proceeding via the right handed boson (a) ( $\kappa^{2}$ suppression factor), the combination of the right handed boson and the mixing between the two bosons (b) and (c) $(\kappa \times \epsilon$ suppression factor) and via the light gauge boson via its mixing with its heavy partner $(d)$ ( $\epsilon^{2}$ suppression factor).

light left handed neutrino mass mechanism, we obtain the following expression for the $0 \nu \beta \beta$-decay inverse half-life:

$$
\left[T_{1 / 2}^{0 \nu}\right]^{-1}=G_{01}\left|\frac{\left\langle m_{\nu}\right\rangle}{m_{e}}\right|^{2}\left|M_{\nu}^{0 \nu}\right|^{2}
$$

Extraction of $\left\langle m_{\nu}\right\rangle$ from the above life time will have a wide range of implications in physics, it can, e.g., constrain the baryon asymmetry in the universe [188] $\left|Y_{B}\right|$ etc.

Another less popular possibility is the mass contribution arising from DBD in the presence of right handed currents (see Fig. 9) or heavy neutrinos in general[189]. The above expression can be generalized to include many mass mechanisms [44, 62, 63, 64, 65, 66, 87] as follows:

$$
\left[T_{1 / 2}^{0 \nu}\right]^{-1}=G_{01}\left[\left|X_{L}\right|^{2}+\left|X_{R}\right|^{2}-\tilde{C}_{1}^{\prime} X_{L} X_{R}+\ldots\right] .
$$


The coefficient $\tilde{C}_{1}^{\prime}$ is negligible, because these terms do not interfere to leading order due to the different helicity of the 2 electrons. Here ... indicate other non traditional modes (SUSY etc.). The nuclear matrix elements entering the above expression are given in units of $M_{G T}$ and are denoted [63] by $\chi$.

$$
X_{L}=\frac{\left\langle m_{\nu}\right\rangle}{m_{e}} M_{\nu}^{0 \nu}+\eta_{N}^{L} M_{N}^{0 \nu}, \quad X_{R}=\eta_{N}^{R} M_{N}^{0 \nu},
$$

where the nuclear matrix elements $M_{\nu}^{0 \nu}$ and $M_{N}^{0 \nu}$ will be discussed later (see section 9). The subscripts $L(R)$ indicate left (right) handed currents respectively. The leptonnumber non-conserving parameters, i.e. the effective neutrino mass $\left\langle m_{\nu}\right\rangle$ given by Eq. (33) and $\eta_{N}^{L}, \eta_{N}^{R}$ are given as follows [44]:

$$
\begin{aligned}
\eta_{N}^{L} & =\sum_{k}^{3}\left(U_{e k}^{(12)}\right)^{2} \Xi_{k} \frac{m_{p}}{M_{k}}, \\
\eta_{N}^{R} & =\left(\kappa^{2}+\epsilon^{2}+2 \epsilon \kappa\right) \sum_{k}^{3}\left(U_{e k}^{22}\right)^{2} \quad \Xi_{k} \frac{m_{p}}{M_{k}},
\end{aligned}
$$

with $m_{p}\left(m_{e}\right)$ being the proton (electron) mass. $G_{01}$ is the integrated kinematical factor [53, 51, 63, 87]. The nuclear matrix elements associated with the exchange of light and heavy neutrino must be computed in a suitable nuclear model. The ellipses ... mean that Eq. (64) can be generalized to the mass term resulting from any other intermediate fermion.

At this point we should stress that the main suppression in the case of light neutrinos comes from the smallness of neutrino masses. In the case of heavy neutrino not only from the large values of neutrino masses but the small couplings, $U^{(12)}$ for the left handed neutrinos and $\kappa$ and $\epsilon$ for the right-handed ones.

\subsection{The neutrino mass independent mechanism (leptonic left-right interference, $\lambda$ and} $\eta$ terms).

As we have already mentioned in the presence of right handed currents one can have interference between the leptonic currents of opposite chirality (see Fig. 10). The elementary amplitude is now proportional to the 4-momentum transfer. We thus have a space component and a time component in the relevant amplitude. This leads to different kinematical functions and to two new lepton number violating parameters [44] $\langle\lambda\rangle$ and $\langle\eta\rangle$ defined by

$$
\langle\eta\rangle=\epsilon \eta_{R L}, \quad\langle\lambda\rangle=\kappa \eta_{R L}, \quad \eta_{R L}=\sum_{1}^{3}\left(U_{e k}^{(21)} U_{e k}^{(11)}\right) \xi_{k} .
$$

The parameters $\langle\lambda\rangle$ and $\langle\eta\rangle$ are small not only due to the smallness of the parameters $\kappa$ and $\epsilon$ but, in addition, due to the smallness of $U^{(21)}$. As we have already mentioned the $\langle\lambda\rangle$ can also have a different origin (see section 6.2.4).

All the above contributions, even though the relevant amplitudes are not explicitly dependent on the neutrino mass, vanish in the limit in which the neutrino is a Dirac particle. 


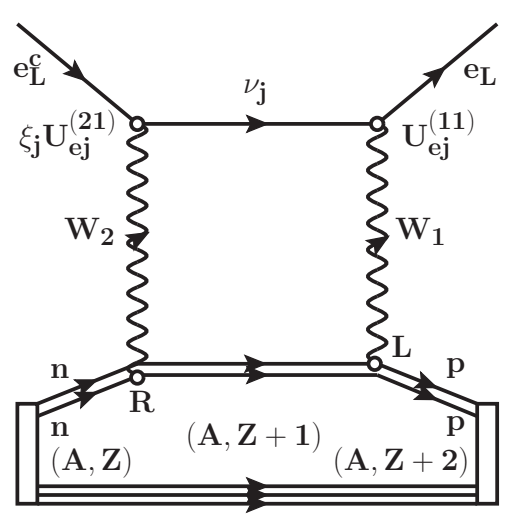

(a)

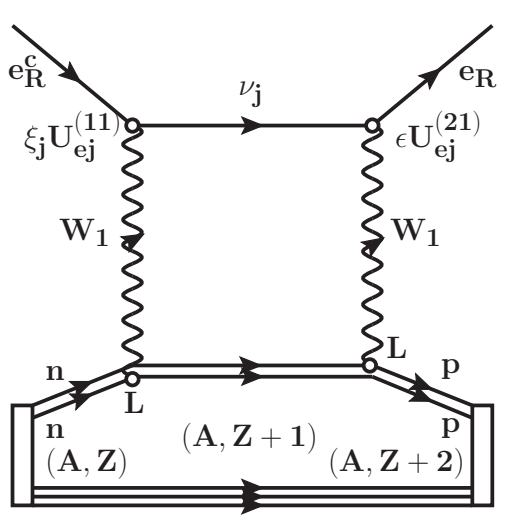

(c)

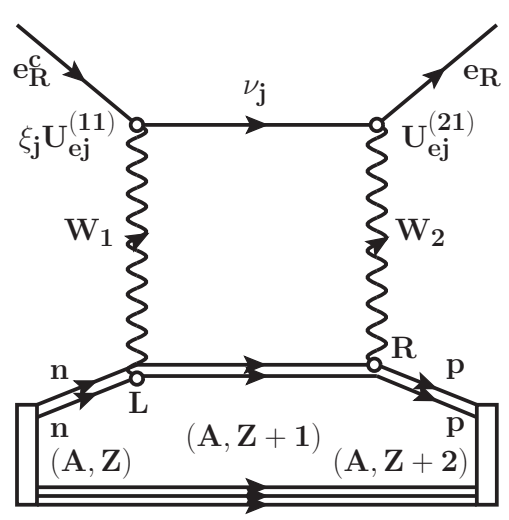

(b)

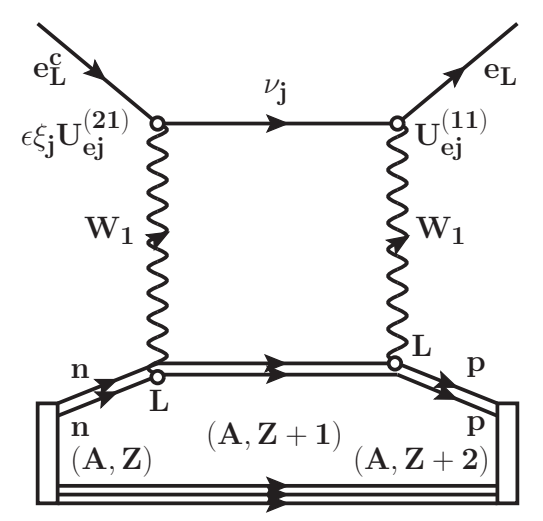

(d)

Figure 10: The Feynman diagrams at the nucleon level when the leptonic currents are of opposite chirality leading to the dimensionless lepton number violating parameters $\langle\lambda\rangle$ ( panels (a) and (b) of the figure) and $\langle\eta\rangle$ (panels (c) and (d) of the figure) of $0 \nu \beta \beta$-decay. Note that in part (a) the process proceeds via the right handed vector boson, while in part (b) through the mixing of the left and right handed bosons.

The above expression for the lifetime is now modified to yield [45, 190]:

$$
\begin{aligned}
& {\left[T_{1 / 2}^{0 \nu}\right]^{-1}=G_{01}^{0 \nu}\left|M_{G T}^{0 \nu}\right|^{2}\left\{\left|X_{L}\right|^{2}+\left|X_{R}\right|^{2}-\tilde{C}_{1}^{\prime} X_{L} X_{R}+\ldots\right.} \\
& +\tilde{C}_{2}|\langle\lambda\rangle| X_{L} \cos \psi_{1}+\tilde{C}_{3}|\langle\eta\rangle| X_{L} \cos \psi_{2}+\tilde{C}_{4}|\langle\lambda\rangle|^{2} \\
& +\tilde{C}_{5}|\langle\eta\rangle|^{2}+\tilde{C}_{6}|\langle\lambda\rangle||\langle\eta\rangle| \cos \left(\psi_{1}-\psi_{2}\right)+\operatorname{Re}\left(\tilde{C}_{2}\langle\lambda\rangle X_{R}\right. \\
& \left.\left.+\tilde{C}_{3}\langle\eta\rangle X_{R}\right)\right\}
\end{aligned}
$$

where $X_{L}$ and $X_{R}$ are defined in Eq. (65), $\psi_{1}$ and $\psi_{2}$ are the relative phases between $X_{L}$ and $\lambda$ and $X_{L}$ and $\eta$ respectively. The coefficient $\tilde{C}_{1}^{\prime}$, representing the mixing between the left and the right handed currents is kinematically suppressed[191]. The ellipses $\{\ldots\}$ indicate contributions arising from other particles, e.g., intermediate SUSY particles or unusual particles which are predicted by superstring models or exotic Higgs scalars etc 
(see below section [6).

Many nuclear matrix elements appear in this case, but they are fairly well known and they are not going to be reviewed here in detail (see e.g. 444, 62 and 663, 64, 65, 66, ). For the reader's convenience we are only going to briefly indicate them in our notation [87]) the additional nuclear matrix elements, not encountered in the mass mechanism. These are: $\chi_{F \omega}, \chi_{G T \omega}, \chi_{R}, \chi_{1^{ \pm}}, \chi_{2^{ \pm}} \chi_{F}^{\prime}, \chi_{G T}^{\prime}, \chi_{T}^{\prime}, \chi_{P}^{\prime}$ where

$$
\begin{aligned}
& \chi_{F \omega}=\left(\frac{g_{V}}{g_{A}}\right)^{2} \frac{M_{F \omega}}{M_{G T}}, \\
& \chi_{G T \omega}=\frac{M_{G T \omega}}{M_{G T}}, \\
& \chi_{R}=\frac{M_{R}}{M_{G T}}
\end{aligned}
$$

and

$$
\begin{aligned}
& \chi_{1^{ \pm}}= \pm 3 \chi_{F}^{\prime}+\chi_{G T}^{\prime}-6 \chi_{T}^{\prime}, \\
& \chi_{2^{ \pm}}= \pm \chi_{F \omega}+\chi_{G T \omega}-\frac{1}{9} \chi_{1^{ \pm}}
\end{aligned}
$$

where 87] $\left(\chi_{F}^{\prime}=M_{F}^{\prime} / M_{G T}\right.$ etc for the space part $)$ and $\left(\chi_{F \omega}=M_{F \omega} / M_{G T}\right.$ etc for the time component). In the limit in which the average energy denominator can be neglected [87], we obtain

$$
\begin{aligned}
& \chi_{F}=\chi_{F}^{\prime}=\chi_{F \omega}, \\
& \chi_{G T}=\chi_{G T}^{\prime}=\chi_{G T \omega}=1 .
\end{aligned}
$$

The quantities $G_{01}^{0 \nu}$ have been tabulated [53, [51, 63, 87, 192], see also [65] for a recent review. The coefficients $\tilde{C}_{1}^{\prime}, \tilde{C}_{i}, i=2-6$ are combinations of kinematical functions and the nuclear matrix elements have been previously discussed [192]. The coefficients $\tilde{C}_{i}, i=2, \ldots, 6$ with and without p-n pairing can be found in the literature [87]. For a more conventional formulation, restricted, however, in the light neutrino sector, see [65].

It is worth mentioning that in the case of the $\eta$, in addition to the usual Fermi Gamow-Teller and tensor terms, we have additional contributions coming from the nucleon recoil term $\left(\chi_{R}\right)$ and the kinematically favored spin antisymmetric term $\left(\chi_{P}\right)$. Due to these two effects the limit extracted for $\eta$ is much smaller than that for $\lambda$ [87]. Effective operators of a similar structure also appear in the context of R-parity violating interactions when a neutrino appears in the intermediate states (see below).

There seem to be significant changes in the nuclear matrix elements, when the $p-n$ pairing is incorporated. This point needs special care and further exploration is necessary. It has only been examined in some exactly soluble models, e.g. $\mathrm{SO}(8)$, or better approximation schemes [193, 194], but only in connection with the $2 \nu \beta \beta$-decay, or shell model calculations but for systems, which do not double beta decay [195].

Returning back to the question of the availability of nuclear matrix elements relevant for neutrinoless double beta decay, we refer once again to existing excellent recent reviews [65, 66, 192]. These reviews also provide a more detailed description of the nuclear models employed. 


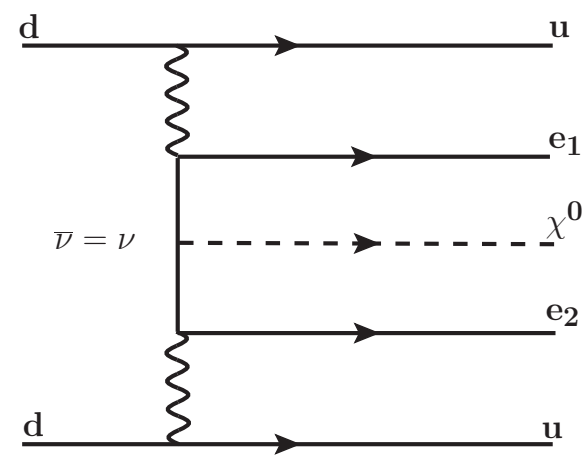

Figure 11: The Feynman diagrams at quark level leading to majoron emission in the $0 \nu \beta \beta$-decay instead of the more well known mass term. Here $\chi^{0}$ stands for the majoron, not to be confused with the neutralino, which we will encounter later in connection with supersymmetry.

\subsection{Another neutrino mass independent mechanism (majoron emission)}

It is well known that in some theories lepton number is associated with a global, not a local, symmetry. When such theories are broken spontaneously, one encounters physical Nambu-Goldstone bosons, called majorons. These bosons only couple to the neutrinos. So in any model which gives rise to mass term for the light neutrino (mass insertion in the neutrino propagator), one may naturally have a competing majoron-neutrinoantineutrino coupling. Such a mechanism is shown at the quark level in Fig. 11. The majoron, which couples to the left handed neutrinos, comes from the neutral member of the isotriplet. Such a multiplet, however, cannot easily be be accommodated theoretically. So this type of majoron is not present in the usual models. On the other hand there is a majoron $\chi^{0}$, the imaginary part of an isosinglet scalar, which couples to the right handed neutrino with a coupling $g_{i j}^{0}$. This gives rise to the mechanism shown in Fig. 12 at the nucleon level. The right handed neutrino, however, has a small component of light neutrinos (see Eq. (27) ).

$$
\mathcal{L}_{\nu \nu \chi^{0}}=\sum_{i<j} g_{i j}\left[\bar{\nu}_{i L} \gamma_{5} \nu_{j L}\right] \chi^{0}
$$

with

$$
g_{i j}=\sum_{i<j} U_{\alpha i}^{(21)} U_{\beta j}^{(21)} g_{\alpha \beta}^{0} \xi_{i}
$$

with $g_{\alpha \beta}^{0}$ the coupling of the majoron to the corresponding neutrino flavors. The expression for the half-life takes the form

$$
\left[T_{1 / 2}^{0 \nu}\right]^{-1}=G_{01}^{\chi}\left|\langle g\rangle M_{\nu}^{0 \nu}\right|^{2}
$$

with $\langle g\rangle=\sum_{i<j} U_{e i}^{(21)} U_{e j}^{(21)} g_{i j}$. Notice that, even if $g_{i j}$ takes natural values, the coupling $g_{i j}$ is very small due to the smallness of the mixing matrix $U^{(21)}$. Thus the effective coupling $\langle g\rangle$ is very small. So, even though we do not suffer in this case from the 


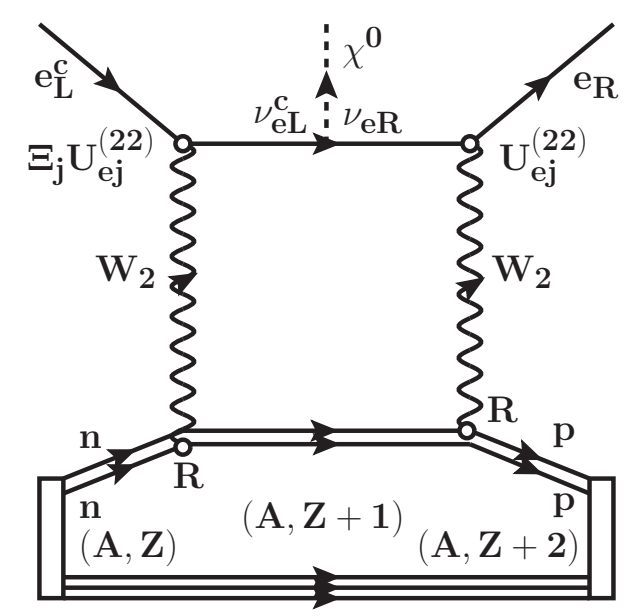

Figure 12: The same process as in Fig 11, but written at the nucleon level in the case of the isosinglet majoron, which couples to the right handed neutrinos.

suppression due to the smallness of the mass of the neutrino, the majoron emission mechanism is perhaps unobservable. There exist, however, exotic models, which, in principle, may allow majoron emission with a large coupling, like the bulk majoron [196] and others [197, 198], which we are not going to pursue this theoretically any further. In any case it is straightforward to extract the limits on the effective coupling $\langle g\rangle$, since the nuclear matrix elements are the same as in the light neutrino mass mechanism. Note, however, that the spectrum of the summed energy of the two electrons is continuous and the kinematical function is different.

\section{Mechanisms in $0 \nu \beta \beta$-decay not involving intermediate neutrinos}

\subsection{The direct decay of doubly charged particles to leptons}

Such a candidate is the isotriplet scalar, which can generate Majorana neutrino mass as see-saw mechanism II. The doubly charged component can directly decay into two leptons (see Fig. 13). The coupling to the quarks is achieved via the charged Higgs isodoublet in models where it survives the Higgs mechanism (e.g. SUSY) or through gauge bosons (Figs 13a and 13b respectively). For consistency between the two we

slightly change the notation here implying that the cubic coupling $\mu_{\Delta}$ originates from a dimensionless quartic coupling $\lambda$ of the Higgs particles of the figure with an isosinqlet scalar acquiring a vacuum expectation value $v_{R}$.

The lepton number violating parameter associated with Fig. 13 is given by:

$$
\eta_{\Delta H}=\frac{2 \sqrt{2} \lambda v_{R} m_{u}^{2} m_{p} g_{e e}}{m_{H}^{4} m_{\Delta_{L}}^{2} G_{F}}, \quad \eta_{\Delta W}=\frac{v_{R} m_{W}^{2} m_{p} g_{e e}}{m_{W_{R}}^{4} m_{\Delta_{R}}^{2} 4 G_{F}}
$$

Taking $g_{e e}=1$, a natural value, and $\lambda v_{R} \approx 1 \mathrm{TeV}, m_{H}=m_{\Delta}=100 \mathrm{GeV}$ and $m_{u}=10$ $\mathrm{MeV}$, one finds [4] $\eta_{\Delta H} \approx 3 \times 10^{-8}$. Similarly taking $v_{R}=m_{W_{R}}=m_{\Delta_{R}}=m_{W} / \sqrt{\kappa}=$ 


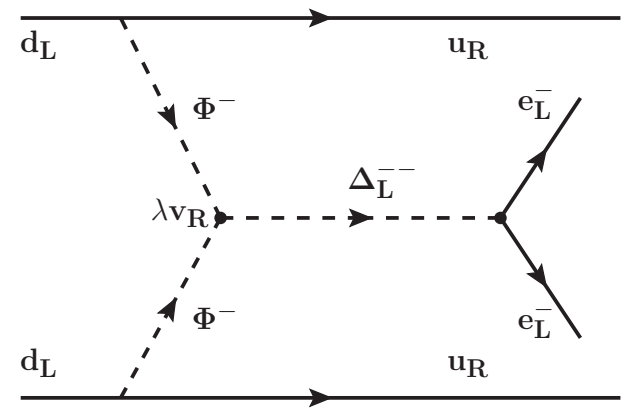

(a)

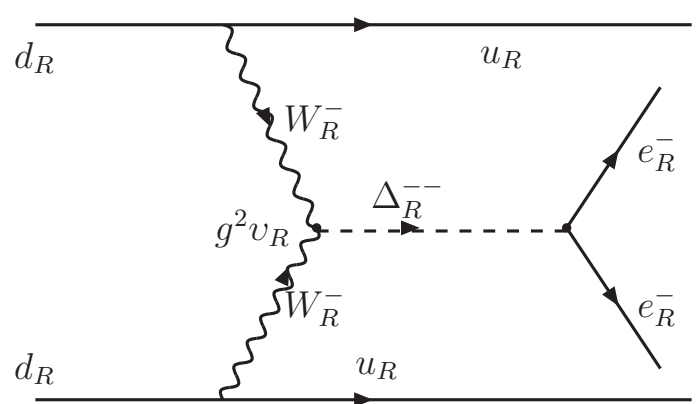

(b)

Figure 13: We show the direct decay of the isotriplet into two leptons (a). The coupling to the quarks is achieved via the charged Higgs isodoublet in models where it survives the Higgs mechanism (e.g. SUSY). In (b) we show the direct decay into two leptons of an isosinglet doubly charged Higgs, present in left-right symmetric models. The coupling to the quarks is now achieved via the right handed gauge bosons.

$10 m_{W}$, we find $\eta_{\Delta W} \approx 10^{-6}$.

Note that the term $\eta_{\Delta H}$ adds to $X_{L}$ of Eq. (64), while $\eta_{\Delta W}$ adds to the $X_{R}$.

\subsection{The R-parity violating contribution to $0 \nu \beta \beta$ decay.}

In SUSY theories R-parity is defined as

$$
R=(-1)^{3 B+L+2 s},
$$

with $B=$ baryon, $L=$ lepton numbers and s the spin. It is +1 for ordinary particles and -1 for their superpartners. R-parity violation has recently been seriously considered in SUSY models. It allows additional terms in the superpotential given by

$$
W=\epsilon_{i} L_{i}^{a} H_{2 a}+\lambda_{i j k} L_{i}^{a} L_{j}^{b} E_{k}^{c} \epsilon_{a b}+\lambda_{i j k}^{\prime} L_{i}^{a} U_{j}^{b} D_{k}^{c} \epsilon_{a b}+\lambda_{i j k}^{\prime \prime} U_{i}^{c} U_{j}^{c} D_{k}^{c},
$$

where a summation over the flavor indices $i, j, k$ and the isospin indices a,b is understood $\left(\lambda_{i j k}\right.$ is antisymmetric in the indices $i$ and $j$ ). The last term has no bearing in our discussion, but we will assume that it vanishes due to some discreet symmetry to avoid too fast proton decay. The first term is a lepton number violating bilinear and, since it cannot be rotated away, it can lead to neutrinoless double beta decay. The $\lambda$ 's are dimensionless couplings not predicted by the theory. The couplings are assumed to be given in the basis in which the charged fermions are diagonal. In the above notation $\mathrm{L}, \mathrm{Q}$ are isodoublet and $E^{c}, D^{c}$ isosinglet chiral superfields, i.e they represent both the fermion and the scalar components.

The above R-parity violating superpotential can lead to Majorana neutrino masses without the need of introducing the right-handed neutrino and invoking the see-saw mechanism [95, 142]. One then can have contributions to neutrinoless double beta decay in the usual way via intermediate massive neutrinos as discussed above. 


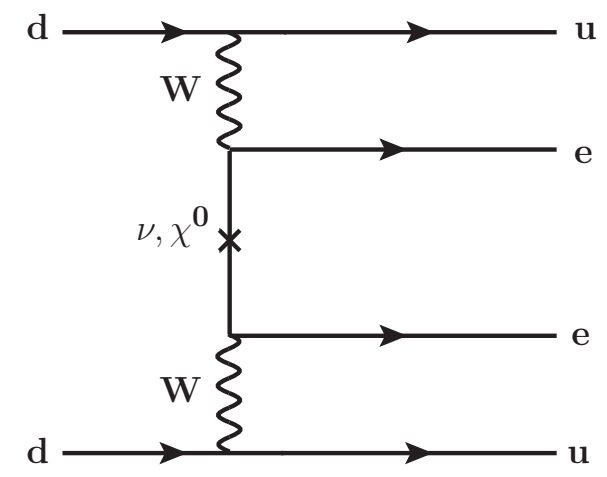

Figure 14: The R-parity violating contribution to $0 \nu \beta \beta$ decay mediated by neutralinos arising from the bilinear terms in the superpotential. For comparison we give the neutrino mediated process of Fig. 8 expressed at the quark level.

\subsubsection{The contribution arising from the bilinears in the superpotential.} The first term in the superpotential, Eq. (82), can cause mixing between the neutrino and the neutralinos as soon as the s-neutrino develops a vacuum expectation value. As a result it can directly lead to neutrinoless double beta decay [94, 95, 199] via the effective W-charged lepton-neutralino interaction:

$$
\mathcal{L}=-\frac{g}{\sqrt{2}} \kappa_{n} W_{\mu}^{-} \bar{e}_{L} \gamma^{\mu} \chi_{n L}^{0} .
$$

where $\kappa_{n}$ is a dimensionless quantity, associated with each of the four neutralinos, which arises due to neutrino neutralino mixing [94]. This term then gives rise to a diagram analogous to that of Fig. 8 with the intermediate particle now being the neutralino, which is heavy with mass $M_{\chi_{n}^{0}}$ and leads to a short ranged operator. For the reader's convenience this is shown in Fig. 14 at the quark level. One thus obtains an analogous lepton number violating parameter:

$$
\eta_{N}^{L} \rightarrow \eta_{\chi^{0}}^{L}=\sum_{n} \kappa_{n}^{2} \frac{m_{p}}{M_{\chi_{n}^{0}}} .
$$

6.2.2. The contribution arising from the cubic terms in the superpotential. It has also been recognized quite sometime ago that the second and third terms (cubic terms) in the superpotential could lead to neutrinoless double beta decay [26, 88, 89]. Typical diagrams at the quark level are shown in Fig. 15, Note that as intermediate states, in addition to the sleptons and squarks, one must consider the neutralinos, 4 states which are linear combinations of the gauginos and higgsinos, and the colored gluinos (supersymmetric partners of the gluons). Whenever the process is mediated by gluons a Fierz transformation is needed to lead to a colorless combination. The same thing is necessary whenever the fermion line connects a quark to a lepton. As a result one gets at the quark level not only scalar (S) and pseudoscalar (P) couplings, but tensor $(\mathrm{T})$ couplings as well. This must be contrasted to the $\mathrm{V}$ and A structure of the traditional 

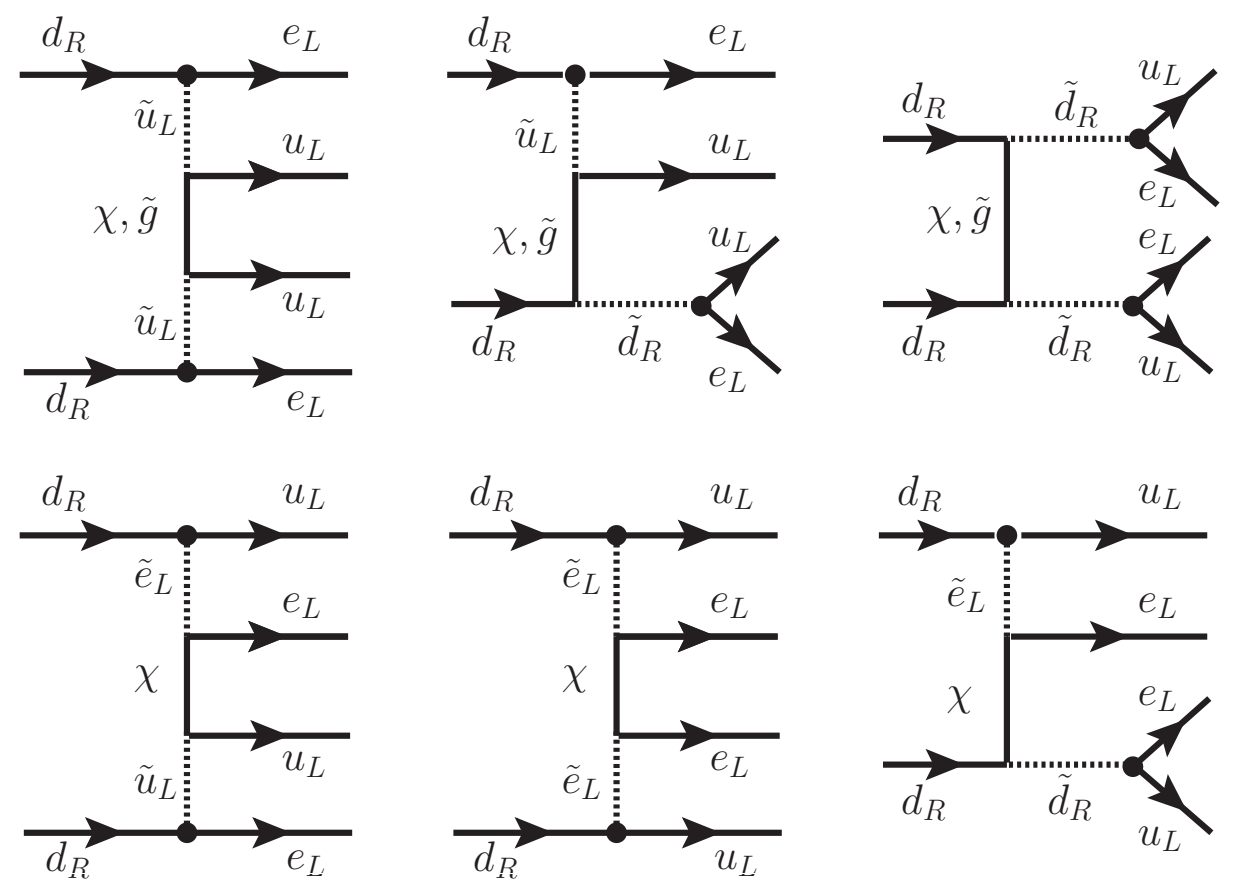

Figure 15: The R-parity violating contribution to $0 \nu \beta \beta$ decay mediated by sfermions and neutralinos (gluinos) arising from the cubic terms in the superpotential. The dots indicate the lepton violating R-parity interactions.

mechanisms. One, therefore, must face the problem of how to transform these operators from the quark to the nucleon level.

\subsubsection{The lepton number violating parameters from the cubic terms without intermediate} neutrinos. As we have mentioned the effective lepton number violating parameter arising from the bilinear terms in the superpotential is analogous to that arising from the heavy intermediate neutrinos and, thus, it will not be further discussed. We will concentrate on the cubic terms in the superpotential [26, 66, 200, 201, 202]. Then the effective lepton number violating parameter arising from these terms, assuming that the pion exchange mode dominates, as the authors of Refs. [26, 66] find, can be written as

$$
\eta_{S U S Y}=\left(\lambda_{111}^{\prime}\right)^{2} \frac{3}{8}\left(\chi_{P S} \eta_{P S}+\eta_{T}\right)
$$

with $\eta_{P S}\left(\eta_{T}\right)$ associated with the scalar and pseudoscalar (tensor) quark couplings given by

$$
\begin{aligned}
& \eta_{P S}=\eta_{\tilde{\chi}, \tilde{e}}+\eta_{\tilde{\chi}, \tilde{q}}+\eta_{\tilde{\chi}, \tilde{f}}+\tilde{\eta}_{\tilde{g}}+7 \eta_{\tilde{g}}^{\prime} \\
& \eta_{T}=\eta_{\tilde{\chi}, \tilde{q}}-\eta_{\tilde{\chi}, \tilde{f}}+\tilde{\eta}_{\tilde{g}}-\eta_{\tilde{g}}^{\prime}
\end{aligned}
$$

These authors find $\chi_{P S}=(5 / 3)$, but, as we shall see below, it depends, in general, on ratios of nuclear matrix elements. For the diagram of Fig. 15a one finds

$$
\eta_{\tilde{\chi}, \tilde{e}}=\frac{2 \pi \alpha}{\left(G_{F} m_{W}^{2}\right)^{2}}\left(\kappa_{\tilde{e}}\right)^{2}\left\langle\frac{m_{p}}{m_{\tilde{\chi}}}\right\rangle_{\tilde{e} \tilde{e}} \text {. }
$$


For the diagram of Fig. 15b one finds

$$
\begin{aligned}
& \tilde{\eta}_{\tilde{\chi}, \tilde{q}}=\frac{\pi \alpha}{2\left(G_{F} m_{W}^{2}\right)^{2}}\left[\left(\kappa_{\tilde{d}}\right)^{2}\left\langle\frac{m_{p}}{m_{\tilde{\chi}}}\right\rangle_{\tilde{d} \tilde{d}}+\left(\kappa_{\tilde{u}}\right)^{2}\left\langle\frac{m_{p}}{m_{\tilde{\chi}}}\right\rangle_{\tilde{u} \tilde{u}}\right], \\
& \tilde{\eta}_{\tilde{g}}=\frac{\pi}{6} \alpha_{s} \frac{1}{\left(G_{F} m_{W}^{2}\right)^{2}}\left[\left(\kappa_{\tilde{d}}\right)^{2}+\left(\kappa_{\tilde{u}}\right)^{2}\right] \frac{m_{p}}{m_{\tilde{g}}} .
\end{aligned}
$$

For the diagram of Fig. 15k one finds

$$
\begin{aligned}
& \tilde{\eta}_{\tilde{\chi}, \tilde{f}}=\frac{\pi \alpha}{2\left(G_{F} m_{W}^{2}\right)^{2}}\left[\kappa_{\tilde{e}} \kappa_{\tilde{d}}\left\langle\frac{m_{p}}{m_{\tilde{\chi}}}\right\rangle_{\tilde{e} \tilde{d}}+\kappa_{\tilde{e}} \kappa_{\tilde{u}}\left\langle\frac{m_{p}}{m_{\tilde{\chi}}}\right\rangle_{\tilde{e} \tilde{u}}+\kappa_{\tilde{d}} \kappa_{\tilde{u}}\left\langle\frac{m_{p}}{m_{\tilde{\chi}}}\right\rangle_{\tilde{u} \tilde{u}}\right], \\
& \tilde{\eta}_{\tilde{g}^{\prime}}=\frac{\pi}{12} \alpha_{s} \frac{1}{\left(G_{F} m_{W}^{2}\right)^{2}} \kappa_{\tilde{d}} \kappa_{\tilde{u}} \frac{m_{p}}{m_{\tilde{g}}},
\end{aligned}
$$

where

$$
\begin{aligned}
& \kappa_{X}=\left(\frac{m_{W}}{m_{X}}\right)^{2}, X=\tilde{e}_{L}, \tilde{u}_{L}, \quad \kappa_{\tilde{d}}=\left(\frac{m_{W}}{m_{\tilde{d}_{R}}}\right)^{2} . \\
& \left\langle\frac{m_{p}}{m_{\tilde{\chi}}}\right\rangle_{\tilde{f} \tilde{f}^{\prime}}=\sum_{i=1}^{4} \epsilon_{\tilde{\chi}_{i}, \tilde{f}^{\prime}} \epsilon_{\tilde{\chi}_{i}, \tilde{f}^{\prime}} \frac{m_{p}}{m_{\tilde{\chi}_{i}}},
\end{aligned}
$$

where $\epsilon_{\tilde{\chi}_{i}, \tilde{f}}$ and $\epsilon_{\tilde{\chi}_{i}, \tilde{f}^{\prime}}$ are the couplings of the $i^{\text {th }}$ neutralino to the relevant fermion and sfermion. These are calculable (see, e.g., Ref. [44]). Thus ignoring the small Yukawa couplings coming via the Higgsinos and taking into account only the gauge couplings, we find

$$
\begin{aligned}
\epsilon_{\tilde{\chi}_{i}, \tilde{e}} & =\frac{Z_{2 i}+\tan \theta_{W} Z_{1 i}}{\sin \theta_{W}}, \\
\epsilon_{\tilde{\chi}_{i}, \tilde{u}} & =\frac{Z_{2 i}+\left(\tan \theta_{W} / 3\right) Z_{1 i}}{\sin \theta_{W}}, \\
\epsilon_{\tilde{\chi}_{i}, \tilde{d}} & =-\frac{Z_{1 i}}{3 \cos \theta_{W}},
\end{aligned}
$$

where $Z_{1 i}, Z_{2 i}$ are the coefficients in the expansion of the $\tilde{B}, \tilde{W}_{3}$ in terms of the neutralino mass eigenstates. Note that in this convention some of the masses $m_{\tilde{\chi}_{i}}$ may be negative.

We should mention here that, if the gluino exchange is dominant, the lepton number violating parameter $\eta_{\mathrm{SUSY}}$ simplifies and becomes $\eta_{\lambda^{\prime}}$ with $\eta_{\lambda^{\prime}}$ given in section 9 .

6.2.4. The case of light intermediate neutrinos It is also possible to have light neutrino mediated $0 \nu \beta \beta$ decay originating from $\mathrm{R}$-parity violating interactions. In this case one has the usual $\beta$ decay vertex of the $V-A$ type in one vertex and the sfermion mediated vertex, of the $S-P$ type, in the other end [95, 199, 200] (see Fig. 16)). The lepton number violation is achieved via the mixing of isodoublet and isosinglet sfermions. The simplest diagram, which involves intermediate sleptons, can arise from the following interactions:

$$
\mathcal{L}_{L Q D^{c}} \rightarrow \lambda_{111}^{\prime} \sum_{k} V_{e k}^{* L} \bar{u}_{L} d_{R} \tilde{\ell}_{k}^{*}
$$




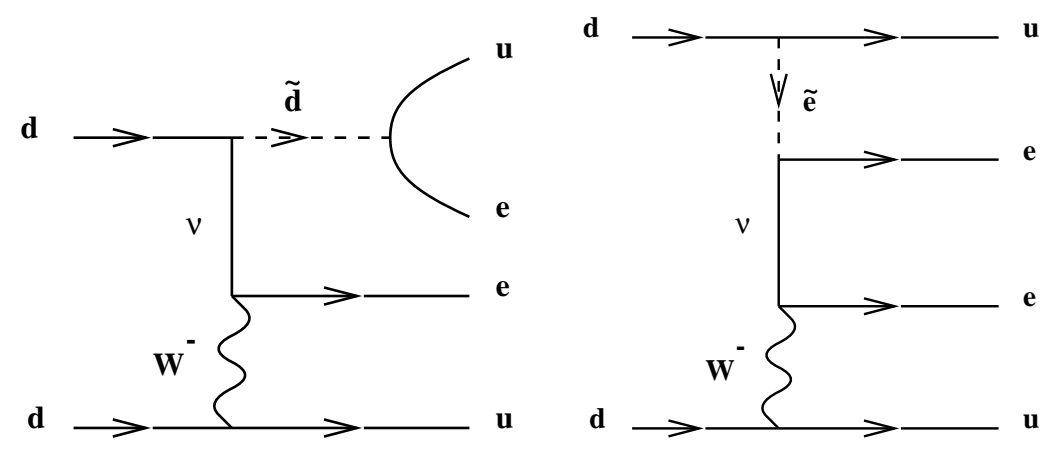

Figure 16: The light neutrino mediated $0 \nu \beta \beta$-decay in R-parity violating SUSY models. In addition to the usual gauge vertex one has a scalar vertex mediated by sleptons or down-type squarks. The lepton number violation proceeds via the mixing between the isodoublet and isosinglet sfermions. This is not indicated by an $\times$ on the scalar line, as it is customary, since we use the sfermion mass eigenstates.

for the d-quark vertex and

$$
\mathcal{L}_{L L E^{c}} \rightarrow \lambda_{111} \sum_{j, k} V_{e k}^{R} U_{e j} \bar{\nu}_{j L} e_{R}^{c} \tilde{\ell}_{k}
$$

for the neutrino vertex. In the above expression $V^{L}\left(V^{R}\right)$ are the mixing matrices which express the doublet (singlet) selectrons in terms of the mass eigenstates. $U$ is the usual neutrino mixing matrix. The effective s-lepton propagator is

$$
P=\sum_{k=1}^{3} \frac{V_{e k}^{L} V_{e k}^{R}}{M_{k}^{2}} \approx \sum_{k=1}^{2} V_{e k}^{L} V_{e k}^{R} \frac{\delta M_{k}^{2}}{M_{e}^{4}}, \quad \delta M_{k}^{2}=M_{k}^{2}-M_{3}^{2} .
$$

The last equation follows from the orthogonality condition on the mixing matrices and the fact that the splitting $\delta M_{k}^{2}$ is small compared to the average s-lepton mass $M_{e}^{2}$. If, further, the mixing between generations can be ignored we get

$$
P=\frac{\sin 2 \theta_{\tilde{e}}}{2} \frac{\Delta M_{e}^{2}}{M_{\tilde{e}}^{4}}
$$

Combining the above results with the usual $V-A$ coupling one gets:

$$
\mathcal{M}=\left(\frac{G_{F}}{\sqrt{2}}\right)^{2} \Lambda_{\tilde{e}} \bar{u}_{L} d_{R} \bar{e}_{L} \frac{k_{\alpha} \gamma^{\alpha}}{k^{2}} \gamma^{\lambda} e_{R}^{c} \bar{u}_{L} \gamma_{\lambda} d_{L}
$$

with

$$
\Lambda_{\tilde{e}}=\frac{\sqrt{2}}{G_{F}} \lambda_{111}^{\prime} \lambda_{111} \sum_{j} U_{e j}^{2} \frac{\sin 2 \theta_{\tilde{e}}}{2} \frac{\Delta M_{e}^{2}}{M_{\tilde{e}}^{4}}
$$

In the case of squark exchange of Fig. 16 the above expressions become

$$
\mathcal{L}_{L Q D^{c}} \rightarrow \lambda_{111}^{\prime} \sum_{k} V_{d k}^{* L} \bar{u}_{L} e_{R}^{c} \tilde{d}_{k}^{*}
$$

for the u-quark vertex and

$$
\mathcal{L}_{L Q D^{c}} \rightarrow \lambda_{111}^{\prime} \sum_{k} V_{d k}^{R} U_{e j} \bar{\nu}_{j L} d_{R} \tilde{d}_{k}
$$


for the neutrino vertex. Combining them we get

$$
\mathcal{M}=\left(\frac{G_{F}}{\sqrt{2}}\right)^{2} \Lambda_{\tilde{d}} \bar{u}_{L} e_{R}^{c} \bar{e}_{L} \gamma^{\lambda} \frac{k_{\alpha} \gamma^{\alpha}}{k^{2}} d_{R}, \bar{u}_{L} \gamma_{\lambda} d_{L}
$$

with

$$
\Lambda_{\tilde{d}}=\frac{\sqrt{2}}{G_{F}}\left(\lambda_{111}^{\prime}\right)^{2} \sum_{j}\left(U_{e j}\right)^{2} \frac{\sin 2 \theta_{\tilde{d}}}{2} \frac{\Delta M_{\tilde{d}}^{2}}{M_{\tilde{d}}^{4}}
$$

in a rather obvious notation.

In the case $\tilde{e}$ and $\tilde{d}$ contributions, in the context of perturbation theory, one can simplify the above expressions using explicitly the coupling between the singlet and the doublet sfermions of the lower charge. In this case

$$
\frac{\sin 2 \theta_{\tilde{x}}}{2} \Delta M_{\tilde{x}}^{2}=(\mu+A \tan \beta) m_{x} \quad x=e, d .
$$

Before proceeding farther we have to perform a Fierz transformation:

$$
\begin{aligned}
\bar{u}_{L} e_{R}^{c} \bar{e}_{L} \gamma^{\lambda} k_{\alpha} \gamma^{\alpha} d_{R} & =-\frac{1}{2}\left[\bar{u}_{L} d_{R} \bar{e}_{L} e_{R}^{c} k_{\lambda}+\bar{u}_{L} d_{R} \bar{e}_{L} i \sigma_{\lambda \nu} k^{\nu} e_{R}^{c}\right. \\
& \left.+\bar{u}_{L} i \sigma_{\lambda \nu} k^{\nu} d_{R} \bar{e}_{L} e_{R}^{c}\right]-\frac{1}{8} \bar{u}_{L} i \sigma_{\alpha \beta} d_{R} \bar{e}_{L} i \sigma^{\alpha \beta} e_{R}^{c} k_{\lambda} .
\end{aligned}
$$

We must now go to the nucleon level and perform a Fourier transform to the coordinate space. For $0^{+} \rightarrow 0^{+}$transitions the space component yields:

$$
\begin{aligned}
\mathcal{M} & =\left(\frac{G_{F}}{\sqrt{2}}\right)^{2}\left(f_{A}\right)^{2}\left(-\lambda\left[M_{T}^{\prime}+M_{G T}^{\prime}+r_{F} M_{F}^{\prime}\right] \bar{e} \gamma_{0} \mathbf{q} \cdot \gamma\left(1+\gamma_{5}\right) e^{c}\right) \\
& -\frac{\Lambda_{d}}{2}\left(\tilde{M}_{G T}+\left(1-2 \frac{\Lambda_{e}}{\Lambda_{d}}\right) \frac{1}{f_{A}^{2}} \tilde{M}_{F}\right) q_{0} \bar{e}\left(1+\gamma_{5}\right) e^{c}
\end{aligned}
$$

with

$$
\lambda=\Lambda_{\tilde{d}} / 96, \quad r_{F}=\frac{3}{4 f_{A}^{2}}\left(-2 \frac{\Lambda_{\tilde{e}}}{\Lambda_{\tilde{d}}}+1\right) .
$$

The parameter $\lambda$ as well as the quantities $M^{\prime}$ and $\tilde{M}$ have the same meaning as in the mass independent contribution in the conventional approach (see section 5.2). Note, however, that in the present mechanism there is no term analogous to the $\eta$ of section 5.2. We should stress that this novel mechanism can lead to transitions $J^{+}, J \neq 0$. So, contrary to conventional wisdom, from the observation of such transitions one cannot definitely infer the existence of right handed currents.

\section{Handling the short range transition operators}

We have seen that there exist many mechanisms contributing to neutrinoless double beta decay, involving the exchange of only heavy particles. These result to short range transition operators. 


\subsection{The mode involving only nucleons}

If the nucleons are treated as point like particles, then the effective transition operator essentially behaves like a $\delta$ function in the inter nucleon distance. Thus their contribution vanishes, due to the presence of a nuclear hard core. This can be cured, if the nucleons can be treated as extended objects. This can be done by introducing into the nucleon current a nucleon form factor [96], e.g. like a dipole shape with a characteristic mass $m_{A} \approx 850 \mathrm{MeV}$. This can also be accomplished, if one utilizes a quark model for the nucleon [203].

- V-A theories

In this case the approach has become pretty standard, i.e. the spin isospin structure is similar to that for the light neutrino mass term except for the radial part [44], which now becomes:

$$
\frac{m_{A}^{2}}{m_{e} m_{p}} F_{N}\left(x_{A}\right) \frac{R_{0}}{r_{k \ell}}, \quad x_{A}=r_{k \ell} m_{A}, \quad F_{N}(x)=\frac{x}{48}\left(x^{2}+3 x+3\right) e^{-x}
$$

and we are not going to discuss them further. We only mention that it can also proceed via the 2-pion mode with the nuclear operator associated with $\alpha_{2 \pi}=0.1$ (see next section)

- $S, P S$ theories

In the scalar case we find that the operator is spin independent and has the same radial part as in the previous case.

In the case of the pseudo scalar part we find that the operator becomes

$$
\begin{aligned}
& \Omega_{P S}=\frac{m_{A}^{2}}{m_{e} m_{p}} \frac{1}{3}\left(\frac{m_{A}}{2 m_{p} / 3}\right)^{2} \sum_{k \neq \ell} \tau_{+}(k) \tau_{+}(\ell) \times \\
& \left(\sigma_{k} \cdot \sigma_{\ell} \frac{2}{x_{A}} \frac{d F_{N}\left(x_{A}\right)}{d x_{A}}+T\left(\hat{x}_{A}, \sigma_{k}, \sigma_{\ell}\right)\left(-\frac{d F_{N}\left(x_{A}\right)}{x_{A} d x_{A}}+\frac{d F_{N}^{2}\left(x_{A}\right)}{d x_{A}^{2}}\right)\right)
\end{aligned}
$$

where $T\left(\hat{x}_{A}, \sigma_{k}, \sigma_{\ell}\right)$ is the tensor component.

This process can also proceed via the 2-pion mode (see next section). In this case one can use the lepton number violating parameter $\eta_{\Delta H}$ with nuclear matrix element as given in the next section with $\alpha_{2 \pi}=0.20$.

\subsection{The pion mode in $R$-parity induced $0 \nu \beta \beta$ decay.}

Even though the pion model, (12), may be important in other cases when the intermediate particles are heavy, giving rise to short range operators, in this section we will elaborate a bit further on its application in the extraction of the R-parity violating parameters associated with the processes discussed above. The nuclear matrix elements can now be calculated using the effective transition operators

$$
M E_{k}=\left(\frac{m_{A}}{m_{p}}\right)^{2} \alpha_{k \pi} \frac{m_{p}}{m_{e}}\left[M_{G T}^{k \pi}+M_{T}^{k \pi}\right] .
$$



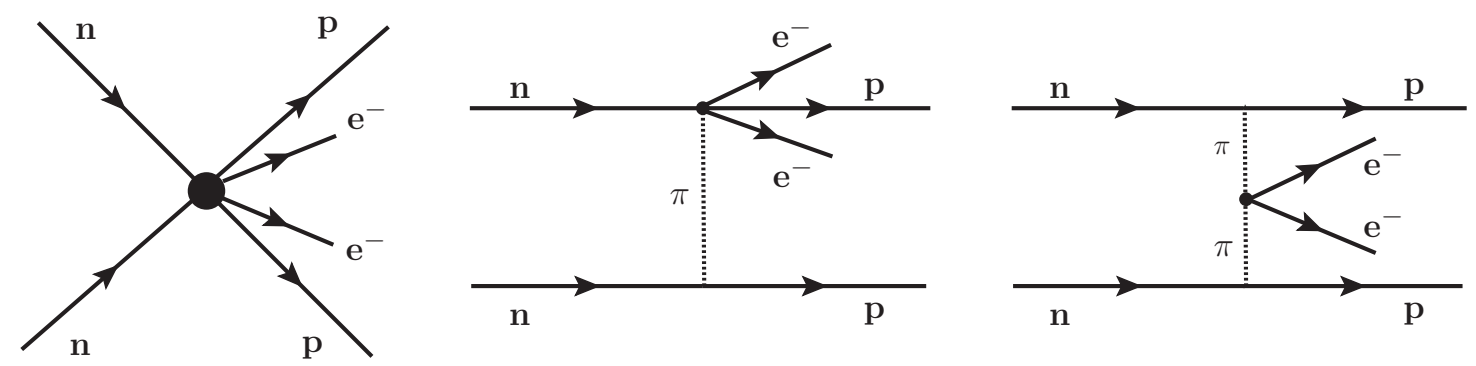

Figure 17: The pion mediated $0 \nu \beta \beta$-decay as a contact interaction (a). It arises as an $1 \pi$, (b) (an analogous $\pi$-vertex in the other nucleon is understood), and $2 \pi$ exchange contributions (c). The lepton number violation occurs either in one nucleon (b) or in the pions $(\mathrm{c})$.

Where the two above matrix elements are the usual GT and T matrix elements with the additional radial dependence given by

$$
\begin{aligned}
& F_{G T}^{1 \pi}=e^{-x}, \quad F_{T}^{1 \pi}=\left(3+3 x+x^{2}\right) \frac{e^{-x}}{x}, \\
& F_{G T}^{2 \pi}=(x-2) e^{-x}, \quad F_{T}^{2 \pi}=(1+x) e^{-x},
\end{aligned}
$$

In the case of the pion exchange mechanism, in particular for $1 \pi$ exchange, it is important to include the nucleon form factors [202, 204]. We are not going to elaborate further on this point. The complete expressions for the transition operators are given in section 9 ,

We will, instead, concentrate on the coefficients $\alpha_{2 \pi}$ and $\alpha_{1 \pi}$. We will begin by considering the elementary particle treatment [27].

- The coupling coefficients $\alpha_{2 \pi}$. One finds

$$
\alpha_{2 \pi}=\frac{1}{6 f_{A}^{2}} g_{r}^{2} h_{\pi}^{2}\left(\frac{m_{\pi}}{m_{p}}\right)^{4}
$$

Obtained under the factorization approximation[27]:

$$
\begin{aligned}
<\pi^{+}\left|J_{P} J_{P}\right| \pi^{-}> & =\frac{5}{3}<\pi^{+}\left|J_{P}\right| 0><0\left|j_{P}\right| \pi^{-}>, \\
<0\left|J_{P}\right| \pi^{-}> & =m_{\pi}^{2} h_{\pi}
\end{aligned}
$$

with the parameter $h_{\pi}$ given by

$$
h_{\pi}=0.668 \sqrt{2} i \frac{m_{\pi}}{m_{d}+m_{u}}
$$

Thus using the current quark masses these authors [27] find $\alpha_{2 \pi}=0.20$.

- The coupling coefficients $\alpha_{1 \pi}$

One finds

$$
\alpha_{1 \pi}=-F_{P} \frac{1}{36 f_{A}^{2}} g_{r} h_{\pi}\left(\frac{m_{\pi}}{m_{p}}\right)^{4}
$$


The needed parameters were obtained using the factorization approximation in the case of $1-\pi$ mode

$$
\begin{aligned}
<p\left|J_{P} J_{P}\right| n \pi> & =\frac{5}{3}<p\left|J_{P}\right| n><0\left|J_{P}\right| \pi^{-}> \\
<p\left|J_{P}\right| n> & =F_{P} \approx 4.41
\end{aligned}
$$

The matrix element $<0\left|J_{P}\right| \pi^{-}>$was given above (see Eq. (117)). Thus these authors [27] find:

$$
\alpha_{1 \pi}=-4.4 \times 10^{-2}
$$

In order to provide a check of the approximations involved in the above treatment and to explore the new possibilities appearing in the microscopic treatment, e.g. the role played by the non local terms or the three possibilities entering in Fig. 18, which are not distinguished in the standard treatment, we will consider the quark structure of the pion and the nucleon [203]. Thus we will evaluate the relevant amplitude by making a non relativistic expansion of the hadronic current employing a constituent quark mass equal to $1 / 3$ of the nucleon mass. Furthermore for the pion and the nucleon internal relative quark wave functions we will employ harmonic oscillator wave functions, adjusting the size parameters to fit related experiments [203. Then we will compare this amplitude to that obtained by more standard techniques [93].

a) The $1 \pi$ mode.

Let us begin with the second process of Eq. (12) (see diagrams (b) of Fig. 17), which is further analyzed at the quark level in Fig. 18. In this case it is clear that the amplitude must be of the PS type only. The tensor contribution cannot lead to a pseudoscalar coupling at the nucleon level. Such a coupling is needed to be combined with the usual pion nucleon coupling in the other vertex to get the relevant operator for $\mathrm{a}^{+} \rightarrow 0^{+}$decay.

Let us begin with diagram 18 (a), which is simply the decay of the pion into two leptons with a simultaneous change of a neutron to a proton by the relevant nucleon current. Then one finds that, if the non local terms, which lead to new type operators not studied up to now, are ignored, the "direct" diagram makes no contribution.

The "exchange" contribution, Fig. 18 (b), in which the produced up quark of the meson is not produced from the "vacuum" but comes from the initial nucleon, is a bit more complicated. The result is:

$$
c_{1 \pi}=1.37 f_{1 \pi}^{c o n}(x), \quad \alpha_{1 \pi}=0.071 f_{1 \pi}^{c o n}(x) \approx 0.071,
$$

which is in size almost a factor of 2 larger than that obtained in elementary particle treatment [27] (see Eq.(121) ) . Note, however, that our results depend on the pion size parameter. Finally diagram 18 (c), in which the $q \bar{q}$ is produced by the weak interaction, leads to the expression:

$$
c_{1 \pi}=\frac{20 \sqrt{2} \sqrt[4]{\pi} \sqrt{m_{\pi}}}{3 g_{r} \sqrt{b_{N}^{3}} m_{N}^{2}} f_{1 \pi}^{A}(x)=3.4 \times 10^{-2} f_{1 \pi}^{A}(x)(\text { constituent masses })(123)
$$




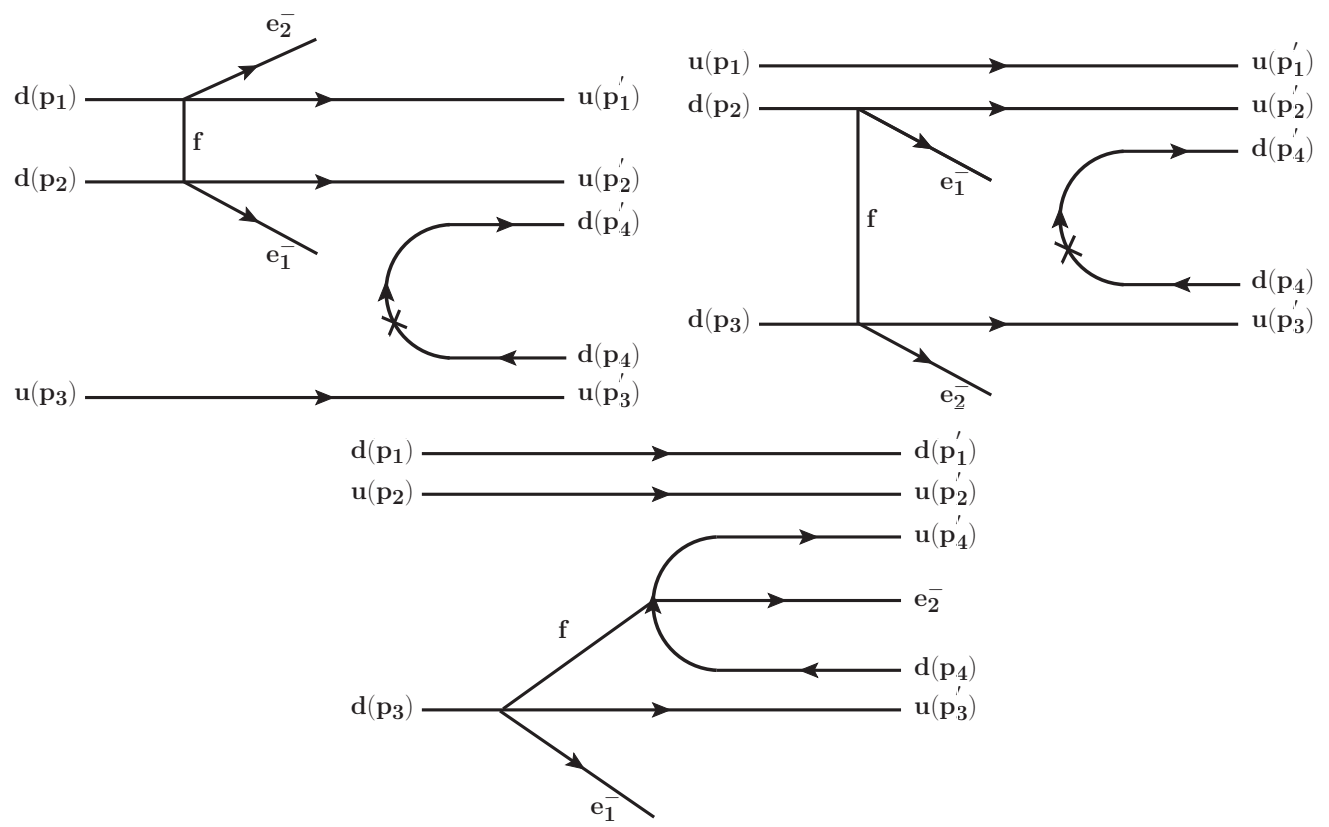

Figure 18: The pion mediated $0 \nu \beta \beta$-decay in the so-called $1 \pi$ mode. In diagram (a) the quarks of the pion are spectators, i.e. the heavy intermediate heavy fermion $\mathrm{f}$ is exchanged between the other two quarks. In (b) one of the interacting quarks is in the pion. In (c) the $q \bar{q}$ pair is produced by the weak interaction, while in (a) and (b) the $q \bar{q}$ is produced by the strong interaction out of the vacuum in the context of a multigluon exchange (a la $3 p_{0}$ mode) indicated by $\times . f$ stands for an intermediate neutral fermion (heavy Majorana neutrino, gluino or neutralino).

with

$$
f_{1 \pi}^{A}(x)=x^{3 / 2}, \quad x=\frac{b_{N}}{b_{\pi}}
$$

The corresponding coefficient that must multiply the nuclear matrix element for $x=2$ is

$$
\alpha_{1 \pi}=c_{1 \pi} \frac{f_{\pi N N}^{2}}{f_{A}^{2}}=5.0 \times 10^{-3}
$$

b). The $2 \pi$ mode.

The first process of Eq. 12 is described by diagram (c) of Fig. 17 and is further illustrated in Fig. 19 at the quark level [203]. In Fig. $19 f$ stands for an intermediate fermion, heavy Majorana neutrino, neutralino or gluino.

In the case of $V-A$ theories we find [203]

$$
\alpha_{2 \pi}=\frac{2}{3 g_{A}^{2}} f_{\pi N N}^{2} c_{2 \pi}, \quad c_{2 \pi}=\frac{1}{\sqrt{2 \pi}} \frac{16}{b_{\pi}^{3} m_{p}^{2} m_{\pi}}
$$

The actual value critically depends on the harmonic oscillator size parameter. For $b_{\pi}=0.5 \mathrm{fm}$ we get $\alpha_{2 \pi} \approx 0.1$. 


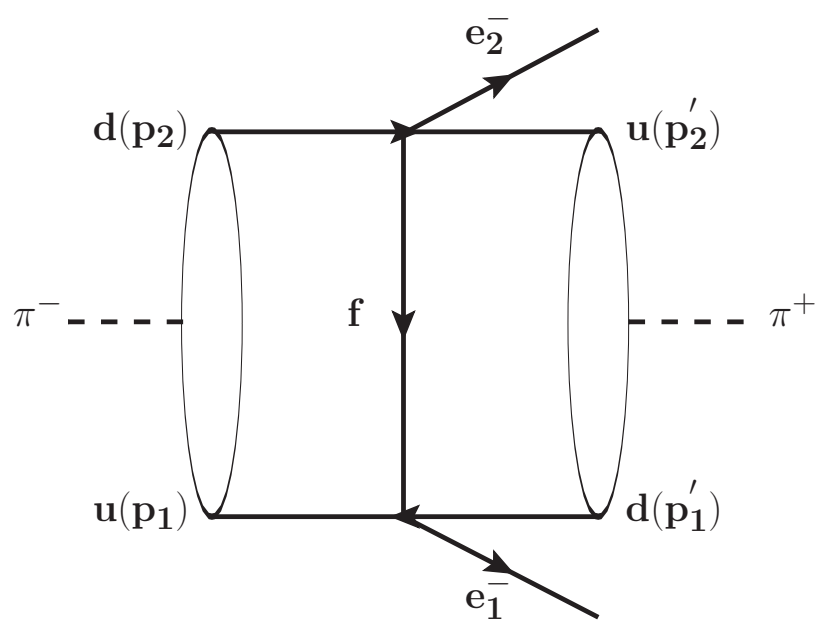

Figure 19: The $0 \nu \beta \beta$-decay of pions in flight ( $2 \pi$ mode of Fig. 17) illustrated at the quark level. $f$ stands for a effective exchange of a heavy Majorana fermion ( heavy neutrino or, as in R-parity violating supersymmetry, a neutralino, gluino etc). The arcs around the pion line merely indicate that the pion is a bound state of two quarks.

In supersymmetry one encounters scalar, pseudoscalar and tensor interactions. For the pseudoscalar interaction the situation is a bit more complicated [203]. One finds

$$
c_{2 \pi}=\frac{1}{\sqrt{2 \pi}} \frac{4}{b_{\pi}^{3} m_{p}^{2} m_{\pi}} \frac{b_{N}^{2}}{b_{\pi}^{2}}\left(\frac{1}{4}\left(\kappa_{d}^{2}+\kappa_{u}^{2}\right)\right), \quad \kappa_{d}=\frac{1}{2 m_{d} b_{N}}, \kappa_{u}=\frac{1}{2 m_{u} b_{N}}
$$

Taking $m_{u}=m_{d}=(1 / 3) m_{p}, b_{N}=1 \mathrm{fm}$ and $b_{\pi}=0.5 \mathrm{fm}$ we find that $\alpha_{2 \pi}$ is much smaller than the value obtained in the elementary particle treatment [93], i.e. we find $\alpha_{2 \pi}=-0.05$. A larger value can be obtained, if one uses the current quark masses. For $m_{u} \approx 2 m_{d}=10 \mathrm{MeV}$ one finds $\alpha_{2 \pi}=-1.3$, but then the validity of non relativistic expansion may be questionable.

\section{Experimental aspects of double beta decays}

\subsection{Progress of DBD experiments}

8.1.1. Experimental aspects of neutrinoless double beta decays Neutrinoless double beta decays are concerned with fundamental properties of neutrinos and weak interactions, which bear some signatures of the high energy scale, and are of great interest from the view point of particle physics and cosmology, as it has been described in previous sections. On the other hand, DBD processes are nuclear rare-decays in the low energy scale, which are studied experimentally by low-energy and low-background nuclear spectroscopy, as given in review articles [31, 62, 63, 30].

Since double beta decays are low-energy second-order weak processes, the decay rates of $2 \nu \beta \beta$ within the SM are of the order of $10^{-20} / \mathrm{y}$, and the rates of $0 \nu \beta \beta$-decay beyond the SM are even much smaller than $2 \nu \beta \beta$-decay rates, depending on the assumed 
light neutrino mass in the case of the Majorana neutrino mediated process. Then the expected $0 \nu \beta \beta$ half-lives are of the order of $T_{1 / 2}^{0 \nu} \approx 10^{27} \mathrm{y}$ and $10^{29} \mathrm{y}$ in the case of the $\mathrm{IH}$ (inverted hierarchy) mass of $30 \mathrm{meV}$ and the $\mathrm{NH}$ (normal hierarchy) mass of $3 \mathrm{meV}$, respectively.

For experimental studies of such rare decays, large detectors with ton-scale DBD isotopes are needed to get $0 \nu \beta \beta$-decay signals in case of the IH neutrino mass. Here, the signals are very rare and occur as low as $E_{\beta \beta} \approx 2-3 \mathrm{MeV}$. Background (BG) signal rates, however, are huge in the energy region of $E_{B} \leq 3 \mathrm{MeV}$. Thus it is crucial to build ultra low BG detectors to find the rare and small $0 \nu \beta \beta$-decay signals among huge BGs in the low energy region.

In spite of this, double beta decays have several unique features that make it realistic to search for the low-energy ultra-rare $0 \nu \beta \beta$ signals among huge BGs.

i. Since $\beta \beta$ half-lives of the order of $T_{1 / 2} \approx 10^{19}-10^{21} \mathrm{y}$ are 10 orders of magnitude longer than the age of the earth, the $\beta \beta$ isotopes are available as almost stable isotopes on the earth, and it is possible to get ton-scale $\left(10^{28}\right) \beta \beta$ isotopes in order to observe some rare $0 \nu \beta \beta$-decay events with $T_{1 / 2}^{0 \nu} \approx 10^{27} \mathrm{y}$.

ii. There are even-even nuclei, where the double beta decays are allowed due to the pairing interaction, but single $\beta$-decays are energetically forbidden. Using such DBD nuclei, one can be free from huge single $\beta$ BGs, which would be larger than $\beta \beta$ rates by factors around $10^{30}$.

iii. The $0 \nu \beta \beta$-decay process with a virtual Majorana neutrino exchange between two nucleons in a nucleus is greatly enhanced because the nucleons are close to each other in the nucleus. Then it is feasible to access the small neutrino masses of the orders of $\delta m_{\mathrm{SUN}}(\operatorname{solar} \nu)-\delta m_{\mathrm{ATM}}(\operatorname{atmospheric} \nu)$.

iv. High energy-resolution and/or correlation studies of $\beta \beta$ rays select low-energy rare $\beta \beta$ signals from huge BG events. There are several DBD nuclei to be studied to confirm ultra-rare $0 \nu \beta \beta$-decay events and possible DBD processes.

Then $\beta \beta$ experiments studies neutrino properties in nuclei, which are around $10^{-15}$ $\mathrm{m}$ in diameter. Thus the $\beta \beta$ nuclei are regarded as excellent femto $\left(10^{-15}\right)$ laboratories with a large filtering power to reject single $\beta$ and other RI BG signals and a large enlargement factor to enhance the $0 \nu \beta \beta$-decay signal of the neutrino physics interest. In the nuclear femto laboratory, two nucleons collide with each other. The luminosity is around $L=10^{48} \mathrm{~cm}^{-2} \mathrm{~s}^{-1}$ in a single DBD isotope. Then the summed luminosity for one ton $\left(10^{28}\right)$ isotopes is around $10^{76} \mathrm{~cm}^{-2} \mathrm{~s}^{-1}$. The cross section for exchange of the Majorana neutrinos with IH mass of $40 \mathrm{meV}$ is of the order of $10^{-83} \mathrm{~cm}^{2}$. Then the event rate is around $2-3$ per year. The huge luminosity of the DBD femto collider enables one to search for the utra-rare $0 \nu \beta \beta$ event and the very small neutrino mass [49].

8.1.2. Progresses of DBD experiments. Progresses of DBD experiments are well described in review papers [31, 62, 63, 30, 205] and refs. therein. Here brief remarks on 
the progresses are given.

Early experimental studies of $\beta \beta$-decays were made by geochemical methods by measuring the number of the $\beta \beta$-decay products. They are inclusive measurements of both the $2 \nu \beta \beta$ and $0 \nu \beta \beta$-decay rates to the ground and excited states. In most realistic cases, the geochemistry methods give the $2 \nu \beta \beta$-decay rate to the ground state because $0 \nu \beta \beta$-decay rates are much reduced due to the small neutrino mass and decays to excited states are disfavored due to the small phase space volume.

Advanced mass spectroscopy was used to measure the number of $\beta \beta$ product isotopes accumulated for the long geological period of around $10^{6-7}$ years in old ores, and to evaluate the long half-lives of the orders of $10^{20} \mathrm{y}$. Extensive studies have been made on such rare-gass isotopes as ${ }^{82} \mathrm{Kr},{ }^{128} \mathrm{Xe}$, and ${ }^{130} \mathrm{Xe}$, which are $\beta \beta$-decay products of ${ }^{82} \mathrm{Se}{ }^{128} \mathrm{Te}$, and ${ }^{130} \mathrm{Te}$, respectively, as described in the review articles. Among them three groups have obtained half lives of around $10^{21}$ for ${ }^{130} \mathrm{Te}$ [13, 11, 12, 14].

Direct counter experiments of $\beta \beta$ decays are exclusive measurements to identify $0 \nu \beta \beta$-decay signals. They have been made by coincidence counter measurements of two $\beta$ rays or by two $\beta$ measurements with tracking chambers, as described in review articles.

High-sensitivity counter experiments were made by using detectors as $\beta \beta$ sources. Der Mateosian and Goldharber have got a limits on the ${ }^{48} \mathrm{Ca} 0 \nu \beta \beta$-decay rate by using large $\mathrm{CaF}_{2}$ scintillators [206]. Stringent limits on the ${ }^{76} \mathrm{Ge} 0 \nu \beta \beta$-decay rate were obtained with high energy-resolution Ge semiconductor detectors by Fiorini et al [207, 208]. Coincidence measurements of two $\beta$ tracks with spark and streamer chambers were made to get limits on the ${ }^{48} \mathrm{Ca}$ and ${ }^{82} \mathrm{Se} 0 \nu \beta \beta$ rays [209, 210, 211, 212].

In 1980's, the Ge experiments for ${ }^{76} \mathrm{Ge} 0 \beta \beta$-decays are improved much by the Milano group [213] and Avignone et al [214]. Ejiri et al used the ELEGANT III with a Ge detector surrounded by Nal scintillators to get limits on the ${ }^{76} \mathrm{Ge} 0 \nu \beta \beta$-decays to the ground and excited states [215, 216, 217].

The first measurement of the $2 \nu \beta \beta$ rays by the direct counting method was made on ${ }^{82}$ Se by Elliott, Hahn and Moe [32]. The observed rate agrees with the $\beta \beta$ rate measured previously by the geochemical method [218, 219]. The first measurement of the $2 \nu \beta \beta$-decay rate by the direct counting method alone on ${ }^{100} \mathrm{Mo}$, where no geochemical measurement was made beforehand, was carried out by the ELEGANT group (Ejiri et al.) [220, 221].

So far, high-sensitivity counter experiments with the mass sensitivities of the orders of $\mathrm{eV}$ and sub $\mathrm{eV}$ have been made on several $\beta \beta$-decay nuclei, and half lives of $2 \nu \beta \beta$ decay rates on many nuclei have been measured by direct counting methods, as reported in the review papers [31, 30, 205]. Recent experiments are discussed in 8.3.

\subsection{Methods and detectors for DBD experiments}

8.2.1. Methods for DBD experiments. Double beta decays proceed normally through the $2 \nu \beta \beta$-decay process within the SM. Transition rates of the $0 \nu \beta \beta$-decay processes 
beyond SM are much rarer than those of the $2 \nu \beta \beta$-decay process in most cases. It is thus necessary to separate experimentally the $0 \nu \beta \beta$-decay processes from the $2 \nu \beta \beta$ decay process.

Geochemical methods counts the number of the decay product isotopes in ores of DBD nuclei for geological time of $T_{1 / 2} \approx 10^{6-7}$ years, which are mostly due to the $2 \nu \beta \beta$-decay process and $0 \nu \beta \beta$-decay processes are not separated from the $2 \nu \beta \beta$-decay process.

Direct counting methods have been extensively used for measuring various DBD processes. The $0 \nu \beta \beta$-decay processes are identified from the sum energy spectrum of $E_{\beta \beta}=E\left(\beta_{1}\right)+E\left(\beta_{2}\right)$ for two $\beta$ rays, as shown in Fig. 2 in Ref. [30]. They show a sharp peak characteristic of the 2-body kinematics at $E_{\beta \beta}=Q_{\beta \beta}$, while $2 \nu \beta \beta$ shows a continuum spectrum characteristic of the 4-body kinematics. Neutrinoless DBD followed by the Majoron (see section 5.3), which is the Goldstone boson associated with spontaneous breaking of $B-L$ symmetry, shows the spectrum characteristic of the 3-body kinematics.

The $0 \nu \beta \beta$-decay processes due to the left handed weak current and the righthanded one (RHC) are experimentally identified by measuring the energy and angular correlations of the two $\beta$ rays, as shown in Fig.4 in ref. 30].

The left handed weak current $0 \nu \beta \beta$ process includes several modes such as the light $\nu$ exchange, the heavy $\nu$ exchange, the SUSY particle exchange, and others, as discussed in the previous sections. Relative contributions of these modes to the $0 \nu \beta \beta$-decay rate may be investigated by observing several DBD isotopes as well as those for the ground and excited states, provided that the matrix elements are properly evaluated. Then experimental studies of several DBD isotopes are necessary.

8.2.2. Sensitivity of $D B D$ experiment $\mathrm{DBD}$ event rates are so low that $\mathrm{DBD}$ experiments are necessarily be carried out by using high-sensitivity detectors at lowbackground underground laboratories.

In case of the $0 \nu \beta \beta$-decay process with the light Majorana neutrino exchange, the transition rate $\Gamma^{0 \nu}$ per year $(\mathrm{y})$ per 1 ton (t) of DBD isotopes is expressed in terms of the nuclear sensitivity $S_{n}$ and the effective mass of the light Majorana neutrinos of $\left|\left\langle m_{\nu}\right\rangle\right|$ as

$$
\Gamma^{0 \nu}=\left|\left\langle m_{\nu}\right\rangle\right|^{2} S_{n}
$$

The nuclear sensitivity is written as

$$
S_{n}=(78 \mathrm{meV})^{-2}\left|\mathrm{M}_{\nu}^{0 \nu}\right|^{2} \mathrm{G}^{0 \nu}(0.01 \mathrm{~A})^{-1}
$$

where $M^{0 \nu}$ is the nuclear matrix element, $A$ is the mass number, $G^{0 \nu}$ is the phase space factor in units of $10^{-14} / \mathrm{y}$.

The mass sensitivity $\left|\left\langle m_{\nu}\right\rangle\right|$ is defined as the minimum mass to be measured by the $0 \nu \beta \beta$-decay experiment. It is expressed in terms of the detector sensitivity $D$ as follows:

$$
\left|\left\langle m_{m}\right\rangle\right|=S_{n}^{-1 / 2} D^{-1 / 2}, \quad D=(\epsilon N T)(\delta)^{-1},
$$


where $\epsilon$ is the $0 \nu \beta \beta$ peak efficiency, $N$ is the number of the DBD isotopes in unit of ton, $T$ is the run time in unit of year and $\delta$ is the minimum counts required for the peak identification with $90 \% \mathrm{CL}$ (confidence level). It is given as $\delta \approx 1.6+1.7(B N T)^{1 / 2}$ with $B$ being the $\mathrm{BG}$ rate $/ \mathrm{t} / \mathrm{y}$, and $\delta \approx 2.3$ for $B N T \geq 2$ and $\leq 2$, respectively.

The nuclear sensitivity $S_{n}$ is proportional to the phase space factor $G^{0 \nu}$ and $\left|M_{\nu}^{0 \nu}\right|^{2}$. Thus DBD nuclei with large $G^{0 \nu}$ and large $M_{\nu}^{0 \nu}$ are selected for the high nuclear sensitivity. DBD detectors with a large efficiency $\epsilon$, a large amount $(N)$ of isotopes, and a small $\mathrm{BG}$ rate $(B)$ are used for the high detector sensitivity.

8.2.3. $D B D$ detectors Neutrinoless $\beta^{-} \beta^{-}$decays of $(A, Z) \rightarrow(A, Z+2)$ are studied by measuring two $\beta^{-}$rays, while neutrinoless $\beta^{+} \beta^{+}$decays of $(A, Z) \rightarrow(A, Z-2)$ proceed through $\beta^{+} \beta^{+}, \beta^{+} \mathrm{EC}$, and $\mathrm{EC} \mathrm{EC} \gamma$, where $\mathrm{EC}$ (electron capture) is detected by measuring the $\mathrm{X}$ ray, and $\beta^{+}$by measuring the $\beta^{+}$and the two $511 \mathrm{keV}$ annihilation $\gamma$ rays.

High-sensitivity DBD experiments require DBD isotopes with high nuclear sensitivity $S_{n}$, i.e. the large $Q_{\beta \beta}$ and the large phase space factor $G^{0 \nu}$, as given by Eq. (129).

There are several such DBD isotopes of $\beta^{-} \beta^{-}$decays. However, no $\beta^{+} \beta^{+}$nuclei with large $S_{n}$ are available, although BG rates for the $\beta^{+} \beta^{+}$and $\beta^{+}$EC are quite small by measuring both $\beta^{+}$and the annihilation $\gamma$ rays and/or the $\mathrm{X}$ ray. Accordingly most of high-sensitivity DBD experiments are concentrated on the $0 \nu \beta^{-} \beta^{-}$decays with the large $Q_{\beta \beta}$ and $G^{0 \nu}$. Thus hereafter, we discuss mainly $\beta^{-} \beta^{-}$decays. The large $Q_{\beta \beta} \approx 3$ $\mathrm{MeV}$ helps reduce BG rates since most of BGs from natural RIs are below $3 \mathrm{MeV}$.

The $0 \nu \beta^{-} \beta^{-}$experiments with the IH $(30 \mathrm{meV})$ mass sensitivity are carried out by using low $\mathrm{BG}(B \approx 1 / \mathrm{t}$ y) detectors and ton-scale $(N \approx 0.5-1)$ DBD isotopes with the large nuclear matrix element of $\left|M_{\nu}^{0 \nu}\right| \approx 3$ (see Table 3) and the large phase space factor of $G^{0 \nu} \approx 5$ in unit of $10^{-14} / \mathrm{y}$ for a long $(T \approx 2-4$ year) run time. On the other hand one needs DBD isotopes of around $N \approx 50-100$ ton and ultra-low BG detectors with $B \approx 0.01 / \mathrm{t} / \mathrm{y}$ to reach the $\mathrm{NH}(2-4 \mathrm{meV})$ mass sensitivity.

Possible DBD isotopes to be used for high-sensitivity $0 \nu \beta^{-} \beta^{-}$experiments are given in Table 1. Among them, ${ }^{82} \mathrm{Se},{ }^{100} \mathrm{Mo},{ }^{116} \mathrm{Cd},{ }^{130} \mathrm{Te},{ }^{136} \mathrm{Xe}$ have the large $Q_{\beta \beta} \approx 3 \mathrm{MeV}$ and the large $G^{0 \nu} \approx 5$ in unit of $10^{-14} / \mathrm{y}$, as shown in Table 1, ${ }^{130} \mathrm{Te}$ has the largest abundance ratio of $34.5 \%$, while others have the abundance ratios of around $10 \%$, and are enriched to $85-90 \%$ by means of centrifugal separation.

${ }^{76} \mathrm{Ge}$ is a special case with smaller $Q_{\beta \beta} \approx 2 \mathrm{MeV}$ and the smaller $G^{0 \nu} \approx 0.71$, but high sensitivity experiments are possible by using low-BG ${ }^{76} \mathrm{Ge}$ detectors with high energy-resolution [207, 208]. ${ }^{150} \mathrm{Nd}$ has a very large phase space factor of $G^{0 \nu} \approx 23.2$ in units of $10^{-14} / \mathrm{y}$, but the natural abundance ratio is only $5.6 \%$, and the enrichment is hard. The matrix element may be reduced because of the large difference in nuclear shape between the initial and final nuclei.

DBD experiments are carried out by using either calorimetric detectors or spectroscopic detectors. Calorimetric detectors are made partly of DBD isotopes, and 
thus the detection efficiency is as large as $\epsilon \approx 0.6-0.9$. Cryogenic detectors with Te [222], semiconductor detectors with ${ }^{76} \mathrm{Ge}$ and ${ }^{116} \mathrm{Cd}$, and scintillation detectors with ${ }^{116} \mathrm{Cd}$ and ${ }^{136} \mathrm{Xe}$ are used as calorimetric detectors.

Cryogenic bolometers of $\mathrm{ZnS}, \mathrm{CaMoO}_{4}, \mathrm{ZnMoO}_{4}$ and others are shown to be used as high energy-resolution DBD detectors. BG rates of these detectors are reduced by measuring both thermal signals as well as scintillation signals [223, 224, 225].

Spectroscopic tracking detectors with DBD isotopes outside the detectors are used to measure individual $\beta$ rays from the isotopes. Then the low BG measurements are possible even though the efficiency is low and the energy resolution is modest. ${ }^{82} \mathrm{Se}$ and ${ }^{100} \mathrm{Mo}$ are studied by spectroscopic detectors with PL (plastic scintillator) arrays. Spectroscopic detectors measure $\beta-\beta$ energy and angular correlations, which are used to confirm and identify the $0 \nu \beta \beta$-decay process.

So far, high-sensitivity DBD experiments have been made mainly on the ground state $0^{+}$transition because of the large phase space factor. Transitions to the excited $0^{+}$states are experimentally measured in coincidence with $\gamma$ rays. Then BG rates are much reduced, even though the phase space factors are smaller by almost one order of magnitude. Measurements of both the ground and excited transitions are interesting to confirm the $0 \nu \beta \beta$-decay signal and to study the $0 \nu \beta \beta$ mechanism. The excited $0^{+}$ states in ${ }^{82} \mathrm{Se},{ }^{100} \mathrm{Mo},{ }^{136} \mathrm{Xe}$, and ${ }^{150} \mathrm{Nd}$ are located in low excitation region. Some excited states are different in shape from the ground state. Thus $0 \nu \beta \beta$-decay measurements for both the ground and excited states are very interesting.

\subsection{Present status and future projects of DBD experiments}

8.3.1. Neutrinoless double beta decays Experimental studies of $0 \nu \beta \beta$-decay have been carried out on several $\beta \beta$ nuclei [30, 31, 49]. Some of them by counter measurments are listed in Table 1, ${ }^{128} \mathrm{Te}$ was studied by a geochemical method, and the hallife and mass limits are $7.7 \pm 10^{24} \mathrm{y}$ and $1.1-1.5 \mathrm{eV}$ [226]. Here the effective mass given in the 6th column shows a range of the values evaluated from the experimental half-life by using various matrix elements. In fact the mass depends much on the matrix element as discussed in section 10. Calorimetric detectors have been used for isotopes such as ${ }^{48} \mathrm{Ca}$, ${ }^{76} \mathrm{Ge},{ }^{116} \mathrm{Cd}$ and ${ }^{130} \mathrm{Te}$. Among them, ${ }^{76} \mathrm{Ge}$ experiments (Heidelberg-Moscow, IGEX) with Ge semiconductors [36, 227, 228, 229, 230] and the ${ }^{130}$ Te experiment(CUORICINO) with $41 \mathrm{~kg} \mathrm{TeO}{ }_{2}$ cryogenic bolometers [231, 232] have good energy-resolution and give stringent limits on the absolute value of effective Majorana neutrino mass of the order of $0.3-0.5 \mathrm{eV}$. Currently, the most stringent limit on $\left|\left\langle m_{\nu}\right\rangle\right|$ comes from the lower limit on the $T_{1 / 2}^{0 \nu}\left({ }^{136} \mathrm{Xe}\right)$ measured in KamLAND-Zen experiment [40] (see Table 1).

Spectroscopic detectors (ELEGANT V, NEMO III) have been used for ${ }^{82} \mathrm{Se},{ }^{100} \mathrm{Mo}$, ${ }^{116} \mathrm{Cd}$ and other isotopes with large $Q_{\beta \beta}$ values [38, 233, 234, 235]. They are $\beta$-ray tracking detectors with $\beta \beta$ sources separated from detectors. NEMO III provides stringent limits on the $0 \nu \beta \beta$ half-lives for ${ }^{82} \mathrm{Se},{ }^{100} \mathrm{Mo}$, and other isotopes [38, 234, 235.

Recently, a claim for the $0 \nu \beta \beta$-decay peak, corresponding to the effective Majorana 
Table 1: Limits on neutrinoless double beta decays $T_{1 / 2}^{0 \nu-e x p}$ (claim for evidence is denoted with upper index c). $Q_{\beta \beta}: Q$ value for the $0^{+} \rightarrow 0^{+}$ground state transition. $G^{0 \nu}$ : kinematical (phase space volume) factor $\left(g_{A}=1.25\right.$ and $\left.\mathrm{R}=1.2 \mathrm{fm} A^{1 / 3}\right) .\left\langle m_{\nu}\right\rangle$ : The upper limit on the effective Majorana neutrino mass, deduced from $T_{1 / 2}^{0 \nu-e x p}$ by assuming the ISM [236] $\left(g_{A}^{\text {eff }}=1.25\right.$, UCOM src $)$, the EDF [131] $\left(g_{A}^{\text {eff }}=1.25\right.$, UCOM src $)$, the (R)QRPA $\left(1.00 \leq g_{A}^{e f f} \leq 1.25\right.$, the modern self-consistent treatment of src), and the IBM-2 [130] $\left(1.00 \leq g_{A}^{e f f} \leq 1.25\right.$, Miller-Spencer src), nuclear matrix elements (see section 10). src means short-range correlations.

\begin{tabular}{|c|c|c|c|c|c|c|c|}
\hline isotope & $\begin{array}{c}A \\
{[\%]}\end{array}$ & $\begin{array}{c}Q_{\beta \beta} \\
{[\mathrm{MeV}]}\end{array}$ & $\begin{array}{c}G^{0 \nu} \\
{\left[10^{-14} \mathrm{y}\right]}\end{array}$ & $\begin{array}{l}T_{1 / 2}^{0 \nu-\exp } \\
{\left[10^{24} \mathrm{y}\right]}\end{array}$ & $\mathrm{NME}$ & $\begin{array}{c}\left\langle m_{\nu}\right\rangle \mid \mathrm{eV} \\
{[\mathrm{eV}]}\end{array}$ & $\begin{array}{c}\text { Future } \\
\text { experiments }\end{array}$ \\
\hline \multirow[t]{2}{*}{${ }^{48} \mathrm{Ca}$} & 0.19 & 4.276 & 7.15 & $0.014^{a}$ & ISM & 19.1 & CANDLES \\
\hline & & & & & $\mathrm{EDF}$ & 7.0 & \\
\hline \multirow[t]{9}{*}{${ }^{76} \mathrm{Ge}$} & 7.8 & 2.039 & 0.71 & $19^{b}$ & ISM, EDF & $0.51,0.31$ & GERDA \\
\hline & & & & & (R)QRPA & $(0.20,0.32)$ & \\
\hline & & & & & $\mathrm{EDF}$ & $(0.26,0.35)$ & \\
\hline & 7.8 & 2.039 & 0.71 & $22^{c}$ & ISM, EDF & $0.47,0.29$ & - \\
\hline & & & & & (R)QRPA & $(0.18,0.30)$ & \\
\hline & & & & & $\mathrm{EDF}$ & $(0.24,0.32)$ & \\
\hline & 7.8 & 2.039 & 0.71 & $16^{d}$ & ISM, EDF & $0.55,0.34$ & MAJORANA \\
\hline & & & & & (R)QRPA & $(0.22,0.35)$ & \\
\hline & & & & & $\mathrm{EDF}$ & $(0.28,0.38)$ & \\
\hline \multirow[t]{3}{*}{${ }^{82} \mathrm{Se}$} & 9.2 & 2.992 & 3.11 & $0.36^{e}$ & ISM, EDF & $1.88,1.17$ & SuperNEMO \\
\hline & & & & & (R)QRPA & $(0.76,1.28)$ & $\mathrm{MOON}$ \\
\hline & & & & & $\mathrm{EDF}$ & $(1.12,1.49)$ & \\
\hline \multirow[t]{3}{*}{${ }^{100} \mathrm{Mo}$} & 9.6 & 3.034 & 5.03 & $1.0^{f}$ & $\mathrm{EDF}$ & 0.46 & MOON \\
\hline & & & & & (R)QRPA & $(0.38,0.73)$ & AMoRE \\
\hline & & & & & $\mathrm{EDF}$ & $(0.62,1.06)$ & \\
\hline \multirow[t]{2}{*}{${ }^{116} \mathrm{Cd}$} & 7.5 & 2.804 & 5.44 & $0.17^{g}$ & $\mathrm{EDF}$ & 1.15 & COBRA \\
\hline & & & & & (R)QRPA & $(1.20,2.16)$ & $\mathrm{CdWO}_{4}$ \\
\hline \multirow[t]{3}{*}{${ }^{130} \mathrm{Te}$} & 34.5 & 2.529 & 4.89 & $3.0^{h}$ & ISM, EDF & $0.52,0.27$ & CUORE \\
\hline & & & & & (R)QRPA & $(0.25,0.43)$ & \\
\hline & & & & & EDF & $(0.33,0.46)$ & \\
\hline \multirow[t]{2}{*}{${ }^{136} \mathrm{Xe}$} & 8.9 & 2.467 & 5.13 & $5.7^{i}$ & ISM, EDF & $0.44,0.23$ & EXO, NEXT \\
\hline & & & & & (R)QRPA & $(0.17,0.30)$ & KamLAND-Zen \\
\hline \multirow[t]{2}{*}{${ }^{150} \mathrm{Nd}$} & 5.6 & 3.368 & 23.2 & $0.018^{j}$ & EDF & 4.68 & SuperNEMO \\
\hline & & & & & (R)QRPA & $(2.13,2.88)$ & $\mathrm{SNO}+\mathrm{DCBA}$ \\
\hline
\end{tabular}

a:[237], b:[36, 227, 228], c:[42], d:[229, 230], e:[38, 234, 235], f:[38, 234], g:[238], h:[231, 232, 239], i: 40], j: [38, 240]. 
neutrino mass of $0.32 \mathrm{eV}$, was made by a part of the Heidelberg-Moscow collaboration [41, 42]. The result depends on the off-line analysis method. It should be checked by future GERDA/MAJORANA experiments with lower BG rates.

Neutrino-mass sensitivities of the CUORICINO and NEMO III detectors are limited to be around $300-500 \mathrm{meV}$ because of the limited $\beta \beta$ isotopes of $11-7 \mathrm{~kg}$ and the large BG rates. Thus future experiments with higher mass sensitivity are necessary to prove/disprove the Heidelberg-Moscow claim and to search for the Majorana neutrino in the lower $\nu$ mass regions.

The neutrino oscillation studies have given a strong impact to high-sensitivity studies of $\beta \beta$ experiments since the effective mass suggested is of the order of $\sqrt{ } \delta m^{2} \sim$ $2 \mathrm{meV}-50 \mathrm{meV}$, which next-generation $\beta \beta$ detectors can access if the $\nu$ 's are Majorana particles. Future experiments with higher mass sensitivities are in progress (see below).

8.3.2. Two neutrino double beta decays The $2 \nu \beta \beta$-decay is a process fully consistent with the SM of electroweak interaction. The inverse half-life of $2 \nu \beta \beta$-decay is free of unknown parameters on the particle physics. It can be expressed as a product of an accurately known phase-space factor $G^{2 \nu}\left(E_{0}, Z\right)$, which includes fourth power of $g_{A}$, and the double Gamow Teller transition matrix element $M^{2 \nu}(A, Z)$, which is a quantity of the second order in the perturbation theory:

$$
\left(T_{1 / 2}^{2 \nu}\right)^{-1}=G^{2 \nu}\left(E_{0}, Z\right)\left|M^{2 \nu}(A, Z)\right|^{2} .
$$

Here $M^{2 \nu}$ includes nuclear effects due to the nuclear residual interactions, the nuclear medium and the nuclear quenching. It is obtained from the experimental decay rate. Experimental studies of $2 \nu \beta \beta$-decay half-lives have been made on some nuclei by geochemical methods, and several nuclei by direct counting methods [241]. The $2 \nu \beta \beta$ decay has been observed so far in 12 nuclides $\left({ }^{48} \mathrm{Ca},{ }^{76} \mathrm{Ge}{ }^{82} \mathrm{Se},{ }^{96} \mathrm{Zr},{ }^{100} \mathrm{Mo},{ }^{116} \mathrm{Cd}\right.$, ${ }^{128} \mathrm{Te},{ }^{130} \mathrm{Te},{ }^{136} \mathrm{Xe},{ }^{150} \mathrm{Nd},{ }^{130} \mathrm{Ba}$ and ${ }^{238} \mathrm{U}$ ) and in two excited states [241]. Recent NEMO III experiments provide high-statistic spectroscopic studies of the $2 \nu \beta \beta$-decay rates [38, 240]. Spectroscopic measurements of two $\beta$ rays are useful to reduce BG rates and energy correlations of two $\beta$ rays are used to identify the $2 \nu \beta \beta$ mechanism.

The measurement of $2 \nu \beta \beta$-decay rates gives us information about the product of the squared effective axial-vector coupling constant and $2 \nu \beta \beta$-decay matrix elements. They are presented in Table 2, The $2 \nu \beta \beta$-decay matrix elements are sensitive to nuclear-spin isospin correlations. The observed values for $M^{2 \nu}$ are used to investigate the nuclear structure and the nuclear interactions associated with the $0 \nu \beta \beta$-decays.

8.3.3. High sensitivity experiments In the case of the inverted mass hierarchy, $\beta \beta$ detectors with the IH mass sensitivity of $\left\langle m_{m}\right\rangle \approx 20-50 \mathrm{meV}$ can be used to study the $0 \nu \beta \beta$-decay, while in the case of the normal hierarchy one needs higher sensitivity detectors with $\left\langle m_{m}\right\rangle \approx 2-4 \mathrm{meV}$. Several groups are now working for next-generation $\beta \beta$ experiments with the IH mass sensitivities of $20 \sim 50 \mathrm{meV}$, as discussed in the reviews [30, 31, 183]. 
Table 2: The $2 \nu \beta \beta$ matrix elements $\left|M^{2 \nu}\right|$ deduced from the measured half-life $T_{1 / 2}^{2 \nu}$ by counter experiments [241] [40]. $g_{A}=1.269$ is assumed.

\begin{tabular}{lcc}
\hline \hline Nucleus & $T_{1 / 2}^{2 \nu}$ years & $\left|M^{2 \nu}\right|(\mathrm{MeV})^{-1}$ \\
\hline${ }^{48} \mathrm{Ca}$ & $4.4_{-0.5}^{+0.6} \times 10^{19}$ & $0.046_{-0.003}^{+0.003}$ \\
${ }^{76} \mathrm{Ge}$ & $1.5_{-0.1}^{+0.1} \times 10^{21}$ & $0.137_{-0.004}^{+0.005}$ \\
${ }^{82} \mathrm{Se}$ & $9.2_{-0.7}^{+0.7} \times 10^{19}$ & $0.095_{-0.003}^{+0.004}$ \\
${ }^{96} \mathrm{Zr}$ & $2.3_{-0.2}^{+0.2} \times 10^{19}$ & $0.091_{-0.004}^{+0.004}$ \\
${ }^{100} \mathrm{Mo}$ & $7.1_{-0.4}^{+0.4} \times 10^{18}$ & $0.234_{-0.006}^{+0.007}$ \\
${ }^{100} \mathrm{Mo}{ }^{*}$ & $5.9_{-0.6}^{+0.8} \times 10^{20}$ & $0.189_{-0.012}^{+0.010}$ \\
${ }^{116} \mathrm{Cd}$ & $2.8_{-0.2}^{+0.2} \times 10^{19}$ & $0.128_{-0.004}^{+0.005}$ \\
${ }^{128} \mathrm{Te}$ & $1.9_{-0.4}^{+0.4} \times 10^{24}$ & $0.047_{-0.003}^{+0.007}$ \\
${ }^{130} \mathrm{Te}$ & $6.8_{-1.1}^{+1.2} \times 10^{20}$ & $0.034_{-0.003}^{+0.003}$ \\
${ }^{136} \mathrm{Xe}$ & $2.38_{-0.14}^{+0.14} \times 10^{21}$ & $0.018_{-0.001}^{+0.003}$ \\
${ }^{150} \mathrm{Nd}$ & $8.2_{-0.9}^{+0.9} \times 10^{18}$ & $0.061_{-0.003}^{+0.004}$ \\
${ }^{150} \mathrm{Nd}{ }^{*}$ & $1.33_{-0.26}^{+0.45} \times 10^{20}$ & $0.045_{-0.006}^{+0.005}$ \\
\hline \hline
\end{tabular}

Experimental proposals for future $\beta \beta$ experiments have been made on several $\beta \beta$ isotopes, and some of them are listed in Table 1. They are mostly $\beta^{-} \beta^{-}$experiments because of large kinematical (phase space) factors. DBD experiments with different isotopes and different methods are indispensable to confirm and identify the $0 \nu \beta \beta$-decay event and the $0 \nu \beta \beta$-decay mechanism. Some of them are briefly described below.

${ }^{76} \mathrm{Ge}$ experiments with low-BG high resolution ${ }^{76} \mathrm{Ge}$ detectors are of special interest for proving or disproving the Heidelberg-Moscow claim of the large $0 \nu \beta \beta$-decay peak [42], and for further high-sensitivity ton-scale experiments.

GERDA: It aims at high energy-resolution studies of ${ }^{76} \mathrm{Ge} 0 \nu \beta \beta$-decays by using high-purity ${ }^{76} \mathrm{Ge}$ detectors to check the Heidelberg-Moscow claim and the possible Majorana neutrinos in the QD region at LNGS (Gran Sasso). GERDA uses $18 \mathrm{~kg}$ ${ }^{76} \mathrm{Ge}$ detectors in Phase I, and add $20 \mathrm{~kg}$ detectors in Phase II. The Ge detectors are immersed into high purity liquid Ar in order to avoid BG contributions from cryostats [175]. GERDA is now running.

MAJORANA: The MAJORANA demonstrator uses $40 \mathrm{~kg}$ Ge detectors at the Sanford underground lab. to test/investigate the half life of the Heidelberg-Moscow claim and the QD mass regions and to prove the feasibility for a future ton-scale IH $\left(10^{27} \mathrm{y}, 20-40 \mathrm{meV}\right)$ experiment. The Ge detectors are PPC (P-type Point Contact) detectors with excellent PSA(Pulse Shape Analysis). They are cooled by using ultrapure electro-formed $\mathrm{Cu}$ cryostat [179]. The BG goal is $4 / \mathrm{t} \mathrm{y}$, which scales to $1 / \mathrm{t}$ y for the 1-ton experiment. The enriched detectors will be on-line in $2013-2014$. 
These detectors can also be used to study DM and neutrino scattering in the low energy region. The GERDA and the Majorana collaboration will be merged for one ton-scale future experiment by selecting the best techniques developed and tested by GERDA and MAJORANA. Recent developments are given in the report [242].

There are other experimental plans for QD masses. Among them, CANDLES is for ${ }^{48} \mathrm{Ca} \beta \beta$-decays with an array of $\mathrm{CaF}_{2}$ crystals [243], which is based on the ELEGANT VI experiment with $\mathrm{CaF}_{2}$. The $Q_{\beta \beta}$ is large, but the natural abundance of ${ }^{48} \mathrm{Ca}$ is only $0.2 \%$. Thus the efficient isotope enrichment is crucial.

Several groups are working for future experiments with the IH mass sensitivities of around $\left\langle m_{\nu}\right\rangle \approx 20-50 \mathrm{meV}$. DBD isotopes required are those with the large $Q_{\beta \beta}=$ $2.5-3 \mathrm{MeV}$ and $G^{0 \nu}=3-510^{-14} / \mathrm{y}$ to get large nuclear sensitivities of the order of $\left(S_{N}\right)^{-1 / 2}=15-20 \mathrm{meV}$. The experiments use large-scale low-BG detectors with ton-scale enriched isotopes and $B \approx 1 / \mathrm{t} \mathrm{y}$.

MOON (Molybdenum Observatory Of Neutrinos): This is an extension of ELEGANT V [233]. It is a hybrid $\beta \beta$ and solar $\nu$ experiment with ${ }^{100} \mathrm{Mo}$ to study the Majorana $\nu$ masses with the QD - IH mass sensitivities of $100-20 \mathrm{meV}$ and the low energy solar $\nu \mathrm{s}[244,245,246,247,248$. Double beta decays to both the ground and the $1.132 \mathrm{MeV}$ excited $0^{+}$states are studied to confirm the $0 \nu \beta \beta$ events and to study the $0 \nu \beta \beta$-decay mechanism. MOON can be used for supernova neutrinos as well [249].

Detectors under considerations are : A) the super-module of PL plate and fiber scintillators [245, 246, 247] and B) the cryogenic bolometer of $\mathrm{ZnMoO}_{4}$. The PL scintillator module (A) is used for spectroscopic study of two $\beta$-ray energy and angular correlations to identify the $0 \nu \beta \beta$-decay process. Here one module consists of a plate (PL) scintillator for the $\beta$ energy and two sets of X-Y fiber scintillator planes for the vertex identification, between which a thin ${ }^{100} \mathrm{Mo}$ film is interleaved. The energy resolution is $\sigma \approx 2.2 \%$ at $E=Q_{\beta \beta}$ to reduce the $2 \nu \beta \beta$-decay tail in the $0 \nu \beta \beta$-decay window. The half life (mass) sensitivity is $3 \times 10^{26} \mathrm{y}(45 \mathrm{meV})$ with $480 \mathrm{~kg}{ }^{100} \mathrm{Mo}$ for 5 years. Proto-type detectors were built to show the energy resolution as required [245, 246].

The $\mathrm{ZnMoO}_{4}$ bolometer(B), which is under discussion with the Milano-Rome group is for calorimetric study of the sum of two $\beta$-ray energy. The high energy-resolution ( $\Delta E \approx 5 \mathrm{keV}$ ), the particle identification by the scintillation and/or the pulse-shape analysis and the high efficiency $(\epsilon \approx 0.8)$ make the high-sensitivity study possible. Thus it is good to start with the detector $\mathrm{B}$, and proceed to $\mathrm{A}$ to confirm the $0 \nu \beta \beta$-decay process by two $\beta$-ray measurement. The half-life (mass) sensitivities are $4 \times 10^{25}$ y $(120$ $\mathrm{meV})$ for 3 y run with $12 \mathrm{~kg}{ }^{100} \mathrm{Mo}$ and $7 \times 10^{26} \mathrm{y}(30 \mathrm{meV})$ for $5 \mathrm{y}$ run with $220 \mathrm{~kg}$ ${ }^{100} \mathrm{Mo}$.

SuperNEMO: The goal of SuperNEMO is to reach a sensitivity of $10^{26} \mathrm{y}$, which corresponds to the IH mass of 40-110 meV [250]. The detector consists of huge tracking chambers and scintillation detectors with $100 \mathrm{~kg}$ of $\beta \beta$ isotopes of ${ }^{150} \mathrm{Nd}$ or ${ }^{82} \mathrm{Se}$ to search for the $\nu$ mass below $0.1 \mathrm{eV}$. The efficiency is $30 \%$ and the resolution is $4 \%$ in FWHM. The BG impurities are deduced to be ${ }^{208} \mathrm{Tl} \leq 2$ and ${ }^{214} \mathrm{Bi} \leq 10$ in unit of $\mu$ $\mathrm{Bq} / \mathrm{kg}$ It plans to use 20 modules, each module with $5 \mathrm{~kg} \beta \beta$ isotopes. The first module 
is a demonstrator with $7 \mathrm{~kg}$ of ${ }^{82} \mathrm{Se}$. The mass sensitivity of the demonstrator with 15 $\mathrm{kg} \mathrm{y}$ is $210-570 \mathrm{meV}$, while that of the full detector array with $500 \mathrm{~kg}$ y is $53-145 \mathrm{meV}$, depending on the nuclear matrix element. It is based on NEMOIII, and thus it is crucial to improve the energy resolution and the efficiency.

AMoRE Advanced Molybdenum based Rare process Experiment: Large volume $\mathrm{CaMoO}_{4}$ crystals with enriched material have been developed to study the $0 \nu \beta \beta$-decays of ${ }^{100} \mathrm{Mo}$ and to search for cold dark matter [251]. Pilot experiments of $1 \mathrm{~kg}$ with scintillation technique and $\mathrm{Cs}(\mathrm{I})$ active veto are in preparation. In order to improve the energy resolution, cryogenic $\mathrm{CaMoO}_{4}$ detectors are being developed. To avoid BGs from the $2 \nu \beta \beta$-decays of ${ }^{48} \mathrm{Ca}$, depletion of $\mathrm{Ca}$ in ${ }^{48} \mathrm{Ca} \leq 0.001 \%$ is made by using ALSIS (Advanced Laser Stable Isotope Separation). Additional light sensor and time constant of phonon signal are effective to select signals. The goal of AMoRE is to study $0 \nu \beta \beta$ decays of ${ }^{100} \mathrm{Mo}$ in the region of IH mass of $50 \mathrm{meV}\left(310^{26} \mathrm{y}\right)$ by using $100 \mathrm{~kg} \mathrm{CaMoO}_{4}$ cryogenic detectors.

$C O B R A$ : It uses a large amount of high energy-resolution CdZnTe semiconductors at room temperature 252]. The modular design makes coincidence measurements possible to reduce BG rates. The crystal includes several $\beta \beta$ isotopes to be studied. The collaboration now tests $64 \mathrm{CZT} 1 \mathrm{~cm}^{3}$ detectors at LNGS. The goal is to study the Majorana neutrino in the $\mathrm{IH}$ mass region by measuring the $0 \nu \beta \beta$ from ${ }^{116} \mathrm{Cd}$ with $Q_{\beta \beta}$ $=2.809 \mathrm{MeV}$. The detector is composed by $64 \mathrm{~K}$ crystals with 0.42 ton $\mathrm{Cd}$ isotopes enriched in ${ }^{116} \mathrm{Cd}$. Reduction of BGs from RI impurities inside and around detectors are important. Pixelisation(Semiconductor tracker) can be a major step forward.

CUORE (Cryogenic Underground Observatory for Rare Event): This is an expansion of CUORICINO. It is a high energy resolution bolometer array to measure the ${ }^{130} \mathrm{Te} 0 \nu \beta \beta$ decays with $Q=2.529 \mathrm{MeV}$ at LNGS [239]. It uses natural $\mathrm{TeO}_{2}$ crystals with the natural ${ }^{130} \mathrm{Te}$ abundance of $34 \%$. It consists of $988 \mathrm{TeO}_{2}$ crystals with the net ${ }^{130} \mathrm{Te}$ mass of $203 \mathrm{~kg}$. The detector array is under construction since 2005.

The experiment emphases 20 times more massive than CUORETINO, better energy resolution of $5 \mathrm{keV}$, high granularity, and thus low BG rates. The CUORICINO BG rate, which is around $0.16 / \mathrm{keV} / \mathrm{kg} / \mathrm{y}\left(410^{3} / 5 \mathrm{keV} / \mathrm{y} /\right.$ ton of $\left.{ }^{130} \mathrm{Te}\right)$, is expected to be reduced to $0.02-0.01 / \mathrm{keV} / \mathrm{kg} / \mathrm{y}$ in CUORE. Then, in cases of the BG rates of $B=0.01$ - $0.001 / \mathrm{keV} / \mathrm{kg} / \mathrm{y}\left(2.510^{2}-2.510^{1} / 5 \mathrm{keV} / \mathrm{y} /\right.$ ton of $\left.{ }^{130} \mathrm{Te}\right)$, the half-life and the $\nu$ mass sensitivities are expected to be around $2.1-6.510^{26}$ y and $50-25 \mathrm{meV}$, which depend on the nuclear matrix element. The first CUORE tower is CUORE-0.

EXO (Enriched Xenon Observatory): The $\beta \beta$ experiment of ${ }^{136} \mathrm{Xe}$ with $Q=$ $2.467 \mathrm{MeV}$ [253] is made by using the laser tagging technique to select the residual nuclei of ${ }^{136} \mathrm{Ba}$ to suppresses all kinds of RI BGs. The energy-resolution of around $\sigma \sim 2 \%$ is achieved by measuring both the ionization and scintillation signals. The 1 ton enriched Xe detector with the energy-resolution of $\sigma=1.6 \%$ gives the $\nu$-mass sensitivity of $50-70 \mathrm{meV}$ for a $5 \mathrm{y}$ run. The 10 ton Xe detector with the improved energy resolution of $\sigma=1 \%$ will give the sensitivity $\|$ of $11-15 \mathrm{meV}$. The $200 \mathrm{~kg}{ }^{136} \mathrm{Xe}$ liquid \|I In a recent paper the EXO collaboration 254 reported that no signal has appeared in a search for 
detector is used at WIPP to study the $2 \nu \beta \beta$-decay and the quasi-degenerate $\nu$-mass, as the first step without the Ba tagging. EXO observed the $2 \nu \beta \beta$ half life of $2.1 \times 10^{21}$ [255. The key point of this experiment is the tagging efficiency of the ${ }^{136} \mathrm{Ba}$ nuclei.

KamLAND-Zen (Kamioka Large Anti Neutrino Detector Zenon): It studies the ${ }^{136}$ Xe DBD by means of the KamLAND detector with the 1 kton liquid scintillator at Kamioka [256]. A mini balloon with $3.2 \mathrm{~m}$ in diameter is set at the center for the ${ }^{136} \mathrm{Xe}$-loaded liquid scintillator. It includes ${ }^{136} \mathrm{Xe}$ isotopes around $400 \mathrm{~kg}$. The energyresolution and the vertex-resolution are $6.8 \% / \sqrt{ } E$ and $12.5 \mathrm{~cm} / \sqrt{ } E$. The collaboration neasured the $2 \nu \beta \beta$ half-life [40], and hopes to reach the sensitivity of around $50 \mathrm{meV}$.

NEXT (Neutrino Experiment with a Xe TPC): A Xe TPC with 100-150 kg enriched ${ }^{136} \mathrm{Xe}$ is used at LSC [257]. It is a low BG and good E-resolution TPC with separate readout planes for tracking and energy. The NEXT-100 sensitivity for 5 y run is about $5.910^{25}$ y (better than $100 \mathrm{meV}$ ).

Borexino with 2 ton ${ }^{136} \mathrm{Xe}$ : The sensitivity is around $100 \mathrm{meV}$ [258].

DCBA (Drift Chamber Beta-ray Observatory): It uses a tracking chamber in a magnetic field to study ${ }^{150} \mathrm{Nd} \beta \beta$ decays [259]. The $\beta$ energy is obtained by the $\beta$-ray trajectories. DCBA-T3 is now under construction. The good energy-resolution and efficient enrichment of ${ }^{150} \mathrm{Nd}$ isotopes are necessary.

$\mathrm{SNO}+($ Sudbury Neutrino Observatory + ): It uses the $1 \mathrm{k}$ ton scintillation detector with $0.1 \% \mathrm{Nd}$ isotopes to study QD-IH $\nu$ masses by using natural (5.6\%) and enriched $(50 \%){ }^{150} \mathrm{Nd}$ isotopes [260]. The mass sensitivities are $100 \mathrm{meV}$ with the natural (5.6 $\%{ }^{150} \mathrm{Nd} 56 \mathrm{~kg}$ ) and $40 \mathrm{meV}$ with enriched isotopes (50\% enriched ${ }^{150} \mathrm{Nd} 500 \mathrm{~kg}$ ). The collaboration is trying to find a realistic way of the $\mathrm{Nd}$ isotope separation, which is of great interest to study the IH neutrino mass. It aims at the scintillator filling at the beginning of 2013.

DBD experiments for NH mass: Higher sensitivity DBD experiments for NH masses of $\left\langle m_{\nu}\right\rangle=2-4 \mathrm{meV}$ require a large amount of high nuclear-sensitivity $\left(S_{n} \approx 15 \mathrm{meV}\right)$ DBD isotopes of the order of $N \approx 10-50$ tons, and extremely low BG detectors with $B \leq 0.1-0.01 / \mathrm{t}$ y. DBD isotopes to be studied are ${ }^{82} \mathrm{Se},{ }^{100} \mathrm{Mo}$, and ${ }^{136} \mathrm{Xe}$. High energyresolution cryogenic detectors such as $\mathrm{ZnSe}, \mathrm{ZnMoO}_{4}$ with pulse shape analyzes and/or scintillation signals [223, 224] may be used to search for the NH masses by $0 \nu \beta \beta$-decay experiments of ${ }^{82} \mathrm{Se}$ and ${ }^{100} \mathrm{Mo}$.

\subsection{Experimental studies of DBD matrix elements}

\subsubsection{Experimental probes for DBD matrix elements}

Nuclear matrix elements $\left(M_{\nu}^{0 \nu}\right)$ for $0 \nu \beta \beta$-decay are crucial for extracting the effective Majorana $\nu$ mass and other parameters, relevant to particle physics models neutrinoless double-beta decay of ${ }^{136} \mathrm{Xe}$ with an exposure of $32.5 \mathrm{~kg}$-yr and a background of $\approx 1.5 \times 10^{-3}$ $\mathrm{kg}^{-1} \mathrm{keV}^{-1} \mathrm{y}^{-1}$ in the $\pm \sigma$ region of interest. This implies a lower limit on the half life, $T_{1 / 2}^{0 \nu \beta \beta} \geq 1.6 \times 10^{25}$ y (90\% CL), corresponding to an effective Majorana mass of less than 140-380 meV, depending on the nuclear matrix element. 


\section{Nuclear $\tau \sigma$ responses for $v$ in $\beta \& \beta \beta$}
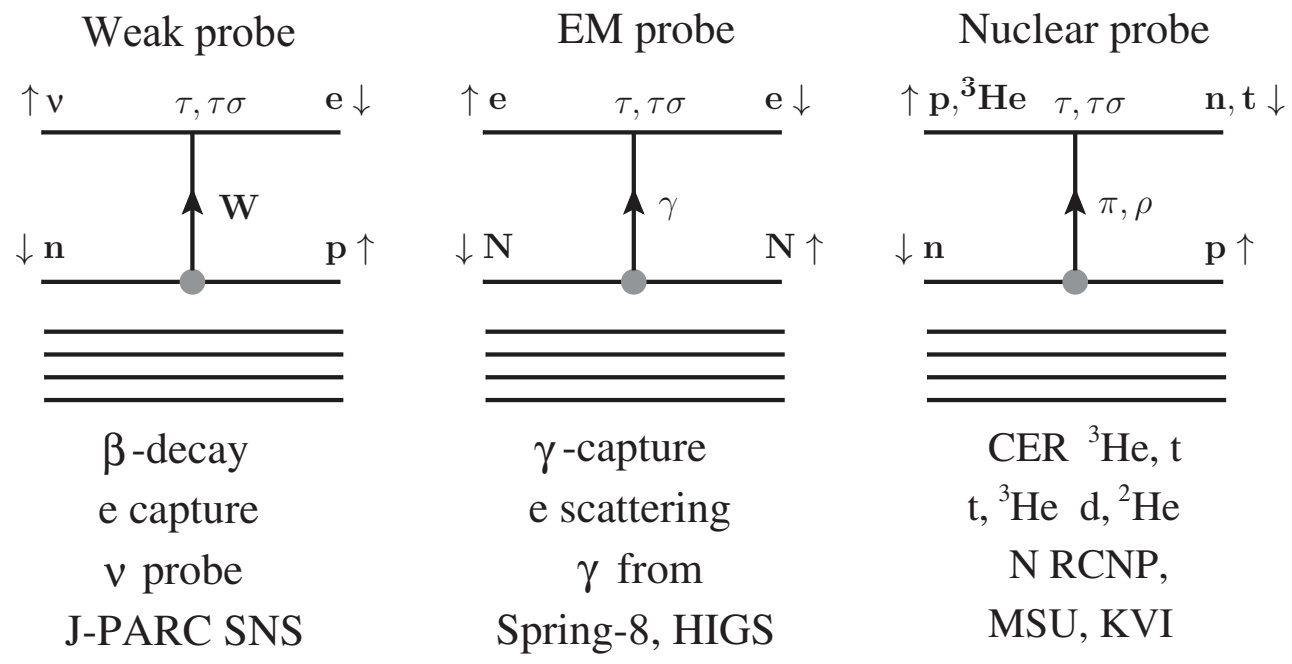

Figure 20: Nuclear spin isospin responses for weak interactions. They are studied by $\nu$ probes via weak interactions, EM $(\gamma)$ probes via EM interactions and by nuclear probes via strong interactions [49, 30, 132 ]

beyond the SM, from $0 \nu \beta \beta$ experiments, while nuclear matrix elements $\left(M^{2 \nu}\right)$ for $2 \nu \beta \beta$ decay can be derived experimentally from the observed $2 \nu \beta \beta$-decay half-lives. Extensive calculations of $M_{G T}^{2 \nu}$ and $M_{\nu}^{0 \nu}$ have been made in terms of QRPA, RQRPA, shell model, and so on, as given elsewhere in the theoretical sections.

Most $\beta \beta$ strengths are located in $\beta \beta$ (double Gamow-Teller) giant resonances, i.e., in the high-excitation region [261]. Thus the $\beta \beta$ matrix elements get very small in comparison with single particle estimates and are sensitive to nuclear structures, nuclear spin-isospin correlations, nuclear deformations, nuclear interactions, nuclear medium effects on the weak coupling constant $g_{A}$ and others. The theoretical evaluations for them are hard. Experimental studies of nuclear structures and nuclear interactions, which are relevant to $2 \nu \beta \beta$-decay and $0 \nu \beta \beta$-decay matrix elements, are very interesting to get reliable evaluations for them [49, 30, 261, 262].

Nuclear matrix elements of $M^{2 \nu_{G T}}$ and $M_{\nu}^{0 \nu}$ are expressed in terms of the successive single $\beta$ processes through intermediate $\left|J^{\pi}\right\rangle$ states. Among the intermediate states, low-lying single particle-hole states play dominant roles [261, 263]. The single $\beta$ matrix elements are given by spin-isospin responses for $Q_{T S L J}=\tau^{ \pm}\left[i^{L} r^{L} Y_{L} \times \sigma^{S}\right]$. They are studied experimentally using hadron, photon, and lepton probes as shown in Fig. 20,

\section{Lepton probes}

Nuclear weak responses for low-lying intermediate states are obtained from single $\beta$ decay rates and $\mathrm{EC}$ rates. However, they are limited to $\beta^{ \pm}$decays from the ground 
state in the intermediate nucleus. The decays are mostly allowed Gamow-Teller (GT) decays with $\tau^{ \pm} \sigma$, and are first forbidden decays with $\tau^{ \pm} i r Y_{1}$ and $\tau^{ \pm}\left[i r^{L} Y_{L} \times \sigma^{S}\right]$ in some nuclei.

Muon capture reactions of $\left(\mu^{-}, \nu_{\mu}\right)$ are used to get the $\beta^{+}$strengths in the intermediate nucleus [262, 264]. Excitation energies and angular momenta involved in this reaction are $E=0-50 \mathrm{MeV}$ and $0^{ \pm}, 1^{ \pm}$and $2^{ \pm}$. The capture cross section is quite large, and most muons are stopped and captured in the case of medium heavy nuclei. Then the $\tau^{+}$weak strength distribution in the intermediate nucleus is derived by measuring the decaying neutrons and $\gamma$-rays from excited states and radioactive isotopes produced by the muon capture.

One direct way to get the weak responses is to use $\nu$ beams. Since $\nu$ nuclear cross sections are as small as $\sigma=10^{-40 \sim 42} \mathrm{~cm}^{2}$, one needs high-flux $\nu$ beams and large detectors [49, 30, 132]. Low energy $\nu$ beams with $E \leq 100 \mathrm{MeV}$ can be obtained from pion decays. Intense pions are produced by nuclear interaction with $\mathrm{GeV}$ protons. Weak decays of stopped $\pi^{+}$give low energy neutrinos as

$$
\pi^{+} \rightarrow \mu^{+}+\nu_{\mu}, \quad \mu^{+} \rightarrow \nu_{e}+\bar{\nu}_{\mu}+e^{+},
$$

where $\nu_{\mu}$ and $\nu_{e}, \bar{\nu}_{\mu}$ are well separated by the decay time. Intense $1 \mathrm{GeV}$ protons from SNS at ORNL provides intense neutrinos around $10^{15}$ per second [265] and the J-PARC booster synchrotron with $3 \mathrm{GeV}$ protons produces neutrinos around $3 \times 10^{14}$ per second [266].

Photon probes

Weak responses for $\beta^{+}$decays are studies by using photo-nuclear $(\gamma, \mathrm{X})$ reactions through isobaric analogue states (IAS) as shown for the first time by $(\mathrm{p}, \gamma)$ reactions [267, 268]. The $\beta$ and $\gamma$ matrix elements are related as

$$
<f\left|g_{V} m^{\beta}\right| i>\approx \frac{g_{V}}{e}(2 T)^{1 / 2}<f\left|e m^{\gamma}\right| I A S>
$$

where $\left|I A S>=(2 T)^{-1 / 2} T^{-}\right| i>, T$ is the isospin of the parent nucleus and $m^{\beta}$ and $m^{\gamma}$ are analogous $\beta$ and $\gamma$ transition operators. Thus one can obtain the $\beta$ matrix element for $|i>\rightarrow| f>$ by observing the analogous $\gamma$ absorption $|f>\rightarrow| I A S>$ through the IAS of $\mid i>$, where $\mid f>$ and $\mid i>$ are the final state and the intermediate state in the $\beta \beta$-decay, as shown in Fig. 21. These photo-nuclear reactions through IAS are used to get the $\beta^{+}$matrix elements to excited states in the intermediate nucleus.

In medium heavy nuclei, IAS is located on the E1 giant resonance (GR) at the high excitation region. Accordingly IAS shows up as a sharp isobaric analogue resonance (IAR), and the photo-nuclear reaction includes IAR, GR, and the interference term as given by [269]

$$
\frac{d \sigma(\gamma, N)}{d \Omega}=k\left[\left|A(I)_{J^{\prime}}\right|^{2}+\Sigma_{J}\left|A(G)_{J}\right|^{2}+2 R e\left(A(I)_{J^{\prime}} A(G)_{J^{\prime}} e^{i \phi}\right)\right],
$$

where $A(I)_{J^{\prime}}, A(G)_{J^{\prime}}$ and $\phi$ are the IAR and GR amplitudes and the relative phase at IAR. Then one can get the phase of the matrix element from the interference. 


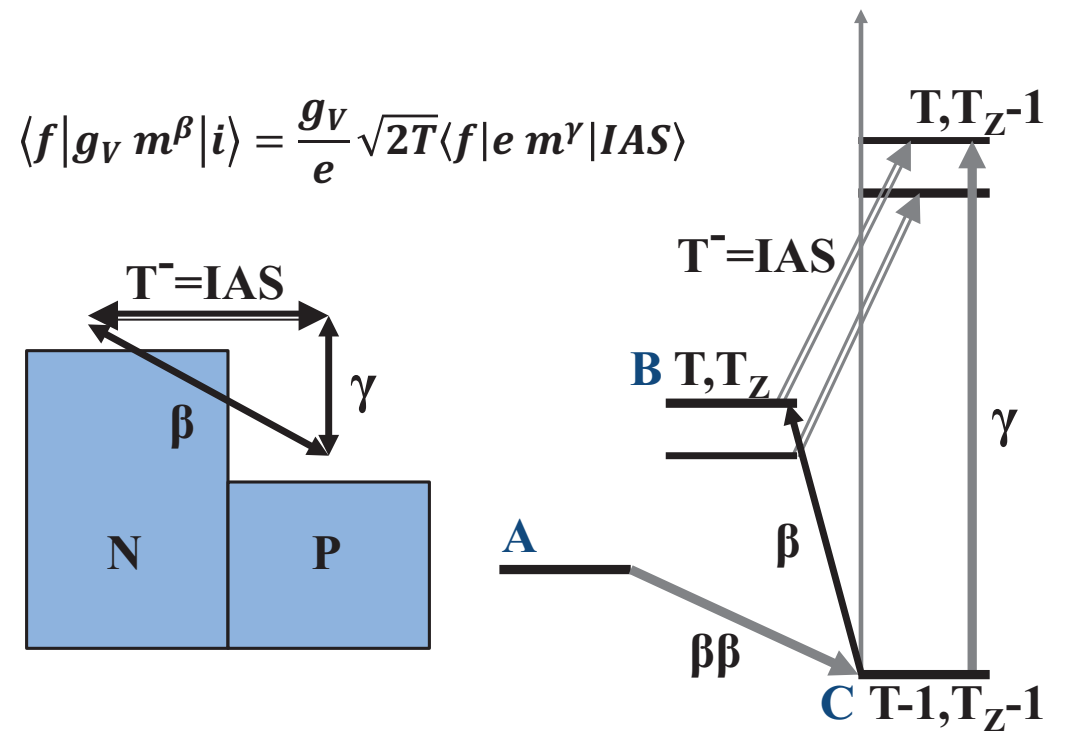

Figure 21: Level and transition schemes of $\beta \beta$, single $\beta^{+}$, and $\gamma$ via IAS. $\mathrm{T}^{-}$is the isospin lowering operator.

Laser electron photons, which are obtained from laser photons scattered off $\mathrm{GeV}$ electrons, are used for the photonuclear reaction. The polarization of the photon can be used to study E1 and M1 matrix elements separately [262]. HI $\gamma \mathrm{S}$ (High Intensity $\gamma$-ray Source), New SUBARU and other electron synchrotrons with $E_{e}=1-3 \mathrm{GeV}$ provide laser electron photons used for the photo-nuclear reaction.

Nucleon and nuclear probes

Nuclear charge exchange reactions with nuclear (hadron) probes are used to study nuclear spin-isospin responses. Charge exchange spin-nonflip reactions are used for vector weak responses, while charge exchange spin-flip reactions for axial-vector weak responses [30, 261]. Extensive studies of charge exchange reactions have been made to get $\mathrm{GT}\left(1^{+}\right)$responses with $\tau^{ \pm} \sigma$. The reactions studied are $(\mathrm{p}, \mathrm{n})(\mathrm{n}, \mathrm{p}),\left(\mathrm{d},{ }^{2} \mathrm{He}\right),\left({ }^{3} \mathrm{He}, \mathrm{t}\right)$ $\left(\mathrm{t},{ }^{3} \mathrm{He}\right)$, and $\left({ }^{7} \mathrm{Li},{ }^{7} \mathrm{Be}\right)$ at IUCF, KVI, MSU, RCNP, Triumf and others. Some charged particle experiments are in [270, 271, 272, 273, 274]. Medium-energy projectiles with $E_{i} / A=0.1-0.3 \mathrm{GeV}$ are used for studying $\tau \sigma$ responses because of the relatively large spin-isospin interaction $\left(V_{\tau \sigma}\right)$ and the small distortion interaction $\left(V_{0}\right)$ at the medium energy.

The charge exchange reaction with the medium-energy projectile is mainly due to the central isospin and spin-isospin interactions. Then the cross section with the transferred momentum $(q)$ and energy $(\omega)$ is given as

$$
\sigma_{\alpha}(q, \omega)=K\left(E_{i}, \omega\right) \exp \left(-\frac{1}{3} q^{2}\left\langle r^{2}\right\rangle\right) N_{\alpha}^{D}\left|J_{\alpha}\right|^{2} B(\alpha)
$$

where $K\left(E_{i}, \omega\right), N_{\alpha}^{D}, J_{\alpha}$, and $B(\alpha)$ are the kinematical factor, the nuclear distortion 
factor, the volume integral of the spin-isospin interaction, and the nuclear spin-isospin response, respectively. $\alpha$ denotes the isospin and spin channel; $\alpha=\mathrm{F}$ for isospin Fermi and $\alpha=$ GT for spin-isospin GT.

The cross section at $0 \operatorname{deg}$ for $q \approx 0$ and $\omega \approx 0$ is corrected for the kinematical and normalization factors, and is expressed as

$$
\frac{d \sigma_{\alpha}\left(0^{o}\right)}{d \Omega} \frac{1}{K\left(E_{i}, 0\right) N_{\alpha}^{D}}=\left|J_{\alpha}\right|^{2} B(\alpha), \quad \alpha=\mathrm{F}, \mathrm{GT} .
$$

The proportionality of the cross section at the forward angle of $\theta \sim 0$ deg to the $\tau \sigma$ response $B(\alpha)$ has been studied for charge exchange reactions with medium-energy light projectiles. In fact, the proportionality is good for spin-flip reactions with $B(G T) \geq 0.1$, but some deviation from the proportionality is found in the reactions with smaller $B(G T)$ due to contributions from tensor/non-central interactions.

Charge exchange $\left({ }^{3} \mathrm{He}, \mathrm{t}\right)$ reactions (ChER) relevant to the $\beta \beta$-decays were studied at RCNP by using the $420 \mathrm{MeV}^{3} \mathrm{He}$ beam and the high energy-resolution beam line and spectrometer system [270, 271, 275, 276, 277, 278, 279, 280, 281]. The beam stability and the beam line system have been improved to give the fantastic energy-resolution of $\Delta E / E \approx 5 \times 10^{-5}$ and $\Delta E \approx 25 \mathrm{keV}$, and ChERs have been studied for several $\beta \beta$ nuclei.

The GT strengths are mostly located at the GR resonances in the high excitation region, and accordingly, the strengths for the low-lying states are small. In cases of ${ }^{96} \mathrm{Zr},{ }^{100} \mathrm{Mo}$, and ${ }^{116} \mathrm{Cd}$, where valence neutrons and valence protons are in the different major shells, there is only one strong GT state (ground state) of $(\mathrm{g} 7 / 2)_{n}(\mathrm{~g} 9 / 2)_{p}$. Thus the $\left({ }^{3} \mathrm{He}, \mathrm{t}\right)$ reaction shows a strong GT strength only at the ground state. The high resolution $G T^{ \pm}$strength measurement can give significant insight into the details of the nuclear structure and can help to determine the $0 \nu \beta \beta$-decay NME less nuclear model dependent.

Nucleon transfer reactions were studied to get the occupation and vacancy probabilities of nucleons involved in double beta decays [282]. They are used to evaluate $\beta \beta$ matrix elements.

8.4.2. DBD matrix elements via low lying intermediate states In this subsestion, we briefly discuss experimental GT strengths for low-lying states and $2 \nu \beta \beta$ matrix elements [132, 30]. The matrix element $M^{2 \nu}$ is given approximately by a sum of products of two single GT matrix elements, $M_{G T}^{(+)}(m)$ from the direction of $\beta^{+}$and $M_{G T}^{(-)}(m)$ from $\beta^{-}$. It is expressed as $M^{2 \nu} \approx M_{G T}^{2 \nu}$, and

$$
M_{G T}^{2 \nu}=\sum_{m} \frac{M_{G T}^{(+)}(m) M_{G T}^{(-)}(m)}{Q_{\beta \beta} / 2+m_{e}+E_{x}\left(1_{m}^{+}\right)-E_{0}},
$$

Here, $\left(E_{x}\left(1_{m}^{+}\right)-E_{0}\right)$ is the energy difference between the $m^{\text {th }}$ intermediate $1^{+}$state and the initial ground state. $Q_{\beta \beta}$ is the $\mathrm{Q}$-value of the $\beta \beta$-decay.

The single GT matrix elements can be derived from charge exchange $\left({ }^{3} \mathrm{He}, \mathrm{t}\right)$, $\left(\mathrm{d},{ }^{2} \mathrm{He}\right)$ and other reactions and $\beta^{ \pm} /$EC-decay rates. Measurements of $G T^{ \pm}$strengths 
have been performed for $\mathrm{A}=100,116$ [270, 271], 48 [274, 276], 76 [277], $\mathrm{A}=96$ [272, 279], 116 [275], $\mathrm{A}=136$ [280] and 150 [281]. For $A=128$ and 130 the data are expected to be available soon.

The coherent sum of the weighted products of the measured $M_{G T}^{+}(m)$ and $M_{G T}^{(-)}(m)$ are in accord with the observed $M_{G T}^{2 \nu}[270,279]$. Actually it has been shown that the $2 \nu \beta \beta$ proceeds through low-lying (Fermi Surface) states, and not much through the GT giant resonance [132, 263], in agreements with observations by charge exchange reactions. The measured $2 \nu \beta \beta$ half-lives and the matrix elements[241] are given in Table 2.

SSD (Sigle state dominance) hypothesis [283. suggests that $2 \nu \beta \beta$-decay transitions, where the first $1^{+}$state of the intermediate nucleus is the ground state (e.g., ${ }^{100} \mathrm{Mo}$, ${ }^{116} \mathrm{Cd},{ }^{128} \mathrm{Te}$ ) are governed by the transition through that $J^{\pi}=1^{+}$state. The SSD hypothesis looks fine in case of nuclei like ${ }^{100} \mathrm{Mo}$, where there is only one $J^{\pi}=1^{+}$state.

Recently the nuclear matrix elements $M_{G T}^{2 \nu}$ are shown to be expressed in terms of single $\beta$ matrix elements via Fermi-surface quasi particle states (FSQP) [263, 284, 285].

$$
M_{G T}^{2 \nu}=\sum_{k} M(k)(\Delta(k))^{-1}, \quad M(k)=M_{G T}^{(+)}(k) M_{G T}^{(-)}(k),
$$

where the sum is over the FSQP (low-lying) states $(k)$ in the intermediate nucleus, $\Delta(k)$ is the energy denominator and the matrix elements of $M_{G T}^{(+)}(k)$ and $M_{G T}^{(-)}(k)$ are the experimental single GT matrix elements deduced from charge exchange reactions, $\beta$ decay rates and EC rates. Thus it includes no adjustable parameters. The possible deviation of $g_{A}$ from 1.26 is embedded in the observed matrix elements of $M_{G T}^{2 \nu}$ and similarly in $M_{G T}^{(+)}(k), M_{G T}^{(-)}(k)$.

In the quasi-particle representation, the experimental matrix elements are given by

$$
M_{G T}^{(-)}(k)=k_{A}^{e f f_{i}} m\left(j_{k}, J_{k}\right) P_{i}(k), \quad M_{G T}^{(+)}(k)=k_{A}^{e f f_{f}} m\left(j_{k}, J_{k}\right) P_{f}(k),
$$

where $m\left(j_{k} J_{n} k\right)$ is the single particle matrix element for the GT transition between single particle $j_{k}$ and $J_{k}$ states with $j_{k}$ and $J_{k}$ being the neutron and proton spins, and $P_{i}(k)=U_{p}\left(J_{k}\right) V_{n}\left(j_{k}\right)$ and $P_{f}(k)=V_{p}\left(J_{k}\right) U_{n}\left(j_{k}\right)$ are the pairing reduction factors for transitions from the initial ground state to $\mathrm{k}$-th intermediate state and from this state to final state, respectively. The effective coupling constants, $k_{A}^{e f f_{i}}$ and $k_{A}^{e f f_{f}}$, in unit of $g_{A}$, represent the nuclear core effects such as the spin-isospin correlations, the short-range correlations, and others, in addition to the quenching effect, while the nuclear surface (shell) effects are given by the pairing factors of $P_{i}(k)$ and $P_{f}(k)$. In fact values for $k_{A}^{e f f_{i}}$ and $k_{A}^{e f f_{f}}$ do not depend much on individual states, and thus one can evaluate the single $\beta$ matrix elements, if not available experimentally, by using the $k_{A}^{e f f_{i}}$ and $k_{A}^{e f f_{f}}$ for other states in neighboring nuclei and the caluculated values for $m\left(j_{k}, J_{k}\right) P_{i, f}(k)$.

As shown in Fig. 22, the observed $2 \nu \beta \beta$-decay matrix elements are well reproduced by the sum of the matrix elements through the low-lying FSQP $1^{+}$states in intermediate nucleus.

In general there are several low-lying $1^{+}$states, and thus $2 \nu \beta \beta$-decay proceeds not only through the lowest state but also other FSQP states. The product of the matrix 


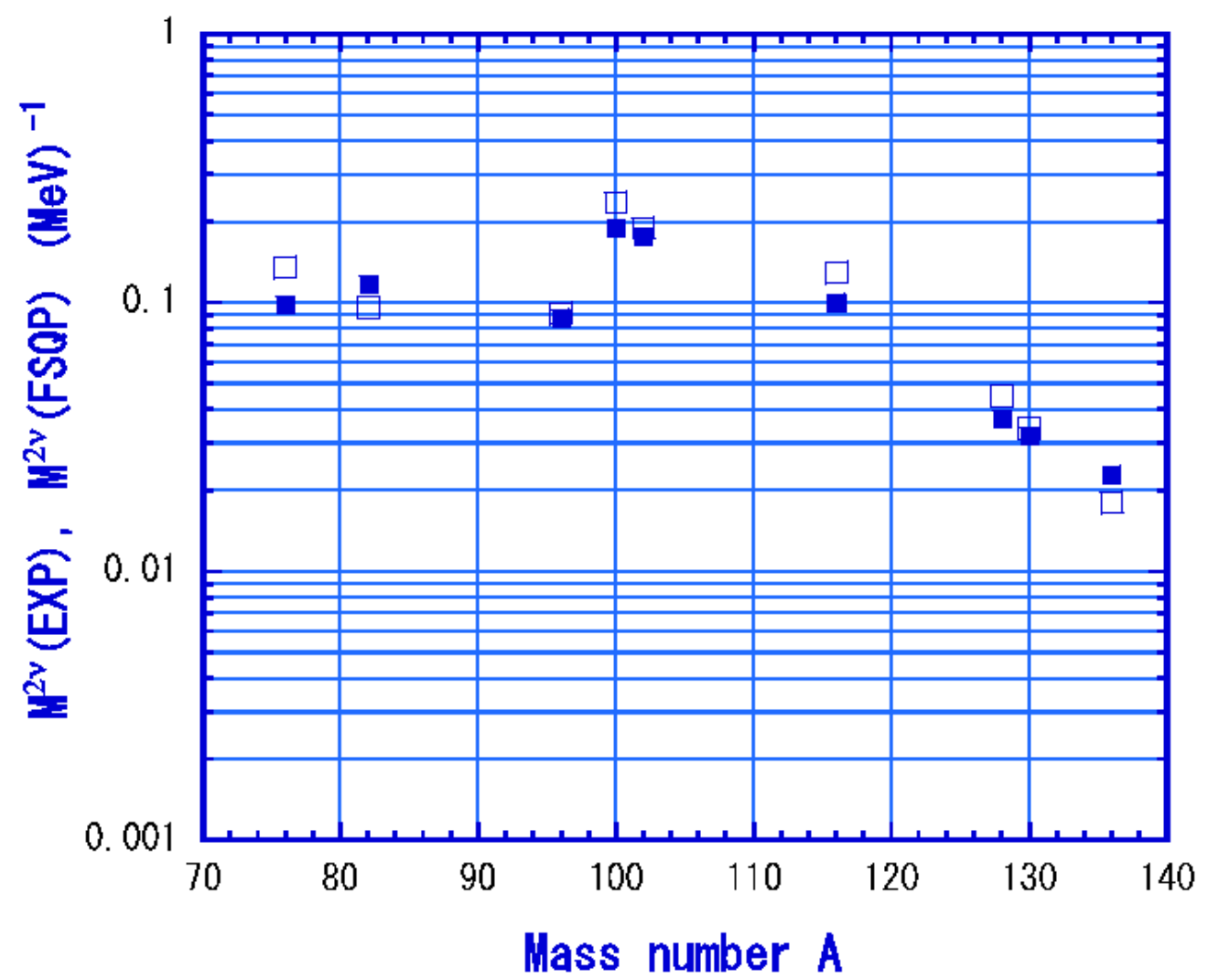

Figure 22: Nuclear matrix elements for $2 \nu \beta \beta$ decays . Open squares: observed values $\left(M^{2 \nu}\right)$, closed squares: FSQP values $(M=\Sigma M(k) / \Delta(k))$. The matrix elements for the excited $0^{+}$state in ${ }^{100} \mathrm{Ru}$ are plotted at $A=102$ [284, 285].

elements is $M_{G T}^{(+)}(k) M_{G T}^{(-)}(k)=k_{A}^{e f f_{i}} k_{A}^{e f f_{f}}\left(m\left(j_{k}, J_{k}\right)\right)^{2} P_{i}(k) P_{f}(k)$, which is positive. Thus contributions from the FSQP states are not cancelled with each other, but are constructive.

The $0 \nu \beta \beta$ transition operator is a two-body operator. It is shown that the matrix element is approximately given by the sum of the matrix elements for successive processes via intermediate states [30, 132]. Then the matrix elements for the successive $\beta$ transitions via low-lying intermediate states are derived from the single $\beta$ matrix elements as in case of the $2 \nu \beta \beta$ process.

Since the $0 \nu \beta \beta$-decay process is a virtual neutrino exchange between 2 nucleons in the nucleus, intermediate states with higher spins are involved. They are studied by measuring angular distributions of charge exchange reactions to intermediate $1^{ \pm}, 2^{ \pm}$, states and photo-excitations to IAS of the intermediate $1^{ \pm}$states 262 . Charge exchange reactions and photo-excitations of IAS are under progress by Muenster, MSU, NC, RCNP, and other groups to study nuclear structures relevant to $2 \nu \beta \beta$ and $0 \nu \beta \beta$ processes. 


\subsection{Two-neutrino double beta decay and bosonic neutrino}

Neutrinos may possibly violate the spin-statistics theorem, and hence obey Bose statistics or mixed statistics despite having spin half. A violation of the spin-statistics relation for neutrinos would lead to a number of observable effects in cosmology and astrophysics. In particular, bosonic neutrinos might compose all or a part of the cold cosmological dark matter (through bosonic condensate of neutrinos) and simultaneously provide some hot dark matter. A change of neutrino statistics would have an impact on the evolution of supernovae and on the spectra of supernova neutrinos. The idea of bosonic neutrinos has been proposed independently in Ref. [286], where cosmological and astrophysical consequences of this hypothesis have been studied.

If neutrinos obey at least partly the Bose-Einstein statistics the Pauli exclusion principle (PEP) is violated for neutrinos. The parameter $\sin ^{2} \chi$ can be introduce to characterize the bosonic (symmetric) fraction of the neutrino wave function [287]. A smooth change of $\sin ^{2} \chi$ from 0 to 1 transforms fermionic neutrinos into bosonic ones. The assumption of violation of the PEP leads to a number of fundamental problems which include loss of a positive definiteness of energy, violation of the CPT invariance, and possibly, of the Lorentz invariance as well as of the unitarity of S-matrix. No satisfactory and consistent mechanism of the Pauli exclusion principle violation has been proposed so far.

The lepton number conserving $2 \nu \beta \beta$-decay) can be used to study the statistical properties of neutrinos [287]. In the presence of bosonic neutrinos two contributions to the amplitude of the decay from diagram with permuted neutrino momenta have relative plus sign instead of minus in the Fermi-Dirac case.

The PEP violation strongly changes the rates of the $2 \nu \beta \beta$-decays and modifies the energy and angular distributions of the emitted electrons. The effect of bosonic neutrinos is different for transitions to $0^{+}$ground states and $2^{+}$excited states. In Fig. 23 the energy spectra of two electrons for different values of the bosonic-fraction $\sin ^{2} \chi$ is presented for the $2 \nu \beta \beta$-decay of ${ }^{100}$ Mo to ground state of final nucleus. With increase of $\sin ^{2} \chi$ the spectra shift to smaller energies. We note that substantial shift occurs only when $\sin ^{2} \chi$ is close to 1.0 Pure bosonic neutrinos are excluded by the present data. [287]. In the case of partly bosonic (or mixed-statistics) neutrinos the analysis of the existing data allows to put the conservative upper bound $\sin ^{2} \chi<0.6$ [287].

\section{Effective transition operators}

The subject of interest are the lepton number violating (LNV) parameters associated with the exchange of light and heavy Majorana neutrinos and with R-parity breaking SUSY mechanisms.

By assuming the dominance of a single mechanism determined by the LNV parameter $\eta_{\kappa}$ the inverse value of the $0 \nu \beta \beta$-decay half-life for a given isotope $(A, Z)$ 


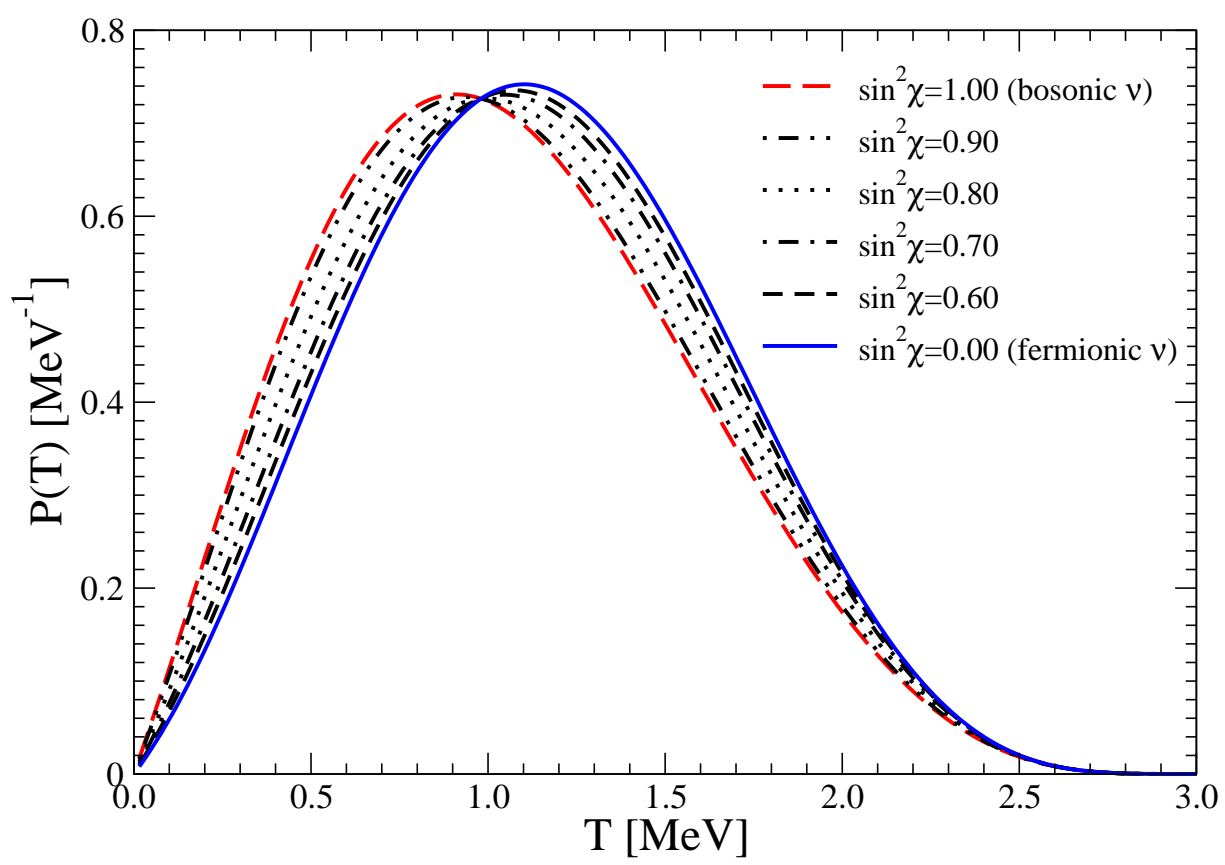

Figure 23: (Color online) The differential decay rates normalized to the total decay rate vs. the sum of the kinetic energy of outgoing electrons $T$ for $2 \nu \beta \beta$-decay of ${ }^{100} \mathrm{Mo}$ to the ground state of final nucleus. The results are presented for different values of the squared admixture $\sin ^{2} \chi$ of the bosonic component. The spectra have been calculated in the single state dominance approximation [288, 289].

can be written as

$$
\frac{1}{T_{1 / 2}^{0 \nu}}=\left|\eta_{\kappa}\right|^{2}\left|M_{\kappa}^{\prime 0 \nu}\right|^{2} G^{0 \nu}\left(E_{0}, Z\right) .
$$

Here, $G^{0 \nu}\left(E_{0}, Z\right)$ and $M_{\kappa}^{\prime 0 \nu}$ are, respectively, the known phase-space factor $\left(E_{0}\right.$ is the energy release) and the nuclear matrix element, which depends on the nuclear structure of the particular isotopes $(A, Z),(A, Z+1)$ and $(A, Z+2)$ under study.

The phase space factor $G^{0 \nu}\left(E_{0}, Z\right)$ includes fourth power of unquenched axial-vector coupling constant $g_{A}$ and the inverse square of the nuclear radius $R^{-2}$, compensated by the factor $R$ in $M^{\prime 0 \nu}$. The assumed value of the nuclear radius is $R=r_{0} A^{1 / 3}$ with $r_{0}=1.1 \mathrm{fm}$ or $r_{0}=1.2 \mathrm{fm}$ in different publications. The implicit radius and $g_{A}$ dependencies in $G^{0 \nu}\left(E_{0}, Z\right)$ and nuclear matrix element and the problem of the correct use of them were discussed in [290].

The nuclear matrix element $M^{\prime 0 \nu}$ is defined as

$$
M_{\kappa}^{\prime 0 \nu}=\left(\frac{g_{A}^{e f f}}{g_{A}}\right)^{2} M_{\kappa}^{0 \nu} .
$$

Here, $g_{A}^{e f f}$ is the quenched axial-vector coupling constant. This definition of $M_{\kappa}^{\prime 0 \nu}$ [291, 292] allows to display the effects of uncertainties in $g_{A}^{e f f}$ and to use the same phase factor $G^{0 \nu}\left(E_{0}, Z\right)$ when calculating the $0 \nu \beta \beta$-decay rate. 
Before we proceed with the discussion of the nuclear matrix elements we will summarize the various types of transition operators entering the neutrinoless double beta decay process. We recall that in the various particle models the lightest particle exchanged between the two nucleons participating in this process is either much lighter than the electron or much heavier than the proton. It is thus possible to separate the particle physics parameters from those of nuclear physics. Furthermore the nature of this exchanged particle dictates the form of the transition operators. The LNV parameters of interest together with corresponding nuclear matrix elements are presented briefly below.

\subsection{Transition operators resulting from light neutrino exchange}

In the case of light-neutrino mass mechanism of the $0 \nu \beta \beta$-decay we have

$$
\eta_{\nu}=\left|\frac{\left\langle m_{\nu}\right\rangle}{m_{e}}\right|^{2}
$$

The nuclear matrix element associated with the light Majorana-neutrino exchange $M_{\nu}^{0 \nu}$ consists of the Fermi (F), Gamow-Teller (GT) and tensor (T) parts as

$$
\begin{aligned}
M_{\nu}^{0 \nu} & =-\frac{M_{F}^{0 \nu}}{\left(g_{A}^{e f f}\right)^{2}}+M_{G T}^{0 \nu}-M_{T}^{0 \nu} \\
& =\left\langle 0_{i}^{+}\left|\sum_{k l} \tau_{k}^{+} \tau_{l}^{+}\left[-\frac{H_{F}\left(r_{k l}\right)}{\left(g_{A}^{e f f}\right)^{2}}+H_{G T}\left(r_{k l}\right) \sigma_{k l}-H_{T}\left(r_{k l}\right) S_{k l}\right]\right| 0_{f}^{+}\right\rangle .
\end{aligned}
$$

Here

$$
S_{k l}=3\left(\vec{\sigma}_{k} \cdot \hat{\mathbf{r}}_{k l}\right)\left(\vec{\sigma}_{l} \cdot \hat{\mathbf{r}}_{k l}\right)-\sigma_{k l}, \quad \sigma_{k l}=\vec{\sigma}_{k} \cdot \vec{\sigma}_{l}
$$

The radial parts of the exchange potentials are

$$
H_{F, G T, T}\left(r_{k l}\right)=\frac{2}{\pi} R \int_{0}^{\infty} \frac{j_{0,0,2}\left(q r_{k l}\right) h_{F, G T, T}\left(q^{2}\right) q}{q+\bar{E}} d q .
$$

where $R$ is the nuclear radius and $\bar{E}$ is the average energy of the virtual intermediate states used in the closure approximation. The closure approximation is adopted in the calculation of the NMEs relevant for $0 \nu \beta \beta$-decay with the exception of the QRPA. The functions $h_{F, G T, T}\left(q^{2}\right)$ are given by [293]

$$
\begin{aligned}
h_{F}\left(q^{2}\right)= & f_{V}^{2}\left(q^{2}\right), \\
h_{G T}\left(q^{2}\right)= & \frac{2}{3} f_{V}^{2}\left(q^{2}\right) \frac{\left(\mu_{p}-\mu_{n}\right)^{2}}{\left(g_{A}^{e f f}\right)^{2}} \frac{q^{2}}{4 m_{p}^{2}}+ \\
& f_{A}^{2}\left(q^{2}\right)\left(1-\frac{2}{3} \frac{q^{2}}{q^{2}+m_{\pi}^{2}}+\frac{1}{3} \frac{q^{4}}{\left(q^{2}+m_{\pi}^{2}\right)^{2}}\right), \\
h_{T}\left(q^{2}\right)= & \frac{1}{3} f_{V}^{2}\left(q^{2}\right) \frac{\left(\mu_{p}-\mu_{n}\right)^{2}}{\left(g_{A}^{e f f}\right)^{2}} \frac{q^{2}}{4 m_{p}^{2}}+ \\
& \frac{1}{3} f_{A}^{2}\left(q^{2}\right)\left(2 \frac{q^{2}}{\left(q^{2}+m_{\pi}^{2}\right)}-\frac{q^{4}}{\left(q^{2}+m_{\pi}^{2}\right)^{2}}\right) .
\end{aligned}
$$


For the normalized to unity vector and axial-vector form factors the usual dipole approximation is adopted: $f_{V}\left(q^{2}\right)=1 /\left(1+q^{2} / M_{V}^{2}\right)^{2}, f_{A}\left(q^{2}\right)=1 /\left(1+q^{2} / M_{A}^{2}\right)^{2} . M_{V}=$ $850 \mathrm{MeV}$, and $M_{A}=1086 \mathrm{MeV} . g_{A}=1.254$ is assumed and the difference in magnetic moments of proton and neutron is $\left(\mu_{p}-\mu_{n}\right)=4.71$.

The above definition of the $M_{\nu}^{0 \nu}$ includes contribution of the higher order terms of the nucleon current and for the induced pseudoscalar the Goldberger-Treiman PCAC relation, $g_{P}\left(q^{2}\right)=2 m_{p} g_{A}\left(q^{2}\right) /\left(q^{2}+m_{\pi}^{2}\right)$ was employed [101].

\subsection{Transition operators resulting the heavy neutrino exchange mechanism}

We assume that the neutrino mass spectrum include heavy Majorana states $N_{k}$ with masses $M_{k}$ much larger than the typical energy scale of the $0 \nu \beta \beta$-decay. These heavy states can mediate this process as the previous light neutrino exchange mechanism. The difference is that the neutrino propagators in this case can be contracted to points and, therefore, the corresponding effective transition operators are local unlike in the light neutrino exchange mechanism with long range internucleon interactions. The corresponding LNV parameters are $\eta_{N}^{L}$ and $\eta_{N}^{R}$.

Separating the Fermi (F), Gamow-Teller (GT) and the tensor (T) contributions we write down for the NME

$$
\begin{aligned}
\mathcal{M}_{N}^{0 \nu} & =-\frac{M_{F(N)}}{g_{A}^{2}}+M_{G T(N)}-M_{T(N)} \\
& =\left\langle 0_{i}^{+}\left|\sum_{k l} \tau_{k}^{+} \tau_{l}^{+}\left[\frac{H_{F}^{(N)}\left(r_{k l}\right)}{g_{A}^{2}}+H_{G T}^{(N)}\left(r_{k l}\right) \sigma_{k l}-H_{T}^{(N)}\left(r_{k l}\right) S_{k l}\right]\right| 0_{f}^{+}\right\rangle
\end{aligned}
$$

where $S_{k l}$ and $\sigma_{k l}$ are given in Eq. (144). The radial parts of the exchange potentials are

$$
H_{F, G T, T}^{(N)}\left(r_{k l}\right)=\frac{2}{\pi} \frac{R}{m_{p} m_{e}} \int_{0}^{\infty} j_{0,0,2}\left(q r_{k l}\right) h_{F, G T}\left(q^{2}\right) q^{2} d q .
$$

\subsection{Transition operators resulting from the R-parity breaking SUSY mechanism}

Assuming the dominance of gluino exchange, we obtain for the LNV parameter the following simplified expression

$$
\eta_{\lambda^{\prime}}=\frac{\pi \alpha_{s}}{6} \frac{\lambda_{111}^{\prime 2}}{G_{F}^{2} m_{\tilde{d}_{R}}^{4}} \frac{m_{p}}{m_{\tilde{g}}}\left[1+\left(\frac{m_{\tilde{d}_{R}}}{m_{\tilde{u}_{L}}}\right)^{2}\right]^{2} .
$$

Here, $G_{F}$ is the Fermi constant, $\alpha_{s}=g_{3}^{2} /(4 \pi)$ is $\mathrm{SU}(3)_{\text {c }}$ gauge coupling constant. $m_{\tilde{u}_{L}}$, $m_{\tilde{d}_{R}}$ and $m_{\tilde{g}}$ are masses of the u-squark, d-squark and gluino, respectively.

We should mention again that for this type of interactions the pion-exchange mechanism ( $1 \pi$ and $2 \pi$ ) discussed in subsection 7.2 dominates over the conventional two-nucleon mechanism. Thus, denoting the $0 \nu \beta \beta$-decay NME associated with gluino and neutralino exchange as $\mathcal{M}_{\lambda^{\prime}}^{0 \nu}$, we have [28, 93]

$$
\mathcal{M}_{\lambda^{\prime}}^{0 \nu}=c^{1 \pi}\left(M_{G T}^{1 \pi}-M_{T}^{1 \pi}\right)+c^{2 \pi}\left(M_{G T}^{2 \pi}-M_{T}^{2 \pi}\right)
$$


with

$$
c^{1 \pi}=-\frac{2}{9} \frac{\sqrt{2} f_{\pi} m_{\pi}^{4}}{m_{p}^{3} m_{e}\left(m_{u}+m_{d}\right)} \frac{g_{s} F_{P}}{g_{A}^{2}}, \quad c^{2 \pi}=\frac{1}{18} \frac{f_{\pi}^{2} m_{\pi}^{4}}{m_{p}^{3} m_{e}\left(m_{u}+m_{d}\right)^{2}} \frac{g_{s}^{2}}{g_{A}^{2}} .
$$

Here, $g_{S}$ and $F_{P}$ stand for the standard pion-nucleon coupling constant $\left(g_{s}=13.4\right)$ and the nucleon pseudoscalar constant (we take the bag model value $F_{P} \approx 4.41$ from Ref. [204]), respectively. $f_{\pi}=0.668 m_{\pi}$ and $m_{\pi}$ is the mass of pion. $m_{u}$ and $m_{d}$ denote current quark masses. The partial nuclear matrix elements of the $R_{p}$ SUSY mechanism for the $0 \nu \beta \beta$ process are:

$$
\begin{aligned}
& M_{G T}^{k \pi}=\left\langle 0_{f}^{+}\left|\sum_{k \neq l} \tau_{k}^{+} \tau_{l}^{+} H_{G T}^{k \pi}\left(r_{k l}\right) \sigma_{i} \cdot \sigma_{j}\right| 0_{i}^{+}\right\rangle, \\
& M_{T}^{k \pi}=\left\langle 0_{f}^{+}\left|\sum_{k \neq l} \tau_{k}^{+} \tau_{l}^{+} H_{T}^{k \pi}\left(r_{k l}\right) S_{k l}\right| 0_{i}^{+}\right\rangle
\end{aligned}
$$

with

$$
\begin{aligned}
& H_{G T, T}^{1 \pi}\left(r_{k l}\right)=-\frac{2}{\pi} R \int_{0}^{\infty} j_{0,2}\left(q r_{k l}\right) \frac{q^{4} / m_{\pi}^{4}}{1+q^{2} / m_{\pi}^{2}} f_{A}^{2}\left(q^{2}\right) d q \\
& H_{G T, T}^{2 \pi}\left(r_{k l}\right)=-\frac{4}{\pi} R \int_{0,2}^{\infty} j_{0,2}\left(q r_{k l}\right) \frac{q^{4} / m_{\pi}^{4}}{\left(1+q^{2} / m_{\pi}^{2}\right)^{2}} f_{A}^{2}\left(q^{2}\right) d q .
\end{aligned}
$$

The two-nucleon exchange potentials are expressed in momentum space as the momentum dependence of normalized to unity nucleon formfactors $\left(f_{A}\left(q^{2}\right)\right)$ is taken into account.

\subsection{Transition operators resulting from the squark-neutrino mechanism}

In the case of squark-neutrino mechanism [202] due to the chiral structure of the R-parity breaking $\left(\not R_{p}\right)$ SUSY interactions, the amplitude of $0 \nu \beta \beta$-decay does not vanish in the limit of zero neutrino mass unlike the ordinary Majorana neutrino exchange mechanism proportional to the light neutrino mass. Instead, the squark-neutrino mechanism is roughly proportional to the momentum of the virtual neutrino which is of the order of the Fermi momentum of the nucleons inside of nucleus $p_{F} \approx 100 \mathrm{MeV}$. This is a manifestation of the fact that the LNV necessary for $0 \nu \beta \beta$-decay is supplied by the $\not R_{p}$ SUSY interactions instead of the Majorana neutrino mass term and therefore this mechanism is not suppressed by the small neutrino mass. The corresponding SUSY LNV parameter is defined as

$$
\eta_{\tilde{q}}=\sum_{k} \frac{\lambda_{11 k}^{\prime} \lambda_{1 k 1}^{\prime}}{2 \sqrt{2} G_{F}} \sin 2 \theta_{(k)}^{d}\left(\frac{1}{m_{\tilde{d}_{1}(k)}^{2}}-\frac{1}{m_{\tilde{d}_{2}(k)}^{2}}\right) .
$$

Here we use the notation $d_{(k)}=d, s, b$. This LNV parameter vanishes in the absence of $\tilde{q}_{L}-\tilde{q}_{R}-$ mixing when $\theta^{d}=0$.

At the hadron level we assume dominance of the pion-exchange mode. Then, the nuclear matrix element associated with squark-neutrino mechanism can be written as a 
sum of GT ad tensor contributions 202

$$
\mathcal{M}_{\tilde{q}}^{0 \nu}=M_{G T(\tilde{q})}-M_{T(\tilde{q})} .
$$

The exchange potentials are given by

$$
H_{G T, T}^{(\tilde{q})}\left(r_{k l}\right)=\frac{2}{\pi} R \int_{0}^{\infty} \frac{j_{0,2}\left(q r_{k l}\right) h^{\tilde{q}}\left(q^{2}\right) q^{2}}{q(q+\bar{E})} d q
$$

with

$$
h^{\tilde{q}}\left(q^{2}\right)=-\frac{1}{6} f_{A}^{2}\left(q^{2}\right) \frac{m_{\pi}^{4}}{m_{e}\left(m_{u}+m_{d}\right)} \frac{q^{2}}{\left(q^{2}+m_{\pi}^{2}\right)^{2}} .
$$

\section{0. $0 \nu \beta \beta$-decay nuclear matrix elements}

Interpreting existing results as a measurement of $\left|\left\langle m_{\nu}\right\rangle\right|$ and planning new experiments depends crucially on the knowledge of the corresponding nuclear matrix elements (NMEs) that govern the decay rate. The NMEs for $0 \nu \beta \beta$-decay must be evaluated using tools of nuclear structure theory. There are no observables that could be directly linked to the magnitude of $0 \nu \beta \beta$-decay nuclear matrix elements and, thus, could be used to determine them in an essentially model independent way. A reliable calculation of NMEs will be of help in predicting which are the most favorable nuclides to be employed for $0 \nu \beta \beta$-decay searches.

The evaluation of the nuclear matrix elements can be separated in two steps.

- The evaluation of the transition matrix elements between the two interacting particles (two body ME).

Each particle is assumed to occupy a set of single particle states, determined by the assumed model. The spin as well the orbital structure of the operator as it has been discussed in sections 5 and 6 . The operators discussed in section 5 are long ranged, except when the intermediate neutrino is heavy leading to short range transition operators. In section 6, except for the case of Eq. (110), all operators are short ranged. The way of dealing with the short range operators has been discussed in section 7. Taking all these into account the effective transition operators have been constructed in section 9 .

- The second step involves the construction of the many body wave functions.

One needs the wave functions of the initial and final nuclear systems. If closure is not employed, as in the case of QRPA, one also needs the wave functions of all the virtual (intermediate states) allowed by the assumed nuclear model. Some many body features arising from the nuclear medium are: i) the renormalization effects on the $g_{A}$ coupling and the modification of the nucleon currents, which have already been discussed in section 9 and ii) the short range correlations, which will be discussed below. The main techniques of the construction of the many body wave functions will be reviewed in this section. We remind the reader that in some cases information about the nuclear ME can be extracted from experiments (see 8.4). 
The calculation of the $0 \nu \beta \beta$-decay NMEs is a difficult problem because ground and many excited states (if closure approximation is not adopted) of open-shell nuclei with complicated nuclear structure have to be considered. In the last few years the reliability of the calculations has greatly improved. Five different many-body approximate methods have been applied for the calculation of the $0 \nu \beta \beta$-decay NME:

(i) The Interacting Shell Model (ISM) [236, 294, 295, 296, 297].

The ISM allows to consider only a limited number of orbits close to the Fermi level, but all possible correlations within the space are included. Proton-proton, neutronneutron and proton-neutron (isovector and isoscalar) pairing correlations in the valence space are treated exactly. Proton and neutron numbers are conserved and angular momentum conservation is preserved. Multiple correlations are properly described in the laboratory frame. The effective interactions are constructed starting from monopole corrected G matrices, which are further adjusted to describe sets of experimental energy levels. The Strasbourg-Madrid codes can deal with problems involving basis of $10^{11}$ Slater determinants, using relatively modest computational resources. A good spectroscopy for parent and daughter nuclei is achieved. Due to the significant progress in shell-model configuration mixing approaches, there are now calculations performed with these methods for several nuclei.

(ii) Quasiparticle Random Phase Approximation (QRPA) [115, 116].

The QRPA has the advantage of large valence space but is not able to comprise all the possible configurations. Usually, single particle states in the Wood-Saxon potential are considered. One is able to include to each orbit in the QRPA model space also the spin-orbit partner, which guarantees that the Ikeda sum rule is fulfilled. This is crucial to describe correctly the Gamow-Teller strength. The proton-proton and neutron-neutron pairings are considered. They are treated in the BCS approximation. Thus, proton and neutron numbers are not exactly conserved. The many-body correlations are treated at the RPA level within the quasiboson approximation. Two-body G-matrix elements, derived from realistic one-boson exchange potentials within the Brueckner theory, are used for the determination of nuclear wave functions.

(iii) Interacting Boson Model (IBM) [130].

In the IBM the low lying states of the nucleus are modeled in terms of bosons. The bosons are in either $\mathrm{L}=0$ ( $\mathrm{s}$ boson) or $\mathrm{L}=2$ ( $\mathrm{d}$ boson) states. Thus, one is restricted to $0^{+}$and $2^{+}$neutron pairs transferring into two protons. The bosons interact through one- and two-body forces giving rise to bosonic wave functions.

(iv) The Projected Hartree-Fock-Bogoliubov Method (PHFB) [129].

In the PHFB wave functions of good particle number and angular momentum are obtained by projection on the axially symmetric intrinsic HFB states. In applications to the calculation of the $0 \nu \beta \beta$-decay NMEs the nuclear Hamiltonian 
was restricted only to quadrupole interaction. The PHFB is restricted in its scope. With a real Bogoliubov transformation without parity mixing one can describe only neutron pairs with even angular momentum and positive parity.

(v) The Energy Density Functional Method (EDF) [131.

The EDF is considered to be an improvement with respect to the PHFB. The density functional methods based on the Gogny functional are taken into account. The particle number and angular momentum projection for mother and daughter nuclei is performed and configuration mixing within the generating coordinate method is included. A large single particle basis (11 major oscillator shells) is considered. Results are obtained for all nuclei of experimental interest.

The differences among the listed methods of NME calculations for the $0 \nu \beta \beta$-decay are due to the following reasons:

(i) The mean field is used in different ways. As a result, single particle occupancies of individual orbits of various methods differ significantly from each other [298].

(ii) The residual interactions are of various origin and renormalized in different ways.

(iii) Various sizes of the model space are taken into account.

(iv) Different many-body approximations are used in the diagonalization of the nuclear Hamiltonian.

Each of the applied methods has some advantages and drawbacks, whose effect in the values of the NME can be sometimes explored. The advantage of the ISM calculations is their full treatment of the nuclear correlations, which tends to diminish the NMEs. On the contrary, the QRPA, the EDF, and the IBM underestimate the multipole correlations in different ways and tend to overestimate the NMEs. The drawback of the ISM the limited number of orbits in the valence space and as a consequence the violation of Ikeda sum rule and underestimation of the NMEs.

In Table 3, recent results of the different methods are summarized. The presented numbers have been obtained with the unquenched value of the axial coupling constant $\left(g_{A}^{\text {eff }}=g_{A}=1.25\right)$, Miller-Spencer Jastrow short-range correlations [299] (the EDF values are multiplied by 0.80 in order to account for the difference between the unitary correlation operator method (UCOM) and the Jastrow approach [293]), the same nucleon dipole form-factors, higher order corrections to the nucleon current and the nuclear radius $R=r_{0} A^{1 / 3}$, with $r_{0}=1.2 \mathrm{fm}$ (the QRPA values [291, 292] for $r_{0}=1.1$ $\mathrm{fm}$ are rescaled with the factor 1.2/1.1). Thus, the discrepancies among the results of different approaches are solely related to the approximations on which a given nuclear many-body method is based.

From Table 3 we can make the following conclusions:

(i) The ISM values of NMEs, with the exception of the NME for the double magic nucleus ${ }^{48} \mathrm{Ca}$, practically do not depend on the nucleus. They are significantly smaller, by about a factor 2-3, when compared with NMEs of other approaches.

I A modern value of the axial-vector coupling constant is $g_{A}=1.269$. We note that in the referred calculations of the $0 \nu \beta \beta$-decay NMEs the previously accepted value $g_{A}^{e f f}=g_{A}=1.25$ was assumed. 
Table 3: The NME of the $0 \nu \beta \beta$-decay $M_{\nu}^{0 \nu}$ calculated in the framework of different approaches: interacting shell model (ISM) [236, 297], quasiparticle random phase approximation (QRPA) [184, 292, 300, 301, 302], projected Hartree-Fock Bogoliubov approach (PHFB, PQQ2 parametrization) ) [129], energy density functional method (EDF) 131 and interacting boson model (IBM) 130. QRPA(TBC) and QRPA(J) denote QRPA results of Tuebingen-Bratislava-Caltech (TBC) and Jyvaskyla (J) groups, respectively. The Miller-Spencer Jastrow two-nucleon short-range correlations are taken into account. The EDF results are multiplied by 0.80 in order to account for the difference between UCOM and Jastrow [293]. $g_{A}^{e f f}=g_{A}=1.25$ and $R=1.2 \mathrm{fm} A^{1 / 3}$ are assumed.

\begin{tabular}{|c|c|c|c|c|c|c|}
\hline \multirow[t]{3}{*}{ Transition } & \multicolumn{6}{|c|}{$M_{\nu}^{0 \nu}$} \\
\hline & $\overline{\mathrm{ISM}}$ & QRPA (TBC) & $\overline{\mathrm{QRPA}(\mathrm{J})}$ & IBM-2 & PHFB & $\overline{\mathrm{EDF}}$ \\
\hline & 236, 297] & [292, 184 & $300,301,302$ & 130 & 129 & 131 \\
\hline${ }^{48} \mathrm{Ca} \rightarrow{ }^{48} \mathrm{Ti}$ & $0.61,0.57$ & & & & & 1.91 \\
\hline${ }^{76} \mathrm{Ge} \rightarrow{ }^{72} \mathrm{Se}$ & 2.30 & 4.92 & 4.72 & 5.47 & & 3.70 \\
\hline${ }^{82} \mathrm{Se} \rightarrow{ }^{82} \mathrm{Kr}$ & 2.18 & 4.39 & 2.77 & 4.41 & & 3.39 \\
\hline${ }^{96} \mathrm{Zr} \rightarrow{ }^{96} \mathrm{Mo}$ & & 1.22 & 2.45 & & 2.78 & 4.54 \\
\hline${ }^{100} \mathrm{Mo} \rightarrow{ }^{100} \mathrm{Ru}$ & & 3.64 & 2.91 & 3.73 & 6.55 & 4.08 \\
\hline${ }^{110} \mathrm{Pd} \rightarrow{ }^{110} \mathrm{Cd}$ & & & 3.86 & & & \\
\hline${ }^{116} \mathrm{Cd} \rightarrow{ }^{116} \mathrm{Sn}$ & & 2.99 & 3.17 & & & 3.80 \\
\hline${ }^{124} \mathrm{Sn} \rightarrow{ }^{124} \mathrm{Te}$ & 2.10 & & 2.65 & & & 3.87 \\
\hline${ }^{128} \mathrm{Te} \rightarrow{ }^{128} \mathrm{Xe}$ & 2.34 & 3.97 & 3.56 & 4.52 & 3.89 & 3.30 \\
\hline${ }^{130} \mathrm{Te} \rightarrow{ }^{130} \mathrm{Xe}$ & 2.12 & 3.56 & 3.28 & 4.06 & 4.36 & 4.12 \\
\hline${ }^{136} \mathrm{Xe} \rightarrow{ }^{136} \mathrm{Ba}$ & 1.76 & 2.30 & 2.54 & & & 3.38 \\
\hline${ }^{150} \mathrm{Nd} \rightarrow{ }^{150} \mathrm{Sm}$ & & 3.16 & & 2.32 & 3.16 & 1.37 \\
\hline
\end{tabular}

(ii) The largest values of NME are obtained in the IBM $\left({ }^{76} \mathrm{Ge}\right.$ and $\left.{ }^{128} \mathrm{Te}\right), \mathrm{PHFB}\left({ }^{100} \mathrm{Mo}\right.$, ${ }^{130} \mathrm{Te}$ and $\left.{ }^{150} \mathrm{Nd}\right)$, QRPA $\left({ }^{150} \mathrm{Nd}\right)$ and $\mathrm{EDF}\left({ }^{48} \mathrm{Ca},{ }^{96} \mathrm{Zr},{ }^{116} \mathrm{Cd},{ }^{124} \mathrm{Sn}\right.$ and $\left.{ }^{136} \mathrm{Xe}\right)$ approaches.

(iii) NMEs obtained by the QRPA(TBC) and IBM methods are in a good agreement (with the exception of ${ }^{150} \mathrm{Nd}$ ).

(iv) In the case of ${ }^{130} \mathrm{Te}$ all discussed methods, with the exception of the ISM, give practically the same result.

(v) The disagreement between IBM-2 and ISM is particularly troublesome, because IBM-2 is a truncation of the shell-model space to the $S$ and $D$ pair space and, in the limit of spherical nuclei, IBM-2 and ISM should produce the same results.

(vi) The disagreement between the $\mathrm{QRPA}(\mathrm{TBC})$ and $\mathrm{QRPA}(\mathrm{J})$ results is not large but it needs to be clarified.

Comparing $0 \nu \beta \beta$-decay nuclear matrix elements calculated by different methods gives some insight in the advantages or disadvantages of different candidate nuclei. 


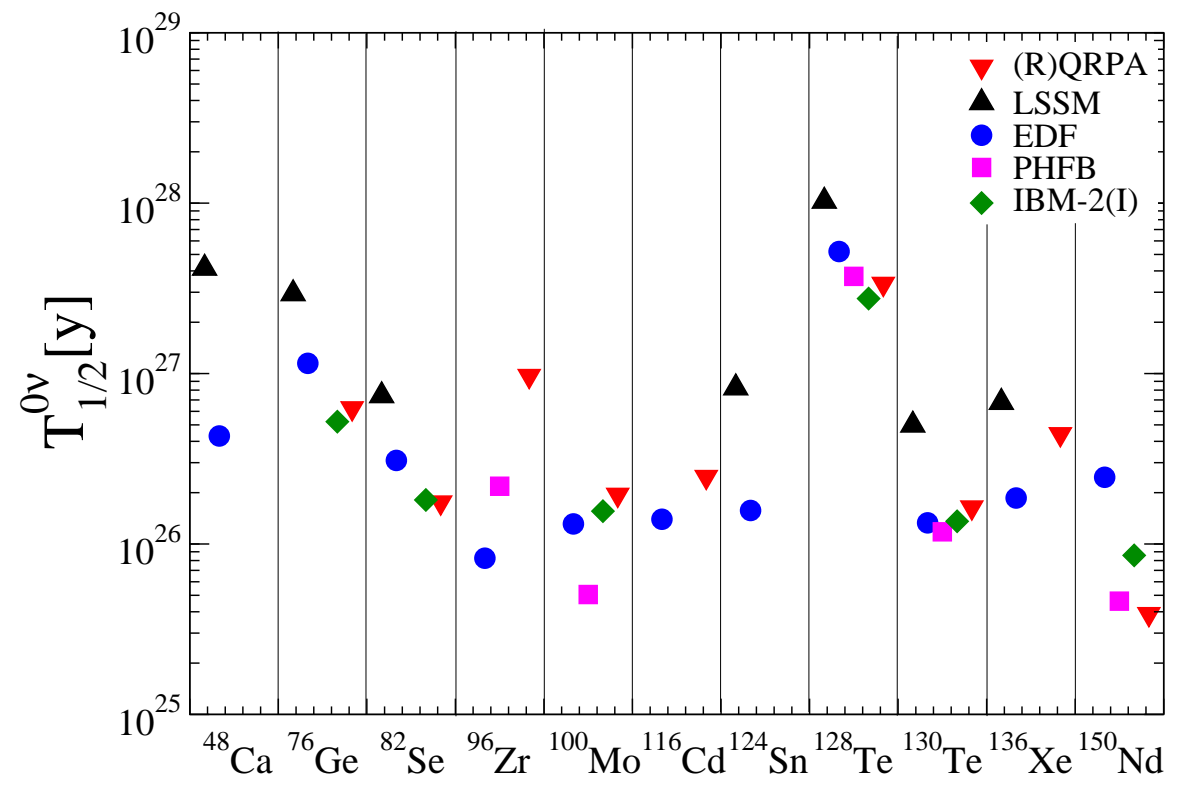

Figure 24: (Color online.) The $0 \nu \beta \beta$-decay half-lives of nuclei of experimental interest for $\left|\left\langle m_{\nu}\right\rangle\right|=50 \mathrm{meV}$ and NMEs of different approaches. The Miller-Spencer Jastrow two-nucleon short-range correlations are considered. The axial-vector coupling constant $g_{A}$ is assumed to be 1.25 .

However, matrix elements are not quite the only relevant quantities (see section 8 for the nuclear sensitivity factor). Experimentally, half-lives are measured or constrained, and the effective Majorana neutrino mass $\left\langle m_{\nu}\right\rangle$ is the ultimate goal. For $\left|\left\langle m_{\nu}\right\rangle\right|$ equal to $50 \mathrm{meV}$ the calculated half-lives for double $\beta$-decaying nuclei of interest are presented in Fig. 24. We see that the spread of half-lives for given isotope is up to the factor of 4-5.

It is worth to noticing that due to the theoretical efforts made over the last years the disagreement among different NMEs is now much less severe than it was about a decade before. Nevertheless the present-day situation with the calculation of $0 \nu \beta \beta$-decay NMEs can not be considered as completely satisfactory. Further progress is required and it is believed that the situation will be improved with time. Accurate determination of the NMEs, and a realistic estimate of their uncertainty, is of great importance. Nuclear matrix elements need to be evaluated with uncertainty of less than $30 \%$ to establish the neutrino mass spectrum and $\mathrm{CP}$ violating phases of the neutrino mixing

\subsection{Uncertainties in calculated NMEs}

The improvement of the calculation of the $0 \nu \beta \beta$-decay NMEs is a very important and challenging problem. The uncertainty associated with the calculation of the $0 \nu \beta \beta$ decay NMEs can be diminished by suitably chosen nuclear probes. Complementary experimental information from related processes like charge-exchange and particle transfer reactions, muon capture and charged current (anti)neutrino-nucleus reactions is 
very relevant. A direct confrontation of nuclear structure models with data from these processes improve quality of nuclear structure models (see section 8). The constrained parameter space of nuclear models is a promising way to reduce uncertainty in the calculated $0 \nu \beta \beta$-decay NMEs.

A steady progress in nuclear structure approaches is gradually leading to a better understanding and to a reduction of the differences among their results. However, even in the most refined approaches, the estimates of $M_{\nu}^{0 \nu}$ remain affected by various uncertainties, whose reduction is of great importance.

i) QRPA calculation of NMEs.

Due to its simplicity the QRPA is a popular technique to calculate the $0 \nu \beta \beta$-decay NMEs. One of the most important factors of the QRPA calculation of the $0 \nu \beta \beta$-decay NMEs is the way the particle-particle strength of the nuclear Hamiltonian $g_{p p}$ is fixed. The Tuebingen-Bratislava-Caltech (TBC) group has shown that by adjusting $g_{p p}$ to the $2 \nu \beta \beta$-decay rates the uncertainty associated with variations in QRPA calculations of the $0 \nu \beta \beta$-decay NMEs can be significantly eliminated [291, 292, 293, 303]. In particular, the results obtained in this way are essentially independent of the size of the basis, the form of different realistic nucleon-nucleon potentials, or on whether QRPA or renormalized QRPA (take into account Pauli exclusion principle) is used. This new way of fixing parameter space was criticized by the Jyvaskyla group in series of papers maintaining the role of single $\beta$-transitions. It was claimed that careful study of single $\beta$ and $2 \nu \beta \beta$ observables points to serious shortcomings of adopted procedure [304, 305]. These objections were refuted in [291, 292]. In the recent publications [300, 301, 302] also the Jyvaskyla group adopted the procedure of fixing of $g_{p p}$ proposed by the TBC group [303.

Usually, two variants of the QRPA are considered. The standard QRPA, which is based on the quasiboson approximation, and the renormalized QRPA (RQRPA) [127, 174, 291], which takes into account the Pauli exclusion principle. Further improvement is achieved within the self-consistent QRPA (SRQRPA) [298, 306, 307] by conserving the mean particle number in the correlated ground state. The restoration of Pauli exclusion principle and of particle number conservation lead to a reduction of the $0 \nu \beta \beta$-decay NMEs [174, 291, 292, 298].

There is some controversy about the importance of the tensor $M_{T}$ contribution to $M_{\nu}^{0 \nu}$. According to the ISM [236] the tensor term is small, a fact understood by the small model space adopted. The Jyvaskyla group performing the calculation within the QRPA claims that $M_{T}$ is negligible [300, 301]. $M_{T}$ was neglected in the PHFB [129] and EDF [131] calculations of the $0 \nu \beta \beta$-decay NMEs. Contrary, results of the IBM-2 [130] and the QRPA(TBC) [101] calculations show that $M_{T}$ can not be neglected and its absolute value can be up to $10 \%$ of $M_{\nu}^{0 \nu}$.

\section{ii) The closure approximation}

The $0 \nu \beta \beta$-decay matrix elements are usually calculated using the closure approximation for intermediate nuclear states. Within this approximation energies of intermediate states $\left(E_{n}-E_{i}\right)$ are replaced by an average value $\bar{E} \approx 10 \mathrm{MeV}$, and the sum 
over intermediate states is taken by closure, $\sum_{n}|n><n|=1$. This simplifies the numerical calculation drastically. The calculations with exact treatment of the energies of the intermediate nucleus were achieved within the QRPA-like methods [174, 291, 292, 293, 303]. The effect of the closure approximation was studied in detail in [308]. It was found that the differences in nuclear matrix elements are within $10 \%$. The the dependence of the NMEs on the average energy of the intermediate states $\bar{E}$ was studied within the nuclear shell model. By varying $\bar{E}$ from 2.5 to $12.5 \mathrm{MeV}$ the variation in the NME was obtained to be less than 5\% [297].

iii) The two-nucleon short range correlations and finite nucleon size.

The physics of finite nucleon size (FNS) and two-nucleon short-range correlations (SRC) is different. Both reduce magnitude of the $0 \nu \beta \beta$-decay NME by competing each with other. The importance each of them depends on the type of SRC and involved formfactor parameters.

The FNS is taken into account via momentum dependence of the nucleon formfactors. For the vector, weak-magnetism (axial-vector) the usual dipole approximation with cut-off parameter $M_{V}=850 \mathrm{MeV}\left(M_{A}=1086 \mathrm{MeV}\right)$, which comes come from electron scattering experiments (neutrino charged-current scattering experiments), is considered. The form-factors suppress high-momentum exchange. We note that in the limit of point-like nucleon $\left(M_{V, A} \rightarrow \infty\right)$ the weak magnetism contribution to the $0 \nu \beta \beta$-decay would be divergent.

The SRCs are included via the correlation function $\mathrm{f}(\mathrm{r})$, that modifies the relative two-nucleon wave functions at short distances:

$$
\Psi_{n l}(r) \rightarrow[1+f(r)] \Psi_{n l}(r),
$$

where $f(r)$ can be parametrized as [174]

$$
f(r)=-c e^{-a r^{2}}\left(1-b r^{2}\right) .
$$

Previously, Miller-Spencer Jastrow SRC ( $\left.a=1.1 \mathrm{fm}^{-2}, b=0.68 \mathrm{fm}^{-2}, c=1.0\right)$ have been added into the involved two-body transition matrix elements, changing two neutrons into two protons, to achieve healing of the correlated wave functions. A suppression of $M_{\nu}^{0 \nu}$ by $20 \%$ was found [130, 174, 236, 297]. However, recent work has questioned this prescription [174, 300, 301].

The two-nucleon short range correlations were studied within the coupled clusters method (CCM) in [174]. The Jastrow function fit for $\mathrm{T}=1$ channel reported for Argonne V18 and Bonn-CD NN interactions set of parameters $\left(a=1.59 \mathrm{fm}^{-2}, b=1.45 \mathrm{fm}^{-2}\right.$, $c=0.92)$ and $\left(a=1.52 \mathrm{fm}^{-2}, b=1.88 \mathrm{fm}^{-2}, c=0.46\right)$, respectively [174]. These correlation functions were confirmed by exploiting a construction of an effective shell model operator for $0 \nu \beta \beta$-decay of ${ }^{82}$ Se [309]. The notable differences between the results calculated with Miller-Spencer Jastrow and CCM SRC are about of 20\%-30\% [174, 297]. The previous results with Miller-Spencer treatment of SRC certainly overestimates the quenching due to short-range correlations. Of course, the results obtained with the CCM $\mathrm{SRC}$ are preferable. We note that in the case of Bonn-CD CCM SRC the $0 \nu \beta \beta$-decay NMEs are slightly increased [174. 
The two-nucleon short range correlations were treated also through the UnitaryCorrelation Operator Method (UCOM) [300, 301, 310, which has the advantages of wave-function overlap preservation and a range of successful applications [311]. The drawback of this approach, when applied to the $0 \nu \beta \beta$-decay, is that it violates some general properties of the Fermi and Gamow-Teller matrix elements [174].

Recently, a question of many-body short-range correlations in the evaluation of the $0 \nu \beta \beta$-decay NMEs was addressed within a simple model [312]. The existing calculations include long-range many-body correlations in model dependent (ISM, QRPA, PHFB, EDF, IBM) nuclear wave functions but allow only two particles to be correlated at short distances. There are some indications that it is not sufficient.

iv) The effect of deformation.

The nuclei undergoing double beta decay, which are of experimental interest, are spherical or weakly deformed nuclei with exception of ${ }^{150} \mathrm{Nd}$, which is strongly deformed. It was found in [313] that deformation introduces a mechanism of suppression of the $2 \nu \beta \beta$-decay matrix element which gets stronger when deformations of the initial and final nuclei differ from each other [313, 314]. A similar dependence of the suppression of both $M^{2 \nu}$ and $M_{\nu}^{0 \nu}$ matrix elements on the difference in deformations has been found in the PHFB [315, 316] and the ISM [236]. The NMEs have a well-defined maximum when the deformations of parent and daughter nuclei are similar, and they are quite suppressed when the difference in the deformations is large. The ISM results suggest that a large mismatch of deformation can reduce the matrix elements by factors as large as 23. Within the IBM-2 the effects of the deformation are introduced through the bosonic neutron-proton quadrupole interaction. For weakly deformed nuclei the effect is a reduction by about $20 \%$.

The QRPA calculation of the $0 \nu \beta \beta$-decay NMEs requires a construction of all states of the intermediate nucleus, even if closure approximation is considered. The results were obtained in spherical limit, which is a significant simplication. Recently, the proton-neutron deformed QRPA with a realistic residual NN interaction was developed [184, 317, 318, 319]. This approach was applied in the case of ${ }^{76} \mathrm{Ge},{ }^{150} \mathrm{Nd}$ and ${ }^{160} \mathrm{Gd}$ and lead to the conclusion that the $0 \nu \beta \beta$-decay of ${ }^{150} \mathrm{Nd}$, to be measured soon by the $\mathrm{SNO}+$ collaboration, provides one of the best probes of the Majorana neutrino mass [184, 319].

v) The occupancies of individual orbits.

The occupancies of valence neutron and proton orbits determined experimentally represent important constraints for nuclear models used in the evaluation of the $0 \nu \beta \beta$ decay NME. For the ${ }^{76}$ Ge and ${ }^{76}$ Se they have been extracted by accurate measurements of one nucleon adding and removing transfer reactions by J. Schiffer and collaborators [320, 321]. The main motivation to study these nuclei was the fact that they are the initial and final states of $0 \nu \beta \beta$-decay transitions. These measurements offer a possibility to compare these experimental results with the theoretical occupations and, if necessary, detect which modifications would be required in the mean field or the effective interaction in order to obtain improved agreement with the experiment. 
In a theoretical study 298] measured proton and neutron occupancies were used as a guideline for a modification of the effective mean field energies, which resulted in a better description of these quantities. The calculation of the $0 \nu \beta \beta$-decay NME for ${ }^{76} G e$ performed with an adjusted Woods-Saxon mean field combined with the selfconsistent RQRPA (SRQRPA) method [298], which conserves the mean particle number in correlated ground state, led to a reduction of $M_{\nu}^{0 \nu}$ by $20 \%-30 \%$ when compared to the previous QRPA values.

In the ISM the variation of the nuclear matrix element (NME) for $0 \nu \beta \beta$-decay of ${ }^{76} \mathrm{Ge}$ was studied after the wave functions were constrained to reproduce the experimental occupancies of the two nuclei involved in the transition. It was found 322] that in the ISM description the value of the NME is enhanced about $15 \%$ compared to previous calculations. This diminishes the discrepancies between the ISM and the QRPA approaches.

The role of occupancies of the single-particle orbitals in the standard QRPA calculation of $M_{\nu}^{0 \nu}$ to ground and $0_{1}^{+}$states of final nucleus were studied also in [323, 324]. Unlike the treatment of [298], whereby occupancies in respect to the correlated SRQRPA ground state were considered, the occupancies were evaluated at the level of uncorrelated BCS ground state. The basic features of the ground and excited state decays were found to be quite different.

vi) The axial-vector coupling constant $g_{A}$.

It is well known that the calculated strengths of GamowTeller $\beta$-decay transitions to individual final states are significantly larger than the experimental ones. That effect is known as the axial-vector current matrix elements quenching. To account for this, it is customary to quench the calculated GT matrix elements up to $70 \%$. Formally, this is accomplished by replacing the true value of the coupling constant $g_{A}=1.269$ (the previously $g_{A}=1.254$ was considered) by a quenched value $g_{A}^{\text {eff }}=1.0$. The origin of the quenching is not completely known. This effect is assigned to the $\Delta$-isobar admixture in the nuclear wave function or to the shift of the GT strength to higher excitation energies due to the short-range tensor correlations. It is not clarified yet whether similar phenomenon exists for other multipoles, besides $J=1^{+}$.

Quenching is very important for the double beta decay because $g_{A}^{e f f}$ appears to the fourth power in the decay rate. If it occurs also for the $0 \nu \beta \beta$-decay, it could significantly reduce the $0 \nu \beta \beta$-decay half-life by as much as a factor of $2-3$. The axial-vector coupling constant $g_{A}^{e f f}$ or in other words, the treatment of quenching, is also a source of differences in the calculated $0 \nu \beta \beta$-decay NMEs. $M^{\prime 0 \nu}$ is a function of squared $g_{A}^{e f f}$, which appears by vector and weak-magnetism terms following the definition of Eq. (141).

In [325] three independent lifetime data $(2 \nu \beta \beta$-decay, EC, $\beta$-decay) were accurately reproduced in the QRPA by means of two free parameters $\left(g_{p p}, g_{A}^{e f f}\right)$, resulting in an overconstrained parameter space. The general trend in favor of $g_{A}^{\text {eff }}<1$ was confirmed. This novel possibility to reconcile QRPA results with experimental data, which deserves further discussions and tests, warrants a reconsideration of the quenching problem from a new perspective. 
As it was manifested above nuclear NMEs for $0 \nu \beta \beta$-decay are affected by relatively large theoretical uncertainties. Within the QRPA approach, it was shown that, within a given set of nuclei, the correlations among NME errors are as important as their size 326. This represents a first attempt to quantify the covariance matrix of the NMEs, and to understand its effects in the comparison of current and prospective $0 \nu \beta \beta$-decay results for two or more nuclei. It would be useful if other theoretical groups in the $0 \nu \beta \beta$ field could present "statistical samples" of NME calculations as well, in order to provide independent estimates of (co)variances for their NME estimates. A covariance analysis like the one proposed [326] represents a useful tool to estimate correctly current or prospective sensitivities to effective Majorana neutrino mass $\left\langle m_{\nu}\right\rangle$.

Currently, the uncertainty in calculated $0 \nu \beta \beta$-decay NMEs can be estimated up to factor of 2 or 3 depending on the considered isotope, mostly due to differences between the ISM results and the results of other approaches (QRPA, PHFB, EDF, IBM) and also due to unknown value of $g_{A}^{e f f}$.

\subsection{Anatomy of NMEs}

The anatomy of the $0 \nu \beta \beta$-decay NME was performed in [236, 293. $M_{\nu}^{0 \nu}$ was decomposed on the angular momenta and parities $\mathcal{J}^{\pi}$ of the pairs of neutrons that are transformed into protons with the same $\mathcal{J}^{\pi}$. It was found that the final value of $M_{\nu}^{0 \nu}$ reflects two competing forces: the like particle pairing interaction that leads to the smearing of Fermi levels and the residual neutron-proton interaction that, through ground state correlations, admixes "broken-pair" (higher-seniority) states. The function $C^{0 \nu}(r)$ that describes the dependence of the $M_{\nu}^{0 \nu}$ on internucleon distances r,

$$
M_{\nu}^{0 \nu}=\int_{0}^{\infty} C^{0 \nu}(r) d r
$$

was subject of interest. It was shown that the above competition implies that only internucleon distances $r<2-3 \mathrm{fm}$ contribute to $M_{\nu}^{0 \nu}$ [293]. The maximum value of $C^{0 \nu}(r)$ occurs around $\mathrm{r}=1 \mathrm{fm}$, which means that almost the complete value of $M_{\nu}^{0 \nu}$ comes from contribution of decaying nucleons which are close to each other. This distance correspond to a neutrino momentum of $q \approx 200 \mathrm{MeV}$, twice larger value as was expected before. This finding, which explains a small spread of results for different nuclei, was confirmed also by the ISM [236] and a similar behavior for $C^{0 \nu}(r)$ was obtained also within the PHFB [129]. The QRPA and ISM functions $C^{0 \nu}(r)$ differ only by a scaling factor, which is expected to be related with the ratio of the average number of pairs in both calculations.

The largest component of $M_{\nu}^{0 \nu}$ is the GT part. We have

$$
M_{\nu}^{0 \nu}=M_{G T}^{0 \nu}\left(1+\chi_{F}+\chi_{T}\right),
$$

where $\chi_{F}$ and $\chi_{G T}$ are matrix element ratios that are smaller than unity and, presumably, less dependent on the details of the applied nuclear model. In [327] it was shown that $M_{G T}^{0 \nu}$ is related to the closure $2 \nu \beta \beta$-decay NME $M_{c l}^{2 \nu}$. That relation is 
revealed when these matrix elements are expressed as functions of the relative distance between the pair of neutrons that are transformed into a pair of protons. We have

$$
C_{G T}^{0 \nu}(r)=H_{G T}(r, \bar{E}) C_{c l}^{2 \nu}(r)
$$

where $H(r, \bar{E})$ is the neutrino exchange potential in nucleus and $C_{c l}^{2 \nu}(r)$ is defined as

$$
M_{c l}^{2 \nu}=\int_{0}^{\infty} C_{c l}^{2 \nu}(r) d r .
$$

While the matrix element $M_{c l}^{2 \nu}$ get contributions only from $1^{+}$intermediate states, the function $C_{c l}^{2 \nu}(r)$ gets contributions from all intermediate multipoles.

The Eq. (162) represents the basic relation between the $0 \nu \beta \beta$ - and $2 \nu \beta \beta$-decay modes. An analysis of this relation allowed to explained the contrasting behavior of $M_{G T}^{0 \nu}$ and $M_{c l}^{2 \nu}$ when $\mathrm{A}$ and $\mathrm{Z}$ is changed, namely that $M_{G T}^{0 \nu}$ changes slowly and smoothly unlike $M_{c l}^{2 \nu}$, which has pronounced shell effects [327].

In 328] a connection of the Fermi $0 \nu \beta \beta$-decay NME $M_{F}^{0 \nu}$ with an energy-weighted double Fermi transition matrix element was presented. It is argued that $M_{F}^{0 \nu}$ can be reconstructed, if the isospin-forbidden Fermi transition between the ground state of the final nucleus and the isobaric analog state in the intermediate nucleus can be measured, e.g. by means of (n,p) charge-exchange reactions. By knowing $M_{F}^{0 \nu}$ one can evaluate $M^{0 \nu}$ by assuming an approximate relation $M_{F}^{0 \nu} / M_{G T}^{0 \nu} \approx-2.5$, which follows from the QRPA calculations [174.

\section{Distinguishing the $0 \nu \beta \beta$-decay mechanisms}

Many extensions of the SM generate Majorana neutrino masses and offer a plethora of $0 \nu \beta \beta$-decay mechanisms. Among these we should mention the exchange of heavy neutrinos, the exchange of SUSY superpartners with R-parity violation, leptoquarks, right-handed $\mathrm{W}$ bosons, or Kaluza-Klein excitations, among others, which have been discussed in the previous sections or can be found in the literature [68].

An unambiguous detection of $0 \nu \beta \beta$-decay will prove that the total lepton number is broken in nature and neutrinos are Majorana particles. However, after neutrino oscillations have established that the neutrinos are massive, as we have already mentioned, the observation of $0 \nu \beta \beta$-decay is expected to play a crucial role in determining the neutrino mass scale. This prospect generates the questions: What is the mechanism that triggers the decay? What happens if several mechanisms are active for the decay?

\subsection{Dominance of a single mechanism}

Usually, the $0 \nu \beta \beta$-decay is discussed by assuming that one mechanism at a time dominates. Then the half-life in a given nucleus $i \equiv(A, Z)$ can be written as

$$
\left(T_{1 / 2}^{0 \nu}(i)\right)^{-1}=\left|\eta_{\kappa}\right|^{2}\left|M_{\kappa}^{\prime 0 \nu}(i)\right|^{2} G_{\kappa}^{0 \nu}(i) .
$$


Here, $\eta_{\kappa}, M^{\prime 0 \nu}, G_{\kappa}^{0 \nu}(A, Z)$ are the LNV parameter ( $\kappa$ denotes a given mechanism of the $0 \nu \beta \beta$-decay), associated NME and kinematical factor, respectively. The calculation of $M_{\kappa}^{\prime 0 \nu}=\left(g_{A}^{e f f} / g_{A}\right)^{2} M_{\kappa}^{0 \nu}\left(M_{\kappa}^{0 \nu}\right.$ in some cases depends also on $\left.g_{A}^{\text {eff }}\right)$ allows to deduce constraint on $\eta_{\kappa}$ from the measured lower bound on the $0 \nu \beta \beta$-decay half-life. The definition of ${M^{\prime}{ }_{\kappa} \nu}$ in (164) allows to display the effects of uncertainties in $g_{A}^{\text {eff }}$ and to use the same phase factor $G_{\kappa}^{0 \nu}$ when calculating the $0 \nu \beta \beta$-decay rate [291, 292].

In connection with the neutrino oscillations much attention is attracted to the light neutrino mass mechanism of the $0 \nu \beta \beta$-decay $\left(\eta_{\nu}=\left\langle m_{\nu}\right\rangle / m_{e}\right)$ (see section 4). Small neutrino masses and neutrino mixing are commonly considered as a signature of physics beyond the SM. Several beyond the SM mechanisms of neutrino-mass generation were proposed. The most viable and plausible mechanism is the famous see-saw mechanism which is based on the assumption that the total lepton number $\mathrm{L}$ is violated at a scale much larger than the electroweak scale.

The $0 \nu \beta \beta$-decay is ruled by the light Majorana neutrino-mass mechanism in the case of the standard see-saw mechanism of neutrino-mass generation, which is based on the assumption that the lepton number is violated at a large $\left(10^{15} \mathrm{GeV}\right)$ scale. In [170] it was shown that if $0 \nu \beta \beta$-decay will be observed in future experiments sensitive to the effective Majorana mass in the inverted mass hierarchy region, then a comparison of the derived ranges with measured half-lives will allow us to probe the standard see-saw mechanism (see section 3), assuming that future cosmological data will establish the sum of the neutrino masses to be about $0.2 \mathrm{eV}$.

A primary purpose of type I see-saw, see section 3, which is the simplest extension of the SM, is to account for light neutrino masses in a renormalizable gauge model. Only heavy sterile neutrino states are added to the spectrum of the $S U(3)_{C} \times S U(2)_{L} \times U(1)_{Y}$ theory. These heavy states might lead to measurable effects also for the $0 \nu \beta \beta$-decay. The possible contribution of sterile neutrino dominated Majorana mass eigenstate $\nu_{h}$ with mass $m_{h}$ to the $0 \nu \beta \beta$-decay was examined in [329]. From the most stringent lower bound on the $0 \nu \beta \beta$-decay half-life of ${ }^{76} \mathrm{Ge}$ upper limits on the neutrino mixing matrix element $\left|U_{e h}\right|^{2}$ in wide region of values of $m_{h}$ (below and above TeV scale) were derived. It was assumed that the value of $\left|\left\langle m_{\nu}\right\rangle\right|$ is significantly smaller than the current limit on this quantity $\left(\left|\left\langle m_{\nu}\right\rangle\right| \ll 0.2-0.3 \mathrm{eV}\right)$.

Recently, the $0 \nu \beta \beta$-decay associated with the exchange of virtual sterile neutrinos, that mix with ordinary neutrinos and are heavier than $200 \mathrm{MeV}+$, was revisited [330]. The question of having a dominant heavy sterile neutrino contribution in $0 \nu \beta \beta$-decay was explored in detail. Due to the improved result of the NMEs [331, 191], the bounds on active-sterile mixing coming from $0 \nu \beta \beta$-decay has become one order of magnitude stronger. The possibility that the sterile neutrino contribution become dominant over the light neutrino contribution was addressed for the two flavor and the three flavor scenarios [330]. The dominant sterile neutrino contribution in $0 \nu \beta \beta$ process provide a way to overcome the conflict between cosmology and the claim for evidence of the

+ The case of light sterile neutrinos has already been discussed in section 4 . 
$0 \nu \beta \beta$-decay by Klapdor and collaborators [41, 42].

There is a possibility that the total lepton number is violated at $\mathrm{TeV}$ scale [332, 333, 334, 335, which is accessible at the Large Hadron Collider. The Large Hadron Collider can determine the right-handed neutrino masses and mixings. In [333] it was manifested that the discovery of left-right (LR) symmetry at the Large Hadron Collider would provide a strong motivation for $0 \nu \beta \beta$ searches. By exploiting the LR model with type-II see-saw (see section 3) it was shown that the exchange of heavy neutrinos may dominate the $0 \nu \beta \beta$-decay rate depending on the mass of right-handed charged gauge boson and the mixing of right-handed neutrinos [333] (see Eq. (67)). A complementary study of lepton-flavor violating processes (e.g., $\mu \rightarrow e \gamma$ ), which can provide constraints on masses of right-handed neutrinos and doubly charged scalars is of great importance [333, 336].

The LR symmetric models [17, 18, 337] are popular models of particle physics due to restoration of parity at high energy scale and because they can naturally account for the smallness of neutrino masses. They allow not only the light and heavy neutrino mass mechanisms of the $0 \nu \beta \beta$-decay but also those associated with effective neutrino mass independent parameters $\langle\eta\rangle$ and $\langle\lambda\rangle$, see section 5.2. As it was showed already before there is an exchange of light neutrinos between two $\beta$-decaying nucleons in nucleus in this case.

The three terms $\left\langle m_{\nu}\right\rangle,\langle\eta\rangle$ and $\langle\lambda\rangle$ in the $0 \nu \beta \beta$-decay rate show different characteristics in the angular correlations and energy spectrum [63]. By knowing the single electron energy spectrum and the angular correlation of the two electrons with sufficient accuracy, one could distinguish between decays due to coupling to the left handed and right-handed hadronic currents [63]. This possibility was studied in the context of ${ }^{76} \mathrm{Ge}$ and SuperNEMO (Isotopes under consideration for SuperNEMO are ${ }^{82} \mathrm{Se},{ }^{150} \mathrm{Nd}$ and $\left.{ }^{48} \mathrm{Ca}[180,338]\right)$ detectors. In [339, 340, 341, 342] the expected pulse shapes to be observed for the $0 \nu \beta \beta$-decay events in a big ${ }^{76} \mathrm{Ge}$ detector have been calculated starting from their Monte Carlo calculated time history and spatial energy distribution. The conclusion was that with the spatial resolution of a large size Ge detector for the majority of $0 \nu \beta \beta$ events it is not possible to differentiate between the contributions of $\left\langle m_{\nu}\right\rangle$ and the right-handed weak current parameters $\langle\eta\rangle$ and $\langle\lambda\rangle$. Contrary, the SuperNEMO experiment [180, 338] has a unique potential to measure the decay electron's angular and energy distributions and thus to disentangle these possible mechanisms for $0 \nu \beta \beta$ decay [180, 343]. We note that the planned experiment SuperNEMO, which allows the measurement of $0 \nu \beta \beta$-decay in several isotopes to both the ground and excited states, is able to track the trajectories of the emitted electrons and determine their individual energies. We should mention here that a measurement to both states can also be useful in reducing the background [344]. Other planned experiments that will be able to measure the energy and angular distributions are EXO [177, MOON [345] and COBRA [346].

There is a motivation to consider the $0 \nu \beta \beta$-decay rate in a general framework, parameterizing the new physics contributions in terms of all effective low-energy currents 
allowed by Lorentz-invariance [201, 347], e.g. within the effective field theory 348]. This approach allows to separate the nuclear physics part of the $0 \nu \beta \beta$-decay from the underlying particle physics model, and derive limits on arbitrary lepton number violating theories. A general Lorentz invariant effective Lagragian for leptonic and hadronic charged weak currents was used to perform a comparative analysis of various $0 \nu \beta \beta$-decay long-range mechanisms in [349, 350]. It was shown that by measuring of angular correlations of emitted electrons in the $0 \nu \beta \beta$-decay together with the ability of observing these decays in several nuclei, would help significantly in identifying the dominant mechanism underlying this process.

There is a class of $0 \nu \beta \beta$-decay mechanisms, which one cannot distinguish from each other kinematically. The light $\left(\eta_{\nu}\right)$ and heavy $\left(\eta_{N}^{L}, \eta_{N}^{R}\right)$ Majorana neutrino mass, the trilinear R-parity breaking mechanisms - both the short-range mechanism $\left(\eta_{\lambda^{\prime}}\right)$ with the exchange of heavy superpartners (gluino and squarks and/or neutralinos and selectron) [27, 88, 89, 351] and the long-range mechanism $\left(\eta_{\tilde{q}}\right)$ involving both the exchange of heavy squarks and light neutrino 202] (called squark-neutrino mechanism), constitute such a group. A discussed possibility to distinguish between these mechanisms is a comparison of results for $0 \nu \beta \beta$-decay in two or more isotopes [352, 353, 354].

Under the assumption of a dominance of a single mechanism of the $0 \nu \beta \beta$-decay the LNV parameter $\eta_{\kappa}$ drops out in the ratio of experimentally determined half-lives for two different isotopes. This ratio depend on the mechanism of the $0 \nu \beta \beta$-decay due nuclear matrix elements and kinematical factors, but is free of LNV parameter. Thus it can be compared with the theoretical prediction for different mechanisms. In addition, it is assumed that in ratio of nuclear matrix elements theoretical uncertainties are reduced due to cancellations of systematic effects. Relative deviations of half-life ratios for various new physics contributions, which were normalized to the half-life of ${ }^{76} \mathrm{Ge}$ and compared to the ratio in the light neutrino mass mechanism, were studied in [354]. It was found that the change in ratios of half-lives varies from $60 \%$ for supersymmetric models up to a factor of 520 for extra-dimensional and LR-symmetric mechanisms. It is concluded that complementary measurements in different isotopes would be strongly encouraged 354, 355].

Another possibility to distinguish between the various $0 \nu \beta \beta$-decay mechanisms is a study of the branching ratios of $0 \nu \beta \beta$-decays to excited $0^{+}\left[352,356\right.$, 3nd $2^{+}$[64, 357] states and a comparative study of the $0 \nu \beta \beta$-decay and neutrinoless electron capture with emission of positron $\left(0 \nu E C \beta^{+}\right)$[358]. Unfortunately, the search for the $0 \nu E C \beta^{+}$. decay is complicated due to small rates and the experimental challenge to observe the produced $\mathrm{x}$ rays or Auger electrons, and most double beta experiments of the next generation are not sensitive to electron tracks or transitions to excited states.

\subsection{Nuclear matrix elements of exotic mechanisms}

Recently, the most interest was paid to the calculation of NMEs associated with the light neutrino mass mechanism and ground state to ground state transition. Less progress 
was achieved in the calculation of NMES of exotic $0 \nu \beta \beta$-decay mechanisms.

Experimental studies of transitions to an excited $0_{1}^{+}$and $2_{1}^{+}$final states $*$ allow us to reduce the background by gamma-electron coincidences. Drawbacks are lower Q values and suppressed nuclear matrix elements. The theoretical studies of the corresponding nuclear transitions were performed within the ISM [236], HartreeFockBogoliubov [357] and QRPA [324, 356, 359, 360] approaches. In the ISM the $0 \nu \beta \beta$-decays of ${ }^{48} \mathrm{Ca},{ }^{76} \mathrm{Ge}$, ${ }^{82} \mathrm{Se},{ }^{124} \mathrm{Sn},{ }^{130} \mathrm{Te}$, and ${ }^{136} \mathrm{Xe}$ to $0_{1}^{+}$excited final state were found at least 25 times more suppressed with respect to the ground state to ground state transition in the case of light neutrino mass mechanism. A similar conclusion was found for the $0 \nu \beta \beta$-decays of ${ }^{76} \mathrm{Ge}$, ${ }^{82} \mathrm{Se},{ }^{100} \mathrm{Mo}$ and ${ }^{136} \mathrm{Xe}$ to the excited collective $0^{+}$state suggesting a suppression $10-100$ larger than that of the transition to ground state [324, 356, 359]. In addition to light neutrino mass also right-handed current [359, 360] and R-parity breaking mechanisms [356] were considered.

Quite the opposite is claimed in a different study [360], namely it was found that the transition rate of the $0 \nu \beta \beta$-decay of ${ }^{76} \mathrm{Zr}$ to first excited $0^{+}$state is favored by the enhanced transition matrix elements attributed to the monopole-vibrational structure of this state.

The $0 \nu \beta \beta$-decay of ${ }^{76} \mathrm{Ge}$ and ${ }^{100} \mathrm{Mo}$ to $2_{1}^{+}$final state was investigated for light neutrino mass and right-handed current mechanisms by taking into account recoil corrections to the nuclear currents in [357]. The initial $0^{+}$and final $2_{1}^{+}$nuclear states were described in terms of the Hartree-Fock-Bogoliubov type wave functions, which were obtained by a variation after particle-number and angular-momentum projection [361. By the numerical calculation of relevant NMEs, it was found that the relative sensitivities of $0^{+} \rightarrow 2^{+}$decays to $\left\langle m_{\nu}\right\rangle$ and $\langle\eta\rangle$ are comparable to those of $0^{+} \rightarrow 0^{+}$ decays. At the same time it was noted that the $0^{+} \rightarrow 2_{1}^{+}$decay is relatively more sensitive to $\langle\lambda\rangle$. We should remind the reader that the observation of $0^{+} \rightarrow 2^{+}$transition does not establish the presence of right handed currents. For a more complete analysis one should consider not only the right handed currents, but supersymmetric contribution as well (see the comment at the end of section 6)

The right-handed current mechanisms are associated with many different NMEs. Within the nuclear shell model they were evaluated just for $0 \nu \beta \beta$-decay of ${ }^{48} \mathrm{Ca}$ to the final ground state 73 . The VAMPIR approach was exploited to calculate them in the case of ${ }^{76} \mathrm{Ge}$ [361]. Many calculations of NMEs related to right-handed current mechanisms were performed within the QRPA and for all nuclei of experimental interest [359, 360, 362, 363, 190]. However, they do not include recent improvements concerning the fixing of parameter space of nuclear Hamiltonian [291, 292, 293, 303] and concerning the description of two-nucleon short-range correlations [174].

There is a revived interest to heavy neutrino mass $\left(\eta_{N}^{L, R}\right)$ and R-parity breaking supersymmetric $\left(\eta_{\lambda^{\prime}}, \eta_{\tilde{q}}\right)$ mechanisms of the $0 \nu \beta \beta$-decay. The NMEs governing these

* As we have already mentioned, transitions to non zero angular momentum final states can only occur via the leptonic $j_{L}, j_{R}$ interference term associated with the $\langle\lambda\rangle$ and $\langle\eta\rangle$ parameters. In this section we will refer to it as right handed current contribution. 
Table 4: Nuclear matrix elements $M_{\nu}^{\prime 0 \nu}$ (light Majoran neutrino mass mechanism), $M_{N}^{\prime 0 \nu}$ (heavy Majorana neutrino mass mechanism), $M_{\lambda^{\prime}}^{\prime 0 \nu}$ (trilinear R-parity breaking SUSY mechanism) and $M_{\tilde{q}}^{\prime 0 \nu}$ (squark mixing mechanism) for the $0 \nu \beta \beta$-decays of ${ }^{76} \mathrm{Ge}$, ${ }^{82} \mathrm{Se}$, ${ }^{100} \mathrm{Mo},{ }^{130} \mathrm{Te}$ and ${ }^{136} \mathrm{Xe}$ within the Selfconsistent Renormalized Quasiparticle Random Phase Approximation (SRQRPA). $\mathrm{R}=1.1 \mathrm{fm} A^{1 / 3}$ is assumed.

\begin{tabular}{|c|c|c|c|c|c|c|}
\hline$\overline{\text { Nucleus }}$ & NN pot. & $\overline{g_{A}^{e f f}}$ & $\left|M_{\nu}^{\prime 0 \nu}\right|$ & $\overline{\left|M_{N}^{\prime 0 \nu}\right|}$ & $\left|M_{\lambda^{\prime}}^{\prime 0 \nu}\right|$ & $\left|M_{\tilde{q}}^{\prime 0 \nu}\right|$ \\
\hline \multirow[t]{4}{*}{${ }^{76} \mathrm{Ge}$} & Argonne & 1.25 & 5.44 & 265 & 700 & 718 \\
\hline & & 1.00 & 4.39 & 196 & 461 & 476 \\
\hline & CD-Bonn & 1.25 & 5.82 & 412 & 596 & 728 \\
\hline & & 1.00 & 4.69 & 317 & 393 & 483 \\
\hline \multirow[t]{4}{*}{${ }^{82} \mathrm{Se}$} & Argonne & 1.25 & 5.29 & 263 & 698 & 710 \\
\hline & & 1.00 & 4.18 & 193 & 455 & 465 \\
\hline & CD-Bonn & 1.25 & 5.66 & 408 & 594 & 720 \\
\hline & & 1.00 & 4.48 & 312 & 388 & 472 \\
\hline \multirow[t]{4}{*}{${ }^{100} \mathrm{Mo}$} & Argonne & 1.25 & 4.79 & 260 & 690 & 683 \\
\hline & & 1.00 & 3.91 & 192 & 450 & 449 \\
\hline & CD-Bonn & 1.25 & 5.15 & 404 & 589 & 691 \\
\hline & & 1.00 & 4.20 & 311 & 384 & 455 \\
\hline \multirow[t]{4}{*}{${ }^{130} \mathrm{Te}$} & Argonne & 1.25 & 4.18 & 240 & 626 & 620 \\
\hline & & 1.00 & 3.34 & 177 & 406 & 403 \\
\hline & CD-Bonn & 1.25 & 4.70 & 385 & 540 & 641 \\
\hline & & 1.00 & 3.74 & 294 & 350 & 416 \\
\hline \multirow[t]{4}{*}{${ }^{136} \mathrm{Xe}$} & Argonne & 1.25 & 2.75 & 160 & 428 & 418 \\
\hline & & 1.00 & 2.19 & 117 & 277 & 271 \\
\hline & CD-Bonn & 1.25 & 3.36 & 172 & 460 & 459 \\
\hline & & 1.00 & 2.61 & 125 & 297 & 297 \\
\hline
\end{tabular}

mechanisms were calculated only within the QRPA [28, 101] with exception of the PHFB calculation for the heavy neutrino mass mechanism [364], which, however, neglects the role of induced hadron currents.

Recently, nuclear matrix elements $M_{\nu}^{\prime 0 \nu}$ (light neutrino mass mechanism), $M_{N}^{\prime 0 \nu}$ (heavy neutrino mass mechanism), $M_{\lambda^{\prime}}^{\prime 0 \nu}$ (trilinear R-parity breaking SUSY mechanism) and $M_{\tilde{q}}^{\prime 0 \nu}$ (squark-neutrino mechanism) were calculated for the $0 \nu \beta \beta$-decay of ${ }^{76} \mathrm{Ge},{ }^{82} \mathrm{Se}$, ${ }^{100} \mathrm{Mo},{ }^{130} \mathrm{Te}$ and ${ }^{136} \mathrm{Xe}$ within the SRQRPA [191, 331]. Unlike in previous calculations the particle-particle strength was adjusted to the $2 \nu \beta \beta$-decay half-life and the twonucleon short-range correlations derived from same potential as residual interactions, namely from the CD-Bonn and Argonne potentials [174], were considered. These refinements affect mainly heavy neutrino mass NMEs, which became significantly larger. In the case of NMEs related to LNV parameter $\eta_{S U S Y}$ the finite nucleon size effect was 
Table 5: Upper bounds on the lepton number violating parameters $\left\langle m_{\nu}\right\rangle, \eta_{N}^{L, R}, \eta_{\lambda^{\prime}}$ and $\eta_{\tilde{q}}$ deduced from current lower bounds on the half-life $\left(T_{1 / 2}^{0 \nu \text {-exp }}\right)$ of $0 \nu \beta \beta$-decay for ${ }^{76} \mathrm{Ge}$ [36], ${ }^{82} \mathrm{Se},{ }^{100} \mathrm{Mo}$ [38, ${ }^{130} \mathrm{Te}$ [39] and ${ }^{136} \mathrm{Xe}$ [40]. Limits on $\not R_{p}$ SUSY coupling $\lambda_{111}^{\prime}$ and on the products of the trilinear $\not R_{p}$-couplings $\lambda_{11 k}^{\prime} \lambda_{1 k 1}^{\prime}(\mathrm{k}=1,2,3)$ for $\Lambda_{S U S Y}=100 \mathrm{GeV}$ are given by assuming that the gluino and squark masses and the trilinear soft SUSY breaking parameters are approximately equal to a common SUSY breaking scale $\Lambda_{S U S Y}=100 \mathrm{GeV}$. Nuclear matrix elements calculated within the Selfconsistent Renormalized Quasiparticle Random Phase Approximation (CD-Bonn potential, $g_{A}=1.25$, see Table 4) are considered.

\begin{tabular}{cccccccccc}
\hline \hline nucl. & $\begin{array}{c}T_{1 / 2}^{0 \nu-\exp } \\
{[\text { years] }}\end{array}$ & $\begin{array}{c}\left|\left\langle m_{\nu}\right\rangle\right| \\
{[\mathrm{eV}]}\end{array}$ & $\times 1 \eta_{N}^{L, R} \mid$ & $\left|\eta_{\lambda^{\prime}}\right|$ & $\lambda_{111}^{\prime}$ & $\left|\eta_{\tilde{q}}\right|$ & $\lambda_{111}^{\prime} \lambda_{111}^{\prime}$ & $\lambda_{112}^{\prime} \lambda_{121}^{\prime}$ & $\lambda_{113}^{\prime} \lambda_{131}^{\prime}$ \\
& $\times 10^{9}$ & $\times 10^{4}$ & $\times 10^{9}$ & $\times 10^{6}$ & $\times 10^{7}$ & $\times 10^{8}$ \\
\hline${ }^{76} \mathrm{Ge}$ & $1.910^{25}$ & 0.23 & 6.2 & 4.3 & 1.2 & 3.5 & 6.3 & 3.3 & 1.4 \\
${ }^{82} \mathrm{Se}$ & $3.210^{23}$ & 0.85 & 23. & 16. & 2.3 & 13. & 24. & 12. & 5.1 \\
${ }^{100} \mathrm{Mo}$ & $1.010^{24}$ & 0.41 & 10. & 7.1 & 1.5 & 6.0 & 11. & 5.7 & 2.4 \\
${ }^{130} \mathrm{Te}$ & $3.010^{24}$ & 0.27 & 6.4 & 4.5 & 1.2 & 3.8 & 6.9 & 3.6 & 1.5 \\
${ }^{136} \mathrm{Xe}$ & $5.710^{24}$ & 0.26 & 10. & 3.7 & 1.1 & 3.8 & 6.8 & 3.5 & 1.5 \\
\hline \hline
\end{tabular}

taken into account, which plays an important role in the case of one-pion exchange. For large model space and quenched and unquenched value of weak coupling constant NMEs of these four mechanisms are presented in Table 4. We note that a large model space is important to describe reliably especially tensor matrix element contribution to the full matrix element.

The lepton number violating parameters $\left\langle m_{\nu}\right\rangle, \eta_{N}^{L, R}, \eta_{\lambda^{\prime}}$ and $\eta_{\tilde{q}}$ deduced from current lower bounds on the half-life $\left(T_{1 / 2}^{0 \nu-e x p}\right)$ of $0 \nu \beta \beta$-decay for ${ }^{76} \mathrm{Ge}[36],{ }^{82} \mathrm{Se},{ }^{100} \mathrm{Mo}$ [38], ${ }^{130} \mathrm{Te}$ [39] and ${ }^{136} \mathrm{Xe}$ [40] are shown in Table 5, The SRQRPA NMEs of 4, in particular those evaluated with CD-Bonn potential and $g_{A}=1.25$, were considered. We see that upper limits on $\left|\left\langle m_{\nu}\right\rangle\right|$ and $\left|\eta_{\tilde{q}}\right|$ from CUORICINO $\left({ }^{130} \mathrm{Te}\right)$ [39] and KamLAND-Zen ( $\left.{ }^{136} \mathrm{Xe}\right)$ [40] experiments are already comparable with those from the Heidelberg-Moscow $\left({ }^{76} \mathrm{Ge}\right)$ experiment [36]. The running KamLAND-Zen experiment is even slightly more sensitive to the $0 \nu \beta \beta$-decay signal as already finished Heidelberg-Moscow experiment in the case of the gluino exchange mechanism.

The $\eta_{\lambda^{\prime}}$ and $\eta_{\tilde{q}}$ parameters are related with the with the $\not R_{p}$-coupling $\lambda_{111}^{\prime}$ and products of the trilinear $\not_{p}$-couplings $\lambda_{11 k}^{\prime} \lambda_{1 k 1}^{\prime}(\mathrm{k}=1,2,3)$, respectively. The current limits on them, presented in Table 5, have been derived under the conventional simplifying assumptions. We assumed all the squark masses and the trilinear soft SUSY breaking 
parameters $A_{d}$ to be approximately equal to a common SUSY breaking scale $\Lambda_{S U S Y}$. Thus we approximately have [202]

$$
\lambda_{11 k}^{\prime} \lambda_{1 k 1}^{\prime} \leq \epsilon_{k} \frac{1}{\sqrt{T_{1 / 2}^{0 \nu-e x p} G^{01}}} \frac{1}{\left|M_{\tilde{q}}^{\prime 0 \nu}\right|}\left(\frac{\Lambda_{S U S Y}}{100 \mathrm{GeV}}\right)^{3}
$$

with $\epsilon_{k}=\left(1.8 \times 10^{3} ; 94.2 ; 3.9\right)$ calculated for the current quark masses $m_{d}=9 \mathrm{MeV}$, $m_{s}=175 \mathrm{MeV}$ and $m_{b}=4.2 \mathrm{GeV}$. In the case of gluino and neutralino $\not R_{p}$ SUSY mechanisms of the $0 \nu \beta \beta$-decay we obtain

$$
\begin{aligned}
& \lambda_{111}^{\prime} \leq 1.8 \frac{1}{\sqrt{T_{1 / 2}^{0 \nu-e x p} G^{01}}} \frac{1}{\left|M_{\lambda^{\prime}}^{\prime 0 \nu}\right|}\left(\frac{m_{\tilde{q}}}{100 \mathrm{GeV}}\right)^{2}\left(\frac{m_{\tilde{g}}}{100 \mathrm{GeV}}\right)^{1 / 2} \\
& \lambda_{111}^{\prime} \leq 12.5 \frac{1}{\sqrt{T_{1 / 2}^{0 \nu-e x p} G^{01}}} \frac{1}{\left|M_{\lambda^{\prime}}^{\prime 0 \nu}\right|}\left(\frac{m_{\tilde{e}}}{100 \mathrm{GeV}}\right)^{2}\left(\frac{m_{\chi}}{100 \mathrm{GeV}}\right)^{1 / 2}
\end{aligned}
$$

with $m_{\tilde{q}} \simeq m_{\tilde{g}} \simeq m_{\tilde{e}} \simeq m_{\chi}=\Lambda_{S U S Y} . m_{\tilde{q}}, m_{\tilde{g}}, m_{\tilde{e}}$ and $m_{\chi}$ are masses of squark, gluino, selectron and neutralino, respectively. This approximation is well motivated by the constraints from the flavor changing neutral currents.

It goes without saying that the calculated NMEs of light neutrino mass and exotic mechanisms of the $0 \nu \beta \beta$-decay depend on the assumption about the nuclear model. In order to improve their reliability and reliability of the upper limits on the $0 \nu \beta \beta$-decay lepton number violating parameters, further investigations are necessary.

\subsection{Two or more competing mechanisms}

There is a general consensus that a measurement of the $0 \nu \beta \beta$-decay in one isotope does not allow us to determine the underlying physics mechanism. Complementary measurements in different isotopes is very important especially for the case there are competing mechanisms of the $0 \nu \beta \beta$-decay.

In the case of coexisting mechanisms with identical phase space factors, the Eq. (164) is generalized as

$$
\left(T_{1 / 2}^{0 \nu}(i)\right)^{-1}=G^{0 \nu}(i) \sum_{\kappa}\left|\eta_{\kappa} M_{\kappa}^{0 \nu}(i)\right|^{2}
$$

Here, $g_{A}^{e f f}=g_{A}$ is assumed. The parameters $\eta_{\kappa}$ may take either sign leading to constructive or destructive interference in the decay amplitude, if $\mathrm{CP}$ conservation is assumed. In general case of $\mathrm{CP}$ violation they include complex phases. By exploiting the fact that the associated nuclear matrix elements are target dependent, given definite experimental results on a sufficient number of targets, in principle one can determine or sufficiently constrain all LNV parameters including the light neutrino mass term.

In [331] up to four coexisting mechanisms for the $0 \nu \beta \beta$-decay, mediated by light Majorana neutrino exchange $\left(\eta_{\nu}\right)$, heavy Majorana neutrino exchange $\left(\eta_{N}^{L}\right)$, R-parity breaking supersymmetry $\left(\eta_{\lambda^{\prime}}\right)$ ), and squark-neutrino $\left(\eta_{\tilde{q}}\right)$ were considered. Both, constructive or destructive interference in the decay amplitude and the $0 \nu \beta \beta$-decay in four different candidate nuclei $\left({ }^{76} \mathrm{Ge},{ }^{82} \mathrm{Se},{ }^{100} \mathrm{Mo},{ }^{130} \mathrm{Te}\right)$ with NMEs given in Table 


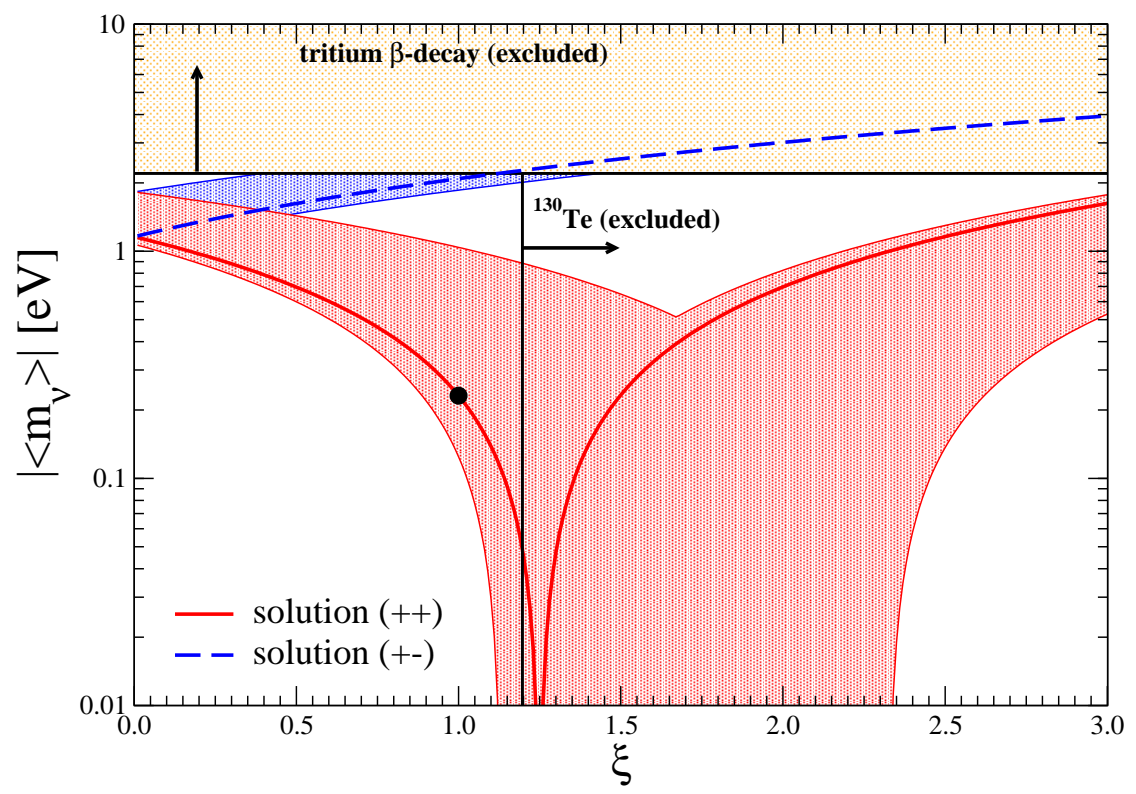

Figure 25: (Color online) The effective Majorana mass of neutrinos in the case of two active mechanisms of the $0 \nu \beta \beta$-decay, namely light and heavy neutrino exchange mechanisms, as function of parameter $\xi$ (see Eq. (169) $)$. Here $T_{1 / 2}^{0 \nu}\left({ }^{76} \mathrm{Ge}\right)=2.23_{-0.31}^{+0.44} \times$ $10^{25}$ y [41, 42] is assumed. Solutions were obtained for equal and opposite signs $(++$, $+-)$ on the left hand side of Eqs. (168). The bold point indicates the value of $\left|\left\langle m_{\nu}\right\rangle\right|$, if the light neutrino exchange is the only active mechanism. The dashed regions showed the uncertainty of the obtained predictions for $\left|\left\langle m_{\nu}\right\rangle\right|$ if a $3 \sigma$ experimental error of the measured half-lives is considered. We also see that a value of $\left|\left\langle m_{\nu}\right\rangle\right|>2.2 \mathrm{eV}$ is excluded due to Mainz tritium $\beta$-decay experiment [166].

4 were assumed. It was found that unfortunately, current NME uncertainties appear to prevent a robust determination of the relative contribution of each mechanism to the decay amplitude, even assuming accurate measurements of $0 \nu \beta \beta$-decay lifetimes.

Another important feature in analysis of two or more competing mechanisms was pointed out in [365]. For example, in the case of two active mechanisms represented by the LNV parameters $\eta_{\nu}=\left\langle m_{\nu}\right\rangle / m_{e}$ and $\eta_{N}^{L}$, assuming the measurement of the $0 \nu \beta \beta$ lifetime of two isotopes $\left(i={ }^{76} \mathrm{Ge},{ }^{130} \mathrm{Te}\right)$ and $\mathrm{CP}$ conservation, one obtains four sets (phases,,,+++--+-- ) of two linear equations:

$$
\frac{ \pm 1}{\sqrt{T_{1 / 2}^{0 \nu}(i) G^{0 \nu}(i)}}=\frac{\left\langle m_{\nu}\right\rangle}{m_{e}} M_{\nu}^{0 \nu}(i)+\eta_{N}^{L} M_{N}^{0 \nu}(i) .
$$

It was found that this improved analysis leads to completely different results compared to those of one mechanism at a time. By making additional assumption that the $0 \nu \beta \beta$ decay of ${ }^{76} \mathrm{Ge}$ was measured with half-life given in [41, 42] the two different solutions 
for $\left|\left\langle m_{\nu}\right\rangle\right|$ are plotted as function of $\xi$, where

$$
\xi=\frac{\left|M_{\nu}^{0 \nu}\left({ }^{130} \mathrm{Te}\right)\right| \sqrt{T_{1 / 2}^{0 \nu}\left({ }^{130} \mathrm{Te}\right) G^{0 \nu}\left({ }^{130} \mathrm{Te}\right)}}{\left|M_{\nu}^{0 \nu}\left({ }^{76} \mathrm{Ge}\right)\right| \sqrt{T_{1 / 2}^{0 \nu}\left({ }^{76} \mathrm{Ge}\right) G^{0 \nu}\left({ }^{76} \mathrm{Ge}\right)}},
$$

in Fig. 25. The parameter $\xi$ represents the unknown half-life of the $0 \nu \beta \beta$-decay of ${ }^{130} \mathrm{Te}$. We note that for $\xi=1$ the solution for active only light neutrino mass mechanism is reproduced and that $\xi=0$ means non-observation of the $0 \nu \beta \beta$-decay for a considered isotope. By glancing the Fig. 25 the obtained results allows to conclude:

i) One of the solutions leads to small values of $\left|\left\langle m_{\nu}\right\rangle\right|$, when all mechanisms add up coherently. This is compatible also with inverted $\left(m_{i}<50 \mathrm{meV}\right)$ or normal $\left(m_{i} \approx\right.$ few $\mathrm{meV}$ ) hierarchy of neutrino masses.

ii) The second solution allows quite large values of $\left|\left\langle m_{\nu}\right\rangle\right|$, even larger than $1 \mathrm{eV}$. It can be excluded by cosmology and tritium $\beta$-decay. However, if the claim for evidence will be ruled out by running (GERDA [43, 366, 367], EXO [177]) and future experiments [31, 368 the values of two solutions will become smaller and perhaps it will not anymore be possible to exclude this solution.

iii) There is possibility that the non-observation of the $0 \nu \beta \beta$-decay for some isotopes could be in agreement with a value of $\left|\left\langle m_{\nu}\right\rangle\right|$ in the sub-eV region.

iv) The obtained results are sensitive to the accuracy of measured half-lives and to uncertainties in calculated nuclear matrix elements.

Other possibilities of getting information about the different LNV parameters in the case of competing $0 \nu \beta \beta$-decay mechanisms were discussed in [191]. First, two competitive mechanisms, namely light left handed Majorana neutrino exchange and heavy right-handed Majorana neutrino exchange, were considered. As the interference term is negligible the $0 \nu \beta \beta$-decay half-life for a given isotope is written as

$$
\left(T_{1 / 2}^{0 \nu}(i) G^{0 \nu}(i)\right)^{-1}=\left|\eta_{\nu} M_{\nu}^{0 \nu}(i)\right|^{2}+\left|\eta_{N}^{R} M_{N}^{0 \nu}(i)\right|^{2},
$$

where the index $\mathrm{i}$ denotes the isotope. As we have mentioned in subsection 5.2 the interference between the left and right handed currents is small. Given a pair of nuclei, solutions for $\left|\eta_{\nu}\right|^{2}$ and $\left|\eta_{N}^{R}\right|^{2}$ can be found by solving a system of two linear equations. From the "positivity" conditions $\left(\left|\eta_{\nu}\right|^{2}>0\right.$ and $\left.\left|\eta_{N}^{R}\right|^{2}>0\right)$ it follows that the ratio of half-lives is within the range [191]

$$
\frac{G^{0 \nu}(i)\left|M_{N}^{0 \nu}(i)\right|^{2}}{G^{0 \nu}(j)\left|M_{N}^{0 \nu}(j)\right|^{2}} \leq \frac{T_{1 / 2}^{0 \nu}(j)}{T_{1 / 2}^{0 \nu}(i)} \leq \frac{G^{0 \nu}(i)\left|M_{\nu}^{0 \nu}(i)\right|^{2}}{G^{0 \nu}(j)\left|M_{\nu}^{0 \nu}(j)\right|^{2}} .
$$

Surprisingly, the physical solutions are possible only if the ratio of the half-lives, in particular of three considered isotopes ${ }^{76} \mathrm{Ge},{ }^{100} \mathrm{Mo}$ and ${ }^{130} \mathrm{Te}$, takes values in very narrow intervals [191].

The $0 \nu \beta \beta$-decay can be triggered also by two competitive mechanisms whose interference contribution to the decay rates is non-negligible. As an example the light Majorana neutrino mass and gluino exchange mechanisms were considered in [191]. We have

$$
\left(T_{1 / 2}^{0 \nu}(i) G^{0 \nu}(i)\right)^{-1}=\left|\eta_{\nu} M_{\nu}^{0 \nu}(i)\right|^{2}+\left|\eta_{\lambda^{\prime}} M_{\lambda^{\prime}}^{0 \nu}(i)\right|^{2}
$$




$$
+2 \cos \alpha\left|\eta_{\nu}\right|\left|\eta_{\lambda^{\prime}}\right|\left|M_{\nu}^{0 \nu}(i)\right|\left|M_{\lambda^{\prime}}^{0 \nu}(i)\right|
$$

Here, $\alpha$ is the relative phase of $\eta_{\nu}$ and $\eta_{\lambda^{\prime}}$. From (172) it is possible to extract the values of $\left|\eta_{\nu}\right|^{2}$ and $\left|\eta_{\lambda^{\prime}}\right|^{2}$ and $\cos \alpha$ setting up a system of three equations with these three unknowns using as input the data on the half-lives of three different nuclei. Results of Ref. [191] show that by using of prospective upper bounds on the absolute scale of neutrino masses stringent constraints on some of new physics mechanisms, which interfere destructively with light neutrino mass mechanism, can be found or even these scenarios can be excluded.

\section{Resonant neutrinoless double electron capture}

As it has already been mentioned in section 2, the resonant $0 \nu$ ECEC, was considered by Winter [50] already in 1955 as a process that would demonstrate the Majorana nature of neutrinos and the violation of the total lepton number. The two asterisks denote the possibility of leaving the system in an excited nuclear and/or atomic state. The energy excess given by the Q-value of the initial atom is carried away by emission of $\mathrm{x}$ rays (or Auger electrons) as the daughter atom has two electron holes and by emission of a single or few photons due to de-excitation of final nucleus.

The possibility of a resonant enhancement of the $0 \nu \mathrm{ECEC}$ in case of a mass degeneracy between the initial and final atoms was pointed out by Bernabéu, De Rujula, and Jarlskog as well as by Vergados about 30 years ago [51, 52]. The half-life of the process was estimated by considering non-relativistic atomic wave functions at nuclear origin, simplified evaluation of corresponding NME and assuming that the degeneracy parameter $\Delta=M_{A, Z}-M_{A, Z-2}^{* *}$, being the difference of masses of the initial and final

excited atoms with masses $M_{A, Z}$ and $M_{A, Z-2}^{* *}$, varies from zero to $10 \mathrm{keV}$ (representing the accuracy of atomic mass measurement at that time). The range of $\Delta$ induced uncertainty of about 5 orders in magnitude in calculated $0 \nu E C E C$ half-life. A list of promising isotopes based on the degeneracy requirement associated with arbitrary nuclear excitation was presented. The ${ }^{112} S n \rightarrow{ }^{112} C d$ resonant $0 \nu$ ECEC transition was identified as a good case.

In 2004 Sujkowski and Wycech [69] and Lukaszuk et al. [369] analyzed the resonant $0 \nu$ ECEC process for nuclear $0^{+} \rightarrow 0^{+}$transitions accompanied by emission of a single photon. By assuming $\left|\left\langle m_{\nu}\right\rangle\right|=1 \mathrm{eV}$ and $1 \sigma$ error in the atomic mass determination the resonant $0 \nu$ ECEC rates of six selected isotopes were calculated by considering the perturbation theory approach. The lowest $0 \nu$ ECEC half-life was found for ${ }^{152} \mathrm{Gd}$.

The main limitation in identifying promising isotopes for experimental search of $0 \nu$ ECEC has been poor experimental accuracy of measurement of $Q$-values which until recently were known with uncertainties of $1-10 \mathrm{keV}$ only [370]. The resonance enhancement can increase the probability of capture by many orders of magnitude. Therefore, accurate mass difference measurements are of great importance in order to narrow down the possibilities. Progress in precision measurement of atomic masses with Penning traps [56, 371, 372] has revived the interest in the old idea on the resonance 
$0 \nu$ ECEC capture. Recently, the accuracy of $Q$-values at around $100 \mathrm{eV}$ was achieved [55, 373, 374, 375, 376, 377, 378, 379, 380, 381, 382, 383], which has already allowed to exclude some of isotopes from the list of the most promising candidates (e.g., ${ }^{112} \mathrm{Sn}$ and ${ }^{164} \mathrm{Er}$ ) for searching the $0 \nu \mathrm{ECEC}$.

Recently, a significant progress has been achieved also in theoretical description of the resonant $0 \nu$ ECEC [54, 70, 384]. A new theoretical framework for the calculation of resonant $0 \nu \mathrm{ECEC}$ transitions, namely the oscillation of stable and quasi-stationary atoms due to weak interaction with violation of the total lepton number and parity, was proposed in [54, 70]. The $0 \nu$ ECEC transition rate near the resonance is of Breit-Wigner form,

$$
\Gamma_{a b}^{0 \nu \operatorname{ECEC}}\left(J^{\pi}\right)=\frac{\left|V_{a b}\left(J^{\pi}\right)\right|^{2}}{\Delta^{2}+\frac{1}{4} \Gamma_{a b}^{2}} \Gamma_{a b},
$$

where $J^{\pi}$ denotes angular momentum and parity of final nucleus. The degeneracy parameter can be expressed as $\Delta=Q-B_{a b}-E_{\gamma}$. $Q$ stands for a difference between the initial and final atomic masses in ground states and $E_{\gamma}$ is an excitation energy of the daughter nucleus. $B_{a b}=E_{a}+E_{b}+E_{C}$ is the energy of two electron holes, whose quantum numbers $(n, j, l)$ are denoted by indices $a$ and $b$ and $E_{C}$ is the interaction energy of the two holes. The binding energies of single electron holes $E_{a}$ are known with accuracy with few eV [385]. The width of the excited final atom with the electron holes is given by

$$
\Gamma_{a b}=\Gamma_{a}+\Gamma_{b}+\Gamma^{*} .
$$

Here, $\Gamma_{a, b}$ is one-hole atomic width and $\Gamma^{*}$ is the de-excitation width of daughter nucleus, which can be neglected. Numerical values of $\Gamma_{a b}$ are about up to few tens eV [386].

For light neutrino mass mechanism and favorable cases of a capture of $s_{1 / 2}$ and $p_{1 / 2}$ electrons the explicit form of lepton number violating amplitude associated with nuclear transitions $0^{+} \rightarrow J^{\pi}=0^{ \pm 1}, 1^{ \pm 1}$ is given in [54]. By factorizing the electron shell structure and nuclear matrix element one get

$$
V_{a b}\left(J^{\pi}\right)=\frac{1}{4 \pi} G_{\beta}^{2} m_{e} \eta_{\nu} \frac{g_{A}^{2}}{R}<F_{a b}>M^{0 \nu E C E C}\left(J^{\pi}\right) .
$$

Here, $\left\langle F_{a b}\right\rangle$ is a combination of averaged upper and lower bispinor components of the atomic electron wave functions [54] and $M^{0 \nu E C E C}\left(J^{\pi}\right)$ is the nuclear matrix element. We note that by neglecting the lower bispinor components $M^{0 \nu E C E C}\left(0^{+}\right)$takes the form of the $0 \nu \beta \beta$-decay NME for ground state to ground state transition after replacing isospin operators $\tau^{-}$by $\tau^{+}$.

There is a straightforward generalization of the LNV potential $V_{a b}\left(0^{+}\right)$in (175) for the heavy neutrino exchange, the trilinear R-parity breaking with gluino and neutralino exchange and squark-neutrino mechanisms. It is achieved by replacements $\eta_{\nu}=\left\langle m_{\nu}\right\rangle / m_{e}$ with $\eta_{\kappa}\left(\kappa=N, \lambda^{\prime}, \tilde{q}\right)$ and $M_{\nu}^{0 \nu E C E C}\left(0^{+}\right)$with $M_{\kappa}^{0 \nu E C E C}\left(0^{+}\right)$. The $0 \nu$ ECEC leading to final states different than $0^{+}$, possible only in the presence weak right-handed currents due to the leptonic of $j_{L}-j_{R}$ interference, has been discussed in 384 . 
New important theoretical findings with respect of the $0 \nu$ ECEC were achieved in [54]. They are as follows: i) Effects associated with the relativistic structure of the electron shells reduce the $0 \nu$ ECEC half-lives by almost one order of magnitude. ii) The capture of electrons from the $n p_{1 / 2}$ states is only moderately suppressed in comparison with the capture from the $n s_{1 / 2}$ states unlike in the non-relativistic theory. iii) For light neutrino mass mechanism selection rules appear to require that nuclear transitions with a change in the nuclear spin $J \geq 2$ are strongly suppressed. We note that, if right-handed currents are considered, selection rules are modified allowing also $J \neq 0^{+}$ [384]. iv) New transitions due to the violation of parity in the $0 \nu$ ECEC process were proposed. For example, nuclear transitions $0^{+} \rightarrow 0^{ \pm}, 1^{ \pm}$are compatible with a mixed capture of s- and p-wave electrons. v) The interaction energy of the two holes $E_{C}$ has to be taken into account by evaluating a mass degeneracy of initial and final atoms. vi) Based on the most recent atomic and nuclear data and by assuming $M^{0 \nu E C E C}\left(J^{\pi}\right)=6$ the $0 \nu$ ECEC half-lives were evaluated and the complete list of the most perspective isotopes for further experimental study was provided. Some isotopes such as ${ }^{156}$ Dy have several closely-lying resonance levels. A more accurate measurement of Q-value of ${ }^{156} \mathrm{Dy}$ by Heidelberg group confirmed the existence of multiple-resonance phenomenon for this isotope [380]. vii) In the unitary limit some $0 \nu$ ECEC half-lives were predicted to be significantly below the $0 \nu \beta \beta$-decay half-lives for the same value of $\left\langle m_{\nu}\right\rangle$. A probability of finding resonant transition with low $0 \nu$ ECEC half-life was evaluated. vii) The process of the resonant neutrinoless double electron production $(0 \nu \mathrm{EPEP})$, i.e. neutrinoless double beta decay to two bound electrons, namely

$$
(A, Z) \rightarrow(A, Z+2)^{* *}+e_{b}^{-}+e_{b}^{-},
$$

was proposed and analyzed. This process was found to be unlikely as it requires that a Q-value is extremely fine tuned to a nuclear excitation. The two electrons must be placed into any of the upper most non-occupied electron shells of the final atom leaving only restricted possibility to match to a resonance condition.

A detailed calculation of the $0 \nu$ ECEC of ${ }^{152} \mathrm{Gd},{ }^{164} \mathrm{Er}$ and ${ }^{180} \mathrm{~W}$ associated with the ground-state to ground-state nuclear transitions was performed in [55, 387, 388]. Improved measurements of Q-value for these transitions with accuracy of about $100 \mathrm{eV}$ [381, 383, 382, 387] were considered. The nuclear matrix elements of ${ }^{152} \mathrm{Gd} \rightarrow{ }^{152} \mathrm{Sm}$, ${ }^{164} \mathrm{Er} \rightarrow{ }^{164} \mathrm{Dy}$ and ${ }^{180} \mathrm{~W} \rightarrow{ }^{180} \mathrm{Hf}$ transitions were calculated within spherical and deformed QRPA [387, 388]. The obtained results excludes ${ }^{164} \mathrm{Er}$ and ${ }^{180} \mathrm{~W}$ from the list of prospective candidates to search for the $0 \nu$ ECEC. The $0 \nu$ ECEC half-life of ${ }^{152} \mathrm{Gd}$ is $2-3$ orders of magnitude longer than the half-life of $0 \nu \beta \beta$ decay of ${ }^{76} \mathrm{Ge}$ corresponding to the same value of $\left\langle m_{\nu}\right\rangle$ and is the smallest known half-life among known $0 \nu$ ECEC transitions at present.

The transition of ${ }^{106} \mathrm{Cd}$ to an excited state of ${ }^{106} \mathrm{Pd}$ with the nuclear excitation energy of $2717.59 \mathrm{keV}$ was calculated in Ref. [389] by making assumption that this is the $0^{+}$state. However, it was noted in [54] that, as long as this level $\gamma$-decays by $100 \%$ into the $3^{+}$state at $1557.68 \mathrm{keV}$, this possibility is excluded. 
Table 6: A comparison of the neutrinoless double beta decay and the resonant neutrinoless double electron capture for light neutrino mass mechanism. The lepton number violating amplitude $V_{a b}\left(J^{\pi}\right)$ is given in Eq. (175)).

\begin{tabular}{|c|c|c|}
\hline & $0 \nu \beta \beta$-decay & $0 \nu E C E C$ \\
\hline definition & $(A, Z) \rightarrow(A, Z+2)+e^{-}+e^{-}$ & $(A, Z)+e_{b}^{-}+e_{b}^{-} \rightarrow(A, Z-2)^{* *}$ \\
\hline formalism & perturbation field theory & oscillation of atoms [70, 54 \\
\hline half-life & $\frac{1}{T_{1 / 2}^{0 \nu}}=\left|\frac{\left\langle m_{\nu}\right\rangle}{m_{e}}\right|^{2} G^{0 \nu}\left|M^{0 \nu}\left(J^{\pi}\right)\right|^{2}$ & $\frac{\ln 2}{T_{1 / 2}^{0 \nu \mathrm{ECEC}}}=\frac{\left|V_{a b}\left(J^{\pi}\right)\right|^{2}}{\left(M_{A, Z}-M_{A, Z-2}^{* *}\right)^{2}+\frac{1}{4} \Gamma_{a b}^{2}} \Gamma_{a b}$ \\
\hline nucl. trans. & $0^{+} \rightarrow 0^{+}, 2^{+}$ & $0^{+} \rightarrow 0^{+}, 0^{-}, 1^{+}, 1^{-}$ \\
\hline \multirow[t]{2}{*}{ fav. at. syst. } & large Q-value (3-4 MeV) & mass difference \\
\hline & & of few tens of eV \\
\hline \multirow{3}{*}{ uncert. in $T_{1 / 2}^{0 \nu}$} & $\begin{array}{l}{ }^{48} \mathrm{Ca},{ }^{76} \mathrm{Ge},{ }^{76} \mathrm{Se},{ }^{100} \mathrm{Mo} \\
{ }^{116} \mathrm{Cd},{ }^{130} \mathrm{Te},{ }^{136} \mathrm{Xe},{ }^{150} \mathrm{Nd}\end{array}$ & $\begin{array}{l}\text { unknown yet }\left({ }^{106} \mathrm{Cd},{ }^{124} \mathrm{Xe} \text {, }\right. \\
\left.{ }^{152} \mathrm{Gd},{ }^{156} \mathrm{Dy},{ }^{168} \mathrm{Yb}, \ldots\left[{ }_{54}\right]\right)\end{array}$ \\
\hline & factor $\sim 4-9$ due & many orders in magn. \\
\hline & to calc. of NME & $\begin{array}{l}\text { up to measured mass diff. } \\
\text { and due to NMEs }\end{array}$ \\
\hline \multirow[t]{2}{*}{ exp. sign. } & peak at end of sum & $\mathrm{x}$ rays or Auger el. \\
\hline & of two el. energy spectra & plus nucl. de-excitation \\
\hline$T_{1 / 2}^{0 \nu-e x p}$ & $>10^{24}-10^{25} \mathrm{y}$ & $>10^{19}-10^{20} \mathrm{y}$ \\
\hline \multirow[t]{3}{*}{ exp. act. } & const. of (0.1-1 ton) exp. & small exper. yet \\
\hline & with sensitivity to inverted & \\
\hline & hierarchy of neutrino masses & \\
\hline \multirow[t]{2}{*}{ background } & $2 \nu \beta \beta$-decay & $2 \nu E C E C$ is strongly \\
\hline & upon resolution of exp. & suppressed \\
\hline
\end{tabular}

There is also an increased experimental activity in the field of the resonant $0 \nu \mathrm{ECEC}$ [71, 390, 391, 392, 393, 394, 395]. The resonant 0 $\nu$ ECEC has some important advantages with respect to experimental signatures and background conditions. The de-excitation of the final excited nucleus proceeds in most cases through a cascade of easy to detect rays. A two- or even higher-fold coincidence setup can cut down any background rate right from the beginning, thereby requiring significantly less active or passive shielding [54]. A clear detection of these $\gamma$ rays would already signal the resonant $0 \nu$ ECEC without any doubt, as there are no background processes feeding those particular nuclear levels. It is worth noting that lepton number conserving ECEC with emission of two neutrinos,

$$
(A, Z)+e_{b}^{-}+e_{b}^{-} \rightarrow(A, Z-2)^{* *}+\nu_{e}+\nu_{e},
$$

is strongly suppressed due to almost vanishing phase space [51, 52, 54]. The ground state to ground state resonant $0 \nu$ ECEC transitions can be detected by monitoring the $\mathrm{x}$ rays or Auger electrons emitted from excited electron shell of the atom. This can be achieved, e.g., by calorimetric measurements. 
Till now, the most stringent limit on the resonant $0 \nu$ ECEC were established for ${ }^{74} \mathrm{Se}$ [394], ${ }^{106} \mathrm{Cd}$ [393] and ${ }^{112} \mathrm{Sn}$ [391]. The ground state of ${ }^{74} \mathrm{Se}$ is almost degenerate to the second excited state at $1204 \mathrm{keV}$ in the daughter nucleus ${ }^{74} \mathrm{Ge}$, which is a $2^{+}$state [396]. The $2 \gamma$-ray cascade has been searched for by using the low-radioactivity detector setup at the Comenius University in Bratislava and $3 \mathrm{~kg}$ of natural selenium. A lower limit for the half-life of $T_{1 / 2}^{0 \nu E C E C} \geq 4.3 \times 10^{19} \mathrm{y}$ was determined [394], which is slightly larger than the value reported in [390]. The resonant $0 \nu$ ECEC transition to the $0_{3}^{+}$ excited state in ${ }^{112} \mathrm{Cd}(1871.0 \mathrm{keV})$ has been investigated in an experiment performed with natural tin in the Modane Underground Laboratory. A lower bound on half-life of $0.92 \times 10^{20} \mathrm{y}$ was established. It is worth noticing that a new mass measurement 376 ] has excluded a complete mass degeneracy for a ${ }^{112} \mathrm{Sn}$ decay and has therefore disfavored significant resonant enhancement of the $0 \nu \mathrm{ECEC}$ mode for this transition. Within the TGV experiment in Modane [393] an interest has also arisen in the 0 $\nu$ ECEC resonant decay mode of ${ }^{106} \mathrm{Cd}$ (KL-capture) to the excited $2741 \mathrm{keV}$ state of ${ }^{106} \mathrm{Pd}$. The spin value of this final state was unknown and it was assumed to be $J=(1,2)^{+}$. After measurements had begun a new value for the spin of the $2741 \mathrm{keV}$ level in ${ }^{106} \mathrm{Pd}$ of $J=4^{+}$was adopted, but, following recent theoretical analysis [54], this channel is now disfavored. Nevertheless the most stringent limit on the $0 \nu$ ECEC half-life of of $1.1 \times 10^{20}$ y was reported [393].

A comparison of the $0 \nu \mathrm{ECEC}$ with the $0 \nu \beta \beta$-decay is presented in Table 6. It is maintained that these two lepton number violating processes are quite different and at different levels of both theoretical and experimental investigation. Precise measurements of Q-values between the initial and final atomic states, additional spectroscopic information on the excited nuclear states (energy, spin and parity) and reliable calculation of corresponding NMEs are highly required to improve predictions of half-lives of the resonant $0 \nu$ ECEC. It is expected that the accuracy of $10 \mathrm{eV}$ in the measurement of atomic masses will be achievable in the near future. The electron binding energy depends on the local physical and chemical environment. An interesting question is whether it possible and, if so, how to manage the atomic structure in such a way as to implement the degeneracy of the atoms and create conditions for the resonant enhancement, as discussed in a recent work [54].

\section{Concluding remarks}

In this review we discussed in some detail the lepton number violating neutrinoless double beta decay and other similar transitions, involving various nuclear isotopes for which ordinary beta decay and e-capture are forbidden or highly suppressed. Both theoretical and experimental aspects were considered.

We have seen that this is a process with long and interesting history with important implications for physics and cosmology, but its observation is still elusive. It is an exotic process, which requires physics beyond the SM. At present a complete theory is missing and, thus, to motivate and guide the experiments we examined a number of reasonable 
viable models, beyond the SM, in particular in connection with the neutrino mass matrix and mixing (see sections 3 and 3.4). Such models predict that lepton number violation, and consequently neutrinoless double beta decay, must occur at some level, implying that the neutrinos are Majorana particles. These models, however, cannot provide a precise determination of the parameters involved, such as the absolute scale of the neutrino mass. So they must be extracted from the experiments, if and when reliable accurate results become available (see section 4 for the neutrino mass). The observed values may, then, be used to differentiate between such models and, hopefully, lead to the ultimate theory.

In order to achieve this goal first such processes must be definitely observed. Then the obtained results must be analyzed by considering the various mechanisms implied by the above models, see sections 5 and 6 for mechanisms involving intermediate neutrinos and other particles respectively. This, however, can only be done, if the corresponding nuclear matrix elements are evaluated with high precision, accuracy and reliability. We have seen that this is a formidable task, since the nuclei that can undergo double beta decay have rather complicated structure.

The evaluation of the nuclear matrix elements involves two steps. In the first step the effective transition operators for each mechanism (see section 9). Special attention must be paid to the proper treatment of these operators at short distances (short range correlations, nucleon current corrections, inclusion of hadrons other than nucleons etc). The second step consists of selecting the proper nuclear model for constructing the wave functions involved in the evaluation of the nuclear matrix elements. Practically all models available in the nuclear theory artillery have been employed. The most prominent are the large basis shell model, the various refinements of the quasi particle random phase approximation (QRPA) and the interacting boson model (IBM). The essential features of these models and the numerical values of the obtained nuclear matrix elements have been summarized in section 10. We have seen that great progress has been made in this direction in recent years and it is encouraging that the nuclear ME obtained with these vastly different nuclear models tend to converge.

We have discussed in section 8 the ongoing, planned and future experiments. We have witnessed great progress in tackling the various background problems, improving the energy resolution and preparing large masses of the needed isotopes. It is, thus, expected that half lives of the order of $10^{26} \mathrm{y}$ can be achieved and, consequently, a sensitivity of a few tens of meV for the average neutrino mass can be reached. This may be sufficient to differentiate between the normal and inverted hierarchy scenarios (see section 4). Furthermore we have seen that various nuclear charge changing nuclear reactions can be employed in an effort to experimentally extract useful information or provide checks for the nuclear matrix elements.

It is clear that the observation of neutrinoless double beta decay will be a great triumph for physics and experimental physics in particular. It will demonstrate that the neutrinos are Majorana particles and there exist lepton number violating interactions in the universe. This, however, will not be the end of the story. The data should be 
analyzed in such a way to determine the mechanism responsible for this process and, in particular, to extract the most important parameter, which is scale of the neutrino mass. Great progress has been in this direction has recently been made as briefly exposed in section 11. In order to unambiguously accomplish this goal, however, the accuracy of the nuclear matrix elements must be further improved.

Finally recent developments, towards the accurate determination of atomic masses as well as the evaluation of the inner shell atomic wave functions and energies, have stimulated interest in experiments involving the resonant neutrinoless double e-capture, see section 12. This new process, if observed, especially in case it leads to negative parity final nuclear states, will greatly facilitate the analysis of determining the dominant mechanism involved in neutrinoless double beta decay.

It is clear that theoretical attempts in the determination of the nuclear matrix elements and experimental efforts towards achieving the observation of neutrinoless double beta decays, involving as many as possible nuclear isotopes and utilizing all available techniques, should be encouraged and supported.

\section{Aknowledgements}

The work of one of the authors (JDV) was supported in part by UNILHC PITN-GA2009-237920 and the DIBOSON Thalis project. F.S acknowledges the support by the VEGA Grant agency under the contract No. 1/0876/12. The authors express their sincere thanks to Rastislav Dvornicky and Rastislav Hodak for the preparation of some of the figures.

\section{Bibliography}

[1] M. Goeppert-Mayer. Phys. Rev., 48:512, 1035.

[2] E. Majorana. Nuovo Cim., 14:171, 1937.

[3] G. Racah. Nuovo Cim., 14:322, 1937.

[4] W. Furry. Phys. Rev., 56:1184, 1939.

[5] H. Primakoff. Phys. Rev., 85:888, 1952.

[6] A. Barabash. Phys. Atom. Nucl., 74:603, 2011.

[7] Jr. R. Davis. Phys. Rev., 97:766, 1955.

[8] E. Fireman. Phys. Rev., 75:323, 1949.

[9] E. Fireman. phys. Rev., 86:451, 1952.

[10] M. G. Ingram and J. H. Reynolds. Phys. Rev., 78:822, 1950.

[11] T. Kirsten, W. Gentner, and O.A. Schaeffer. Z. Physik, 202:273, 1967.

[12] T. Kirsten, W. Gentner, and O. Miller. Z. Naturf. A, 22:1783, 1967.

[13] N. Takaota and K. Ogata. Z. Naturf. A, 21:84, 1966.

[14] B. Srinvasan, O.K. E.C. Alexander, and Manuel. J. Inorg. Nucl. Chem., 34:2381, 1972.

[15] M. Goldhaber, L. Grodzins, and A.W. Sunyar. Phys. Rev., 109:1015, 1958.

[16] M. Doi, T. Kotani, N. Nishiura, K. Okuda, and E. Takasugi. Phys. Lett B., 103:219, 1981.

[17] J. C. Pati and A. Salam. Phys. Rev. D, 10:275, 1974.

[18] R. N. Mohapatra and J. C. Pati. Phys. Rev. D, 11:2558, 1975.

[19] R. N. Mohapatra and G. Senjanović. Phys. Rev. D, 12:1502, 1975.

[20] H. Fritzsch and R. Minkowski. Phys. Rep., 73:67, 1981. 
[21] R. Kuchimanchi and R. N. Mohapatra. Phys. Rev. D, 48:4352, 1993.

[22] A. Rasin C. S. Aulakh, A. Melfo and G. Senjanovic. Phys. Rev. D, 58:115007, 1998.

[23] B. Dutta and R. N. Mohapatra. Phys. Rev. D, 59:015018, 1999.

[24] J. Schechter and J. W. F. Valle. Phys. Rev. D, 25:2951, 1982.

[25] H.V. Klapdor-Kleingrothaus M. Hirsch and S. Kovalenko. Phys. Lett. B, 372:181, 1996.

[26] A. Faessler, S. Kovalenko, F. Šimkovic, and J. Schwieger. Phys. Rev. Lett., 78:183, 1997.

[27] A. Faessler, S. Kovalenko, and F. Šmkovic. Phys. Rev. D, 58:055004, 1998.

[28] A. Wodecki, W. A. Kamiński, and F. Šimkovic. Phys. Rev. D, 60:115007, 1999.

[29] V. I. Tretyak and Yu. G. Zdesenko. At. Dat. Nucl. Dat. Tabl., 80:83, 2002.

[30] H. Ejiri. J. Phys. Soc. Jap., 74:2101, 2005.

[31] F.T. Avignone, S.R. Elliott, and J.Engel. Rev. Mod. Phys., 80:481, 2008.

[32] S. R. Elliott, A. A. Hahn A A, and M. Moe. Phys. Rev. Lett., 59:2020, 1987.

[33] Y. Fukuda et al, The Super-Kamiokande Collaboration, Phys. Rev. Lett. 86, (2001) 5651; ibid 81 (1998) 1562 \& 1158; ibid 82 (1999) 1810 ;ibid 85 (2000) 3999.

[34] Q.R. Ahmad et al, The SNO Collaboration, Phys. Rev. Lett. 89 (2002) 011302; ibid 89 (2002) 011301; ibid 87 (2001) 071301;

K. Lande et al, Homestake Collaboration, Astrophys, J 496, (1998) 505;

W. Hampel et al, The Gallex Collaboration, Phys. Lett. B 447, (1999) 127;

J.N. Abdurashitov al, Sage Collaboration, Phys. Rev. C 80 (1999) 056801;

G.L Fogli et al, Phys. Rev. D 66 (2002) 053010.

[35] K. Eguchi et al. (The KamLAND Collaboration). Phys. Rev. Lett., 90:021802, 2003.

[36] L. Baudis et al. Phys. Rev. Lett., 83:41, 1999.

[37] R. Arnold and others (NEMO3 Collaboration). Phys. Rev. Lett., 95:182302, 2005.

[38] V.I. Tretyak and The NEMOIII collaboration. AIP Conf. Proc., 1417:125, 2011.

[39] C. Arnaboldi et al. (CUORE Collaboration). Phys. Lett. B, 584:260, 2004.

[40] A. Gando et al. Phys. Rev. C, 85:045504, 2012.

[41] H.L. Harney H. V. Klapdor-Kleingrothaus, A. Dietz and I. V. Krivosheina. Mod. Phys. lett. A, $16: 2409,2001$.

[42] H. V. Klapdor-Kleingrothaus and I.V. Krivosheina. Mod. Phys. Lett. A, 21:1547, 2006.

[43] I. Abt et al. (GERDA Collaboration), arXiv:0404039[hep-ex].

[44] J.D. Vergados. Phys. Rep., 133:1, 1986.

[45] S. M. Bilenky and S.P. Petcov. Rev. Mod. Phys., 59:671, 1987.

[46] S.F. King. Rep. Prog. Phys., 67:107, 2004.

[47] R.N. Mohapatra et al. Ann. Rev. Nucl. Part. Sci., 56:569, 2006.

[48] R.N. Mohapatra et al. Rep. Prog. Phys., 70:1757, 2007.

[49] H. Ejiri. Progress Particle Nuclear Physics, 64:249, 2010.

[50] R. Winter. Phys. Rev., 100:142, 1955.

[51] J.D. Vergados. Nuc. Phys. B, 218:109, 1983.

[52] J. Bernabeu, A de Rujula, and C. Jarlskog. Phys. Rev. C, 223:15, 1983.

[53] M. Doi and T. Kotani. Prog. Theor. Phys., 89:139, 1993.

[54] M.I. Krivoruchenko, F. Šimkovic, D. Frekers, and A. Faessler. Nucl. Phys. A, 859:140, 2011.

[55] S. Eliseev et al. Phys. Rev. Lett., 106:052504, 2011.

[56] K. Blaum. Phys. Rep., 425:1, 2006.

[57] H. Primakoff and S.P. Rosen. Annu. Rev. Nucl. Part. Sci, 31:145, 1981.

[58] P. Domin, S. Kovalenko, A. Faessler, and F. Šimkovic. Phys. Rev. C, 70:065501, 2004.

[59] C. Dohmen et al. (SINDRUM II Collaboration). Phys. Lett. B, 317:631, 1993.

[60] J.H. Missimer, R.N. Mohapatra, and N.C. Mukhopadhyay. Phys. Rev. D, 50:2067, 1994.

[61] F. Šimkovic, A. Faessler, S. Kovalenko, and I. Schmidt. Phys. Rev. D, 66:033005, 2002.

[62] W.C. Haxton and G.S. Stephenson Jr. Prog. Part. Nucl. Phys., 12:409, 1984.

[63] M. Doi, T. Kotani, and E. Tagasugi. Prog. Theor. Phys. (Supp.), 83:1, 1985.

[64] T. Tomoda. Rep. Prog. Phys., 54:53, 1991. 
[65] J. Suhonen and O. Civitarese. Phys. Rep., 300:123, 1998.

[66] A. Faessler and F. Šmkovic. J. Phys. G, 24:2139, 1998.

[67] J.D. Vergados. Phys. Rep., 361:1, 2002.

[68] W. Rodejohann. Int. J. Mod. Phys. E, 20:1833, 2011.

[69] Z. Sujkowski and S. Wycech. Phys. Rev. C, 70:052501, 2004.

[70] F. Šimkovic and M.I Krivoruchenko. Phys. Part. Nuc., 6:298, 2009.

[71] P. Belli et al. Eur. Phys. J. A, 47:91, 2011.

[72] J. Suhonen, P.C. Divari, L.D. Skouras, and I. D. Johnstone. Phys. Rev. C, 55:714, 1997.

[73] J. Retamosa, E. Caurier, and F. Novacki. Phys. Rev. C, 51:371, 1995.

[74] E. Caurier, F. Novacki, A. Poves, and J. Retamosa. Phys. Lett., 77:1954, 1996.

[75] J. Sinatkas, L.D. Skouras, D. Strottman, and J.D. Vergados. J. Phys. G, 18:1377, 1992.

[76] E. Caurier, A. Poves, and A.P Zucker. Phys. Lett B, 252:13, 1990.

[77] Y. Fukuda and others (SuperKamiokande Collaboration). Phys. Rev. Lett., 81:1562, 1998.

[78] B. Aharmin et al. Phys. Rev. C, 72:055502, 2005.

[79] M. Apollonio and others (CHOOZ Collaboration). Phys. Lett. B, 446:415, 1999.

[80] T. Araki et al. Phys. Rev. Lett., 94:081801, 2005.

[81] T. Schwetz, M. Tórtola, and J.W.F. Valle. New J. Phys., 10:113011, 2008.

[82] M. Duerr, M. Lindner, and A. Merle. JHEP, 1106:091, 2011.

[83] A. Cervera, A. Donini, M. B. Gavela, J. J.Gomez Cadenas, P. Hernandez, O. Mena, and S. Rigolin. Nuc. Phys. B, 579:17, 2000.

[84] S.K. Agarwalla, arXiv:1110.3681 [hep-ph].

[85] M.C. Gonzales-Garcia and M. Maltoni. Phys. Rep., 460:1, 2008.

[86] R. N. Mohapatra. Nucl. Phys. Proc. Suppl., 77:376, 1999. hep-ph/9808284.

[87] G. Pantis, F. Šimkovic, J.D. Vergados, and A. Faessler. Phys. Rev. C, 53:695, 1996.

[88] R. Mohapatra. Phys. Rev. D, 34:3457, 1986.

[89] J.D. Vergados. Phys. Lett. B, 184:55, 1987.

[90] M. Hirsch, H.V. Klapdor-Kleingrothaus, and S.G Kovalenko. Phys. Lett., 75:17, 1995.

[91] H. Päs, M. Hirsch, and H.V. Klapdor-Kleingrothaus. Phys. Lett. B, 459:450, 1999.

[92] A. Wodecki, W. Kamiński, and S. Pagerka. Phys. Lett. B, 413:342, 1997.

[93] A. Faessler, S. Kovalenko, and F. Šimkovic. Phys. Rev. D, 58:115004, 1998.

[94] A. Faessler, S. Kovalenko, and F. Šimkovic. Phys. Rev. D, 58:055004, 1998.

[95] M. Hirsch and J.W.F. Valle. Nucl. Phys. B, 557:60, 2001.

[96] J.D. Vergados. Phys. Rev. C, 24:640, 2981.

[97] B. Pontecorvo. Phys. Lett. B, 26:630, 1968.

[98] J.D. Vergados. Phys. Rev. D, 25:914, 1982.

[99] J.D. Vergados. Nucl. Phys. B, 250:618, 1985.

[100] T. Tomoda, A. Faessler, K. W. Schmid, and F. Grummer. Phys. Lett. B, 157:4, 1985.

[101] F. Šimkovic, G. Pantis, J.D. Vergados, and A. Faessler. Phys. Rev. C, 60:055502, 1999.

[102] F. Šimkovic nd G.V. Efimov, M.A. Ivanov, and V.E. Lyubovitskij. Z. Phys. A, 341:193, 1992.

[103] J.D. Vergados. Nuc. Phys. A, 506:482, 1990.

[104] G. Pantis and J.D. Vergados. Phys. Lett B, 242:1, 1990.

[105] A. Faessler, W.A. Kamiński, G. Pantis, and J.D. Vergados. Phys. Rev. C, 43:21, 1991.

[106] S.B. Khadkikar J. Suhonen and A. Faessler. Phys. Lett. B, 237:8, 1990.

[107] J.D. Vergados. Phys. Rev. C, 13:865, 1976.

[108] W. C. Haxton, G. S. Stephenson, and D. Strottman. Phys. Rev. D, 25:2360, 1982.

[109] L. Skouras and J.D. Vergados. Phys. Rev. C, 28:2122, 1983.

[110] L. Zhao, B.A. Brown, and W.A. Richter. Phys. Rev. C, 42:1120, 1990.

[111] L . Zhao and B.A. Brown. Phys. Rev. C, 47:2641, 1993.

[112] R.B. Radha et al. Phys. Rev. Lett., 76:2642, 1996.

[113] T. Sebe H. Nakada and K. Muto. Nucl. Phys. A, 607:235, 1996.

[114] D.J. Dean S.E. Koonin and K. Langanke. Phys. Rep., 278:1, 1997. 
[115] P. Vogel and M.R. Zirnbauer. Phys. Rev. Lett, 57:3148, 1986.

[116] O. Civitarese, A. Faessler, and T. Tomoda. Phys. Lett. B, 194:11, 1987.

[117] K. Muto and H.V. Klapdor. Phys. Lett. B, 208:53, 1988.

[118] J. Engel, P. Vogel, X.D. Ji, and S. Pittel. Phys. Lett. B, 225:5, 1989.

[119] A. A. Raduta, A. Faessler, S. Stoica, and W. A. Kamiński. Phys. Lett. B, 254:7, 1991.

[120] A. Griffiths and P. Vogel. Phys. Rev. C, 46:181, 1992.

[121] J. Suhonen and O. Civitarese. Phys. Lett. B, 308:212, 1993.

[122] O. Civitarese and J. Suhonen. Nuc. Phys. A, 575:251, 1994.

[123] F. Šimkovic, J., Schwieger, M. Veselský, G. Pantis, and A. Faessler. Phys. Lett. B, 393:267, 1997.

[124] F. Šimkovic, G. Pantis, and A. Faessler. Prog Part. Phys., 40:285, 1998.

[125] M.K. Cheoun, A. Bobyk, A. Faessler, F. Šimkovic, and G. Teneva. Nucl. Phys. A, 561:74, 1993.

[126] K. Muto. Phys. Lett. B, 391:243, 1997.

[127] J. Toivanen and J. Suhonen. Phys. Rev. Lett., 75:410, 1995.

[128] J. Schwieger, F. Šimkovic, and A. Faessler. Nuc. Phys. A, 600:179, 1996.

[129] P.K. Rath, R. Chandra, K. Chaturvedi, P.K. Raina, and J.G. Hirsch. Phys. Rev. C, 82:064310, 2010.

[130] J. Barea and F. Iachello. Phys. Rev. C, 79:044301, 2009.

[131] T.R. Rodrigez and G. Martinez-Pinedo. Phys. Rev. Lett., 105:252503, 2010.

[132] H. Ejiri. Phys. Rep., 338:265, 2000.

[133] A.Yu. Smirnov, arXiv: hep-ph/0411194.

[134] S. Weinberg. Phys. Rev. Lett., 43:1566, 1979.

[135] A. Abada, C.Biggio, F. Bonnet, M.B. Gavela, and T. Hambye. JHEP, 0712:061, 2007. arXiv:0707.4058 (hep-ph).

[136] M. Magg and C. Wetterich. Phys. Lett. B, 94:61, 1980.

[137] M. Magg and C. Wetterich. Nuc. Phys. B, 181:287, 1981.

[138] R. N. Mohapatra and G. Senjanovich. Phys. Rev. D, 23:165, 1981.

[139] A. Abada, C.Biggio, F. Bonnet, M.B. Gavela, and T. Hambye. Phys. Rev. D, 78:033007, 2008. arXiv:0803.0481 (hep-ph).

[140] J. Chakrabortty, A. Dighe, S. Goswami, and S. Ray. Nuc. Phys. B, 820:116, 2009. arXiv:0812.2776(hep-ph).

[141] C. Boehm, Y. Farzan, T. Hambye, S. Palomares-Ruiz, and S. Pascoli. Phys. Rev. D, 77:043516, 2008.

[142] O. Haug, J.D. Vergados, A. Faessler, and S. Kovalenko. Nucl. Phys. B, 565:38, 2000.

[143] V. Bednyakov, A. Faessler, and S. Kovalenko. Phys. Lett. B, 442:203, 1998.

[144] M. Góźdź, W.A. Kamiński, and F. Šimkovic. Phys. Rev. D, 70:095005, 2004.

[145] M. Góźdź, W.A. Kamiński, F. Šimkovic, and A. Faessler. Phys. Rev. D, 74:055007, 2006.

[146] S. Pascoli, S.T. Petcov, and A. Riotto. Nucl. Phys. B, 774:1, 2007.

[147] G. Altarelli and F. Feruglio. Rev. Mod. Phys., 82:2701, 2010.

[148] H. Ishimori, T. Kobayashi, H. Ohki, H. Okada, Y. Shimizu, and M. Tanimoto. Prog. Theor. Phys. Suppl., 183:1, 2010.

[149] I. Antoniadis, G.K. Leontaris, and J. Rizos. Phys. Lett. B, 245:161, 1990.

[150] G.K. Leontaris and J. Rizos. Nucl. Phys. B, 554:3, 1999.

[151] A.E. Faraggi, C. Kounas, and J. Rizos. Phys. Lett. B, 648:84, 2007.

[152] P. Ramond, R.G. Roberts, and G.G. Ross. Nucl. Phys. B, 406:19, 1993.

[153] L.E. Ibañez and R. Richter. JHEP, 0903:090, 2009.

[154] P. Anastasopoulos, E. Kiritsis, and A. Lionetto. JHEP, 0908:026, 2009.

[155] G.K. Leontaris. J. Mod. Phys. A, 24:6035, 2009.

[156] G.K. Leontaris and N. Vlachos. JHEP, 1001:016, 2010.

[157] R.N. Mohapatra et al, A White Paper, arXiv:hep-ph/0510213.

[158] A. Habig et al. (MINOS Collaboration). Mod. Phys. Lett. A, 25:1219, 2010.

[159] A. Gando et al. (KamLAND Collaboration). Phys. Rev. D, 83:052002, 2011. 
[160] F.P. An et al. (Daya Bay Collaboration), arXiv:1203.1669 hep-ex].

[161] K. Abe et al. (T2K Collaboration). Phys. Rev. Lett., 107:041801, 2011.

[162] H.D. Kerret (Double Chooz Collaboration), LowNu11, November 9-12, 2011, Seoul National University, Seoul, Korea.

[163] J.K. Ahn et al. (RENO Collaboration), arXiv:1204.0626 hep-ex].

[164] S. Pascoli, S.T. Petcov, and T. Schwetz. Nuc. Phys. B, 734:24, 2006.

[165] Osipowicz A et al (KATRIN Collaboration) 2001 hep-ex/0109033

Angrik $\mathrm{J}$ et al (KATRIN Collaboration) 2004 KATRIN Design Report http://bibliothek.fzk.de/zb/berichte/FZKA7090.pdf.

[166] E.W. Otten and C. Weinheimer. Rep. Prog. Phys., 71:086201, 2008.

[167] E. Andreotti (MARE Collaboration). Nucl. Instrum. Meth., 572:208, 2007.

[168] K. N. Abazajian et al. Astropart. Phys., 35:177, 2011.

[169] S. A. Thomas, F. B. Abdalla, and O. Lahav. Phys.Rev.Let., 105:031301, 2010.

[170] S.M. Bilenky, A. Faessler, W. Potzel, and F. Šimkovic. Eur. Phys. J., 71:1754, 2011.

[171] S.A. Thomas, F.B. Abdalla, and O. Lahav. Phys. Rev. Lett, 105:031301, 2010.

[172] P.D. Serpico. Phys. Rev. Lett, 98:171301, 2007.

[173] K.N. Abazajian et al. Astropart. Phys., 35:177, 2011.

[174] F. Šimkovic, A. Faessler, H. Müther, V. Rodin, and M. Stauf. Phys. Rev. C, 79:055501, 2009.

[175] S. Schoenert (GERDA Collaboration). J. Phys. Conf. Ser., 203:012014, 2010.

[176] F. Alessandria et al. (CUORE Collaboration), arXiv:1109.0494 [nucl-ex].

[177] N. Ackerman et al. (The EXO Collaboration). Phys. Rev. Lett., 107:212501, 2011.

[178] R. Gornea (EXO Collaboration). J. Phys. Conf. Ser., 259:012039, 2010.

[179] S.R. Elliott et al. MAJORANA Collaboration 2009 Private communication, 2009.

[180] R. Arnold et al. (SuperNEMO Collaboration). Eur. Phys. J, 70:927, 2010.

[181] Ch. Kraus (SNO+ Collaboration). Prog. Part. Nucl. Phys., 64:273, 2010.

[182] A.S. Barabash. Phys. Atom. Nucl., 70:1191, 2007.

[183] A.S. Barabash. AIP Conf. Proc., 1417:5, 2011.

[184] Dong-Liang Fang, A. Faessler, V. Rodin, and F. Šimkovic. Phys. Rev. C, 82:051301, 2010.

[185] G. Mention et al. Phys. Rev. D, 83:073006, 2011.

[186] M. Maltoni J. Kopp and T. Schwetz. Phys. Rev. Lett., 107:091801, 2011.

[187] J. Barry, W. Rodejohann, and He Zhang. JHEP, 1107:091, 2011.

[188] S. Pascoli, S.T. Petcov, and A. Riotto. Phys. Rev. D, 75:083511, 2007.

[189] A. Atre, T. Han, S. Pascoli, and B. Zhang. JHEP, 0905:030, 2009.

[190] G. Pantis, F. Šimkovic, J.D. Vergados, and A. Faessler. Phys. Rev. C, 53:695, 1996.

[191] A. Faessler, A. Meroni, S. T. Petcov, F. Šimkovic, and J. D. Vergados. Phys. Rev. D, 83:113003, 2011.

[192] J.D. Vergados. Phys. Rep., 361:1, 2002.

[193] O. Civitarese, M. Reboiro, and P. Vogel. Phys. Rev. C, 56:1840, 1997.

[194] J. Engel, S. Pittel, M. Stoitsov, P. Vogel, and J. Dukelsky. Phys. Rev. C, 55:1781, 1997.

[195] G. Martinez-Pinedo, K. Langanke, and P. Vogel. Nucl. Phys. A, 651:379, 1999.

[196] R.N. Mohapatra, A. Perez-Lorenzana, and C.A. de S. Pires. Phys. Lett. B, 491:143, 2000.

[197] R. Tomas, H. Päs, and J.W.F. Valle. Phys. Rev. D, 64:095005, 2001.

[198] J.C. Montero, C.A. de S. Pires, and V. Pleitez. Phys. Rev. D, 64:096001, 2001.

[199] K.S. Babu and R.N. Mohapatra. Phys. Rev. Lett., 75:2276, 1995.

[200] M. Hirsch, H.V. Klapdor-Kleingrothaus, and S.G Kovalenko. Phys. Rev. D, 53:1329, 1996.

[201] H. Päs, M. Hirsch, H.V. Klapdor-Kleingrothaus, and S.G. Kovalenko. Phys. Lett. B, 453:194, 1999.

[202] A. Faessler, Th. Gutsche, S. Kovalenko, and F. Šimkovic. Phys. Rev. D, 77:113012, 2008.

[203] J.D. Vergados, A. Faessler, and H. Toki. Phys. Rev. D, 81:034018, 2010.

[204] S.L. Adler et al. Phys. Rev. D, 11:3309, 1975.

[205] S.R. Elliott and P. Vogel. Annu. Rev. Nucl. Part. Sci., 52:115, 2002. 
[206] E. Der Mateosian and M. Goldharber. Phys. Rev., 146:810, 1966.

[207] E. Fiorini, A. Pullia, G. Bertolini, F. Cappellani, and G. Restelli. Phys. Lett B., 25:602, 1967.

[208] E. Fiorini et al. Nuovo Cimento A, 13:747, 1973.

[209] M. H. Schapiro, S. Frankel, S. Koicki, W. D. Wales, and G. T. Wood. Phys. Rev., 154:1050, 1967.

[210] R. K. Bardin, P. G. Gollon, J. D. Ullman, and C. S. Wu. Phys. Lett. B, 26:112, 1967.

[211] R. K. Bardin, P. G. Gollon, J. D. Ullman, and C. S. Wu. Nuc. Phys. A, 158:337, 1970.

[212] B. T. Cleveland et al. Phys. Rev. Lett., 35:757, 1975.

[213] E. Bellotti, E. Fiorini, C. Liguori, A. Pallia, A. Sarracino, and L. Zanotti. Lett. Nuovo Cimento, 33:273, 1982.

[214] F. T. Avignone et al. Phys. Rev. Lett., 50:721, 1983.

[215] H. Ejiri et al. Proc. Neutrino mass and low energy weak interactions, (Teremark), 1984 (World Scientific) p. 383.

[216] E. Ejiri et al. Nucl. Phys. A, 448:271, 1986.

[217] E. Ejiri et al. J. Phys. G; Nucl.Phys., 13:839, 1987.

[218] T. Kirsten and H. W. Muller. Earth and Planetary Sci. Lett., 6:271, 1969.

[219] T. Kirsten and H. W. Muller. Earth and Planetary Sci. Lett., 7:300, 1969.

[220] H. Ejiri et al. Phys. Lett. B, 258:17, 1991.

[221] H. Ejiri et al. J. Phys. G, 17:S155, 1991.

[222] E. Fiorini and T. O. Niinikoski. Nucl. Instr. Methods, 224:83, 1984.

[223] A. Alessandrello et al. Nucl. Phys. B (Proc. Suppl.), 28:233, 1992.

[224] A. Alessandrello et al. Phys. Lett. B, 420:109, 1998.

[225] L. Gironi et al. Nucl. Instr. Methods A, 617:478, 2010.

[226] T. Bernatowicz et al. Phys. Rev. C, 47:8066, 1993.

[227] H. V. Klapdor-Kleingrothaus et al. Phys. Rev. D, 63:073005, 2001.

[228] H. V. Klapdor-Kleingrothaus et al. Phys. Lett. A, 16:2469, 2001.

[229] C. E. Aalsethand others. Phys. Rev. C, 59:2108, 1999.

[230] C. E. Aalsethand others. Phys. Rev. D, 65:092007, 2002.

[231] C. Arnaboldi et al. Phys. Rev. C, 78:035502, 2008.

[232] C. Arnaboldi et al. Astropart. Phys., 34:822, 2011.

[233] E. Ejiri et al. Phys. Rev. C, 63:065501, 2001.

[234] C. Arnaboldi et al. JETP Lett., 80:377, 2004.

[235] C. Arnaboldi et al. Phys. Rev. Lett., 95:142501, 2005.

[236] J. Menéndez, A. Poves, E. Caurier, and F. Nowacki. Nucl. Phys. A, 818:139, 2009.

[237] I. Ogawa et al. Nucl. Phys. A, 730:215, 2004.

[238] F.A. Danevich et al. Phys. Rev. C, 68:035501, 2003.

[239] O. Cremonesi and The CUORE collaboration, Talk given at MEDEX11 conf., Prague, 2011, http://medex11.utef.cvut.cz/.

[240] R. L. Flack, NEMOIII collaboration 2008 Proc. Neutrino08 (Christchurch), 2008.

[241] A.S. Barabash. Phys. Rev. C, 81:035501, 2010.

[242] E. Aguayo et al. (The MAJORANA collaboration). AIP Conf. Proc., 1417:95, 2011.

[243] I. Ogawa I et al. Private communication, 2009.

[244] H. Ejiri et al. Phys. Rev. Lett., 85:2917, 2000.

[245] H. Ejiri. Mod. Phys. Lett. A, 22:1277, 2007.

[246] H. Nakamura et al. J. Phys. Soc. Japan, 76:114201, 2007.

[247] H. Ejiri et al. Eur. Phys. Jour., 162:239, 2008.

[248] V. M. Gehman, P.J. Doe, R. G. H. Robertson, D. J. Will, H. Ejiri, and R. Hazama. Nucl. Instr. Method Phys. Research A, 622:602, 2010.

[249] H. Ejiri, J. Engel, and N. Kudomi. Phys. Lett. B, 530:27, 2002.

[250] A. Chapon and The SuperNEMO collaboration. AIP Conf. Proc., 1417:18, 2011.

[251] S.K. Kim, AMoRE Collaboration, Talk given at MEDEX11 conf., Prague, 2011, 
http://medex11.utef.cvut.cz/

[252] K. Zuber and The COBRA collaboration, Talk given at MEDEX11 conf., Prague, 2011, http://medex11.utef.cvut.cz/

[253] G. Gratta, et al., Talk given at Neutrino 08 conf., New Zealand, 2008, http://www2.phys.canterbury.ac.nz/ jaa53/.

[254] M. Auger et al, EXO Collaboration 2012, arXiv:1205.5608v1 [hep-ex].

[255] N. Ackermann et al. Phys. Rev. Lett., 107:212501, 2011.

[256] Y. Efremenko and The KamLAND-Zen Collaboration, Talk given at MEDEX11 conf., Prague, 2011, http://medex11.utef.cvut.cz/

[257] V. Álvarez et al (NEXT collaboration), arXiv:1202.0721v2 [physics.ins-det].

[258] R. Raghavan, in Proceedings of NDM09, Madison 2009.

[259] N. Ishihara et al. Private communication, 2009.

[260] K. Zuber and The COBRA collaboration, Talk given at MEDEX11 conf., Prague, 2011, http://medex11.utef.cvut.cz/.

[261] H. Ejiri. Phys. Rep., 338:265, 2000. and refs. therein.

[262] H. Ejiri. Czech. J. Phys., 56:459, 2006.

[263] H. Ejiri and H. Toki. J. Phys. Soc. Japan, 65:7, 1996.

[264] J. Suhonen and M. Kortelainen. Czech J. Phys., 56:519, 2006.

[265] F. Avignone Workshop Neutr. Nucl. Phys. Stopped $\pi \mu$ Facility (Oak Ridge), 2000.

[266] H. Ejiri. Nucl. Instr. Meth. Phys. Research, 503:276, 2003.

[267] H. Ejiri et al. Phys. Rev. Lett., 21:373, 1968.

[268] H. Ejiri et al. Nucl. Phys. A, 128:388, 1969.

[269] H. Ejiri and J. Bondorf. Phys. Lett. B, 28:304, 1968.

[270] H. Akimune et al. Phys. Lett. B, 394:23, 1997.

[271] H. Akimune et al. Phys. Lett. B, 665:424, 2008.

[272] H. Dohman et al. Phys. Rev. C, 78:041602R, 2008.

[273] R.G.T. Zegers et al. Phys. Rev. Lett., 99:202501, 2007.

[274] S. Rakers et al. Phys. Rev. C, 70:054302, 2004.

[275] S. Rakers et al. Phys. Rev. C, 71:054313, 2005.

[276] E.W. Grewe et al. Phys. Rev. C, 76:054307, 2007.

[277] E.W. Grewe et al. Phys. Rev. C, 78:044301, 2008.

[278] K. Yako et al. Phys. Rev. Lett., 103:012503, 2009.

[279] D. Frekers. Prog. Part. Nucl. Phys., 64:281, 2010.

[280] P. Puppe et al. Phys. Rev. C, 84:051305, 2011.

[281] C.J. Guess et al. Phys. Rev. C, 83:064318, 2011.

[282] J. P. Schiffer et al. Phys. Rev. Lett., 100:112501, 2008.

[283] J. Abad, A. Morales, R. Nunez-Lagos, and A. F. Pacheco. Nuovo Cim. A, 75:173, 1983.

[284] H. Ejiri. J. Phys. Soc. Japan, 78:074201, 2009.

[285] H. Ejiri. J. Phys. Soc. Japan, 81:033201, 2012.

[286] A.D. Dolgov and A.Yu. Smirnov. Phys. Lett. B, 621:1, 2005.

[287] A.S. Barabash, A.D. Dolgov, F. Šimkovic R. Dvornický, and A.Yu. Smirnov. Nucl. Phys. B, 783:90, 2007.

[288] F. Šimkovic, P. Domin, and S.V. Semenov. J. Phys. G, 27:2233, 2001.

[289] P. Domin, S. Kovalenko, F. Šmkovic, and S.V. Semenov. Nucl. Phys. A, 753:337, 2005.

[290] A. Smolnikov and P. Grabmayr. Phys. Rev. C, 81:028502, 2010.

[291] V.A. Rodin, A. Faessler, F. Šimkovic, and P. Vogel. Nucl. Phys. A, 766:107, 2006.

[292] V.A. Rodin, A. Faessler, F. Šimkovic, and P. Vogel. Nucl. Phys. A, 793:213, 2007.

[293] F. Šimkovic, A. Faessler, V.A. Rodin, P. Vogel, and J. Engel. Phys. Rev. C, 77:045503, 2008.

[294] A. Poves, E. Caurier, and F. Nowacki. Eur. Phys. J. A, 36:195, 2008.

[295] E. Caurier, J. Menendez, F. Nowacki, and A. Poves. Phys. Rev.Lett., 100:052503, 2008.

[296] M. Horoi, S. Stoica, and B. A. Brown. Phys. Rev. C, 75:034303, 2007. 
[297] M. Horoi and S. Stoica. Phys. Rev. C, 81:024321, 2010.

[298] F. Šimkovic, A. Faessler, and P. Vogel. Phys. Rev. C, 79:015502, 2009.

[299] G.A. Miller and J. E. Spencer. Ann. Phys. (NY), 100:562, 1976.

[300] M. Kortelainen and J. Suhonen. Phys. Rev. C, 75:051303(R), 2007.

[301] M. Kortelainen and J. Suhonen. Phys. Rev. C, 76:024315, 2007.

[302] J. Suhonen. Nucl. Phys. A, 864:63, 2011.

[303] V.A. Rodin, A. Faessler, F. Šimkovic, and P. Vogel. Phys. Rev. C, 68:044302, 2003.

[304] J. Suhonen. Phys. Lett. B, 607:87, 2005.

[305] O. Civitarese and J. Suhonen. Nucl. Phys. A, 761:313, 2005.

[306] D.S. Delion, J. Dukelsky, and P. Schuck. Phys. Rev. C, 55:2340, 1997.

[307] F. Krmpotić et al. Nucl. Phys. A, 637:295, 1998.

[308] K. Muto. Nucl. Phys. A, 577:415c, 1994.

[309] J. Engel and G. Hagen. Phys. Rev. C, 79:064317, 2009.

[310] M. Kortelainen, O. Civitarese, J. Suhonen, and J. Toivanen. Phys. Lett. B, 647:128, 2007.

[311] R. Roth, H. Hergert, P. Papakonstantinou, T. Neff, and H. Feldmeier. Phys. Rev. C, 72:034002, 2005.

[312] J. Engel, J. Carlson, and R.B. Wiringa. Phys. Rev. C, 83:034317, 2011.

[313] F. Šimkovic, L. Pacearescu, and A. Faessler. Nucl. Phys. A, 733:321, 2004.

[314] R. Alvarez-Rodriguez, P. Sarriguren, E. Moya de Guerra, L. Pacearescu, A. Faessler, and F. Šimkovic. Phys. Rev. C, 70:064309, 2004.

[315] R. Chandra, J. Singh, P.K. Rath, P.K. Raina, and J. G. Hirsch. Eur. Phys. J. A, 23:223, 2005.

[316] R. Chandra K. Chaturvedi, P.K. Rath, P.K. Raina, and J.G. Hirsch. Phys. Rev. C, 78:054302, 2008.

[317] M. Yousuf Saleh, V. Rodin, A. Faessler, and F. Šimkovic. Phys. Rev. C, 79:014314, 2009.

[318] Dong-Liang Fang, A. Faessler, V. Rodin, Mohamed Saleh, and F. Šimkovic. Phys. Rev. C, 81:037303, 2010.

[319] V. Rodin, A. Faessler, and F. Šimkovic. Phys. Rev. C, 83:034320, 2011.

[320] J.P. Schiffer et al. Phys. Rev. Lett., 100:112501, 2008.

[321] B.P. Kay et al. Phys. Rev. C, 79:021301, 2009.

[322] J. Menéndez, A. Poves, E. Caurier, and F. Nowacki. Phys. Rev. C, 80:048501, 2009.

[323] J. Suhonen and O. Civitarese. Phys. Lett. B, 668:277, 2008.

[324] J. Suhonen. Nucl. Phys. A, 853:36, 2011.

[325] A. Faessler, G.L. Fogli, V. Rodin E. Lisi, A.M. Rotunno, and F. Šimkovic. J. Phys. G, 35:075104, 2008.

[326] A. Faessler, G.L. Fogli, E. Lisi, V. Rodin, and F. Šimkovic. Phys. Rev. D, 79:053001, 2009.

[327] F. Šimkovic, R. Hodák, A. Faessler, and P. Vogel. Phys. Rev. C, 83:015502, 2011.

[328] V. Rodin and A. Faessler. Phys. Rev. C, 80:041302, 2009.

[329] P. Beneš, A. Faessler, S. Kovalenko, and F. Šimkovic. Phys. Rev. D, 71:077901, 2005.

[330] M. Mitra, G. Senjanović,, and F. Vissani. Nucl. Phys. B, 856:26, 2012.

[331] A. Faessler, G.L. Fogli, E. Lisi, A.M. Rotunno, and F. Šimkovic. Phys. Rev. D, 83:113015, 2011.

[332] E. Molinaro A. Ibarra and S.T. Petcov. Phys. Rev. D, 84:013005, 2011.

[333] V. Tello, M. Nemevšek, F. Nesti, G. Senjanović, and F. Vissani. Phys. Rev. Lett., 106:151801, 2011.

[334] B.C. Allanach, C.H. Kolm, and H. Päs. JHEP, 0910:026, 2009.

[335] B.C. Allanach, C.H. Kolm, and H. Päs. Phys. Rev. Let., 103:091801, 2009.

[336] V. Cirigliano, A. Kurylov, M.J. Ramsey-Musolf, and P. Vogel. Phys. Rev. Lett., 93:231802, 2004.

[337] R.N. Mohapatra and J.C. Pati. Phys. Rev. D, 11:566, 1975.

[338] J. Argyriades et al. (SuperNEMO Collaboration). Nucl. Instrum. Meth. A, 622:120, 2010.

[339] H.V. Klapdor-Kleingrothaus, I.V. Krivosheina, and I.V. Titkova. Phys. Rev. D, 73:013010, 2006.

[340] H.V. Klapdor-Kleingrothaus, I.V. Krivosheina, and I.V. Titkova. Int. J. Mod. Phys. A, 21:1159, 2006. 
[341] H.V. Klapdor-Kleingrothaus, I.V. Krivosheina, V. Mironov, and I.V. Titkova. Phys. Lett. B, 636:235, 2006.

[342] H.V. Klapdor-Kleingrothaus, I.V. Krivosheina, and I.V. Titkova. Mod. Phys. Lett. A, 21:1257, 2006.

[343] F. Deppisch, C. Jackson, I. Nasteva, and S. Söldner-Rembold. Prog. Part. Nucl. Phys., 64:278, 2010.

[344] M. Duerr, M. Lindner, and K. Zuber. Phys. Rev. D, 84:093004, 2011.

[345] H. Ejiri (MOON Collaboration). Nucl. Phys. A, 844:10c, 2010.

[346] K. Zuber. Prog. Part. Nucl. Phys., 64:267, 2010.

[347] M. Hirsch H. Päs, H.V. Klapdor-Kleingrothaus, and S.G. Kovalenko. Phys. Lett. B, 498:35, 2001.

[348] G. Prézeau, M. Ramsey-Musolf, and P. Vogel. Phys. Rev. D, 68:034016, 2003.

[349] A.V. Borisov A. Ali and D.V. Zhuridov. Phys. Rev. D, 76:093009, 2007.

[350] A.V. Borisov A. Ali and D.V. Zhuridov. Phys. Atom. Nucl., 73:2083, 2010.

[351] A. Faessler, S. Kovalenko, F. Šimkovic, and J. Schwieger. Phys. Lett. B, 78:183, 1997.

[352] F. Šimkovic and A. Faessler. Prog. Part. Nucl. Phys., 48:201, 2002.

[353] S.M. Bilenky and J.A. Grifols. Phys. Lett. B, 550:154, 2002.

[354] F. Deppisch and H. Päs. Phys. Rev. Lett., 98:232501, 2007.

[355] V.M. Gehman and S. R. Elliott. J. Phys. G, 34:667, 2007.

[356] F. Šimkovic, M. Nowak, W.A. Kamiński, A.A. Raduta, and A. Faessler. Phys. Rev. C, 64:035501, 2001.

[357] T. Tomoda. Phys. Lett. B, 474:245, 2000.

[358] M. Hirsch, K. Muto, T. Oda, and H.V. Klapdor-Kleingrothaus. Z. Phys. A, 347:151, 1994.

[359] J. Suhonen. Phys. Lett. B, 477:99, 2000.

[360] J. Suhonen. Phys. Rev. C, 62:042501, 2000.

[361] T. Tomoda, A. Faessler, K.W. Schmid, and F. Grümmer. Nucl. Phys. A, 452:591, 1986.

[362] K. Muto, E. Bender, and H.V. Klapdor. Z. Phys. A, 334:187, 1989.

[363] J. Suhonen, S.B. Khadkikar, and A. Faessler. Nucl. Phys. A, 535:509, 1991.

[364] P.K. Rath, R. Chandra, P.K. Raina, and K. Chaturvedi. Phys. Rev. C, 85:014308, 2012.

[365] F. Šimkovic, J.D. Vergados, and A. Faessler. Phys. Rev. D, 82:113015, 2010.

[366] J. Jochum (GERDA Collaboration). Prog. Part. Nucl. Phys., 64:261, 2010.

[367] S. Schonert (GERDA Collaboration). J. Phys. Conf. Ser., 203:012014, 2010.

[368] J.J. Gomez-Cadenas, J. Martin-Albo, M. Mezzetto, F. Monrabal, and M. Sorel. Riv. Nuovo Cim., 35:29, 2012.

[369] L. Lukaszuk, Z. Sujkowski, and S. Wycech. Eur. Phys. J. A, 27:63, 2006.

[370] G. Audi et al. Nucl. Phys. A, 729:3, 2003.

[371] G. Douysset et al. Phys. Rev. Lett., 86:4259, 2001.

[372] K. Blaum, Yu.N. Novikov, and G. Werth. Contemp. Phys., 51:149, 2010.

[373] M. Redshaw et al. Phys. Rev. Lett., 98:053003, 2007.

[374] M. Redshaw et al. Phys. Rev. Lett., 102:212502, 2009.

[375] N.D. Scielzo et al. Phys. Rev. C, 80:0225501, 2009.

[376] S. Rahaman et al. Phys. Rev. Lett., 103:042501, 2009.

[377] V.S. Kolhinen et al. Phys. Lett. B, 684:17, 2010.

[378] B.J. Mount, M. Redshaw, , and E. G. Myers. Phys. Rev. C, 81:032501, 2010.

[379] S. Eliseev et al. Phys. Rev. C, 83:038501, 2011.

[380] S. Eliseev et al. Phys. Rev. C, 84:012501, 2011.

[381] M. Goncharov et al. Phys. Rev. C, 84:028501, 2011.

[382] S. Eliseev et al. Phys. Rev. Lett., 107:152501, 2011.

[383] C. Droese et al. Nucl. Phys. A, 875:1, 2012.

[384] J.D. Vergados. Phys. Rev. C, 84:044328, 2011.

[385] F.B. Larkins. At. Data Nucl. Data Tables, 20:311, 1977.

[386] J.L. Campbell and T. Papp. At. Data Nucl. Data Tables, 77:1, 2001. 
[387] F. Šimkovic, M.I. Krivoruchenko, and A. Faessler. Prog. Part. Nucl. Phys., 66:446, 2011.

[388] Dong-Liang Fang, K. Blaum, S. Eliseev, A. Faessler, M.I. Krivoruchenko, V. Rodin, and F. Šimkovic. Phys. Rev. C, 85:035503, 2012.

[389] J. Suhonen. Phys. Lett. B, 701:490, 2011.

[390] A.S. Barabash, Ph. Hubert, A. Nachab, and V. Umatov. Nucl. Phys. A, 785:371, 2007.

[391] A.S. Barabash, Ph. Hubert, A. Nachab, S.I. Konovalov, I.A. Vanyushin, and V.I. Umatov. Nucl. Phys. A, 807:269, 2008.

[392] P. Belli et al. Nucl. Phys. A, 824:101, 2009.

[393] N.I. Rukhadze et al. (TGV Collaboration). Nucl. Phys. A, 852:197, 2011.

[394] D. Frekers, P. Puppe, J.H. Thies, P. Povinec, F. Šimkovic, J. Staniček, and I. Sýkora. Nucl. Phys. A, 860:1, 2011.

[395] P. Belli et al. Nucl. Phys. A, 859:126, 2011.

[396] D. Frekers, arXiv:hep-ex/0506002. 